\title{
Laser Cooling and Trapping of Atoms
}

\section{A thesis submitted for the degree of Doctor of Philosophy}

Christopher G. Townsend

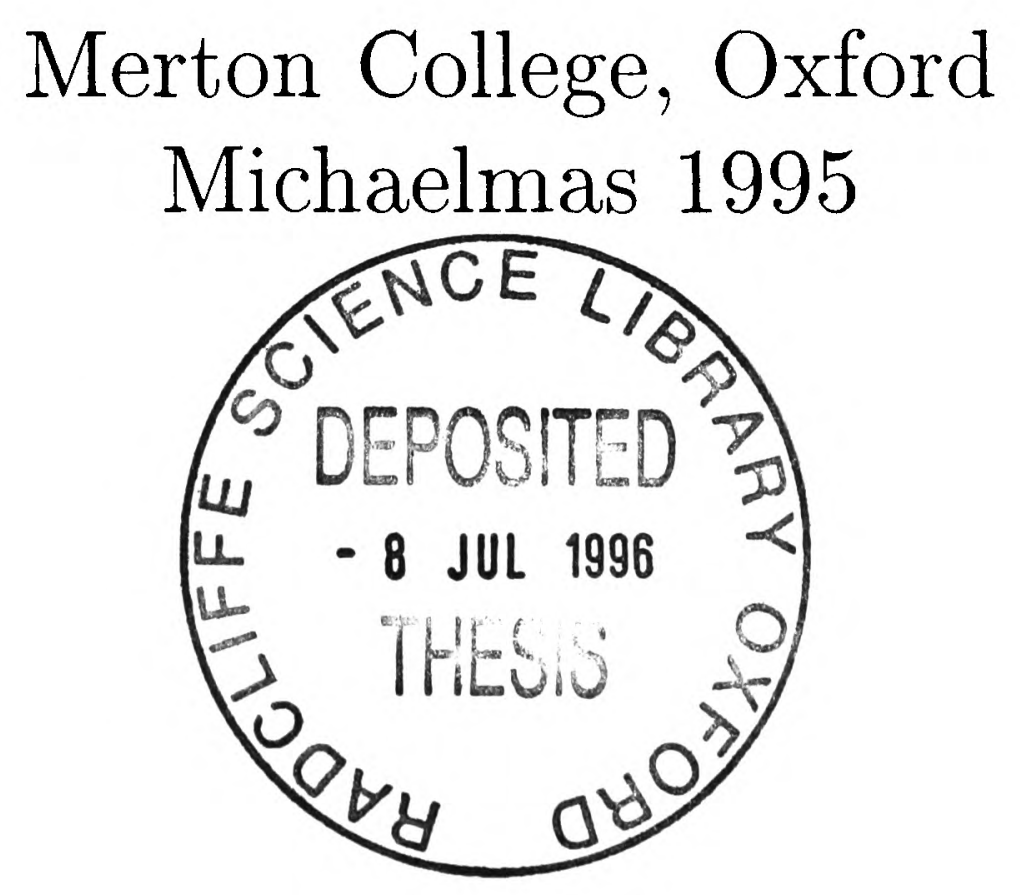




\title{
Laser Cooling and Trapping of Atoms
}

\author{
A thesis submitted for the degree of Doctor of Philosophy
}

\author{
Christopher G. Townsend, Merton College, Oxford \\ Michaelmas 1995
}

A detailed experimental and theoretical investigation of a magneto-optical trap for caesium atoms is presented. Particular emphasis has been placed on achieving high spatial number densities and low temperatures. Optimizing both of these together enables efficient evaporative cooling from a conservative trap, a procedure which has recently led to the first observations of Bose-Einstein condensation in a dilute atomic vapour.

The behaviour of a magneto-optical trap is nominally determined by four independent parameters: the detuning and intensity of the light field, the magnetic field gradient and the number of trapped atoms. A model is presented which incorporates previous treatments into a single description of the trap that encompasses a wide range of its behaviour. This model was tested quantitatively by measuring the temperature of the cloud and its spatial distribution as a function of the four parameters. The maximum density was found to be limited both by the reabsorption of photons scattered within the cloud and by a reduction of the confining force at small light shifts. The nonlinear variation with position of the restoring force was found to be significant in limiting the number of atoms confined to a high density. A maximum density in phase space (defined as the number of atoms in a box with sides of dimension one thermal de Broglie wavelength $)$ of $(1.5 \pm 0.5) \times 10^{-5}$ was observed, with a spatial density of $1.5 \times 10^{11}$ atoms per $\mathrm{cm}^{3}$.

Cold collision losses from a caesium magneto-optical trap have been studied with the purpose of assessing their influence on spatial densities. In contrast to previous measurements of similar quantities, these measurements did not require the use of an ultra-low $\left(<10^{-10}\right.$ Torr $)$ background vapour pressure. The dependence of the cold collision loss coefficient $\beta$ on the trapping intensity was measured to permit identification of the different cold collision processes. The largest loss rates observed were those due to hyperfine structure-changing collisions, with a coefficient $\beta=(2 \pm 1) \times 10^{-10} \mathrm{~cm}^{3} \mathrm{~s}^{-1}$.

A study is presented of a modified magneto-optical trap in which a fraction of the population is shelved into a hyperfine level that does not interact with the trapping light. In this so-called "dark" magneto-optical trap, improved densities of nearly $10^{12} \mathrm{~cm}^{-3}$ have been previously reported for sodium. The application of the technique to caesium is not straightforward due to the larger excited state hyperfine splittings. A simple theory for caesium is presented and its main predictions verified by measurements of density, number and temperature. A density of nearly $10^{12} \mathrm{~cm}^{-3}$ was indeed obtained but at a temperature substantially higher than in the conventional magneto-optical trap. 


\section{Acknowledgements}

There are many people without whose help this thesis would not have been possible. First and foremost I would like to extend many thanks to my supervisor Dr. Christopher Foot. I am very grateful for all the time, support and encouragement he has given me, far in excess of that normally expected from a supervisor. The many invaluable discussions with him and suggestions from him, both experimental and theoretical, have contributed enormously to this thesis and have been much appreciated. I would also like to take this opportunity to thank Dr. Derek Stacey for his valuable advice and enthusiastic encouragement. Thanks are due also to Dr. Keith Burnett for expert comments on cold collisions.

The research has involved much teamwork. Special thanks must go to Nick Edwards and Ken Zetie for endless help with the experiments and technical assistance, especially with the image acquisition system. In particular I am much indebted to Ken for having scrupulously read this thesis. I would like to thank Cath Cooper and Jürgen Rink for having patiently taught me the laser cooling ropes, and Gerd Hillenbrand for many useful and interesting theoretical discussions. Particular thanks go to Andrew Steane for collaboration and much assistance in writing the phase-space density paper.

Thanks are also due to other Clarendon friends for having helped to make the work here great fun: Andrew Simpson, Keith Turner, Dave Lucas, Bruce Warrington, Giovanna Morigi, Chiara Macchiavello, Huang Wu, Dave Meacher, Jos Martin, Pete Bance, Andrew Wilson and many others.

Finally I would like to thank my parents, my sisters Carolyn and Kate, and my girlfriend Sophie for constant support and encouragement throughout these three years. 


\section{Contents}

1 Introduction $\quad 1$

1.1 The magneto-optical trap .................. 3

1.1.1 Motion-induced orientation .............. 3

1.1 .2 The Doppler MOT . . . . . . . . . . . . . . . . 6

1.1.3 The sub-Doppler MOT ............... 8

2 Atom-atom interactions in the MOT 10

2.1 Introduction . . . . . . . . . . . . . . 10

2.2 The static MOT . . . . . . . . . . . . . . 11

2.2 .1 Temperature-limited regime ........................ 14

2.2 .2 Multiple scattering regime . . . . . . . . . . . . 17

2.2 .3 Two-component regime . . . . . . . . . . . . . . 21

2.2 .4 Regime boundaries ............................ 24

2.2 .5 Optically thick regime . . . . . . . . . . . 26

2.2 .6 Summary of the static MOT . . . . . . . . . . . 27

2.2 .7 Limitations of the model . . . . . . . . . . . . . . . 27

2.3 The time-dependent MOT . . . . . . . . . . . . . . . 28

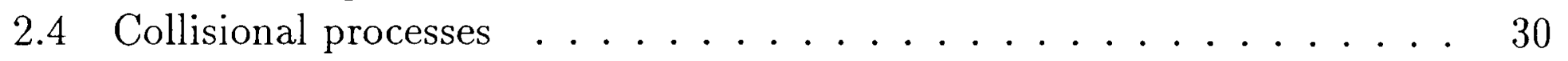

2.4.1 Collisions with the background vapour ......... 31

2.4 .2 Cold collisions . . . . . . . . . . . . . . . 34

2.4.2.1 Introduction . . . . . . . . . . . . 34

2.4.2.2 Groundstate collisions ............. 36

2.4.2.2.1 Elastic ground state processes. . . . . . . 38

2.4.2.2.2 Inelastic ground state processes. . . . . . . . 38

2.4.2.3 Light-assisted collisions . . . . . . . . . 39

3 Experimental Setup $\quad 42$

3.1 Introduction . . . . . . . . . . . . . . . . . . . . 42

3.2 The diode lasers . . . . . . . . . . . . . . . . . . . . . . . . . . . . . . 42

3.2 .1 General .............................. 42

3.2.2 Spectral characteristics of the slave laser . . . . . . . . . . . 43

3.2.3 Frequency stabilization . . . . . . . . . . . . . 44

3.3 The optical setup . . . . . . . . . . . . . . . . . . . 46

3.3 .1 General features . . . . . . . . . . . . . . . . . 46

3.3.2 Details of the optical and laser systems . . . . . . . . . 46 
3.3.3 Frequency and intensity control . . . . . . . . . . . . . 49

3.3.4 Trapping beam quality . . . . . . . . . . . . 51

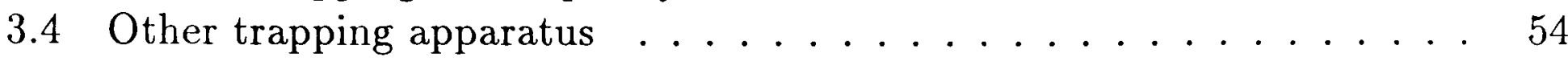

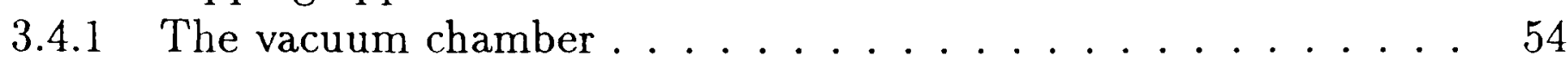

3.4 .2 The caesium source . . . . . . . . . . . . . . . . . . 54

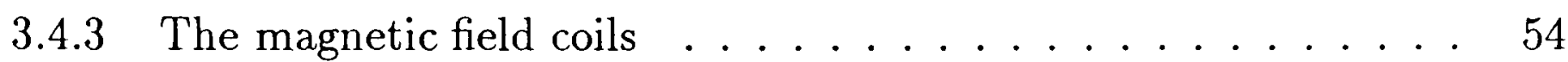

3.4 .4 Nulling coils . . . . . . . . . . . . . . . 55

3.5 Measuring techniques and data acquisition . . . . . . . . 55

3.5.1 Fluorescence detection ................ . . . . . . . . . . . 55

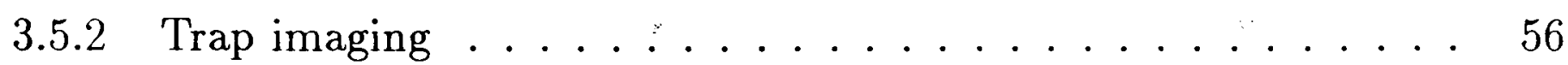

3.5 .3 Image acquisition . . . . . . . . . . . . . . . 58

3.5.4 Measurement of temperature . . . . . . . . . . . 59

3.6 Computer control . . . . . . . . . . . . . . . . 62

4 Phase-space density in the MOT 63

4.1 Introduction . . . . . . . . . . . . . . . . 63

4.2 Experimental arrangements .................. 64

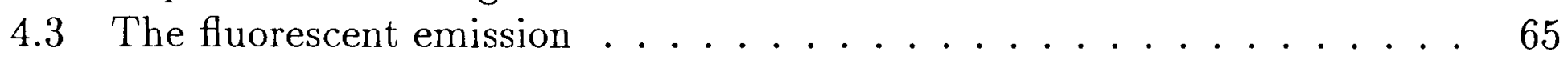

4.4 Temperature measurements . . . . . . . . . . . . . . . . . . . . 69

4.5 Measurement of $\kappa \ldots \ldots \ldots \ldots \ldots$. . . . . . . . . . . . . . . . . . . . . . . . . .

4.6 Nonlinearity of the confining force . . . . . . . . . . . . . . . 76

4.7 The position damping time . . . . . . . . . . . . . . 82

4.8 Density measurements . . . . . . . . . . . . . . . 84

4.8 .1 Introduction . . . . . . . . . . . . . . . . . 84

4.8.2 The measuring technique ..................... 86

4.8.3 The transient technique .......................... 89

4.8.4 The density behaviour . . . . . . . . . . . . . 91

4.8.5 Comparison with previous work . . . . . . . . . . 99 96

4.9 Phase-space density . . . . . . . . . . . . . . . . . . . . . 96

4.10 Concluding summary . . . . . . . . . . . . . . . . 99

5 Measurement of cold collision losses 101

5.1 Introduction . . . . . . . . . . . . . . . . . 101

5.2 General technique . . . . . . . . . . . . . . . . . 102

5.3 Confirming the interpretation of cold collisions . . . . . . . . . 103

5.4 Discussion of method . . . . . . . . . . . . . . 106

5.5 Measurements of $\beta \ldots \ldots \ldots \ldots$

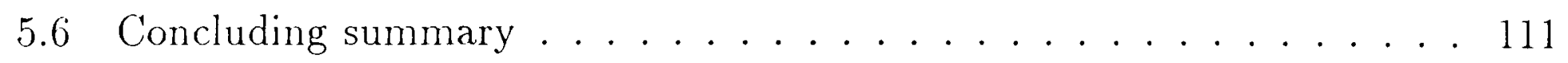

6 The caesium dark MOT 112

6.1 Introduction . . . . . . . . . . . . . . . . 112

6.2 The dark MOT . . . . . . . . . . . . . . . 113

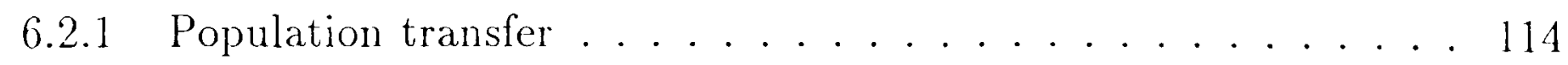

6.2 .2 Dark MOT density . . . . . . . . . . . . . . 117

6.2 .3 Temperature and phase-space density . . . . . . . . 121 
6.2.4 Trapped numbers and lifetimes ............. . . 122

6.2.5 Monte-Carlo simulation of a dark MOT . . . . . . . . . . . 123

6.3 Experimental setup . . . . . . . . . . . . . . . . 126

6.3.1 The temporal dark MOT . . . . . . . . . . 126

6.3 .2 The spatial dark MOT . . . . . . . . . . . 126

6.4 Dark MOT experiments . . . . . . . . . . . . . 128

6.4.1 Effect of depumping on trap temperature . . . . . . . . . 128

6.4 .2 Saturation of repumping . . . . . . . . . . . . . 129

6.4.3 Number and cross-section measurements . . . . . . . . . . . . 131

6.4 .4 Density measurements . . . . . . . . . . . . . . . 132

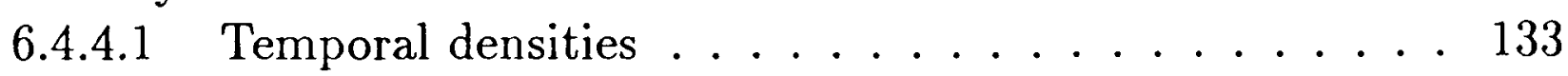

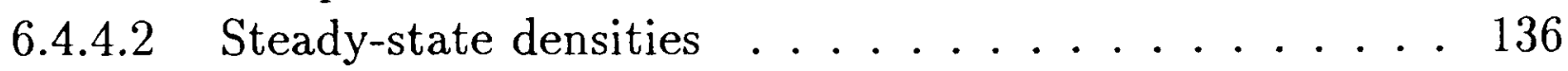

6.5 Concluding summary . . . . . . . . . . . . . . 139

7 Concluding remarks $\quad 141$

$\begin{array}{lr}\text { Bibliography } & 143\end{array}$ 


\section{Chapter 1}

\section{Introduction}

Much progress has been made in the effort to optically cool and confine large samples of atomic vapour since the initial proposal in 1975 to damp the velocities of atoms with laser light $[37,91]$. The motivation behind the research has been both to develop sources of cold atoms for use in fundamental experiments and to understand the basic processes important at temperatures below $1 \mathrm{mK}$. Atoms routinely cooled in laser fields to temperatures of a few microkelvin have been used for precision measurements, atomic interferometry and atomic clocks, whilst the high densities that have been attained have revealed a wealth of novel collision physics, not apparent at higher temperatures. Recently laser-cooled atoms have been magnetically trapped and evaporatively cooled, and this has led to the first observations of Bose-Einstein condensation (BEC) in a dilute atomic vapour $[1,7,22]$.

The magneto-optical trap (MOT) has been a crucial element in most of the above types of experiment. Its scheme was proposed by Dalibard in 1987 [18] and realized experimentally by Raab et al. [71] in the same year. A simple configuration of laser beams and a quadrupole magnetic field is used both to slow and confine large samples of neutral atoms, and densities in excess of $10^{10}$ atoms per $\mathrm{cm}^{3}$ at temperatures of 10 microkelvin are readily attainable. The MOT is robust and convenient and for the heavy alkalis can be formed in a low pressure vapour cell illuminated by diode lasers, making it also cheap and compact.

Despite its widespread use the basic behaviour of the MOT is still far from being completely understood. In normal operation the optical thickness of the cloud is significant and dramatic behaviour is induced by collective optical interactions. Unusual collision effects become dominant at low temperatures and in the presence of a near-resonant light field and these can also influence the behaviour. In addition the polarization and amplitude of a laser field in three dimensions is usually not well known. Thus generally the MOT is a complicated system of cooling and trapping forces radically modified by atom-atom interactions, and a complete theoretical model is difficult to construct. To build a picture one relies on simple theories quantified by experimental measurement, and part of this thesis has been aimed at using this approach to improve the understanding of the MOT.

To attain the conditions necessary to observe collective quantum effects such as $\mathrm{BEC}$ one is interested in obtaining a low temperature in combination with a high 
spatial density. For an atom of mass $m$ the "thermal" de Broglie wavelength $\Lambda$ is defined by

$$
\Lambda=\frac{h}{\sqrt{2 \pi m k_{B} T}}
$$

where $h$ is Planck's constant and $k_{B}$ is the Boltzmann constant. If $n$ is the spatial density of atoms then the "density in phase space" $\rho$ is the dimensionless quantity

$$
\rho=n \Lambda^{3} .
$$

For spinless particles confined to a box BEC occurs when $\rho=2.612$.

In addition to investigating the fundamental behaviour of the MOT, this thesis was concerned with understanding how to optimize the phase-space density in the MOT and in a variant of the MOT, the "dark" MOT. Both the MOT and dark MOT had important roles in producing the conditions suitable for efficient evaporative cooling in the recent experiments where BEC was observed in rubidium and sodium [1,22]. Much work has already been done to find the limitations on cooling in the MOT. The restrictions on compression have also been studied but much less thoroughly. It was hoped that with careful and systematic measurements of the MOT density it would be possible to identify the limiting mechanism, and hence find a way to increase the compression. In fact it was found that a number of separate processes combine to produce limits on the phase-space density and a model will be presented that generalizes existing treatments of the MOT to describe its behaviour over a wide range of parameters. The understanding gained from this study played an important part in successfully implementing the dark MOT for caesium.

The thesis is organized in the following way. In the remainder of this chapter there is a brief explanation of the mechanism of the MOT and a summary of its historical study which puts in context the experimental work presented here. Descriptions of the basic MOT have appeared many times previously and so that given here is intended only to provide the reader with sufficient background information to appreciate the concepts in later chapters. In chapter 2 the model describing the different regimes of MOT behaviour is presented with particular regard to the phase-space density that one expects. Conditions in the trap depend on the detuning and intensity of the trapping light, the gradient of the magnetic field and the number of trapped atoms, and the aim is to construct an overall picture with semiempirical laws containing proportionality factors that can be found by experiment. Chapter 3 gives a description of the apparatus used in the experiments and the procedures developed for obtaining accurate measurements of the number, density and temperature of the trapped atoms. The experimental measurements themselves are presented in chapters 4-6. In chapter 4 the model described in chapter 2 is tested directly. The general features of the model were confirmed and measurements supplied the required scaling parameters and helped to reduce current uncertainties in various MOT parameters. Accurate measurements were obtained for the first time of clouds extending spatially beyond the capture range of the MOT restoring force. Chapter 5 describes a new technique for measuring intratrap collisional loss rates from a MOT, whose values were important for interpreting the density behaviour of the MOT. Normally one expects to observe the effects of 
such collisions only at very low background vapour pressures; the work presented in this chapter shows that such measurements can be made at much higher ("typical") vapour pressures, and roughly to a comparable degree of accuracy as measurements at low pressures. In chapter 6 the application of the dark MOT to caesium is described. The scheme has been show to work in a straightforward way with sodium, allowing most of the atoms to be confined to a hyperfine level which does not interact with the trapping light and which leads to a significantly higher spatial density than in the ordinary MOT. The extension to the heavier alkalis is more complicated. A model for caesium is developed and tested experimentally to show that similar improvements in density are indeed possible. Finally in chapter 7 some concluding remarks are made.

\subsection{The magneto-optical trap}

The MOT consists of a combination of four or more travelling laser waves intersecting at the centre of a quadrupole magnetic field. The most common geometry is that of three mutually orthogonal pairs of standing waves, comprising counter-propagating beams of opposite circular polarizations. This was the configuration used for all the experiments in this thesis.

The MOT was first demonstrated experimentally by Raab et al. [71] using sodium atoms. The early studies of the MOT were interpreted using a "Doppler" model, in which the force on a trapped atom arises from the imbalance in radiation pressures in each of three pairs of laser beams due to differing Doppler and Zeeman shifts of the beam frequencies in the rest frame of the atom. This description is a simple extension of Doppler cooling theory $[37,91]$. Even at this early stage it was recognized that the optical thickness of a trapped cloud is significant in limiting the attainable density $[86,75]$. In 1989 the observation in sodium of molasses temperatures below the Doppler cooling limit [55] invoked the development of "sub-Doppler" cooling theory based on magnetic sublevels and polarization gradients $[20,84]$. The applicability of this polarization gradient cooling to the MOT was initially not obvious. Steane and Foot [78] were the first to demonstrate that the new cooling mechanisms are not disrupted by the magnetic field of the MOT. Their measurements of the spring constant, friction parameter and cloud temperature were not compatible with the Doppler model. Since then Wallace et al. [88] and Kohns et al. [52] have reported sub-Doppler measurements in rubidium, and Höpe et al. [45] have measured sub-Doppler temperatures in a caesium MOT with high magnetic field gradients. Drewsen et al. [25] have recently made many useful measurements on a caesium MOT. Good introductions to the basic operation of the MOT are to be found in [28] and [77].

\subsubsection{Motion-induced orientation}

When an atom moves at low velocity through a standing wave composed of counterpropagating beams of opposite circular polarization, a population imbalance (orientation) can appear in the magnetic sublevels of the ground state parallel to the atomic motion. This causes the counter-propagating waves to be absorbed by different amounts, 
resulting in unbalanced radiation pressures and a net radiation force.

In a MOT comprising circularly polarized beams it is a highly sensitive motioninduced orientation that is primarily responsible for the confining force at the trap centre, and not the imbalance in scattering rates due to the different Zeeman and Doppler shifts (Doppler forces). The radiation force arising from motion-induced orientation was first explained by Dalibard and Cohen-Tannoudji [20] and Ungar et al. [84]. These original calculations were one-dimensional and semiclassical and gave analytical and numerical treatments for the transition $J=1 \rightarrow J^{\prime}=2$ (Dalibard and CohenTannoudji) and a numerical treatment for $J=2 \rightarrow 3^{\prime}$ (Ungar et al.). There have since appeared a number of other calculations for various transitions and in differing numbers of dimensions, attempting to gain physical understanding of the nature of the force in general. Notably Mølmer [63] has made numerical calculations in one, two and three dimensions for $J=1 \rightarrow 2^{\prime}$, and Steane, Hillenbrand and Foot [79] have presented both analytical and numerical one-dimensional calculations for $J \rightarrow J+\Delta J$, for any $J$ and $\Delta J=0, \pm 1$. Most of the treatments reported have been restricted to low saturation of the atomic transition and to small atomic velocities.

This thesis has been concerned with the properties of the radiation force in the MOT, and it therefore seems appropriate that some explanation of the motion-induced orientation mechanism (if only brief) be given. A thorough explanation is given in the seminal paper by Dalibard and Cohen-Tannoudji [20]. A qualitative understanding of the mechanism is obtained as follows. In a $\sigma^{+}-\sigma^{-}$standing wave the laser polarization is everywhere linear, but its direction rotates in a helix about the propagation axis $z$ with pitch $\lambda$, the laser wavelength. The electric field intensity is independent of position and therefore the energy of the light shifts, in particular those of the ground state sublevels, do not vary with $z$. Thus there are no dipole or gradient forces, or equivalently no stimulated redistribution of photons between the counter-propagating beams. (Actually, some limited redistribution is possible in this type of standing wave, as explained by Dalibard and Cohen-Tannoudji, and this introduces a reactive component to the radiation force. However, Steane, Hillenbrand and Foot [79] show that this is always much smaller than the dissipative contribution, i.e. that part due to the population imbalance). To see how a force arises first consider an atom at rest at a position along the helix at which the linearly polarized light is along the direction $x$. The appropriate eigenbasis for the ground state is the set of eigenstates $\left|M_{x}\right\rangle$ of the angular momentum operator $J_{x}$. The $\pi$-polarized light induces two effects. First, the sublevels have light shifts proportional to the square of the Clebsch-Gordan coefficient for the transition $\Delta M=0$, i.e. $C_{M}^{M}$. Secondly, the absorption and emission of light leads to a steady-state ground state population distributed symmetrically among the sublevels, i.e. there is an atomic alignment in the ground state along $x$. The $\pi$-polarization cannot excite Raman transitions between the ground state sublevels and thus the ground state coherences are all zero. In other words, the density matrix describing the ground state sublevels is diagonal in the $\left|M_{x}\right\rangle$ basis and the sublevel populations can be described by rate equations. The steady-state populations can be found simply in terms of the Clebsch-Gordan coefficients by applying the condition of no net transfer of atoms between sublevels to the rate equations. Now, in this alignment each $\left|M_{x}\right\rangle$ basis state 
is composed of equal proportions of $\left|M_{z}\right\rangle$ and $\left|-M_{z}\right\rangle$ so that there is no population imbalance along $z$. There is thus no net scattering force; this is intuitively obvious from the symmetry in $z$ (linear polarization perpendicular to $z$ ).

Now consider the atom to be at rest at a different value of $z$. The same argument above applies but the alignment is induced along a different direction, $x^{\prime}$. That is, the density matrix is now diagonal in the $\left\langle M_{x^{\prime}}\right\rangle$ basis. Therefore, the effect of moving through the polarization field is described in the original $\left|M_{x}\right\rangle$ basis by a coupling of the sublevel states. The crucial point is that for these mixed states $\left\langle J_{z}\right\rangle$ is no longer zero. In other words, the breaking of the symmetry in $z$ caused by motion invokes a population imbalance along $z$, and a net scattering force results. It should be clear from this description that inducing an atomic orientation initially requires the existence of a atomic alignment. The latter requires at least three sub-levels and hence the simplest transition that can exhibit motion-induced orientation is $J=1 \rightarrow 2^{\prime}$.

In the paper by Dalibard and Cohen-Tannoudji [20] the effect of moving through the helical laser polarization is initially considered as a perturbation on the atomic alignment along $x$. This approach allows much physical insight into the orientation mechanism. An atom with a velocity $v$ sees the direction of the linearly polarized light rotating at an angular frequency $k v$, where $k=2 \pi / \lambda$ is the wavevector of the light. Transforming to a frame that moves with the atom and in which the polarization is stationary, there is a perturbing magnetic field directed along $z$ whose magnitude is such that its Larmor precession frequency is equal to $k v$. In other words, extra to the coupling between the atom and the fixed laser polarization there is a term in the Hamiltonian $H^{\prime}=\hbar k v J_{z}$. To permit a perturbative treatment of this term it is assumed that $k v \ll \Delta$, where $\Delta \equiv \Omega^{2} / 2 \delta$ is typical of the magnitude of the ground state light shifts for a Rabi frequency $\Omega$ and a detuning $\delta$ (strictly this expression is the light shift in the limit of large detuning for a transition with a Clebsch-Gordan coefficient of unity). First-order perturbation theory then gives solutions to the ground state optical Bloch equations to first order in $k v / \Delta$, if terms of order $\Gamma k v / \delta \Delta$ can be neglected. Thus the solution applies to large detunings, $\delta \gg \Gamma$, and the low-velocity dissipative part of the radiation force is given exactly. The force is found to be proportional to the ratio of the saturation parameter per beam $s$ to the light shift $\Delta$ :

$$
F_{i o} \sim \hbar k^{2} s \frac{\Gamma}{\Delta} v \quad\left(J=1 \rightarrow 2^{\prime}\right)
$$

which implies that the force is frictional if the light shift is negative, i.e. if the detuning is negative. The frictional force incurred by Doppler forces is $F_{D o p p} \sim \hbar k^{2} s(\Gamma / \delta) v$ [54] and thus $F_{i o} / F_{\text {Dopp }} \sim 1 / s \gg 1$ at low saturation. The form of Eq. (1.3) is easily understood. The factor $\hbar k s$ accounts for the rate of momentum transfer for a given population imbalance. The factor $\Delta^{-1}$ arises from the first order perturbation theory: the degree of mixing of the $\left|M_{x}\right\rangle$ sublevels (and thus the size of the population imbalance) is inversely proportional to the energy splittings of the light shifted levels.

Steane, Hillenbrand and Foot [79] showed that the form of Eq. (1.3) remains unchanged for ligher $J$ transitions, but significantly that the size of the force increases with $J$. This is because with increasing $J$ the light shifts get closer together and the 
difference in the $\sigma^{+}$and $\sigma^{-}$transition rates from the stretched states (whose population difference dominates the imbalance for $J>1$ ) increases. The cumulative effect of this is a force that increases roughly as $J(2 J+1)$.

Coherence produced by the coupling between ground state sublevels for a moving atom is at the heart of motion-induced orientation, and therefore it should be expected that the friction force will become weak when the precession frequency $k v$ of the perturbation $H^{\prime}$ becomes too large to allow these coherences to build up. Coherences are damped naturally at the optical pumping rate, so we would expect the force to be linear in $v$ only when $k v \ll \Gamma^{\prime}$, where $\Gamma^{\prime}$ is the width of the ground state $\left(\Gamma^{\prime}=s \Gamma\right.$ for $s \ll 1)$. The full velocity dependence of $F_{i o}$ is found by numerically solving the optical Bloch equations. Steane, Hillenbrand and Foot [79] found that the velocity capture range, defined as the velocity at which the force has a local maximum, is given roughly by $s \delta / k$, which is equal to $\Delta / k$ at large detunings. This is much larger than the linear region $\Gamma^{\prime} / k$ for large detunings. The capture range was found to decrease slightly with $J$.

\subsubsection{The Doppler MOT}

Polarization gradient cooling works best at low saturation of the atomic transition. In this regime the Doppler theory of the MOT does not give a good description of the trap behaviour. However, the Doppler model is acceptable under the conditions usually used to load the trap and is relevant to atoms in the process of being captured. It can thus be used to predict the capture and escape velocities.

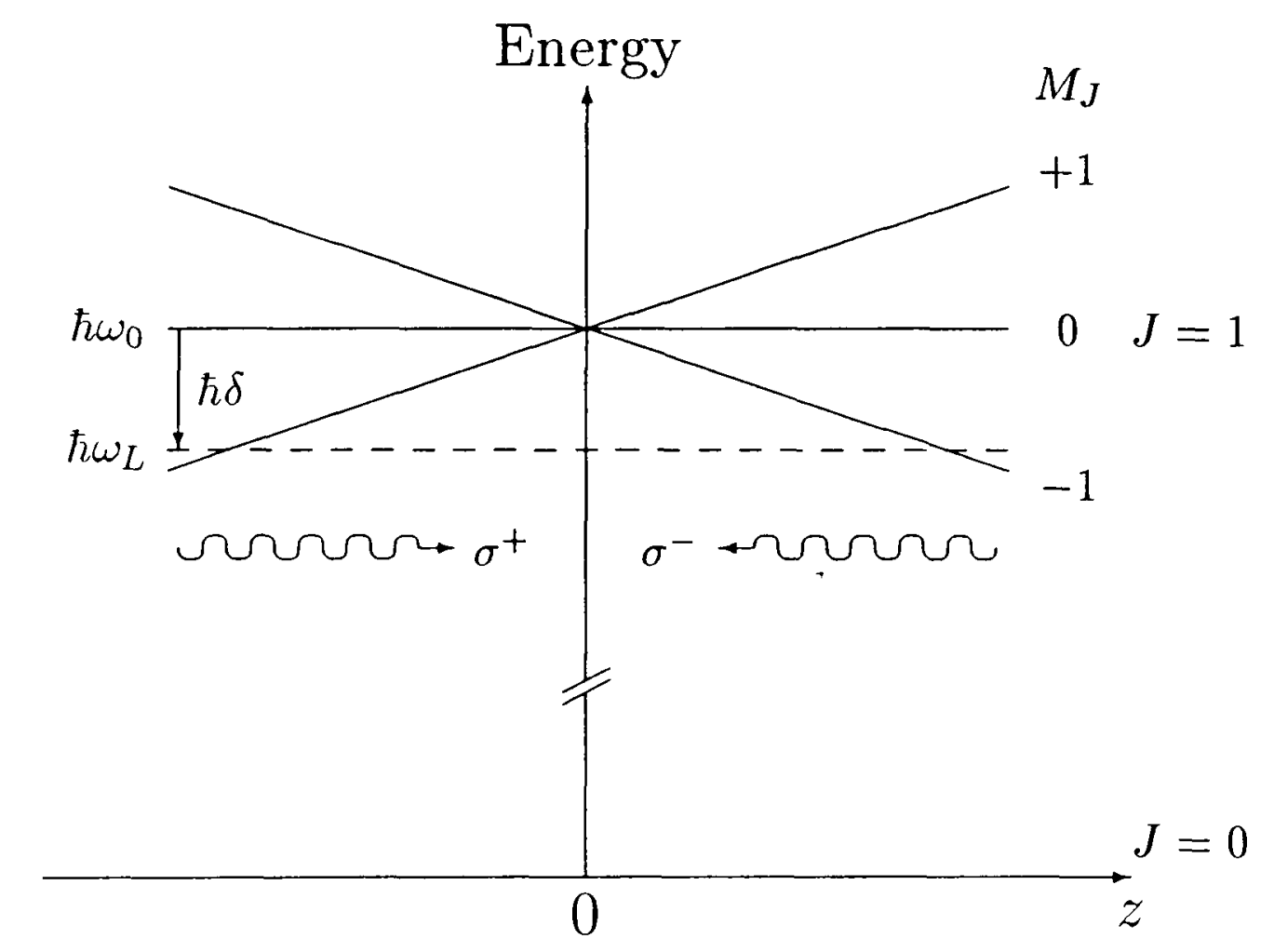

Figure 1.1: Schematic diagram of a one-dimensional MOT for $J=0 \rightarrow 1^{\prime}$.

A simple understanding of the Doppler forces in the MOT is obtained by considering 
the transition $J=0 \rightarrow 1^{\prime}$ in one dimension, shown in Fig. 1.1. The quadrupole magnetic field is assumed to be linear along the $z$ direction over the region concerned, and it removes the three-fold degeneracy of the upper level. For a two-level atom illuminated by a single travelling wave a textbook calculation gives a force due to scattering [57]:

$$
F=\hbar k \frac{\Gamma}{2} \frac{\Omega^{2} / 2}{\Omega^{2} / 2+\Gamma^{2} / 4+\delta^{2}}
$$

where $k$ is the wavevector of the light. For the helicities of the circular polarization shown in Fig. 1.1 the detunings of the $\sigma^{+}$and $\sigma^{-\prime}$ beams in the rest frame of an atom with velocity $v$ and at a position $z$ are respectively

$$
\begin{aligned}
& \delta^{+}=\delta-k v-g \mu_{B} B / \hbar \\
& \delta^{-}=\delta+k v+g \mu_{B} B / \hbar .
\end{aligned}
$$

Thus for $v>0$ and $z>0$ the atom preferentially absorbs the $\sigma^{-}$photons if $\delta<0$ and its motion is thus opposed. The net scattering force produces both velocity and position damping, as one can see by adding the contributions from each beam (valid at low saturation only):

$$
\begin{aligned}
F_{D o p p}=F\left(\delta^{+}\right)-F\left(\delta^{-}\right) & \approx-2\left(k v+g \mu_{B} B / \hbar\right)(\mathrm{d} F / \mathrm{d} \delta)_{\delta=\delta_{0}} \\
& =-\alpha v-\kappa z
\end{aligned}
$$

where $\alpha$ and $\kappa$ are respectively the damping coefficient and spring constant of the trap. The solution to the equation $F_{D o p p}=m \mathrm{~d} v / \mathrm{d} t$ with the boundary condition $v=0$ at $t=0$ and with the assumption of overdamped motion $\left(\alpha^{2} / m \kappa \gg 1\right)$ is

$$
z=A e^{-\kappa t / \alpha}
$$

and thus the position is damped with time $\tau_{\text {pos }}=\alpha / \kappa$.

In three dimensions two coils with currents in opposite directions create a quadrupole field. Since there is cylindrical symmetry, $\nabla \cdot \mathbf{B}=0$ implies $\partial B_{x} / \partial x=\partial B_{y} / \partial y=$ $(1 / 2) \partial B_{z} / \partial z$, where the coil axis lies along $z$. It is then evident from the above discussion that if the laser beams lie along the coordinate axes then there will trapping along these directions. In fact trapping is obtained for all directions in space: a trapping beam will induce $\Delta M=+1,-1$ and 0 transitions for an atom travelling in a general direction, but one can show by resolving the components of the polarization vector that each pair of beams always produces damping, except at right angles to their axis $[77]$.

A MOT is conveniently loaded from a low pressure background vapour [66]. A small fraction of atoms in the tail of the room temperature velocity distribution are slowed sufficiently to be captured by the trapping forces, and they settle at the trap centre. At equilibrium the number of atoms $N$ is determined by a balance between the loading rate $R$ and the total loss rate from the trap, $\Gamma$ :

$$
\begin{aligned}
\frac{\mathrm{d} N}{\mathrm{~d} t} & =R-N \Gamma=0 \\
\Rightarrow N & =R \Gamma^{-1} .
\end{aligned}
$$


When loading from a vapour under typical conditions the dominant loss is by collisions with fast atoms in the vapour itself. Simple kinetic theory gives a collision rate

$$
\frac{1}{\tau}=n_{b} \bar{c} \sigma
$$

where $\bar{c}$ is the mean speed of atoms in the background vapour of number density $n_{b}$ and $\sigma$ is the collision cross-section. This assumes that trapped atom-background atom collisions dominate over collisions with other background species, but that the vapour pressure is not so high that the collision time is shorter than the time for an atom to be captured. This last assumption is true for vapour pressures below about $10^{-7}$ Torr (typically it takes about $50 \mathrm{~ms}$ for a caesium atom to settle to the bottom of the trap). From a simple estimate of the loading rate $R$ [28], Eqs. (1.10) and (1.11) give

$$
\begin{aligned}
N & =R \tau \\
& =C\left(\frac{v_{c}}{\bar{c}}\right)^{4} \frac{d^{2}}{\sigma}
\end{aligned}
$$

where $v_{c}$ is the trap capture velocity, $d$ the dimension of the trapping volume and $C$ a constant roughly equal to $0.2[66,28,77]$. This expression for $N$ is independent of the vapour pressure of the caesium when the assumptions mentioned above are valid, which is generally the case over a pressure range of several orders of magnitude in a high vacuum system.

The velocity capture range for polarization gradient cooling is determined roughly by the linewidth $\Gamma^{\prime}$ of the ground state. However, that for Doppler cooling is determined roughly by the linewidth $\Gamma$ of the excited state. Since usually $\Gamma^{\prime} \ll \Gamma$, Doppler forces generally remain significant at much higher atomic velocities, and for this reason they primarily determine the capture velocity of the MOT. In one dimension the capture velocity is calculated by numerically integrating the equation of motion $F_{D o p p}=m \mathrm{~d} v / \mathrm{d} t$ with suitable boundary conditions. Alternatively one can solve for the motion iteratively by computer [16]. A rough estimate of the capture velocity is found as follows. An atom which surfs into the trap at resonance with the light, i.e. with a velocity such that

$$
k v=g \mu_{B} B / \hbar
$$

experiences the maximum scattering force

$$
F_{\text {Dopp }}^{\max }=\hbar k \frac{\Gamma}{2} \frac{\Omega^{2} / 2}{\Gamma^{2} / 4+\Omega^{2} / 2} .
$$

The capture velocity for a trap radius $r$ is then $v_{\text {cap }}=\left(4 F_{D o p p}^{\max } r / m\right)^{1 / 2}$. For $\delta=-2 \Gamma$, $\Omega=\Gamma, \mathrm{d} B / \mathrm{d} z=15 \mathrm{Gcm}^{-1}$ and $r=5 \mathrm{~mm}$ this gives $v_{\text {cap }} \approx 30 \mathrm{~ms}^{-1}$.

\subsubsection{The sub-Doppler MOT}

If one evaluates $\alpha$ and $\kappa$ for the Doppler MOT one finds that the predicted motion is in fact oscillatory. The overdamped motion that one observes in practice is due to the 
larger friction parameters that result from polarization gradient cooling. In a threedimensional MOT the relative phases of the trapping beams are not controlled and in general there is both Sisyphus cooling [20] and motion-induced orientation. These both contribute to the friction parameter whose detuning dependence is consequently weak [25]. On the other hand, a magnetic field has no first-order effect on Sisyphus cooling and the confining force in the MOT arises from motion-induced orientation only.

The effect of a magnetic field parallel to the counter-propagating $\sigma^{+}-\sigma^{-}$beams in one dimension is readily understood from the Dalibard and Cohen-Tannoudji explanation of motion-induced orientation [20]. In the reference frame rotating with the laser polarization the perturbation is the sum of that due to the fictitious field and the external field:

$$
\begin{aligned}
H^{\prime} & =\hbar k v J_{z}-\mu_{z} B \\
& =\hbar k v J_{z}+g_{J} \mu_{B} B J_{z} \\
& =\hbar k J_{z}\left(v+g_{J} \mu_{B} B / \hbar k\right)
\end{aligned}
$$

In other words, the zero of velocity is shifted and an atom is cooled to the finite velocity

$$
v_{B}=-\frac{g_{J} \mu_{B} B}{\hbar k}
$$

The magnetic field is linear in $z$ and therefore this finite velocity reduces linearly towards the origin, i.e. the effect of the magnetic field is to induce an harmonic force with spring constant

$$
\kappa=\alpha g_{J} \frac{\mu_{B}}{\hbar k} \frac{\mathrm{d} B}{\mathrm{~d} z} .
$$

This equation forms a basis for modelling the spring constant in a real three-dimensional MOT. 


\section{Chapter 2}

\section{Atom-atom interactions in the magneto-optical trap}

\subsection{Introduction}

When the spatial density in the MOT is sufficiently dilute the trapped cloud behaves only as a vapour of neutral atoms. That is, the gas behaviour is ideal except when the atoms undergo short-range collisions. A theoretical understanding of the MOT in this independent-particle regime has been obtained by applying the single-atom theory of laser cooling in one dimension $[14,50,11]$ to the case of a real MOT in three dimensions $[77,25]$. This has given qualitative scaling laws for properties such as the spring constant and the trap radius, and experimental measurements have been used to test the predicted trends and to supply proportionality factors $[77,52,25,88]$. In practice, however, low densities are difficult to obtain in the MOT. Under normal circumstances the optical depth of the trapped cloud is significant and collective effects induced by absorption from and reemission to the light field cause its behaviour to deviate strongly from that of a system of independent atoms. In an early paper by Dalibard [19] it was recognized that attenuation of the incident light by the laser cooled sample itself acts to produce a long-range trapping force. Walker, Sesko and Wieman [86] were the first to realize the significance of long-range repulsive forces arising from photon exchange, so-called "multiple scattering" (or "radiation trapping"). Also of importance are the collisional effects between trapped atoms, which at ultracold temperatures are drastically modified by the presence of a near-resonant laser field ("cold collisions").

The effects of atom-atom interactions in the MOT have generally attracted much interest. Novel spatial distributions of trapped atoms can be induced by the collective effects of multiple scattering, and these have been studied extensively $[86,75,4,36,24]$. Much theoretical and experimental effort has attempted to explain the exotic properties of cold collisions at temperatures below $1 \mathrm{mK}$. There have also been practical incentives for improving understanding of atom-atom interactions. In normal operation both multiple scattering and cold collisions can limit the phase-space density that can be reached in the MOT, and laser cooling methods attempting to attain the conditions necessary for the observation of collective quantum effects have had to address this 
problem. For example, the phase-space density attainable by evaporative cooling from a magnetic trap increases as a function of its value before cooling, and in the recent observation of BEC in rubidium by such a technique [1], optimization of the density in a MOT was a crucial step in the precooling procedure.

It is extremely difficult to combine from first principles the effects of attenuation, multiple scattering and cold collisions, all in the presence of polarization gradients, into a single theoretical modeI [27]. The approach of this experimental thesis has been to combine simple theories with experimental results to produce a set of semiempirical equations describing the MOT. This general technique has been successfully applied to the process of capture in the MOT [56].

This chapter describes the significant atom-atom processes, enabling a simple model of the MOT to be constructed which encompasses the various different regimes of behaviour. Testing of this model was the primary aim of the measurements presented in chapter 4; however, the model is also of direct relevance the cold collision and dark MOT measurements of chapters 5 and 6 respectively. On occasion it was found experimentally that an expected trend of behaviour was not followed; in this instance the experimental observation is briefly mentioned in this chapter and incorporated into the model. The layout of the chapter is as follows. In Sec. 2.2 the model is described and its assumptions explained. The model is applicable to a steady-state operation of the MOT only. Sec. 2.3 considers the effect of suddenly changing the trapping parameters (laser detuning, laser intensity, magnetic field gradient) from one steadystate operation to another; this was the technique used in chapter 4 to attain high phase-space densities. With such a procedure it is possible to obtain useful information about the collisional mechanisms inducing loss in the MOT (chapter 5). Collisional mechanisms are reviewed in Sec. 2.4.

\subsection{The static MOT}

We first consider the MOT to be in a steady-state. That is, the number of atoms trapped remains constant in time, maintained by a dynamic equilibrium between capture and loss processes. Atoms experience a radiation force that can vary significantly with distances comparable to the laser wavelength and on the timescale of the optical pumping time. However, confined atoms typically move over distances on the scale of a millimetre, and the position damping time in the trap is usually much longer than a millisecond. We therefore assume that the atomic motion can be adequately described by a trapping and damping force $f$ that is averaged over several wavelengths and over several cooling transition cycles. It is helpful to consider $\mathbf{f}$ to comprise a single-atom trapping and damping force $\mathrm{f}^{0}$ (i.e. the force in the low density limit), which is then modified by the influence of the other atoms on the local radiation field. Near the trap centre one generally assumes that atoms are slowed sufficiently for $\mathbf{f}^{0}$ to have a linear dependence on the atomic velocity $\mathbf{v}$, so that for small displacements $r$ from the zero of the magnetic field one writes

$$
f_{i}^{0}(\mathrm{r}, \mathrm{v})=-\sum_{j} \kappa_{i j} r_{j}-\sum_{j} \alpha_{i j} v_{j} \quad(\text { small } \mathrm{r}, \mathrm{v})
$$


where $\kappa$ and $\alpha$ are the spring constant and friction tensors of the damped harmonic oscillator. One expects $\kappa_{i i}$ to be proportional to the magnetic field gradient and thus in the magnetic quadrupole field $\kappa_{x x}=\kappa_{y y}=\kappa_{z z} / 2$. At a distance from the trap centre where the Zeeman shift is comparable to the light shift, $\mathrm{f}^{0}$ is not a linear function of $\mathbf{r}$; similarly $\mathbf{f}$ is not a linear function of $\mathbf{v}$ when the Doppler shift $\mathbf{k} \cdot \mathbf{v}$ exceeds the light shift. The non-linearity of the confining force as a function of position is an important feature of the MOT and will be built into the model.

It will not be necessary to make any assumptions about either the friction or the diffusion mechanisms. We merely suppose that their opposing effects balance to yield an equilibrium temperature which, in the absence of atom-atom interactions, links the velocity and position standard deviations $\{\Delta v, \Delta r\}$ via the equipartition theorem:

$$
\frac{1}{2} \kappa_{i i} \Delta r_{i}^{2}=\frac{1}{2} m \Delta v_{i}^{2} .
$$

It is important to appreciate that strictly the equipartition theorem only applies to a system in thermal equilibrium, and that it is not immediately clear that that is the case for a collection of laser cooled atoms. Normally one assigns a temperature to a system when it is in thermal equilibrium with a much larger reservoir; under such circumstances the velocity distribution is always Maxwell-Boltzmann. For a laser cooled vapour there is interaction only with the laser field. Nevertheless, the solution of the Fokker-Planck equation for a friction force proportional to velocity and with small random excursions in momentum space that are independent of velocity (such as those caused by random emission and absorption of photons) is a velocity distribution which is Gaussian [76], and in this respect the assignment of a temperature to optical molasses seems reasonable. The velocity distribution for a damped harmonic oscillator under the same conditions of friction and momentum diffusion is also Gaussian (i.e. the presence of a conservative trapping force does not affect the temperature); further the density distribution is Gaussian, with standard deviation satisfying Eq. (2.2). In practice a measurement of $\kappa$ in the MOT is usually a measurement of the ratio $m \Delta v^{2} / \Delta r^{2}$. In this instance the only assumption made is that the spring constant appearing in Eq. (2.2) is identical to that of Eq. (2.1). That is, it must be ensured experimentally that the restoring force is linear in position over the entire trap volume and that atom-atom interactions are insignificant.

Atom-atom interactions change the local radiation field. In a complete model of such mechanisms it would be necessary to include the effects of cold collision processes, modified by the existence of the trapping light field. At ultracold temperatures the timescale for collisions is comparable to the excited state lifetime, and thus the absorption and emission processes are inextricably linked to the collision dynamics and in a complete description of the collision must be modelled together [30, 48]. Two distinct effects arise from cold collisions in the MOT. A collision may give the participants sufficient kinetic energy for them to overcome the trap depth with the result that they are ejected from the trap. In such an instance the collision contributes to "trap loss". Alternatively, a lower energy collision may not transfer sufficient kinetic energy to cause loss, but will contribute to an overall heating of the trap. In the model of the MOT that is to follow it has been possible to account for trap loss in a straightforward 
manner. However, the heating effect of cold collisions is not included. This is not particularly satisfactory. A recent quantum Monte-Carlo simulation has predicted the existence of significant heating at temperatures below the Doppler limit [44, 43].

With the neglect of collisional heating the main effect of atom-atom interaction is to attenuate the incident field and produce a background field of scattered photons [27, 40, 39]. We will consider the influence this has on the average properties of the trapped cloud, such as its temperature and average density, and neglect any effects on its small scale structure [38]. Walker, Sesko and Wieman [86] found that the effect on the density can be explained qualitatively as a modification to $\kappa$ to account for the repulsive force from photon exchange. We use this idea as the basis of our model. We aim to predict behavioural trends which can be tested experimentally; however the MOT is a complicated system of many multi-level atoms interacting with themselves and with a complex light field, and there are many unavoidable inherent uncertainties, in addition to practical difficulties, in measuring the large scale properties of the cloud for a given set of trapping parameters (these problems will be discussed in the experimental chapters). In keeping with the accuracy of measurement that is possible it is intended that the model should enable the trap properties to be derived with an accuracy at roughly the $50 \%$ level.

It will become evident that the behaviour of the MOT can be described in terms of four different regimes. At small numbers of trapped atoms (typically less than $10^{4}$ ) the optical depth of the cloud is very small and multiple scattering effects can be ignored. The atoms act independently. This regime will be termed "temperaturelimited" because the cloud size in the harmonic trapping potential is determined solely by the trap temperature. As more atoms are loaded into the trap scattering effects become significant. In the multiple scattering regime the trap size is determined by long-range repulsive forces. At still higher numbers of atoms, or at high field gradients or low light shifts, the non-linearity of the spatial variation of the confining force is important. A weakening of the spring constant is observed to occur within the spatial distribution of atoms and the trap enters a "two-component" regime. Finally, if the optical depth of the cloud is sufficiently large there is a significant probability that a photon is scattered more than twice before it escapes the cloud. This fourth regime occurs for high numbers of atoms (typically for greater than $10^{8}$ atoms at a high scattering rate), and is discussed in [56] and [51]. The regime was just accessible in our experimental measurements, but was not of majör concern and is only discussed briefly.

The following definitions will be used. The absolute value of the detuning $\left|\omega_{L}-\omega_{0}\right|$ of the laser frequency with respect to the atomic resonance frequency is denoted by $\delta$. In all the experiments a "red" detuning, $\omega_{L}<\omega_{0}$, was used. The natural linewidth (FWHM) of the atomic excited state $6 \mathrm{P}_{3 / 2}$ in caesium is $\Gamma=2 \pi \times(5.22 \pm 0.01) \mathrm{MHz}$ [72]. The definition of the Rabi frequency $\Omega$ in a light field of intensity $I$ is taken to be

$$
\frac{\Omega^{2}}{\Gamma^{2}}=\frac{I}{I_{S}}
$$


where for an electric dipole transition in a two-level atom the saturation intensity $I_{S}$ is

$$
I_{S}=\frac{1}{3} \hbar c k^{3} \frac{\Gamma}{2 \pi}
$$

(where $\hbar$ is Planck's constant divided by $2 \pi, c$ is the speed of light and $k=2 \pi / \lambda$ is the wavevector of the light). Eq. (2.4) is directly applicable to the $6 \mathrm{~S}_{1 / 2} F=4, M_{F}=4 \rightarrow$ $6 \mathrm{P}_{3 / 2} F^{\prime}=5, M_{F^{\prime}}=5$ transition in caesium because decay via the electric dipole from $F^{\prime}=5$ can only be to $F=4$ (i.e. the $F=4 \rightarrow 5^{\prime}$ transition is a good approximation to a two-level system) and because the Clebsch-Gordan coefficient between stretched magnetic sublevels is unity. The formula gives $I_{S}=2.24 \mathrm{~mW} / \mathrm{cm}^{2}$. Throughout $\Omega$ will be used to refer to the Rabi frequency of a single laser beam; $\Omega_{t o t}$ denotes that from all six trapping beams.

\subsubsection{Temperature-limited regime}

If there is only a small probability that a photon scattered from a trapping beam will interact with a second atom before leaving the trap, then multiple scattering effects can be neglected. This is the case for small numbers of trapped atoms or low saturation of the atomic transition. In this regime the gas behaves as a collection of $N$ independent atoms in a harmonic potential. The validity of assigning a temperature to this system has already been discussed; the spatial distribution is Gaussian with r.m.s. radius along each axis given by the equipartition theorem:

$$
\frac{1}{2} \kappa_{i i} r_{i, T}^{2}=\frac{1}{2} k_{B} T
$$

where the subscript " $T$ " is to emphasize the role of the trap temperature in fixing the trap dimensions. In a magnetic quadrupole field $\kappa_{z}=2 \kappa_{x}=2 \kappa_{y}$, and thus $r_{z}=$ $r_{x} / \sqrt{2}=r_{y} / \sqrt{2}$ since the temperature is assumed to be isotropic (see Salomon et al. [73] for a brief experimental discussion of temperature isotropies in optical molasses). Defining $r_{z} \equiv r_{T}$ and $\kappa \equiv \kappa_{z}$ the peak spatial atomic density for $N$ atoms (at the centre of the ellipsoidal Gaussian) is

$$
n_{T}=\frac{N}{2\left(\sqrt{2 \pi} r_{T}\right)^{3}} .
$$

Strictly one should not expect the distribution to be exactly ellipsoidal because in three dimensions $\pi$-transitions reduce the confinement along off-axis directions [71, 77]. An important feature of Eq. (2.6) is that since the temperature is independent of the number of atoms, the cloud density is proportional to number. This is not the case in the other regimes to be discussed. From Eqs. (1.2), (2.5) and (2.6) the density is phase space is

$$
\rho=\frac{N \hbar^{3}}{2 k_{B}^{3} T^{3}}\left(\frac{\kappa}{M}\right)^{3 / 2}
$$

which thus increases rapidly with decreasing temperature. 
The origin of the spring constant $\kappa$ in the limit of low spatial density was described in the Introduction. A magnetic field has a first-order effect only on motion-induced orientation. Optical pumping among the Zeeman sublevels of the ground state induces a population imbalance along the direction of motion which cools to a finite, rather than zero, velocity. This finite velocity is proportional to the magnetic field and thus distance from the origin in a quadrupole field. The result is a harmonic confining force characterized by a spring constant $\kappa$ proportional to the friction parameter $\alpha$. A semiclassical expression for $\kappa$ was first derived in one dimension by Steane and Foot [78] (see also Steane, Chowdhury and Foot [77] and more recently Drewsen et al. [25]). They found a dependence independent of laser intensity and inversely proportional to detuning for detunings larger than a couple of linewidths:

$$
\begin{aligned}
\kappa & =\alpha g_{F} \frac{\mu_{B}}{\hbar k} \frac{\mathrm{d} B}{\mathrm{~d} z} \\
& =\kappa_{0} \frac{\Gamma}{\delta} \frac{b}{b_{0}} \quad(\text { for } \delta \gtrsim 2 \Gamma)
\end{aligned}
$$

where $g_{F}$ is the Lande $\mathrm{g}$-factor of the ground state, $b \equiv d B / d z$ is the gradient of the magnetic field, $\kappa_{0}$ is a constant of proportionality, and we will define $b_{0} \equiv 1 \mathrm{Gcm}^{-1}$. The form of Eq. (2.8) applies to any $J \rightarrow J^{\prime}=J+1$ transition [79] and has been validated by a $J=4 \rightarrow 5^{\prime}$ quantum analysis in one dimension by Drewsen et al. [25].

The extension of Eq. (2.8) to a real three-dimensional trap is difficult because of the inevitable existence of spatial variations in the light shifts and optical pumping rates [77]. The pattern of polarization gradient is in general not optimal for motion-induced orientation (i.e. some degree of Sisyphus cooling is always present) but crucially the pattern is also alignment-sensitive. Despite this, measurements on three-dimensional traps have been in general agreement with the dependence of Eq. (2.8). In Table 2.1 the reported measurements of $\kappa$ have been converted into measurements of $\kappa_{0}$. There is a wide variation in these values. This is at least partly symptomatic of the large systematic errors associated with each measuring technique, which are now briefly discussed.

The scattering force from an additional "pushing" laser will displace the trap to a position where its effect is balanced by the harmonic restoring force. A measurement of the displacement and a knowledge of the pushing force thus allows $\kappa$ to be deduced. However, the force is difficult to calculate accurately because of the uncertainty in the orientation and optical pumping effects in three dimensions [78]. As shown by Steane, Chowdhury and Foot [77] a more calculable effect is the pushing obtained by introducing a small intensity imbalance into one of the trapping directions. In the limit of low saturation the force is straightforwardly calculated in one dimension from the motion-induced orientation equations of Dalibard and Cohen-Tannoudji [20]. However, there still remain systematic uncertainties in the knowledge of parameters such as the Rabi frequency; furthermore, the effect of mixed polarization in three dimensions still introduces some uncertainty.

A measurement of the trap radius at given temperature allows $\kappa$ to be deduced directly from Eq. (2.5). However, to be sure that the trap is indeed temperature- 
Table 2.1: Reported measurements of $\kappa$, converted into values of $\kappa_{0}$ using Eq. (2.8) to allow direct comparison. In addition to the experimental results two theoretical values are given for "Cs" - these are the results of a semi-classical, three-dimensional numerical calculation for an idealised atom with parameters appropriate for caesium.

\begin{tabular}{lrlll}
\hline \hline $\begin{array}{l}\text { value of } \kappa_{0} \\
\left(10^{-19} \mathrm{~N} / \mathrm{m}\right)\end{array}$ & atom & transition & method & reference \\
\hline 0.4 & $\mathrm{Na}$ & $2 \rightarrow 3$ & "pushing" laser & Raab et al. [71] \\
0.8 & $\mathrm{Cs}$ & $4 \rightarrow 5$ & "pushing" laser & Sesko, Walker, Wieman [75] \\
0.4 & $\mathrm{Cs}$ & $4 \rightarrow 5$ & measured $r \& T$ & Drewsen et al. [25], fig 14 \\
1 & $\mathrm{Cs}$ & $4 \rightarrow 5$ & measured $r \& T$ with few atoms & Drewsen et al. [25], table 1 \\
0.5 & $\mathrm{Cs}$ & $4 \rightarrow 5$ & imposed oscillating $B$ field & Höpe et al. [45] \\
2.7 & $\mathrm{Cs}$ & $4 \rightarrow 5$ & beam imbalance & Steane, Chowdhury, Foot [77] \\
3.0 & "Cs" & $1 \rightarrow 2$ & numerical calculation & Mølmer [62] \\
3.3 & ${ }^{8 \mathrm{Cs} \text { " }}$ & $4 \rightarrow 5$ & numerical calculation & Mølmer [62] \\
0.2 & ${ }^{85} \mathrm{Rb}$ & $3 \rightarrow 4$ & imposed oscillating $B$ field & Kohns et al. [52] \\
2.5 & ${ }^{85} \mathrm{Rb}$ & $3 \rightarrow 4$ & measured $r \& T ;$ beam imbalance & Wallace et al. [88] \\
2.0 & ${ }^{87} \mathrm{Rb}$ & $2 \rightarrow 3$ & measured $r \& T$; beam imbalance & Wallace et al. [88] \\
\hline \hline
\end{tabular}

limited it is necessary to retain only a very small number of atoms. This reduces the fluorescence signal and can make measurement of the trap dimensions difficult.

Finally, $\kappa$ has been cleverly measured by monitoring the phase response of trap oscillations to an external oscillating force and comparing it to that expected for an overdamped harmonic oscillator $[52,45]$. In this method care must be taken to confine the displacement of the trap to the region of space where $\kappa$ varies linearly with distance.

In summary, therefore, there are difficulties with all these methods, and experimental determination of $\kappa$ still remains somewhat problematic. In addition it is not known how sensitive $\kappa_{0}$ is to the exact pattern of polarization gradient in three dimensions. A reasonable knowledge of the absolute value of the confining force is an important requirement for a model of the density, and for this reason it was felt necessary to make our own measurement of $\kappa$.

The non-linearity of the spring constant was found to be an important issue in our experimental work. The spatial breakdown in $\kappa$ is the origin of the two-component regime and will be discussed in detail in Sec. 2.2.3. Also of importance is a breakdown in $\kappa$ at low light shifts. One might expect a reduction in $\kappa$ below that predicted by Eq. (2.8) at a similar light shift to that at which the friction force begins to decrease (Sec. 1.1.1). However, a recent semiclassical calculation in one dimension [25] suggests that the breakdown in confinement follows more closely that of the temperature, i.e. the breakdown of the ratio $D / \alpha$. This is at odds with the measurements of Wallace et al. [88]. They observed a minimum of temperature at a much lower light shift than that at which the spring constant started to decrease and attributed this to the breakdown in diffusion allowing cooling to a much lower temperature, i.e. $D$ and $\alpha$ begin to fall off (at least initially) at roughly the same rate, and thus their ratio remains small.

An early experimental study [78] showed that the temperature in a caesium MOT at low atomic density is similar to that in the corresponding molasses, i.e. to that obtained 
in the same physical system but with zero magnetic field gradient. More recently two experimental studies $[25,88]$ have confirmed that the temperature in the low density MOT is indeed identical to that in a molasses of the same laser field configuration (at least for field gradients of order $10 \mathrm{Gcm}^{-1}$ ). For detunings larger than a few linewidths and light shifts in the range indicated, the temperature in three-dimensional optical molasses varies for circularly polarized beams in the same way as for linearly polarized beams:

$$
k_{B} T=C_{0}+C_{\sigma} \hbar \frac{\Omega^{2}}{\delta} \quad\left(\delta \gtrsim 4 \Gamma, 0.05 \Gamma \lesssim \Omega^{2} / \delta \lesssim 0.4 \Gamma\right) .
$$

The expectation of this variation stems from the original semiclassical prediction in one dimension by Dalibard and Cohen-Tannoudji [20]; subsequently Mølmer showed (also semiclassically) that the scaling remains unchanged in three dimensions [63]. Wallace et al. [88] have verified the form experimentally for both ${ }^{85} \mathrm{Rb}$ and ${ }^{87} \mathrm{Rb}$ and obtained quantitative agreement with the latter with a semiclassical calculation that averages over the relative phases of the molasses beams. Drewsen et al. [25] have provided experimental verification for caesium.

It was felt necessary to make our own measurements of the temperature in a caesium $\sigma^{+}-\sigma^{-}$molasses for two reasons. First, agreement with the variation expected is a good test of correct fundamental trap behaviour, and allows the boundary between the temperature-limited and multiple scattering regimes to be established. Secondly, quantum Monte-Carlo wavefunction analysis of three-dimensional optical molasses [12, $62]$ has recently given excellent quantitative agreement with the lin $\perp$ lin and $\sigma^{+}{ }_{-}^{-}$ measurements on rubidium [31, 88], and the lin $\perp$ lin measurements on caesium [73], but not the $\sigma^{+}-\sigma^{-}$data of Drewsen et al. [25]. The results of the measurements are described in chapter 4 but are quoted here in order to use them to build our semiempirical model. The results of Drewsen et al. [25] were verified. A slight departure from a linear dependence on the light shift was observed for values of $\Omega^{2} / \Gamma \delta$ between 0.05 and 0.4. Nevertheless a straight line could be fitted through all the data for $0.05<$ $\Omega^{2} / \Gamma \delta<0.4$ (i.e. temperatures up to about $30 \mu \mathrm{K}$ ), to obtain $C_{0} / k_{B}=1 \pm 0.5 \mu \mathrm{K}$, $C_{\sigma}=0.28 \pm 0.05$. In the following this pair of values for $C_{0}$ and $C_{\sigma}$ is used.

When the constant term in Eq. (2.9) can be neglected, $T \propto \Omega^{2} / \delta$, which from Eqs. (2.9), (2.8) and (2.5) implies a cloud radius independent of detuning and number, and proportional to the Rabi frequency $[77,25]$ :

$$
r_{T}=\sqrt{\frac{\hbar C_{\sigma} \Gamma}{\kappa_{0}}} \frac{\Omega}{\Gamma} \sqrt{\frac{b_{0}}{b}} .
$$

\subsubsection{Multiple scattering regime}

As atoms are loaded into a temperature-limited MOT the density increases according to Eq. (2.6). Eventually when the number becomes sufficiently high (typically at about $10^{4}$ atoms, but strongly dependent upon the scattering rate) the reabsorption of scattered photons within the cloud limits the density. Walker, Sesko and Wieman [86] were the first to recognize the significance of photon exchange in the MOT. They observed that as atoms were loaded at high density the cloud size grew rapidly to reach 
sizes that did not remotely correspond with the behaviour of an ideal gas for the trap temperatures that were measured. Moreover, the density remained almost independent of number and the spatial distribution was uniform rather than Gaussian. They interpreted this behaviour as being due to long-range repulsive forces, and proposed a simple model which led to a quantitative expression for a multiple scattering-limited density, independent of number. Their general arguments, which are incorporated into our model, were vindicated by the observation of unusual spatial distributions when the trapping beams were slightly misaligned, in particular the formation of a ring of atoms, or part of a ring of atoms, orbiting about a small central cloud at well-defined radii. The existence of stable orbits at discrete radii was predicted by their model only when the torque resulting from the beam misalignment was combined with the effect of long-range repulsion.

In the multiple scattering regime the restoring and friction forces are still produced by sub-Doppler mechanisms as in the low density limit, but the mechanisms are themselves modified. In the Walker, Sesko, Wieman model, the restoring force for an atom remains simple harmonic but is opposed by a force arising from the cumulative effect of inverse-square repulsions between pairs of atoms. In this picture the mechanism by which the repulsive force arises relies entirely upon the modification of absorption and emission profiles by the laser field. This can be understood intuitively with the following description. Of interest is how the process of scattering at one point in the cloud can contribute to the generation of a net repulsive force $\mathbf{F}_{\text {rep }}$ at a different point in the cloud. To prevent the cloud collapsing to the temperature-limited density given by Eq. (2.6) we stipulate that $\mathbf{F}_{r e p}(\mathbf{r})$ balances the harmonic restoring force $-\kappa \mathbf{r}$. There are two related mechanisms by which long-range forces are exerted. The scattering of a photon out of a trapping beam (attenuation) causes a beam imbalance which on average tends to compress the cloud [19]. On the other hand if the scattered photon is subsequently reabsorbed then there is a momentum recoil which on average tends to expand the cloud. There is a net repulsion force only if the latter exceeds the former; it is now argued that this only happens because some of the light is scattered inelastically. According to Mollow [61] scattered light in a laser field has both elastic (Rayleigh) and inelastic (fluorescence) contributions, with an intensity ratio that is the saturation parameter $s$ :

$$
\frac{I_{\text {inel }}}{I_{e l}}=s .
$$

Consider first the elastic component. The average scattering force on an atom is the net absorption rate times the photon momentum, and thus because elastic scattering only redistributes photon momentum flux spatially and does not change its total value, and because the cross-section for absorption of an elastic photon is identical to that for absorption of a laser photon, one must conclude that the divergence of any force that elastic scattering produces is zero. A repulsive force with zero divergence is not consistent with the symmetry of a force that balances $-\kappa \mathbf{r}$ in all directions, and therefore one must conclude that $\mathbf{F}_{\text {rep }}$ must derive from the inelastic component of the scattered light. For moderate light shifts $\left(\Omega^{2} / \delta \Gamma \lesssim 1\right)$ the fluorescence spectrum of spontaneously emitted (inelastic) light takes the form of three Lorentzians symmetrically positioned 
about the laser frequency, the Mollow triplet [61]. The most important consequence of this spectral redistribution is a change in the absorption cross-section. The highest frequency component of the Mollow triplet lies close to the transition frequency, and its absorption thus dominates the cross-section. For this reason the cross-section for absorption of a reradiated photon $\sigma_{R}$ is larger than that for absorption of a laser photon $\sigma_{L}$. The compression force from trapping beam attenuation is proportional to the former; the repulsive force from reabsorption is proportional to the latter and consequently there is net repulsion.

The argument above is similar to that invoked by Walker, Sesko and Wieman [86]. In a few simple steps they derived the following expression:

$$
\nabla \cdot \mathbf{F}_{r e p}(\mathbf{r})=\frac{I_{t o t}}{c} n_{\mathrm{MS}}(\mathbf{r}) \sigma_{L}^{2}\left(\frac{\sigma_{R}}{\sigma_{L}}-1\right)
$$

where $n_{\mathrm{MS}}(\mathbf{r})$ is the spatial density in the multiple scattering regime, $I_{\text {tot }}$ is the total light intensity, and where, as from the description above, the magnitude of $\mathbf{F}_{\text {rep }}$ does indeed depend on the difference $\sigma_{R}-\sigma_{L}$. By applying the divergence theorem to this expression it is clear that $\mathbf{F}_{\text {rep }}(\mathbf{r})$ only depends on the total number of atoms inside the sphere of radius $r$. Thus a particular solution for $n(\mathbf{r})$ is the uniform density distribution $n$ that was observed experimentally. In this instance equating $\nabla \cdot \mathbf{F}_{r e p}(\mathbf{r})$ to $\nabla \cdot(\kappa r)$ for a balance of forces predicts finally

$$
n=\frac{3 \kappa c}{I_{t o t} \sigma_{L}^{2}\left(\sigma_{R} / \sigma_{L}-1\right)} .
$$

The behaviour of the density is thus predicted from a knowledge of $\kappa$ and the ratio $\sigma_{R} / \sigma_{L}$

It is important to remark upon the assumptions of the Walker, Sesko, Wieman model. It is assumed that the probability that a photon is scattered more than twice inside the cloud is insignificant. If this is not the case then there is a different density regime (to be described briefly later). The splitting of the atom-atom interaction into repulsive and attractive parts has been formally justified by Ellinger, Cooper and Zoller [27]. An important limitation is the inherent assumption that the forces between two atoms fall off as the inverse square of their separation (this $1 / r^{2}$ dependence of course leads to the divergence of the force being proportional to $n(\mathbf{r})$, the force source density). This only holds in the far-field limit, that is, when the mean separation of the atoms is much greater than the laser wavelength $[27,40]$; at smaller separations $1 / r$ terms become significant. Thus the model is of questionable value in caesium for densities of the order of $10^{12} \mathrm{~cm}^{-3}$ and larger.

One would proceed to evaluate Eq. (2.13) in the following way. In the first instance one assumes $\kappa$ is given by Eq. (2.8). One then needs to find $\sigma_{R} / \sigma_{L}$. This ratio was calculated numerically in [86] by convolving the emission and absorption spectra of a two-level atom in a one-dimensional standing laser wave. Steane, Foot and Chowdhury [77] produced an analytical approximation for the same situation. In [83] we reported an improvement upon the estimate in [77]. Consider a two-level atom illuminated by a single travelling wave. The fluorescence spectrum in the limit $\Omega_{t o t}, \delta \gg \Gamma$ can be 
deduced using the dressed atom approach [15]. This leads to a complicated expression for $\sigma_{R}$ which can be fitted to better than $30 \%$ accuracy for $\Omega_{t o t}, \delta \gg \Gamma$ by the formula

$$
\frac{\sigma_{R}}{\sigma_{L}}-1=\frac{\Omega_{t o t}^{2}}{2 \Gamma^{2}} \frac{\delta^{2}-2 \Gamma^{2}}{\delta^{2}+\Omega_{t o t}^{2}} .
$$

Substituting this into Eq. (2.13) and making use of Eq. (2.8) yields the following expression for the MOT density in the presence of multiple scattering of photons:

$$
\begin{aligned}
n_{\mathrm{MS}} & =\kappa \frac{\left(4 \delta^{2}+\Gamma^{2}+12 \Omega_{t o t}^{2}\right)^{2}}{18 \lambda \hbar \Gamma \Omega_{t o t}^{4}}\left(\frac{\delta^{2}+6 \Omega_{t o t}^{2}}{\delta^{2}-2 \Gamma^{2}}\right) \\
& \approx \frac{8}{9} \frac{\kappa_{0}}{\lambda \hbar \Gamma} \frac{b}{b_{0}} \frac{\Gamma \delta^{3}}{\Omega_{t o t}^{4}} \quad \text { if } \quad \frac{\Omega_{t o t}^{2}}{\delta^{2}} \ll 1 .
\end{aligned}
$$

The important prediction is that the density should increase rapidly with decreasing saturation of the atomic transition. This result is largely independent of the details of calculating $\sigma_{R} / \sigma_{L}$. For example consider the case of large detuning. The cross-sections $\sigma_{R}$ and $\sigma_{L}$ differ because the scattered light has an inelastic component. According to Eq. (2.11) the fraction of the scattered intensity which is inelastic falls of as $\Omega_{\text {tot }}^{2} / \delta^{2}$ at large detuning. However, the cross-section for the inelastic light is roughly a factor $\delta^{2} / \Gamma^{2}$ larger than that for the elastic light because the former lies approximately at the transition frequency. Thus one would expect $\left(\sigma_{R} / \sigma_{L}-1\right)$ to approach a constant $\sim \Omega_{\text {tot }}^{2} / \Gamma^{2}$ at large detunings, and this is indeed the prediction of Eq. (2.14). It is then clear from Eq. (2.13) that the detuning dependence of $n_{\mathrm{MS}}$ is that of $\kappa / \sigma_{L}^{2}$, i.e. $\delta^{3}$. In other words, the density increases dramatically at low saturation because the repulsive force decreases rapidly due to its reliance on the probability of two scattering events. As a further point, it is interesting to note that the denominator in Eq. (2.15) becomes zero when $\delta=\sqrt{2} \Gamma$, i.e. the existence of multiple scattering is tending to compress the cloud. This is again understood from the Mollow triplet. At small detunings the highest frequency component of the triplet is actually further from resonance than the near resonant light field.

One would perhaps not expect the form of Eq. (2.15) to reproduce accurately the density behaviour for the real case of caesium (with multiple magnetic sublevels) in a three-dimensional laser field. However, the properties described in the preceding paragraph are argued in a fairly general way and one might hope for their observation. In fact it was found that Eq. (2.15) does not predict at all well the variation in density under different scattering conditions. For possible reasons that will be discussed later the dependence of $n_{\mathrm{MS}}$ on $\delta$ and $\Omega$ was found to be much weaker than the prediction; for detunings above a linewidth or so the variation was roughly $n_{\mathrm{MS}} \propto b \delta^{0.5} \Omega^{-1}$ :

$$
n_{\mathrm{MS}}=C_{\mathrm{MS}} \frac{\kappa_{0}}{\lambda \hbar \Gamma} \frac{b}{b_{0}}\left(\frac{\delta \Gamma}{\Omega^{2}}\right)^{1 / 2}(\delta \gtrsim \Gamma)
$$

where $C_{\mathrm{MS}}$ is a dimensionless parameter whose value is given by experiment, and where the other constants have been chosen to allow easy comparison with Eq. (2.16). The parameter $\kappa_{0}$ has been included because it is expected that if the MOT has a large 
spring constant in the temperature-limited regime, then it will produce proportionately large densities in the multiple scattering regime. However, no other assumptions concerning the scaling of the spring constant in the multiple scattering regime are made, other than that it is proportional to the magnetic field gradient.

The prediction that the density in the multiple scattering regime is independent of number was found to be fairly good. However, uniform densities were only observed when the scattering rate was high and the magnetic field gradient low; for the most part the spatial distribution was well approximated by a Gaussian. We therefore choose that $n_{\mathrm{MS}}$ represent the peak density of a Gaussian distribution. Further, since the density is now proportional to $b$, one might expect a radius scaling as the cube root of $b$, which would imply that the ellipticity of the trapped cloud is reduced compared with the temperature-limited regime [c.f. Eq. (2.10)]. We assume therefore that we can approximate the cloud as a spherical distribution. The cloud volume is now dictated by the density, and for $N$ atoms has an r.m.s. radius given by

$$
r_{\mathrm{MS}}=\frac{1}{\sqrt{2 \pi}}\left(\frac{N}{n_{\mathrm{MS}}}\right)^{1 / 3}
$$

This is to be compared with the radius $r_{T}$ given by equation (2.5). These two radii define the boundary between the temperature-limited and multiple scattering regimes. When $r_{\mathrm{MS}}>r_{T}$, the cloud is not compressed to the temperature-limited radius, but remains fatter due to the multiple photon scattering.

In the Sesko, Walker, Wieman study [75] the MOT temperature was observed to rise as the number of trapped atoms was increased. It is now well established that multiple scattering also causes the temperature of atoms to be different from that at low densities. Two experimental studies have shown MOT temperatures increasing above the molasses temperature by an amount proportional to the cube root of the total number of trapped atoms [25, 17], although the exact dependence on the intensity and detuning is still somewhat uncertain. Multiple scattering induces excess heating by both enhancing the momentum diffusion and reducing the efficiency of polarization gradient cooling (thereby lowering the friction force). The $N^{1 / 3}$ dependence has been verified by one-dimensional calculations $[27,40,39]$ but a model for the variation with $\Omega$ and $\delta$ has yet to be fully worked out. These additional effects of multiple scattering are of some relevance to densities. First, it would be of interest to know whether the degradation of the spring constant by multiple scattering is in part responsible for the failure of Eq. (2.15). It was possible to address this issue by the temporal method of measuring densities (Sec. 2.3 and chapter 4). Secondly, it will be shown later that multiple scattering limits the density before it significantly raises the temperature, so it will not be necessary to take the temperature rise into account when establishing the boundary between the temperature-limited and multiple scattering regimes.

\subsubsection{Two-component regime}

The restoring force in the MOT is not a linear function of position over the whole trapping region. Near the trap centre the effect of the linearly varying magnetic field 
is to modify the motion-induced orientation in such a way as to produce harmonic confinement. However, outside this region the magnetic field is a large perturbation on the process of optical pumping among the ground state magnetic sublevels and the efficiency of the polarization gradient cooling is reduced, resulting in a weakening of the restoring force. At large displacements from the trap centre only the Zeeman shifts of various transitions to the excited state can provide confinement.

The non-linear variation with position of the MOT restoring force was observed by Steane, Chowdhury and Foot [77] during intensity imbalance measurements. They noticed that when the trap was displaced far from the trap centre (i.e. at large imbalances) the brightest region of the cloud remained fixed in position while a fan of atoms extended out into the region of higher magnetic field. This dispersive behaviour in the higher field was cited as evidence for the breakdown of motion-induced orientation. One would expect the non-linearity of the restoring force to have the following effect on the spatial distribution in the MOT. When the trap is loaded with a small number of atoms just the central, strongly confined region is filled. As more atoms are loaded the trap passes into the multiple scattering regime and starts to expand at (roughly) constant density. Eventually the central region is filled and further atoms then spill over into the weakly confining surrounding volume, spreading out to a much larger radius. With a sufficiently large number of atoms, almost all the atoms occupy this surrounding volume and the trap behaviour is predominantly determined by Doppler forces, i.e. the presence of polarization gradients in the laser field no longer determines the average temperature and density. The signature of the two-component regime is thus a density distribution consisting of two components: a diffuse Gaussian ball extending out to a large radius, with a small, much more dense collection of atoms in the centre. From the observations of Steane, Chowdhury and Foot [77] it is expected that the diffuse ball should be sensitive to alignment and intensities of the trapping beams, while the central region should not.

The boundary between the central and outer regions occurs at a radius such that the Zeeman and light shifts in the ground state are roughly equal. This condition is easily understood in one dimension. In the Dalibard and Cohen-Tannoudji picture of motion-induced orientation [20], the effect of the rotating linear laser polarization is replaced by a fictitious magnetic field in the reference frame in which the polarization is stationary, and its magnitude is such that the corresponding Larmor precession frequency is equal to the Doppler shift of the atom $k \dot{v}$. Steane, Hillenbrand and Foot [79] showed that the capture velocity $v_{c}$ of motion-induced orientation (defined as the velocity at which the force has a local maximum) is given roughly by $k v_{c}=\Delta$, i.e. the velocity at which the Doppler shift equals the light shift of the ground state. One may qualitatively think of this as the velocity above which the Larmor precession frequency of the magnetic field perturbation is too large to allow the ground state coherences to build up. Thus in the presence of a real magnetic field one should expect the motioninduced orientation to break down when its Larmor frequency equals the ground state light shift, or in other words, when the Zeeman shift it induces equals the light shift. A fully quantum mechanical calculation in one dimension has verified this semiclassical argument [90]. Results of preliminary semiclassical calculations have predicted the 


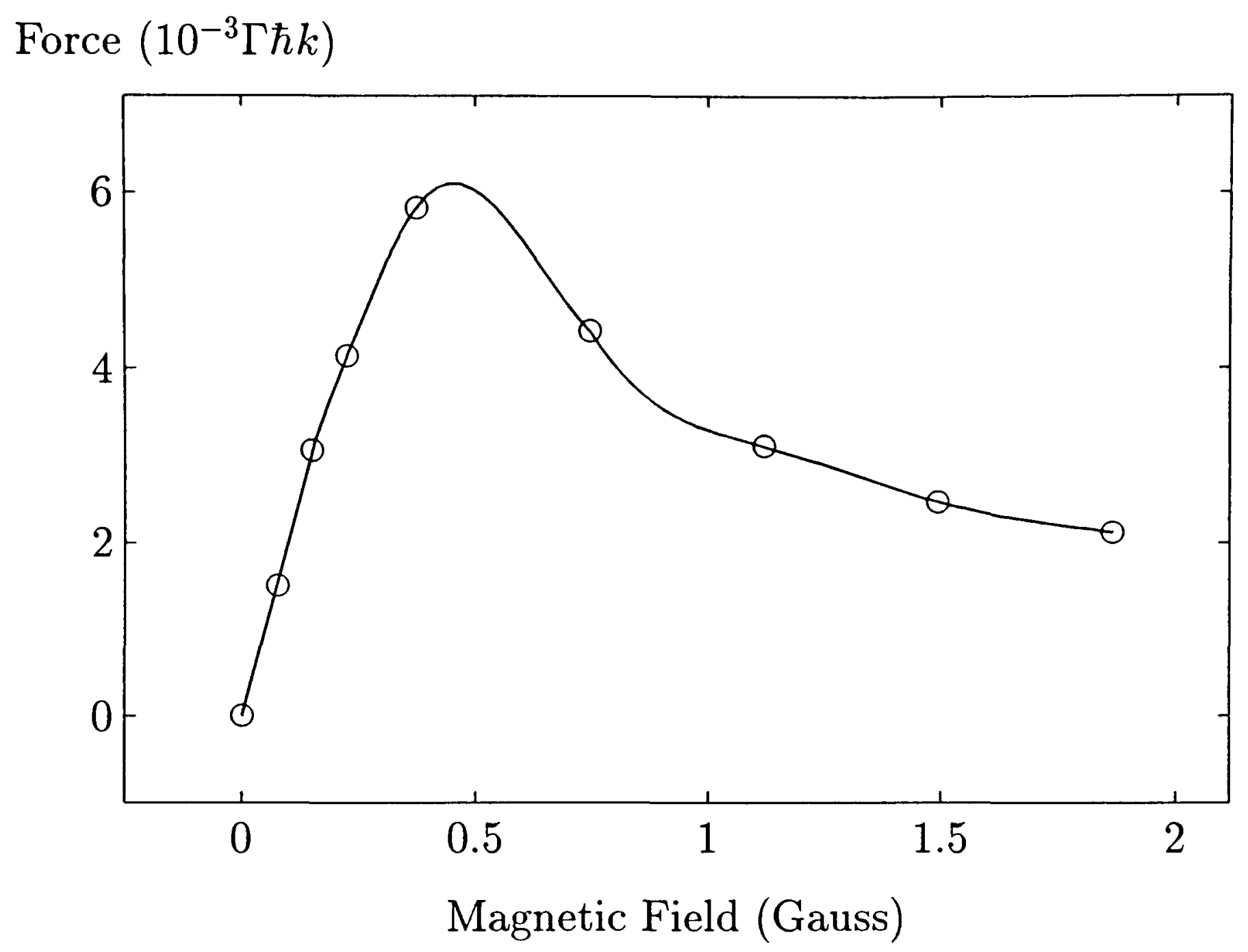

Figure 2.1: Force on a stationary atom in a MOT, as given by a semi-classical numerical threedimensional calculation [62]. The points are calculated, and the line is a cubic spline fit to guide the eye. The calculation used $\delta=6 \Gamma, \Omega=0.9 \Gamma$, and was performed for a $J=4 \rightarrow 5^{\prime}$ transition, with magnetic field scaled for a ground state Landé factor $g_{F}=1 / 4$. The figure illustrates the general form of the variation of the force with magnetic field.

same behaviour in three dimensions [62]. The variation of the force with magnetic field from this calculation is shown in Fig. 2.1 for a $J=4 \rightarrow 5^{\prime}$ transition and with $g_{F}=1 / 4$ for the ground state, as appropriate for caesium. Defining $r_{l}$ to be the radius at which the restoring force reaches a local maximum, the calculation yields the condition

$$
\mu_{B} b r_{l}=C_{l} \frac{\hbar \Omega^{2}}{\delta}
$$

for the boundary between the two regimes, where the constant $C_{l}$ is approximately 0.9 for the conditions just described. The calculation assumes the same Landé factor in the excited state as the ground state which is not appropriate for caesium, but at low saturation the result is not expected to be sensitive to this. Petrich et al. [68] have recently reported two-component density regimes at high magnetic field gradients and high detunings. It will be shown that Eq. (2.19) reproduces the onset of the two-component behaviour in their data as well as in our own. 


\subsubsection{Regime boundaries}

We now bring together the equations describing the different regimes of trap behaviour. The boundary between the temperature-limited and multiple scattering regimes is defined by $r_{T}=r_{\mathrm{MS}}$. Using Eqs. (2.5) and (2.18) this condition may be expressed in terms of the number of atoms trapped when the cloud just reaches this boundary:

$$
N=n_{\mathrm{MS}}\left(\frac{2 \pi k_{B} T}{\kappa}\right)^{3 / 2}
$$

Making use of Eq. (2.8) and the scaling with field gradient of Eq. (2.17) the above equation can be rearranged to find the variation of field gradient with number along the boundary:

$$
\frac{b}{b_{0}}=\frac{\delta}{\Gamma} \frac{1}{\kappa_{0}}\left(\frac{n_{\mathrm{MS}}}{\kappa}\right)^{2}\left(2 \pi k_{B} T\right)^{3} \frac{1}{N^{2}} .
$$

Figures 2.2(a)-(d) show "phase diagrams" for the MOT in the magnetic field gradient - number of trapped atoms plane, under different conditions of detuning and intensity that are readily accessible by experiment. Eq. (2.21) marks the predicted boundary between the temperature-limited and multiple scattering regimes and is indicated by solid line. Note that the prediction is really only valid for $\delta \gtrsim 4 \Gamma$ and $0.05 \Gamma \lesssim \Omega^{2} / \delta \Gamma \lesssim$ $0.4 \Gamma$ because of the restrictions on using Eq. (2.9) to give the molasses temperature. The diagrams confirm that with only a small number of atoms $\left(\sim 10^{4}\right)$ the trap is expected to be in the temperature-limited regime for most values of detuning and field gradient, whilst for larger numbers $\left(\gtrsim 10^{6}\right)$ it should be mostly in the multiple scattering regime. The variation of Eq. (2.21) with detuning and intensity is

$$
\frac{b}{b_{0}} \propto\left(\Omega^{2}\right)^{5 / 2} \delta \frac{1}{N^{2}}
$$

It is interesting to notice that $r_{T}$ is much more sensitive to a variation in field gradient or intensity than $r_{\mathrm{MS}}$ [compare the dependencies of Eqs. (2.10) and (2.18)]. Thus the behaviour of the boundary as these parameters are changed is qualitatively understood by just considering the effect they have on $r_{T}$. For example, when the field gradient is increased $r_{T}$ shrinks and thus the condition $r_{T}=r_{\mathrm{MS}}$ requires a smaller number of atoms; hence the boundary in the $\mathrm{d} B / \mathrm{d} z-N$ plane has a negative gradient. As the intensity is increased $r_{T}$ also increases and so the boundary at fixed field gradient moves to a larger number of atoms. This variation is perhaps a little counter-intuitiative as one might expect a larger scattering rate to force the boundary to smaller $N$. By contrast the variation in boundary position with detuning is in the direction expected; however, the dependence is much weaker than for a corresponding change in intensity. This is essentially because the spring constant is independent of intensity.

The boundary between the multiple scattering and two component regimes is defined by $r_{\mathrm{MS}}=r_{l}$. In defining this boundary it is recognized that $r_{\mathrm{MS}}$ is not the true extent of the trapped cloud; when the condition is satisfied it is expected that about half of the atoms will be in the diffuse ball extending beyond $r_{l}$. Using the defining 

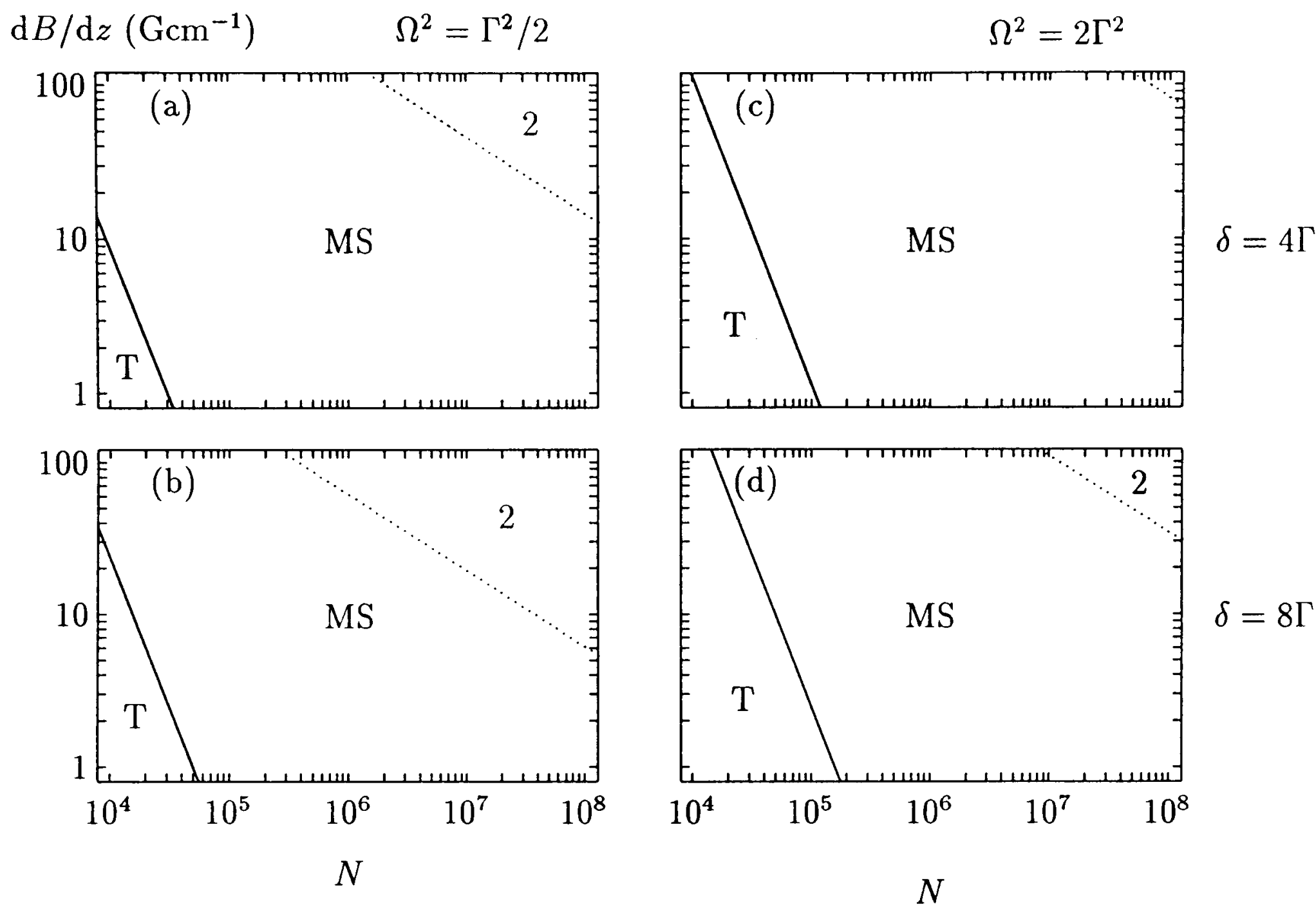

Figure 2.2: Different regimes of behaviour of a magneto-optical trap. The curves show the positions of the boundaries between the temperature limited, multiple scattering and two component regimes, marked T, MS and 2 respectively on the diagrams. Each diagram (a-d) is for a different combination of Rabi frequency and detuning, as follows: $\left\{\Omega^{2} / \Gamma^{2}, \delta / \Gamma\right\}=(\mathrm{a})\{0.5,4\}$, (b) $\{0.5,8\}$, (c) $\{2,4\}$, (d) $\{2,8\}$. The parameter values used are $\kappa_{0}=1 \times 10^{-19} \mathrm{Nm}^{-1}, C_{\mathrm{MS}}=50, C_{l}=1.4$ (taken from the measurements described in chapter 4 ).

Eqs. (2.18) and (2.19) we find that the number of trapped atoms when the cloud enters the two component regime is

$$
N=\left(\frac{\hbar \Gamma}{\mu_{B} b_{0}}\right)^{3} n_{\mathrm{MS}}\left(\sqrt{2 \pi} C_{l}\right)^{3}\left(\frac{\Omega^{2}}{\delta \Gamma} \frac{b_{0}}{b}\right)^{3} .
$$

Again this may be rearranged to find a formula for the field gradient. The scaling is

$$
\frac{b}{b_{0}} \propto\left(\frac{\Omega^{2}}{\delta \Gamma}\right)^{5 / 2} \frac{1}{\sqrt{N}}
$$

So this time the boundary position varies as a power of the light shift. The full dependence of Eq. 2.24 is represented by the dotted lines in Fig. 2.2.

Finally, the boundary $r_{T}=r_{l}$ between the temperature-limited and two-component 
regimes (which becomes important at low $\Omega$, high $\delta$ ) is given by

$$
\frac{b}{b_{0}}=\left(\frac{\hbar \Gamma}{\mu_{B} b_{0}}\right)^{2} \frac{\Omega^{4}}{\delta^{3} \Gamma} \frac{C_{l}^{2} \kappa_{0}}{k_{B} T}
$$

Under none of the conditions of Fig. 2.2 is the trap predicted to pass straight from the temperature-limited to two-component regime.

Finally we should remark again that a trapped cloud of atoms is not a thermodynamic system and thus the talk of regime boundaries and phase diagrams should be considered in a very loose way. In particular the boundaries indicated in Fig. 2.2 should not be expected to represent sharp transitions. For example, when the cloud begins to spill over into the two-component regime the confining force is weakened for some fraction of the atoms so that, when averaged across the whole cloud, the average spring constant is smaller than the value given by Eq. (2.8). Thus the average confinement is slightly weaker than the model predicts and this will hasten the onset of the two-component transition. Experimentally the changes between different regimes were indeed observed to be smooth.

\subsubsection{Optically thick regime}

For completeness we now outline the behaviour when the trap becomes optically thick. Sesko, Walker and Wieman [75] noticed that with large numbers of atoms $\left(N \gtrsim 5 \times 10^{7}\right)$ at high saturation of the atomic transition (saturation parameter per beam $s \sim 5$ ) the radius of the trap began to increase more rapidly with number than the $N^{1 / 3}$ dependence predicted by Eq. (2.18). Correspondingly the density was observed to decrease with number. Linquist, Stephens and Wieman [56] observed very similar behaviour and offered the following explanation. If the optical depth of the trap becomes large then there is eventually a significant probability that a trapping photon is scattered more than twice. In this instance the repulsive forces from radiation trapping are increased over those in the simple multiple scattering regime, and the cloud will tend to expand. However, expansion reduces the optical depth and thus also the repulsive forces, therefore tending to suppress expansion. An equilibrium is maintained such that the optical depth of the trap roughly remains a constant (presumably of order unity) as the number of atoms increases. Thus at fixed detuning and intensity

$$
\begin{aligned}
n_{\mathrm{OT}} r & \approx \text { constant } \\
\Rightarrow \frac{N}{r^{3}} r & \sim \text { constant } \\
\Rightarrow r & \propto N^{1 / 2} \\
\Rightarrow n_{\mathrm{OT}} & \propto N^{-1 / 2}
\end{aligned}
$$

Linquist, Stephens and Wieman [56] found that the variation of radius with number was indeed well described by a $N^{1 / 2}$ dependence.

The optically thick regime has not been of great importance in our experimental work. We have generally only been interested in the behaviour of the MOT under 
Table 2.2: The various regimes of the MOT. The radii $r_{T}, r_{\mathrm{MS}}$ and $r_{l}$ are defined in Eqs. (2.10), (2.18) and (2.19) respectively.

\begin{tabular}{lll}
\hline \hline Small $N$ & $r_{\mathrm{MS}}<r_{T}<r_{l}$ & $n \propto N, r$ independent of $N$ \\
Medium $N$ & $r_{T}<r_{\mathrm{MS}}<r_{l}$ & $n$ independent of $N, r \propto N^{1 / 3}$ \\
Large $N$ & $r_{l}<$ greater of $\left\{r_{T}, r_{\mathrm{MS}}\right\}$ & two-component density distribution \\
\hline \hline
\end{tabular}

conditions optimizing the sub-Doppler processes, i.e. at low saturation. However, it has been noticed on numerous occasions that the MOT density under loading conditions does. indeed vary with number, although no systematic study has been undertaken.

\subsubsection{Summary of the static MOT}

The three regimes that were relevant to our work are summarized in Table 2.2 and examples of their boundaries are illustrated in Fig. 2.2. The regimes can be explained in terms of the description of the MOT that has thus far been presented. The temperature and confinement at the centre of the trap are determined by polarization gradient forces and by multiple scattering of photons between atoms. Normally multiple scattering also limits the density, which is then almost independent of number. Under special circumstances the density can be determined by the number of atoms and by their temperature, but this is rare. The spatial distribution of the atoms changes when the trap passes from the temperature-limited to multiple scattering regimes, but experimentally we have observed that the cloud departs from its Gaussian shape to become uniform only well after the density has become independent of number (i.e. at high saturation and with large numbers of atoms). In other words, a Gaussian profile is not by itself a sign that the cloud is temperature-limited. Multiple scattering of photons also raises the temperature, the excess heating being proportional to $N^{1 / 3}$. A combination of high magnetic field gradient and small light shift, or else a sufficiently high number of trapped atoms, will cause the trapped cloud to spill outside the central stronglyconfining region of the MOT, leading to a two component density distribution and the eventual disappearance of sub-Doppler temperatures. From the derivation of the equations for Fig. 2.2 (Sec. 2.2.4) it is clear that the behaviour of the MOT is specified by four parameters: the detuning $\delta$ and Rabi frequency $\Omega$ of the trapping laser, the magnetic field gradient $b$, and the number of trapped atoms $N . \Omega$ and $\delta$ are perhaps more usefully parameterized by the light shift $\Omega^{2} / \delta \Gamma$ than the scattering rate since the former appears in the formulae for the temperature [Eq. (2.9)], the semiempirical density [Eq. (2.17)] and the onset of the two-component regime [Eq. (2.19)].

\subsubsection{Limitations of the model}

With regard to the MOT behaviour that has been observed, the model presented is limited in two respects. First, a detailed prediction for the behaviour of the density in the multiple scattering regime has not been incorporated. The model proposed by Walker, Sesko and Wieman was found to give a poor representation of the observed 
trends, but this has been replaced only by a semiempirical formula. Nevertheless, it will be possible to comment on the possibilities of various processes not included in the Walker, Sesko, Wieman model (such as spatial confinement of atoms in optical potential wells and the degradation of the spring constant by multiple scattering) being the cause of the discrepancy. The second limitation is that the effects of the breakdown of the sub-Doppler mechanism at low light shifts, in particular the weakening of the spring constant, has not been included. This breakdown will be considered further when the experimental results are discussed.

\subsection{The time-dependent MOT}

In practice it is generally not possible to study high phase-space densities in a steadystate MOT. This is because the processes determining the total number of trapped atoms are different from those determining the density and temperature, and the conditions under which the number is optimized do not yield low temperatures and high densities. From the description of the MOT presented thus far, it is clear that the properties of trapped atoms near the magnetic field zero are determined by polarization gradient forces, and that these work efficiently at low saturation of the atomic transition. By contrast, the forces which slow atoms from the background vapour sufficiently to allow their eventual capture (by the sub-Doppler mechanisms) are Doppler only, and are effective over the entire intersection volume of the trapping beams. The slowing process is efficient at high saturation of the atomic transition. Therefore, to observe MOT behaviour at high phase-space densities it is necessary to adopt the following transient procedure. The trap is first loaded with trapping parameters $b_{1}, \Omega_{1}, \delta_{1}$ chosen for an optimum steady-state number of atoms, $N_{1}$, but is subsequently switched rapidly to new values $b_{2}, \Omega_{2}, \delta_{2}$ which, according to the steady-state model of the MOT, lead to higher densities and lower temperatures. In doing this one must be clear under what circumstances the previous analysis of steady-state operation still applies because when the parameters are switched the trapped number decays to a new value $N_{2}$, which is much smaller than $N_{1}$ because the conditions are no longer optimal for trapping large numbers.

The suitability of the steady-state model is argued qualitatively in the following way. The time scale for an atomic density to relax to a new equilibrium shape is of order $\tau_{\text {pos }} / 3$, where $\tau_{\text {pos }}$ is the position damping time, i.e. the relaxation time for an atom displaced from the trap centre to move back towards the origin. With the assumption of spherical symmetry, $\tau_{\text {pos }}=\alpha / \kappa$, and is $\sim 30 \mathrm{~ms}$ for a typical magnetic field gradient (measurements of the position damping time are presented in Sec. 4.7). Now if the number of atoms does not decay appreciably during this interval then the previous analysis is valid. In other words, we can consider the state of the trap to adiabatically adjust to a steady-state operation with parameter $N=N(t)$. On the other hand, if the decay time of the trap is short enough to be comparable with the position relaxation time then the decay itself will affect the compression. Indeed the final density attained will clearly be reduced because the loss of atoms depletes the density on the same time scale as the position relaxation attempts to compress it. 
The preceding argument is quantified in the following way. The decay of $N(t)$ is determined by two different types of collision processes. They are mentioned briefly here in order to assess their potential for limiting the density, but are described more fully in the following section. The first is the lifetime associated with collisions with the room temperature background vapour. This lifetime is typically $\sim 1 \mathrm{~s}$ under the conditions optimal for loading the trap and with background pressures $\sim 10^{-8}$ Torr, as we used in our experiments. It becomes shorter when the trapping conditions are switched to those of low saturation (since the trap is weaker), but generally does not get close to encroaching upon the position relaxation time. It may thus be safely assumed that this collision process cannot affect the density. The second type of collision process is that which occurs between trapped atoms. These cold collisions induce trap loss at a rate $\beta n(\mathbf{r})$ per atom, where $\beta$ is the cold collision loss coefficient [70], and their time scale for collisions is characterized by $1 / \beta n$. Under certain circumstances we have measured $\beta$ to exceed $10^{-10} \mathrm{~cm}^{3} \mathrm{~s}^{-1}$ (chapter 5); yet for densities not larger than a few $10^{11} \mathrm{~cm}^{-3}$ (as were the largest we observed) this second time scale is also much larger than the position relaxation time. We conclude, therefore, that to a good approximation the time-dependent MOT may be considered as a quasistatic system, described at each time $t$ by a steady-state model.

It is nevertheless instructive to formulate an expression for a collision-limited density. This is found by equating the rate at which the density increases due to position relaxation to the rate at which it decreases due to loss of number. The former is simply $3 n / \tau_{\text {pos }}$. The latter is more difficult to guess at but is found from the equation of motion for the density. We derive this equation from the decay of the trap number. Making the simplifying assumptions that cold collisions dominate the trap loss, that the spatial density is uniform, and that $N_{2} \ll N_{1}$ (i.e. when the conditions are switched the loading rate, $R$, becomes negligible) we have

$$
\frac{\mathrm{d} N}{\mathrm{~d} t} \approx-\beta n N \text {. }
$$

Differentiating $N=n V$ with respect to time and combining the resulting expression with Eq. (2.29) divided by $V$ gives the equation of motion

$$
\frac{\mathrm{d} n}{\mathrm{~d} t}=-\beta n^{2}-n \frac{1}{V} \frac{\mathrm{d} V}{\mathrm{~d} t}
$$

By putting $V \propto r^{3} \propto e^{-3 t / \tau_{\text {pos }}}$ we recognize that the second term on the right-hand side is due to the position relaxation:

$$
\dot{n}=-\beta n^{2}+\frac{3 n}{\tau_{p o s}} .
$$

In the steady-state $\dot{n}=0$ which implies a collision-limited density $n_{\mathrm{CL}}=3 / \beta \tau_{\text {pos }}$. In practice there is always an additional $n^{2}$ term on the right-hand side of Eq. (2.31) to account for the density tending towards a multiple scattering-limited density in the absence of collisions. We thus conclude that

$$
n \leqslant n_{\mathrm{CL}}=\frac{3}{\beta \tau_{\text {pos }}}=\frac{3 \kappa}{\alpha \beta}
$$


where the last step assumes spherical symmetry. We note in passing that Eq. (2.30) reveals that the density decreases at a rate $-\beta n^{2}$ due to cold collisional loss. If the volume $V$ is constant with time (i.e. constant with number, as in the temperaturelimited regime) then this result is obvious from Eq. (2.29). In various articles the constant volume result has been quoted and subsequently applied to the general case of a volume varying with time. The justification in doing this has been demonstrated above.

\subsection{Collisional processes}

The rate equation (1.9) describing the number of atoms in the trap is more instructively written in the following way:

$$
\frac{\mathrm{d} N}{\mathrm{~d} t}=R-\frac{N}{\tau}-\beta \int n^{2}(\mathbf{r}, N) \mathrm{d}^{3} \mathbf{r}
$$

where $R$ is the loading rate described previously. The trap loss has now been separated into two contributions. The first term represents collisions with the room temperature background vapour at a rate $\tau^{-1}$ per atom. The second accounts for losses due to cold collisions between the trapped atoms and depends on a rate coefficient $\beta$ [70] and the square of the spatial density.

The clearest manifestation of cold collisions is the trap loss represented by the beta term above, and measuring this rate has been the basis of nearly all experiments designed to study cold collision phenomena to-date. The measurement is usually made by loading a small number of atoms into the trap from an atomic beam, and then shuttering the beam and observing the subsequent trap decay. In the temperaturelimited regime the trap dimensions remain constant as the number decreases and thus the decay due to cold collisions is distinguished from that due to collisions with the background vapour by its $N^{2}$ dependence.

Since the density is a parametric function of the trap number $N$, the transient solution to Eq. (2.33) is different in the temperature-limited and multiple scattering density regimes. However, when loading from a vapour the trap passes into the latter typically after only a small fraction of the final trapped number has been captured so that, for all but the very shortest times, the density is roughly uniform and virtually independent of number. It can then be taken outside the integral and solving Eq. (2.33) then yields the following loading curve:

$$
\begin{aligned}
N(t) \approx N_{\infty}\left(1-e^{-\Gamma t}\right) & \text { where } \Gamma \equiv \tau^{-1}+\beta n \\
& \text { and } N_{\infty} \equiv R \Gamma^{-1}
\end{aligned}
$$

Measuring the loading time $\Gamma^{-1}$ has been the basis of a recent method to determine $\beta$ [41].

A vapour cell arrangement will load between $10^{7}$ and $10^{8}$ atoms when the background vapour pressure is $\sim 10^{-8}$ Torr, with trapping beams diameters $\sim 10 \mathrm{~mm}$, and 
with trapping conditions optimal for loading. At this pressure the lifetime against background collisions is $\tau \sim 1 \mathrm{~s}$. Under these trapping conditions the MOT density is typically $n \sim 10^{10} \mathrm{~cm}^{-3}$ (chapter 4 ) and $\beta$ is typically $\sim 2 \times 10^{-11} \mathrm{~cm}^{3} \mathrm{~s}^{-1}$ (chapter 5). Thus the lifetime against cold collisions is $1 / \beta n \sim 5 \mathrm{~s}$, which is a lot longer than $\tau$ and is therefore generally not noticeable. Consequently traditional methods to study $\beta$ have resorted either to substantially increasing $\tau$ (to in excess of $100 \mathrm{~s}$ ) by use of ultra-high vacuum systems $[70,74,87]$, or to artificially increasing $\beta$ by means of an additional light field $[74,42,41,58]$. In chapter 5 it is demonstrated that under certain conditions the loss effects from cold collisions can be observed without the use of either of these techniques.

\subsubsection{Collisions with the background vapour}

Under typical loading conditions the escape velocity from the MOT is of order $10 \mathrm{~ms}^{-1}$, corresponding to a trap depth of about $1 \mathrm{~K}$. By comparison, the background vapour is at room temperature and thus a background atom-trapped atom collision has the possibility to transfer sufficient energy to the trapped atom to cause its ejection from the trap. For a background vapour of density $n_{b}$, whose atoms are travelling with a mean speed $\bar{c}$ much greater than those of the trapped atoms, such collisions occur at a rate

$$
\frac{1}{\tau}=n_{b} \sigma_{e s c} \bar{c}=\frac{\sigma_{e s c} P_{b}}{\sqrt{\pi m k_{B} T_{b} / 8}}
$$

where $P_{b}$ and $T_{b}$ are respectively the pressure and temperature of the background vapour and $\sigma_{\text {esc }}$ is the collision cross-section leading to trap loss.

It is useful to have some idea of how $\tau$ varies with the escape velocity, $v_{e s c}$. The steps involved in a simple estimate of this dependence are now outlined. First, we note that for $\tau \sim 1 \mathrm{~s}$ at $P_{b} \sim 10^{-8}$ Torr, Eq. (2.35) gives $\sigma_{e s c} \approx 14 \times 10^{-14} \mathrm{~cm}^{2}$. This implies that the collision duration, which might be thought of roughly as $\sqrt{\sigma_{e s c}} / \bar{c}$, is of the order of $10 \mathrm{ps}$. This time is much shorter than the $32 \mathrm{~ns}$ lifetime of the $6 \mathrm{P}_{3 / 2}$ excited level in caesium and thus means that absorption and emission effects can be neglected in a background collision calculation. A second step is to appreciate that although a velocity transfer to the trapped atom of close to the escape velocity is small compared to the relative velocity of the colliding atoms, it is still large enough to be able to describe the scattering classically. That is, we are not dealing with scattering close enough to the forward direction to warrant a consideration of interference effects. Justification for this is given later when, for a different calculation, velocity transfers much smaller than $v_{e s c}$ are considered. A purely classical calculation greatly simplifies the method which can then proceed in a similar vein to that outlined in [77]. There the interaction potential was taken to comprise of only the longest range, spherically symmetric interaction:

$$
V(r)=\frac{C_{n}}{r^{n}}
$$

and the trajectory of the background atom was assumed to be unaffected by the finite velocity acquired by the trapped atom. This yielded the following expression for the 
required cross-section:

$$
\sigma_{e s c}^{n}=\pi\left(\frac{f(n) C_{n}}{m v_{e s c} \bar{c}}\right)^{2 / n}
$$

where $n$ is the order of the interaction: 3 for resonant dipole-dipole and 6 for van der Waals, and $f(3)=4, f(6) \approx 5.9$. The interaction constants for the $6 \mathrm{~S}_{1 / 2}-6 \mathrm{P}_{3 / 2}$ transition in caesium are

$$
\begin{aligned}
C_{3}=\frac{d^{2}}{4 \pi \epsilon_{0}} & =\frac{3}{4}\left(\frac{\lambda}{2 \pi}\right)^{3} \hbar \Gamma \\
& =6.56 \times 10^{-48} \mathrm{Jm}^{3}[77] \\
\text { and } C_{6} & =6.95 \times 10^{-76} \mathrm{Jm}^{6}[47] .
\end{aligned}
$$

The actual cross-section is obtained by summing $\sigma_{\text {esc }}^{3}$ and $\sigma_{e s c}^{6}$, weighting each term fractionally by the proportion of atoms in the ground and excited states.

Several features of Eq. (2.37) are worth noting. First, it gives good quantitative agreement with observed escape cross-sections: taking the excited state fraction to be 0.3 (typical for loading conditions) and $v_{e s c}$ to be $15 \mathrm{~ms}^{-1}$ gives $\sigma_{\text {esc }} \approx 14.1 \times$ $10^{-14} \mathrm{~cm}^{2}$. Secondly, because it is often the case that the majority of trapped atoms are in the ground state (for example when the scattering rate is lowered to produce low temperatures or high densities) and, therefore, interact mainly through the van der Waals force, the prediction is a lifetime against background collisions that varies only slowly with escape velocity: $\tau \propto v_{e s c}^{1 / 3}$. Finally, it is interesting to compare the magnitude of the cross-section predicted by Eq. (2.37) with that of the total collision cross-section. The total cross-section must be calculated quantum mechanically rather than classically because the effects of forward scattering must be considered. It is given on page 1324 of reference [59] in the limit of many partial waves (i.e. large relative velocity of the colliding atoms):

$$
\sigma_{\text {tot }}=g(n)\left(\frac{C_{n}}{\hbar \bar{c}}\right)^{2 /(n-1)}
$$

where $g(3) \approx 20.2$ and $g(6) \approx 8.1$. When substituted with the relevant constants for caesium this gives $\sigma_{t o t} \approx 200 \sigma_{e s c}$ for $n=3$ and $\sigma_{t o t} \approx 5 \sigma_{e s c}$ for $n=6$. In other words, only a small fraction of the trapped atom-background vapour collisions actually cause trap loss.

In the light of this conclusion it is interesting to estimate the significance of the heating effect from those glancing collisions not contributing to loss. The quantity of interest is the mean heating rate per collision which, when combined with the temperature damping time in the MOT, gives a collision-induced temperature. This can then be compared to typical MOT temperatures to gauge its significance.

We first estimate the mean square velocity transferred to a trapped atom by a collision not inducing loss. This is given by

$$
\overline{v^{2}}=\int_{0}^{v_{e s c}} v^{2} \sigma(v) \mathrm{d} v /\left(\sigma_{t o t}-\sigma_{e s c}\right)
$$


where $\sigma(v)$ is the differential cross-section for velocity transfers between $v$ and $v+$ $\mathrm{d} v$. The use of a classical expression for the cross-section in this instance requires more justification because the integral (2.42) explicitly deals with very small scattering angles. The classical approach always fails at some small deflection angle $\theta_{c l}$ because the de-Broglie wavelength of the momentum transferred becomes comparable to the interaction distance, and interference effects are then important. Thus if $\sqrt{\sigma_{\text {tot }} / \pi}$ is taken as a measure of the interaction distance,

$$
\frac{2 \pi}{k_{\text {rel }} \theta_{c l}} \sim \sqrt{\frac{\sigma_{t o t}}{\pi}}
$$

where $k_{r e l}$ is the relative wavevector of the colliding atoms. In particular for an interaction of the form (2.36) the classical cross-section becomes infinite as the scattering angle tends to zero, and this is verified by the form of Eq. (2.37). By contrast, the quantum mechanical cross-section is always finite in the forward scattering direction ${ }^{1}$. To estimate $\overline{v^{2}}$, however, the classical form for $\sigma(v)$ will be used with the justification that in the integral (2.42) the small scattering angles (small $v$ ) are given zero weight at a rate faster than the classical expression for $\sigma(v)$ becomes divergent. This amounts to saying that the mean deflection $\bar{\theta} \approx \sqrt{v^{2}} / \bar{c}$ resulting from evaluation of the integral is much larger than $\theta_{c l}$. For example, for $n=3$ Eqs. (2.41) and (2.43) give $\theta_{c l} \sim 10^{-4}$ radians, whereas for the values of $v_{e s c}$ we consider $\bar{\theta}$ will turn out to be $\sim 10^{-3}$ radians.

So having justified a classical approach, the calculation is again straightforward. $\sigma(v)$ is obtained by replacing $v_{\text {esc }}$ by $v$ in Eq. (2.37) and differentiating with respect to $v$. Evaluation of the integral (2.42) with this expression then gives

$$
\overline{v^{2}}=v_{e s c}^{2} \times \frac{\sigma_{e s c}}{\left(\sigma_{t o t}-\sigma_{e s c}\right)} \times \frac{1}{(n-1)} .
$$

We note that since $\sigma_{t o t} \gg \sigma_{e s c}$ and since $v_{e s c}$ corresponds to a temperature $\sim 1 \mathrm{~K}$ under loading conditions, this equation predicts an average heating per collision on the scale of $1 / 200 \times 1 / 2 \mathrm{~K}=2.5 \mathrm{mK}$ for the resonant dipole-dipole interaction. Equation (2.44) is converted into a rate by dividing by the time between collisions; we write this time as $\tau_{\text {tot }}$ because the relevant cross-section for its evaluation is $\sigma_{t o t}-\sigma_{e s c} \approx \sigma_{\text {tot }}$. If one makes the reasonable assumption that the heating is isotropic, then the excess temperature $\Delta T$ is $(3 / 2) k_{B} \Delta T=(1 / 2) m \overline{v^{2}}$, which leads to

$$
R_{T}=\frac{\mathrm{d} T}{\mathrm{~d} t}=\frac{m v_{e s c}^{2}}{3 k_{B}(n-1) \tau_{t o t}} \times \frac{\sigma_{e s c}}{\left(\sigma_{t o t}-\sigma_{e s c}\right)} .
$$

The significance of this heating is now examined. Consider typical loading conditions. With $v_{e s c}=15 \mathrm{~ms}^{-1}$ and a background pressure of $10^{-8}$ Torr Eq. (2.45) predicts $R_{T} \approx 1.5 \mathrm{Ks}^{-1}$ for $n=3$ and $R_{T} \approx 0.1 \mathrm{Ks}^{-1}$ for $n=6$, where use has been made of Eq. (2.37) to evaluate $\tau_{\text {tot }}$. With a fraction 0.3 in the excited level this corresponds

\footnotetext{
${ }^{1}$ except for the special case of $n=1$ (Rutherford scattering) where the quantal cross-section is also divergent (and identical to the classical result).
} 
to a net heating rate of approximately $0.5 \mathrm{Ks}^{-1}$. Now if $\tau_{\alpha}$ is the MOT temperature damping time then the excess temperature this heating rate induces is $\Delta T=R_{T} \tau_{\alpha}$. Recent measurements ([25] and chapter 5) suggest that $\tau_{\alpha}$ lies on a timescale of $50 \mu \mathrm{s}$ thus implying that $\Delta T \sim 25 \mu \mathrm{K}$. This is much smaller than the $100-200 \mu \mathrm{K}$ MOT temperatures under typical loading conditions, and thus, as might have been expected, the heating goes unnoticed. A similar conclusion is reached for conditions of low light shift and large detunings at which the lowest MOT temperatures of $2-3 \mu \mathrm{K}$ ([25], chapter 4) are attained: a prediction of $\Delta T \sim 10^{-2} \mu \mathrm{K}$ is obtained with $n=6$ (i.e. very small excited state population), and when it assumed that tau is reduced at these low scattering rates from its value under loading conditions by some factor $\sim 3$ (this is reasonable from measurements to be presented later), and that $\tau_{\alpha}$ is relatively insensitive to a change of scattering conditions [25].

To conclude, the excess heating from glancing collisions is effectively dissipated in the MOT by the friction damping forces. By comparison in a purely magnetic trap there is no damping and heating from such collisions has been observed [65]. Unfortunately for a magnetic trap the estimate given above is not applicable; trap depths are orders of magnitude smaller than those of the MOT, and the condition $\bar{\theta} \gg \theta_{c l}$ for the justification of a classical approach is no longer satisfied. This then is rather an unusual example of a high energy collision (involving many partial waves) which is untreatable classically.

\subsubsection{Cold collisions}

\subsubsection{Introduction}

Since the earliest days of optical trapping there has been much interest in the intratrap collision processes occurring at ultracold temperatures. An original motivation was that trapped atoms might provide an atomic source free from the line broadening and shifting associated with motional and collisional effects at higher temperatures. However, it was immediately apparent from the first measurement of a sub-millikelvin collision process [35] that unusual collisional effects are dominant at ultracold temperatures and that these give rise to large collision cross-sections. For two reasons the field of ultracold collisions has developed extremely rapidly. One has been the opportunity to investigate new fundamental physics, which is not apparent at higher temperatures. The other has been the practical reason that collisions are significant for attempts to observe collective quantum effects in ultracold vapours. Here there has been the incentive that understanding inelastic collision processes might lead to ways of minimising two-body trap loss, which was shown in Sec. 2.3 to ultimately limit the density of an optically trapped sample. Furthermore, the nature of elastic collisions is believed to play an important role in the formation of a stable Bose-Einstein condensate $[46,80]$.

The novel features of low energy collisions in a laser field are several and arise from the large de Broglie wavelengths and the influence of the radiation field on the collision dynamics of slow-moving atoms. The effects are understood qualitatively as follows. First there are interesting properties inherent to low temperatures. As $T \rightarrow 0$ the collision rate of an inelastic process becomes constant but the relative velocity of 
the colliding atoms becomes vanishingly small. This leads to a divergent cross-section. Furthermore, at the temperatures typically encountered in a MOT it is common for cross-sections to involve only a few partial waves. That is, $k_{B} T$ is so small that only the lowest angular momentum states can be sustained in a collision. Additionally, at some characteristic temperature the de Broglie wavelength becomes comparable to the spatial scale of the interaction, and there is then an onset of quantum behaviour. It is then inappropriate to treat the collision semiclassically. The second important feature is the effect of the radiation field on the interatomic potential. In the absence of radiative effects the longest range interaction is the $1 / r^{5}$ quadrupole-quadrupole potential for two excited atoms, or the $1 / r^{6}$ van der Waals potential for ground state atoms. In an optical field, however, the resonant dipole-dipole interaction between ground and excited state atoms induces a much longer $1 / r^{3}$ interaction. The effect of this interaction depends on the atom separation. At large separations, where the atoms interact mainly with the field rather than with each other, the influence of the dipole-dipole term on an intense field is to induce a $1 / r^{3}$ dependence on the long-range potential [48]. For dressed states involving one excited state and one ground state atom, both attractive and repulsive curves can be excited, but the attractive curve is preferentially excited because the laser field is detuned to the red of the resonance. Thus the interaction acts to preferentially capture atoms into an optical potential. At small separations the collision dynamics for the attractive state is governed entirely by the dipole-dipole interaction, i.e. the atom is completely decoupled from the laser field. The transition between the coupled and uncoupled regimes occurs roughly at a distance where the dipole-dipole potential, $C_{3} / r^{3}$, is equal to the splitting between the dressed field states, $\sqrt{\delta^{2}+\Omega_{\text {tot }}^{2}}$. In a MOT the latter is typically of the order of a few linewidths, and it is thus seen from Eq. (2.38) that the dipole-dipole interaction is dominant over separations up to of order $\lambda / 2 \pi$. In the decoupled region the potential is much larger than $k_{B} T$ and thus there is a large effect on the collision dynamics. The third feature of low energy collisions is the novel role of spontaneous emission in the collision process involving a resonant dipole-dipole interaction. The long range of the potential combines with the small atomic velocities to give collision times so long that there is a significant probability of spontaneous decay during the collision itself. The effect of decay is to radically alter the collision dynamics, but more significantly to cause a large reduction in the collision rate. In conventional time-independent scattering theory there is no provision for a change in the total energy of the colliding pair, and it has been necessary to find new approaches to describe excited state collisions at ultracold temperatures.

With reference to Fig. 2.3 cold collisions in caesium can be classified into one of two distinct categories. In the first, termed ground state collisions, both atoms remain in their ground electronic states throughout. They are subject to conservative atomic forces only, as in a conventional collision, but because the van der Waals force is of short range the collisions must be treated quantum mechanically. There are both elastic and inelastic processes, and the cross-sections are extremely sensitive to the details of the interaction potential, typically involving only the first few partial waves. In the second type of collision, referred to as light-assisted collisions, one of the colliding atoms is resonantly excited to the $6 \mathrm{P}_{3 / 2}$ level by the trapping laser field whilst the other 


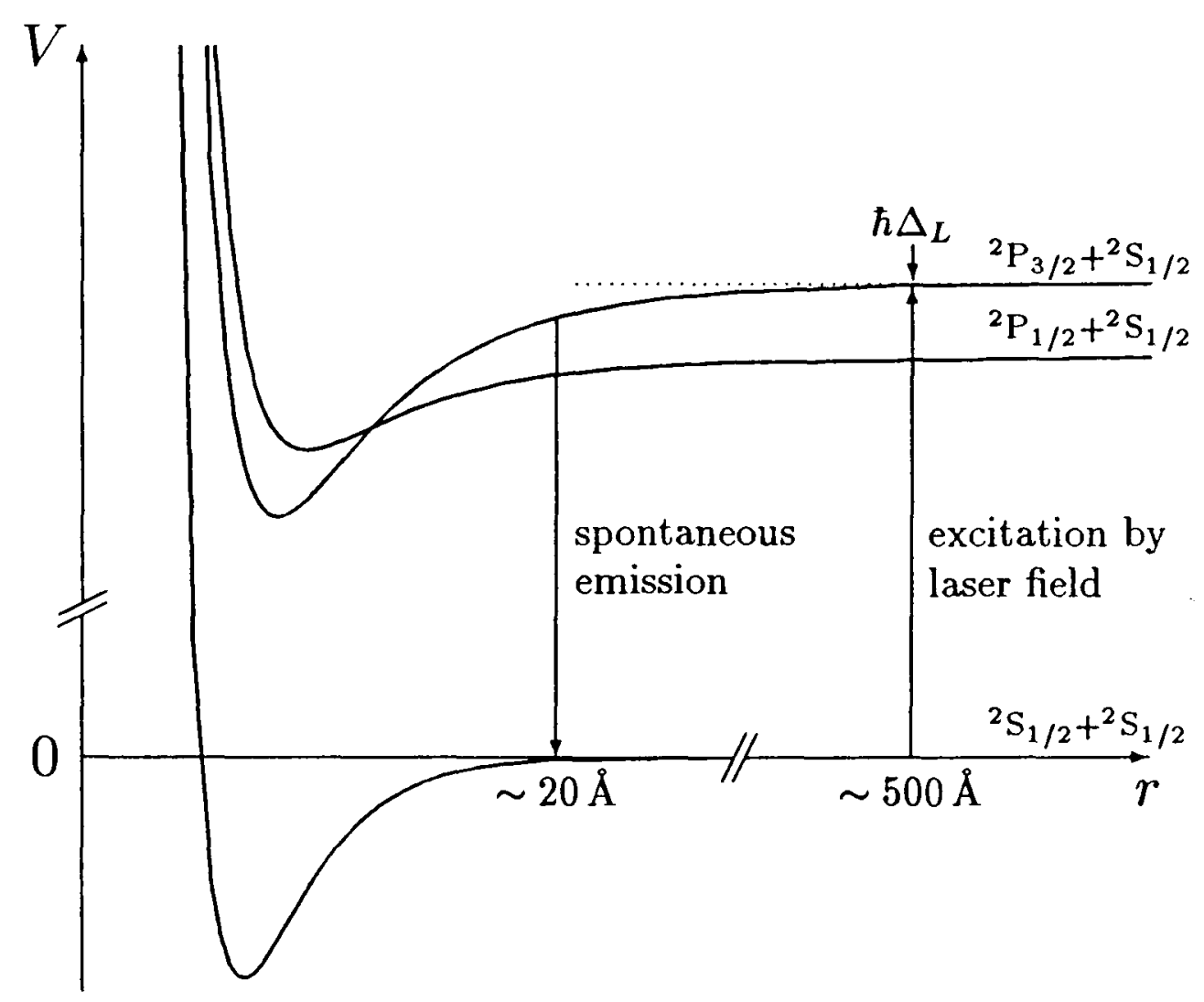

Figure 2.3: Schematic diagram of a light-assisted collision process. The molecular level $\mathrm{S}_{1 / 2}+\mathrm{P}_{3 / 2}$ is resonantly excited at large separation $r$ by the red detuned laser field. The atoms are accelerated towards each other by the $r^{-3}$ potential and sometime later either emit a red-shifted photon (as shown) or change fine-structure state. The $S_{1 / 2}+P_{3 / 2}$ and $S_{1 / 2}+S_{1 / 2}$ curves have been plotted using the appropriate values of $C_{3}$ and $C_{6}$ for caesium.

atom remains in the ground state. The atoms accelerate towards each other, rolling down the long-range resonant dipole-dipole potential. After some travel distance the collision terminates with either the emission of a red-shifted photon and consequently a gain in kinetic energy when the atoms have separated (radiative redistribution), or at sufficiently close distance by a change of state to the $6 \mathrm{~S}+6 \mathrm{P}_{1 / 2}$ level, with a correspondingly large gain in kinetic energy (fine structure-changing collision). In some of the other alkalis, notably sodium, there is a third category of collision involving both atoms in the excited $\mathrm{P}_{3 / 2}$ level. This can lead to associative ionisation (AI), a signature of which can be detection of the emitted electron.

\subsubsection{Groundstate collisions}

This type of collision encompasses both elastic and inelastic processes. Studies of the former have consisted of measuring frequency shifts in a laser-cooled atomic clock $[32,85,34]$ and the time for temperature thermalization in a magnetic trap $[65,23]$, whilst the hyperfine state-changing collision is the most important example of the latter and has been observed on several occasions [74, 42, 87] (see also chapter 5). In the MOT the energy transfers from elastic collisions are small compared to the heating and cooling effects of the trapping light field and consequently go unnoticed.

The nature of ground state collisions at low energies nicely illustrates the novel 
features of highly quantum mechanical cross-sections with few contributing partial waves. Consider the attractive potential $C_{n} / r^{n}$. For $n \geqslant 3$ there is always a maximum in the long-range potential caused by the rotational energy $\hbar^{2} l(l+1) / 2 \mu r^{2}$ of a relative angular momentum $\hbar l$ (where $\mu=m / 2$ is the reduced mass). This maximum occurs at a separation

$$
r_{\text {max }}(l)=\left(\frac{n \mu C_{n}}{\hbar^{2} l(l+1)}\right)^{1 /(n-2)}
$$

and is crucially important to cold collisions because at low energies it restricts the range of $l$ that contributes to the cross-section. Equating the collision kinetic energy $3 k_{B} T / 2$ to the height of this maximum yields an estimate of the maximum permitted $l$ at a temperature $T[47]$ :

$$
l_{\text {max }}\left(l_{\text {max }}+1\right)=\left(\frac{n \mu C_{n}}{\hbar^{2}}\right)\left[\frac{3 k_{B} T}{(n-2) C_{n}}\right]\left(\frac{n-2}{n}\right)
$$

Using Eq. (2.40) for the magnitude of the van der Waals interaction in caesium, this relation gives $l_{\text {max }}=2$ at the Doppler temperature $T_{D} \approx 125 \mu \mathrm{K}$. The corresponding value of $r_{\max }$ is about 150 Bohr radii which is much larger than the separation at which any energy exchange in a ground state collision can take place. The conclusion of this is that under normal trapping conditions the existence of the barrier blocks access to the region $r<r_{\max }$ for all but the lowest angular momentum partial waves in a ground state collision. This is a familiar idea in partial wave analysis: roughly speaking, a wave with angular momentum $l$ does not contribute to the cross-section if its classical impact parameter lies outside the interaction range, i.e. if $\lambda_{d B} l>r_{\max }$.

A related question is at what temperature is quantum threshold behaviour observed, i.e. when is $\lambda_{d B}$ comparable to the interaction distance? Taking the interaction distance to be that at which the energy shift is one linewidth (i.e. roughly the distance to the decoupled region) gives the temperature to be several hundred microkelvin for a caesium ground state collision. From the above angular momentum consideration this suggests that when quantum effects set in only a few partial waves contribute to the cross-section, and clearly as the temperature is decreased eventually only $s$ wave scattering $(l=0)$ will remain. It is possible to appreciate the existence of very large collision cross-sections at ultracold temperatures. The quantum mechanical crosssection may be conveniently formalised as [35]

$$
\begin{aligned}
\sigma(T) & =\left(\pi / k_{r e l}^{2}\right) \sum_{l=0}^{\infty}(2 l+1) P(T, l) \\
& \approx\left(\pi / k_{r e l}^{2}\right)\left(l_{\max }+1\right)^{2} \bar{P}\left(T, l_{\max }\right)
\end{aligned}
$$

where $P$ denotes the scattering probability for the partial wave $l$ (and is the quantum analogue of the classical differential cross-section $\sigma(T, l))$ and $\bar{P}$ its average over the different $l$ involved. A calculation of $P$ requires an accurate knowledge of the potentials involved and is complicated, but it can be shown that $P \leqslant 1$ for inelastic scattering and $P=4$ for elastic scattering (note the similarity of Eq. ??eq:aaphaseshift) give a familiar partial wave elastic scattering result). Assuming $s$-wave scattering only then 
at a temperature $T=100 \mu \mathrm{K},\left(\pi / k_{\text {rel }}^{2}\right)$ predicts a cross-section $\sigma$ of order $5 \times 10^{-13} \mathrm{~cm}^{2}$ in caesium. This is at least four orders of magnitude larger than the cross-sections measured at temperatures of $20 \mathrm{~K}$ and higher [35].

2.4.2.2.1 Elastic ground state processes. For evaporative cooling to provide many orders of magnitude of cooling the initial elastic scattering rate must vastly exceed any trap loss rates. Elastic scattering rates in a magnetic trap have been measured for caesium by Monroe et al. [65] and for sodium by Davis et al. [23]. By loading the trap asymmetrically and monitoring the time taken for the initially unequal temperatures in the three mutually orthogonal directions to equalize, the elastic scattering cross-section was deduced following a measurement of the density and an estimate of the average number of collisions per atom required for thermalization. Accurate calculations of the elastic cross-section are notoriously difficult because the theory is sensitive to the position of the highest energy bound state. This state lies close to zero energy in caesium, giving a large cross-section which is predicted to be sensitive to application of an external magnetic field [82]. The sign of the scattering length is important to the formation of a weakly interacting Bose condensate. Current theory predicts a stable condensate for a positive scattering length and with a large number of condensed atoms; for a negative scattering length a condensate is expected to contract, inducing large two- and three-body loss rates with the resulting increase in density [8]. Bradley et al. [7] have recently reported observation of a Bose condensate in ${ }^{7} \mathrm{Li}$, for which the scattering length is known accurately to be negative by two-photon photoassociation spectroscopy and analysis of ${ }^{7} \mathrm{Li}_{2}$ spectra.

2.4.2.2.2 Inelastic ground state processes. Hyperfine-state changing collisions are of importance in the MOT because they have the potential to induce large trap losses. Two ground state atoms collide with one or both ending up in the lower hyperfine level:

$$
\begin{aligned}
\mathrm{Cs}\left(F_{1}=4\right)+\mathrm{Cs}\left(F_{2}=4 \text { or } 3\right) & \rightarrow \mathrm{Cs}_{2} \rightarrow \mathrm{Cs}\left(F_{1}=3\right)+\mathrm{Cs}\left(F_{2}\right)+\Delta E_{h f s} \\
\operatorname{Cs}\left(F_{1}=4\right)+\operatorname{Cs}\left(F_{2}=4\right) & \rightarrow \mathrm{Cs}_{2} \rightarrow \operatorname{Cs}\left(F_{1}=3\right)+\operatorname{Cs}\left(F_{2}=3\right)+2 \Delta E_{h f s}
\end{aligned}
$$

The resulting gain in kinetic energy for the first event, $\Delta E_{h f s} / 2$ per atom, corresponds in caesium to a velocity of about $5 \mathrm{~ms}^{-1}$ or, equivalently, a temperature of about $0.2 \mathrm{~K}$. Thus for these processes to cause loss the trap must be weak: under loading conditions the trap depth is typically $\sim 2 \mathrm{~K}$ and the losses are consequently suppressed. Indeed, the first unambiguous report of trap loss due to ground state collisions [74] measured loss rates from a caesium MOT at very low trapping beam intensities. As the intensity was increased from the low starting value an initial rapid decrease in $\beta$ was observed, and this was attributed to the trap suddenly becoming deep enough to capture those atoms ejected from the hyperfine-changing collisions.

The same study further revealed the significance of the hyperfine-changing collision as a potential mechanism for limiting the maximum density attainable in a MOT. As the intensity was further increased the losses due to the hyperfine-changing collisions were completely suppressed and a minimum $\beta$ about a factor of 25 smaller than 
the starting value was observed. The losses then began to rise again with increasing intensity due to the contribution from light-assisted collisions, but only at a rate proportional to the trapping intensity. When the intensity was a factor six larger than its starting value $\beta$ was still an order of magnitude smaller than its initial value at the lowest intensities. The conclusion of this is that hyperfine-changing collisions typically give the largest loss rates and furthermore under circumstances where the highest densities are expected to be attained, i.e. at low scattering rates.

\subsubsection{Light-assisted collisions}

Collisions involving a single excited state have been the subject of much study, both theoretical and experimental, because of the interesting characteristic that their collision dynamics are determined by the long-range resonant dipole-dipole interaction and by spontaneous emission. Such collisions are important in the MOT because although the trap loss rates they induce are generally smaller that those of hyperfine-state changing collisions, they produce loss for all trap depths. At low background vapour pressures light-assisted collisions are typically the dominant source of trap loss, and are the factor limiting the total number [2]. Their suppression in a dark MOT (see chapter 6) was the key to the recent attainment of sub-microkelvin temperatures in rubidium by evaporative cooling $[69,1]$.

A useful insight into the nature of a singly-excited state collision is obtained by calculating the temperature for quantum threshold behaviour. An estimate of this is easily made for caesium by comparing the constants $C_{3}$ and $C_{6}$ from Eqs. (2.39) and (2.40). They reveal that the spatial range of the dipole-dipole potential is of the order of 20 times larger than that of the van der Waals potential. Thus the threshold temperature for the former is $\sim 1 /(20)^{2}$ times that of the latter, i.e. from the discussion of ground state collisions, of the order of $1 \mu \mathrm{K}$. One can also appreciate that because the $C_{3} / r^{3}$ interaction is significant over a much larger distance, the height of the maximum in the long-range potential (that induced by the rotational energy) is much reduced, and thus more partial waves are able to contribute to the cross-section. The early studies of cold collisions predated the discovery of sub-Doppler temperatures in the MOT, and thus for the above reasons it was originally hoped that $n=3$ collisions might be modelled semiclassically with contributions from many values of $l$ smearing out quantum effects.

Gallagher and Pritchard [30] proposed such a model which has subsequently proved to be useful in understanding the frequency dependence of light-assisted collision rates. They envisaged that the loss rate comprises two factors. The first, and the most interesting, is a dynamic factor accounting for the rate at which atoms reach the small separation region in which the energy transfer (either fine structure change or radiative redistribution) is sufficient to cause trap loss. They separated this term into an excitation rate to the excited state curve, which is resonant at a particular separation $r$ depending on the laser field detuning, and an exponential survival probability against spontaneous emission whilst accelerating on the attractive potential. The second factor in the loss rate accounts for the energy transfer rate once the small separation region is reached, and was estimated by considering the molecule to continually traverse the at- 
tractive well until the occurrence of reradiation or a fine structure-changing transition. The dynamic factor gives the scaling of the loss rate with the laser field parameters. By replacing the multiple excited state curves with a single effective potential and assuming low saturation of the atomic transition, they predicted that the detuning dependence of $\beta$ should be [41]:

$$
\begin{gathered}
\beta \propto\left(\frac{\Delta}{\delta}\right)^{2} e^{-(\Delta / \delta)^{5 / 6}}(\delta \gg \Gamma) \\
\text { where } \Delta=\frac{C_{3}}{h}\left(\frac{\mu \Gamma^{2}}{3.59 C_{3}}\right)^{3 / 5} \approx 115 \mathrm{MHz} \text { for caesium }
\end{gathered}
$$

and that $\beta$ should increase linearly with the excitation rate to the excited state potential. Loss from fine structure-changing collisions was predicted to dominate over that from radiative redistribution. The form of Eq. (2.50) is readily understood. At first $\beta$ increases rapidly with increasing detuning because the survival probability is greater if the atoms are excited at a closer separation; for larger detunings (greater than approximately $\Delta / 2$ ) the loss rate decreases because of the reduction in numbers of pairs of atoms at small separations.

Sesko et al. [74] were the first to confirm unambiguously the existence of lightassisted collisions. The signature they looked for was the intensity dependence of $\beta$. As the trapping intensity was reduced (lowering the total saturation parameter from about 0.8 ) a linear decrease in loss rate was observed, until at very low intensities hyperfine-changing collisions set-in and the loss rate increased. The linear decrease was interpreted as the linear dependence of $\beta$ on the excitation rate. In fact, because the trap depth also varies with trapping intensity and because reradiative redistribution releases a spectrum of kinetic energies, a linear variation is only expected if fine structure-changing collisions are dominant. The same linear variation has also been observed by Wallace et al. [87] in rubidium (see also chapter 5). Since it is not possible to vary the frequency of the MOT trapping lasers by more than a few linewidths, Sesko et al. [74] investigated the frequency dependence of $\beta$ by the use of an additional "catalysis" laser. When the detuning of this laser was restricted to values greater than $\sim 5$ linewidths the effect of this additional light field was to produce an increased collisional loss rate but without disruption of the trapping process. In this way the "trap loss frequency spectrum" was probed. For caesium it was observed that the frequency variation of $\beta$ roughly follows Eq. (2.50), but that the detuning of the peak loss rate was a factor $\sim 6$ larger than expected, and the absolute rates a factor between 2 and 5 larger than predicted.

The Gallagher-Pritchard model was refined first to account for fine structure [49] and then saturation effects [5]. The modifications resulted in good quantitative agreement with the caesium data of Sesko et al. [74], but there was subsequently disagreement with similar measurements made on rubidium by Wallace et al. [87] and by Hoffman et al. [42]. Dulieu et al. [26] have recently shown that the agreement with caesium was accidental. The study of Hoffman et al. [42] also illustrated the profound effect of excited state hyperfine structure on trap loss spectra. For detunings less than the spread of the hyperfine structure $(\lesssim 450 \mathrm{MHz})$ the loss spectra for ${ }^{85} \mathrm{Rb}$ and ${ }^{87} \mathrm{Rb}$ were 
measured to be radically different, but at larger detunings no difference was observed. In the light of the Gallagher-Pritchard model this observation is not too surprising. The modification of the potential curves by the hyperfine interaction will change the spatial variation of the excitation rate, and additionally there is the possibility that more than one curve can be excited. Thus the collision dynamics can be expected to be considerably different.

Semiclassical treatments of light-assisted collisions are now known to give poor representation of the collision dynamics at sub-Doppler temperatures $[6,81]$. For example, interference effects induce a strong sensitivity to exact form of the potential curves [26]. Holland, Suominen and Burnett [44, 43] have studied the evolution of the time-dependent Schrödinger equation for a system of two-laser coupled potential surfaces, with spontaneous decay included by the stochastic Monte-Carlo method of Dalibard, Castin and Mølmer [21, 64]. This avoids direct numerical evaluation of the density matrix in two-dimensional position space which is not possible with current computing resources [53]. The method lends itself particularly well to the study of collisional heating from radiative redistribution, and has predicted significant heating at temperatures below the Doppler limit $T_{D}$, sensitive to both laser detuning and intensity. 


\section{Chapter 3}

\section{Experimental Setup}

\subsection{Introduction}

This chapter describes the features of the apparatus and measuring techniques that were common to most of the experiments. In this work we were particularly concerned with obtaining accurate measurements of the number of trapped atoms, and their spatial distribution and temperature under different trapping conditions. The measuring techniques developed to achieve this are fully described, including details of the data acquisition and computer control of the experiments. An overview is given of the laser system and optics and other apparatus used to operate the MOT. Much of the basic apparatus was similar to that described in [76]. A number of the modifications, mainly to the laser diodes, have already been documented in [16] and this material is not repeated here. In general detailed description of specific experimental aspects have been left to the appropriate sections of the later chapters.

\subsection{The diode lasers}

\subsubsection{General}

At $852 \mathrm{~nm}$ the wavelength of the caesium $D_{2}$ line coincides with the output of high power diode lasers. These convenient lasers have now become almost standard in laser cooling work with caesium and rubidium, and their use is becoming widespread in other atomic physics applications.

In our experiments there were two independent diode laser systems. The main trapping light was provided by a master-slave system which consisted of a gratingstabilised master laser that injection-locked a slave laser of high output power. In the alkalis the cooling cycle is never completely closed. Off-resonant excitation causes leakage of atoms to a lower hyperfine level (see chapter 6), and this was counteracted by a single laser which "repumped" the atoms back into cooling cycle. All the lasers were Spectra Diode Labs GaAs index guided devices rated at either $100 \mathrm{~mW}$ or $150 \mathrm{~mW}$, although for the dark MOT experiments a $50 \mathrm{~mW}$ DBR diode laser (also SDL) was used as the repumping source. 
Both master and repumping lasers employed an extended cavity to reduce the laser linewidth and sensitivity to optical feedback, and to allow for frequency selection. Optical feedback was from the first order of a reflection diffraction grating which had 1200 lines per $\mathrm{mm}$, was gold coated and blazed for first order at $750 \mathrm{~nm}$, and was positioned at a distance of about $3 \mathrm{~cm}$ from the laser chip in the Littrow configuration. The grating was aligned parallel to the electric field vector to give a maximum output transmission into zeroth order $(\sim 40 \%)$, and with this alignment feedback was $\sim 35 \%$. Screw adjustments enabled coarse alignment of the grating whilst piezos provided fine alignment. The procedure for collimation of the highly divergent output beam and subsequent alignment of the grating for reliable operation is described in both [76] and [16]. With the setup described it was possible to scan the laser wavelength manually with the grating over some $10 \mathrm{~nm}$, with single mode operation typically observed over $1 \mathrm{GHz}$. The laser linewidth in this arrangement should be less than $1 \mathrm{MHz}$, but is sensitive to acoustic noise.

A severe disadvantage of the cavity-extended diode laser is the slow drift in its frequency caused by thermal change of the extended cavity length. The laser and grating were mounted on an invar base which was thermo-electrically cooled, in addition to the temperature stabilization applied to the laser chip itself. Even with these measures the laser frequency without active stabilization was observed to drift at a rate typically faster than $1 \mathrm{GHz}$ in 10 minutes (although noticeably slower when the lab was empty). The stabilization method employed to overcome this drift is described below. Two main designs of grating mounting were used during the course of the experiments, with differing consequences for the frequency drift. One was a three-point mirror mount with low voltage piezo stacks $(75 \mathrm{~V})$ and Allen key adjustments at three corners (Photon Control ASM-15, ASM-20). The provision of vertical piezo control allowed easy optimization of the lasing threshold current on a day-to-day basis, but the mount itself was sensitive to fluctuation in external conditions in both horizontal (frequency tuning) and vertical directions. The piezo material had a much larger thermal expansion than the invar base (even considering the relative lengths) but there also appeared to be drifts from electrical heating of the piezo and from the Photon Control voltage source itself. A second arrangement utilised a high voltage piezo stack (500 V) attached to a secure two-point mirror mount. The grating was mounted on the end of the piezo to give movement along the cavity direction. It was necessary to align the grating manually and this would have been considerably easier with differential adjustment. However, once the threshold current had been minimized this mounting arrangement was generally observed to be the more stable of the two. All lasers were housed inside containers to shield from thermal air currents. This improved stability considerably but the performance was fairly independent of the type of container - a simple cover made from plastic and wood was as good as airtight.

\subsubsection{Spectral characteristics of the slave laser}

For understanding the behaviour of the MOT it was important to have a knowledge of the spectral profile of the trapping light. Nominally the cavity-extended master 
laser has a linewidth considerably less than that of the $5.22 \mathrm{MHz}$ natural linewidth of the $6 \mathrm{P}_{3 / 2}$ level in caesium, but it does not follow that this should always be true for the slave, particularly if the injection-locking is poor. The theoretical locking range depends on the slave intensity and the intensity injected, and a typical prediction for a diode laser is of order $1 \mathrm{GHz}$ [16], which is equivalent to a couple of milliamps. This is indeed what was observed although the locking had a tendency to become very sensitive when the injecting beam was not sufficiently well-aligned or was of low intensity.

The spectral distribution of the slave laser was investigated with a $1 \mathrm{GHz}$ spectrum analyser of finesse $\sim 200$. The appearance of the Doppler absorption profile, which was the means usually used to assess the quality of injection-locking, was noted simultaneously. The open circles in Fig. 3.1 show the spectral distribution with sidebands minimized by optimizing the slave current. The corresponding Doppler absorption signal was perfectly clean. The solid line is a best fit Lorenzian with a FWHM of about $4 \mathrm{MHz}$, which is very similar to the nominal instrumental width of the analyser and is of the order of the expected linewidth of the laser. Thus the profile gives an upper limit to the slave linewidth. The small sidebands that appear in Fig. 3.1 at $\pm 80 \mathrm{MHz}$ were induced by stray radiofrequency fields from (shielded) acousto-optic modulator drivers. Importantly, the only effect on the spectral distribution when the slave current was changed slightly so as to induce obvious noise on the Doppler absorption profile, was that the size of these sidebands were much increased. That is, the width of the Lorenzian was left unaltered.

\subsubsection{Frequency stabilization}

Frequency reference signals for master and repumping lasers were generated from Doppler-free saturated absorption, obtained with two similar optical arrangements [16]. The laser frequency was dithered (see below) to enable a phase-sensitive detector to generate a dispersion-shaped error signal, centred on the appropriate hyperfine transition. This error signal was fed back onto the grating piezo via an integrator with a time constant of about $1 \mathrm{~s}$.

The exact method for dithering and integrating the frequency error signal differed for the two lasers. For the repumping laser the piezo voltage was a $1 \mathrm{kHz}$ oscillator which produced a peak-to-peak dither amplitude of $10 \mathrm{MHz}$. In early experiments light from the master laser was passed through a low frequency AOM before directed into the saturated absorption spectroscopy, and dither was imposed by oscillating the AOM driving frequency. The error signal was integrated by computer. The were two major disadvantages with this setup. First, the saturated absorption spectroscopy optics needed to be realigned whenever the AOM frequency was significantly changed. Thus the only means to change the trapping frequency transiently was to alter the voltage on the master piezo, but in doing this the locking point was lost. The unlocked frequency was observed to drift over several $\mathrm{MHz}$ during a period of a few seconds, which was very unsatisfactory for precise measurements of time-varying MOT properties such as number and volume. Secondly, the laser had to be left unlocked when the computer, which was also used to control experimental sequencing, was asked to perform a differ- 


\section{Normalized spectrum analyser signal}

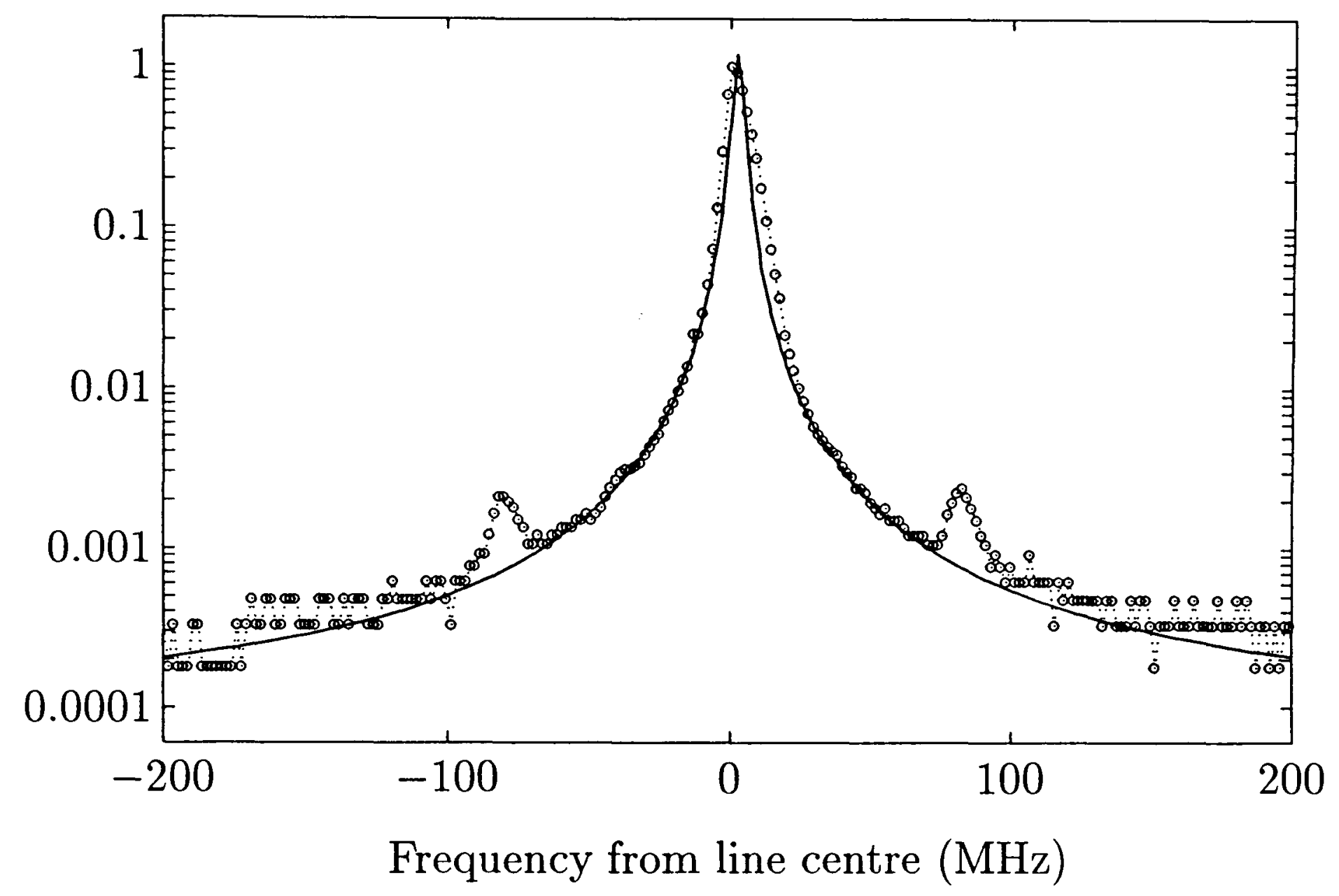

Figure 3.1: Spectral profile of an injection-locked slave laser as seen with an optical spectrum analyser of instrumental width $\sim 5 \mathrm{MHz}$ (open circles). The slave current was that which optimized the Doppler absorption profile. The solid line is a best fit Lorenzian, and has a FWHM of $4 \mathrm{MHz}$. An offset of $10^{-4}$ was added to the analyser signal such that plotting on a logarithmic scale was possible; the Lorenzian has been equally offset.

ent task. For example, during early temperature studies the laser was left unlocked for about $50 \mathrm{~ms}$ during the time-of-flight routine whilst the detuning and intensity of the trapping beams were altered, and this was long enough to cause significant scatter in the data. This rather idiosyncratic design was replaced by a system with a standard integrating circuit and with an AOM between the master and slave lasers, thereby making it possible to keep the master laser locked to the line at all times. The frequency dither was produced by oscillating the laser current $( \pm 1 \mathrm{MHz}$ at around $10 \mathrm{kHz})$, and the trapping frequency could then be switched by changing the AOM driving frequency.

With the above locking schemes it was generally possible to maintain the laser frequencies to $\sim \pm 1 \mathrm{MHz}$ for periods of hours between mode hops. 


\subsection{The optical setup}

\subsubsection{General features}

The lasers and associated optics for the saturation spectroscopy, frequency shifting and shuttering of the beams were arranged on an optical table. The light was transported to the optics for the trap itself through single-mode optical fibres which gave excellent quality intensity profiles and removed the necessity of realigning the trapping optics on a daily basis; both of these features were important for attaining consistent density measurements (see Sec. 4.8). Frequency detunings relevant to the caesium $6 \mathrm{P}_{3 / 2}$ hyperfine structure were obtained by single or double passing light through one or two acoustic-optic modulators (up to $125 \mathrm{MHz}$ ), and these also enabled precise control of intensity and also rapid switching.

The important requirements for the light in the measurements presented in this thesis were (a) trapping light whose detuning could be rapidly increased from a value $\delta \approx 2 \Gamma$ (for loading), up to $\delta \sim 12 \Gamma$, and whose intensity could be rapidly reduced down to some small fraction (say 0.2 ) of a saturation intensity per beam (i.e. a total trapping intensity of $\sim 3 \mathrm{mWcm}^{-2}$ ). In both instances the final laser parameters needed to be fixed accurately for several seconds or longer. (b) The option to repump on either $F=3 \rightarrow 3^{\prime}$ or $F=3 \rightarrow 4^{\prime}$, again with the facility to reduce the intensity, (c) a timeof-flight probe beam at resonance with $F=4 \rightarrow 5^{\prime},(\mathrm{d})$ the provision of additional repumping light (also of switchable intensity) and of light at resonance with $F=4 \rightarrow 4^{\prime}$ ("depumping" light) for the dark MOT.

The optical layout designed to meet the above requirements is shown in Fig. 3.2. The arrangement of trapping optics around the vacuum chamber is shown in Fig. 3.3, with the detailed layout of the dark MOT optics (chapter 6) included for completeness. It should be pointed out that during the course of the eighteen months of experiments the arrangements on both tables were almost continually being altered as the need arose.

\subsubsection{Details of the optical and laser systems}

The light from each laser diode was collimated with an anti-reflection coated plastic aspheric lens, designed specifically for laser diodes (Corning P.M.O. 350110), and a pair of anamorphic prisms (Melles Griot) was used to make the elliptical beam crosssection more circular. Each laser was optically-isolated with a home-designed isolator (for design details see [76]), with isolation deduced to be only about $-10 \mathrm{~dB}$. The DBR laser was far more sensitive to optical feedback and it was used in conjunction with a commercial isolator providing $-30 \mathrm{~dB}$ of isolation (Halbo Optics 1579-7).

After the optical isolator a small fraction of the beam from the master laser was split off for saturation absorption spectroscopy to permit the laser to be locked on resonance to the $F=4 \rightarrow 5^{\prime}$ transition at all times. The remainder of the light passed twice through an AOM (Isomet) to shift its frequency down by $170 \mathrm{MHz}$ before injection-locking the slave. The light from the slave was shifted up in frequency by $160 \mathrm{MHz}$, again by a double pass AOM (lsomet), to give a net shift of $-10 \mathrm{MHz}$, 


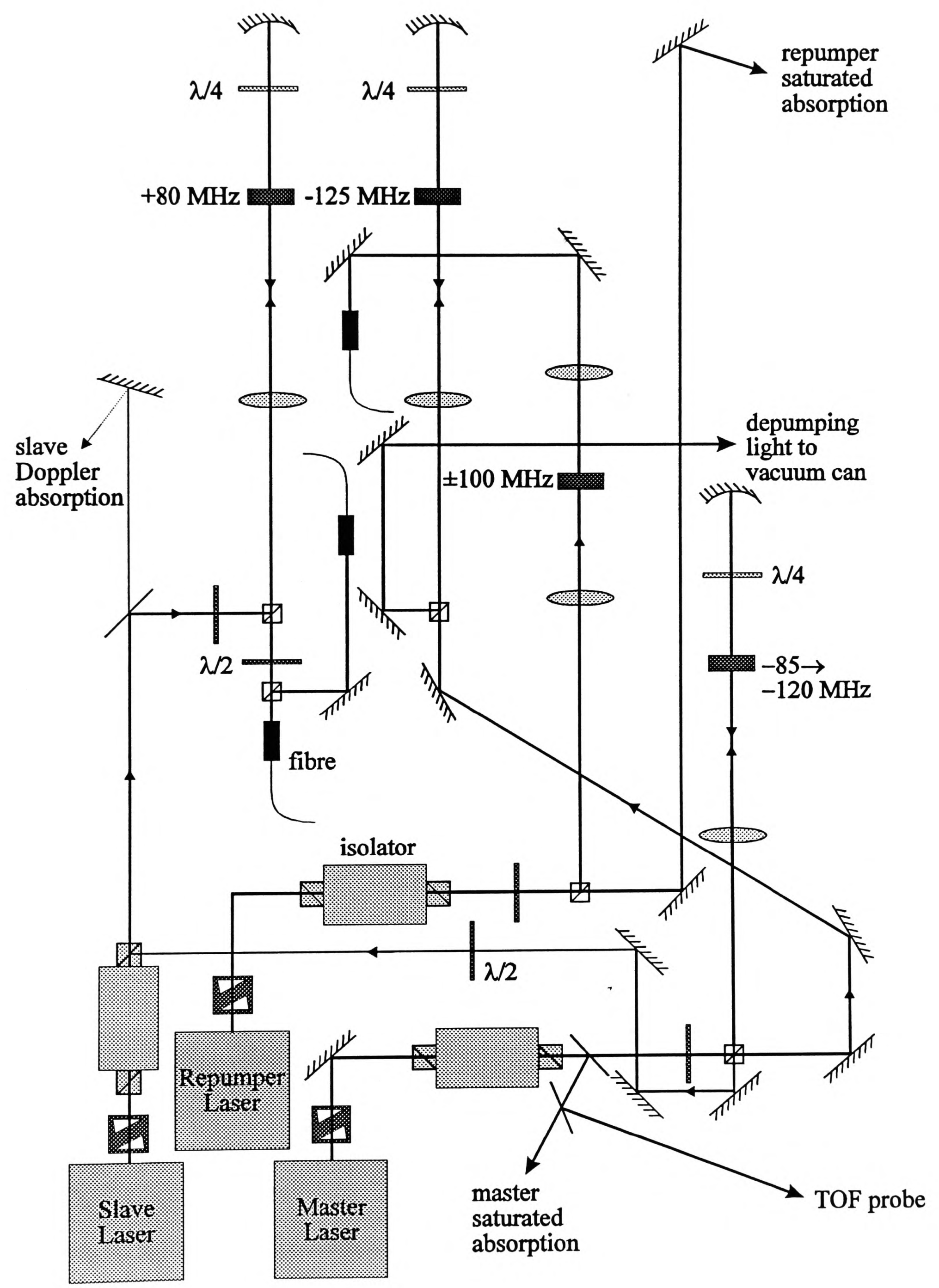

Figure 3.2: Arrangement of optics on the laser table. 


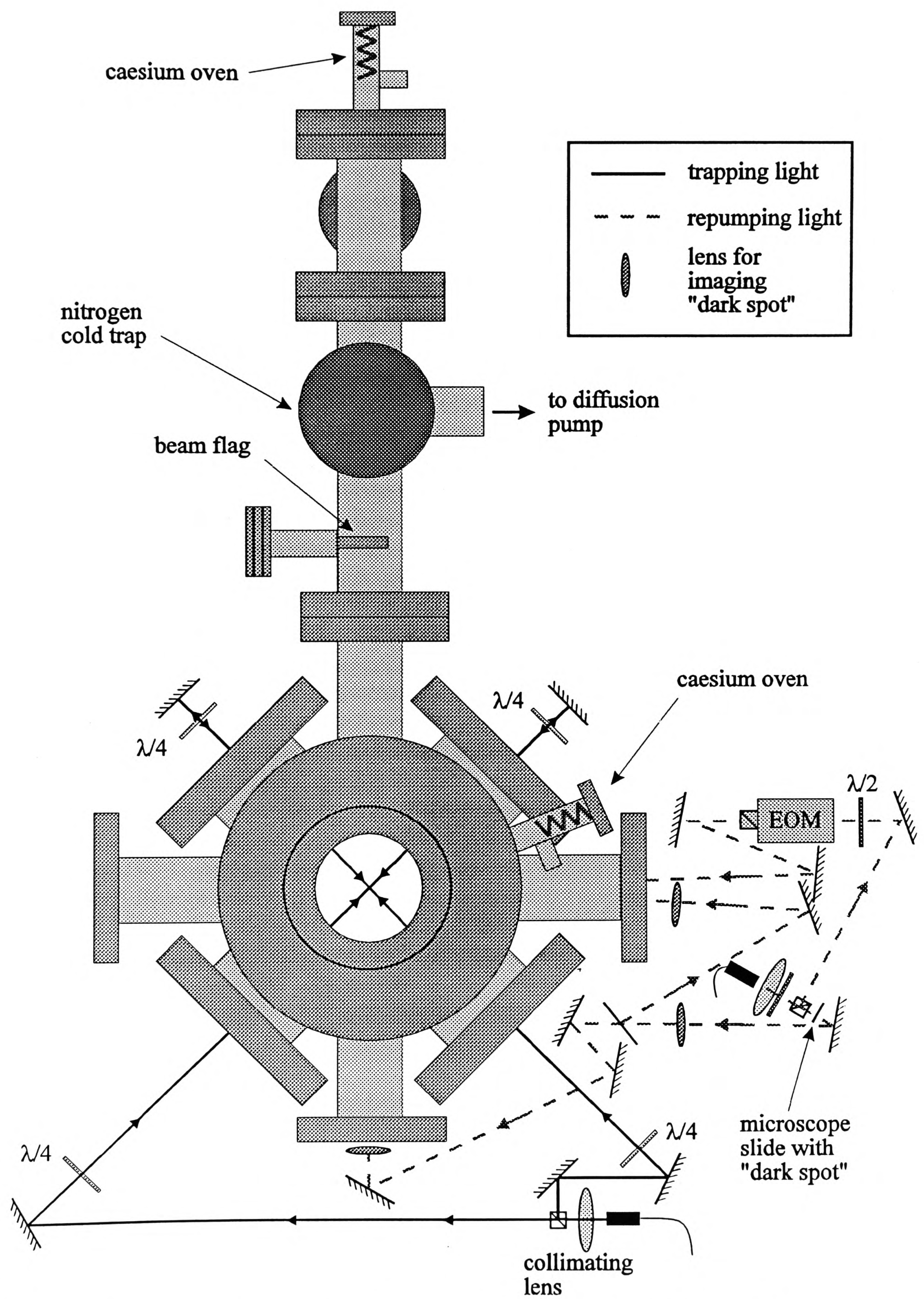

Figure 3.3: Plan view of the vacuum system and trapping optics. The arrangement of optics for the repumping light was that used for the spatial dark MOT. 
the hence the detuning for loading the MOT. Increasing the driving frequency of the master AOM in this arrangement gave a wide range of trap frequencies. Furthermore, double passing the AOM meant that the alignment of the master light into the slave laser remained almost unaltered with change of frequency, so that it was possible to transiently switch the detuning and simultaneously retain good injection-locking. The AOM through which light from the master passed had a centre frequency at $110 \mathrm{MHz}$ at which frequency the trap detuning was $-60 \mathrm{MHz}(\delta \approx 11.5 \Gamma)$, and trap detunings in excess of $-80 \mathrm{MHz}$ were readily accessible.

After the light from the slave had been passed through the AOMs it was split into two beams which were transported separately by optical fibres to the vacuum chamber, and the output from these formed the horizontal and vertical trapping beams. All fibres used in the experiments were single-mode and nonpolarization-preserving, supplied by Point Source. They had mode-matching lenses permanently attached to the input end, and typical transmissions were $50 \%$ for $1.5 \mathrm{~mm}$ diameter input beams. At the vacuum chamber light from the two fibres was collimated ${ }^{1}$ to produce beams of approximately $10 \mathrm{~mm} 1 / e^{2}$ diameter (accurate measurements the of beam sizes are recorded in [16]), and the horizontal beam split to generate trapping light along orthogonal horizontal directions. All three independent trapping beams were aligned to intersect at the magnetic field zero, with retroreflections superimposed upon the initial directions to an angular accuracy of better than $5 \times 10^{-4} \mathrm{rad}$ (judged by eye); the alignment procedure is described in Sec. 4.8.

The facility to repump on either $F=3 \rightarrow 4^{\prime}$ or $F=3 \rightarrow 3^{\prime}$ was made possible by locking the repumping laser to the highest frequency crossover peak in its saturated absorption spectrum, and then single passing its light through an AOM driven at $100 \mathrm{MHz}$ (Crystal Techology). Either the +1 or -1 order was optimized depending on the required frequency. The repumping light was transported to the vacuum chamber by a third optical fibre.

The frequency of the time-of-flight probe needed to be close to the $F=4 \rightarrow 5^{\prime}$ resonance. This light was conveniently obtained directly from the master laser, before its frequency was shifted. In the work on the dark MOT $F=4 \rightarrow 4^{\prime}$ light was required. This was obtained by passing a beam from the master laser twice through an AOM (Crystal Technology) to shift its frequency down by $251.4 \mathrm{MHz}$ from the $F=4 \rightarrow 5^{\prime}$ resonance. This light was expanded to a diameter of $3 \mathrm{~mm}$ and directed straight into the vacuum chamber.

\subsubsection{Frequency and intensity control}

The logic for the frequency and intensity control of the trapping beams is shown in Fig. 3.4. To generate the $-10 \mathrm{MHz}$ detuning used to load the MOT the master AOM was driven at $85 \mathrm{MHz}$ by an Isomet D302B voltage-controlled oscillator, and the slave AOM at $80 \mathrm{MHz}$ by a Gooch and Housego MDA080-1 fixed frequency oscillator. Both rf signals were amplified by $29 \mathrm{~dB}$ to generate $\sim 2 \mathrm{~W}$ of driving power. Selecting a

\footnotetext{
${ }^{1}$ Strictly the horizontal beams were made to converge slightly to account for a typical $15 \%$ intensity imbalance between a trapping beam and its retroreflection.
} 


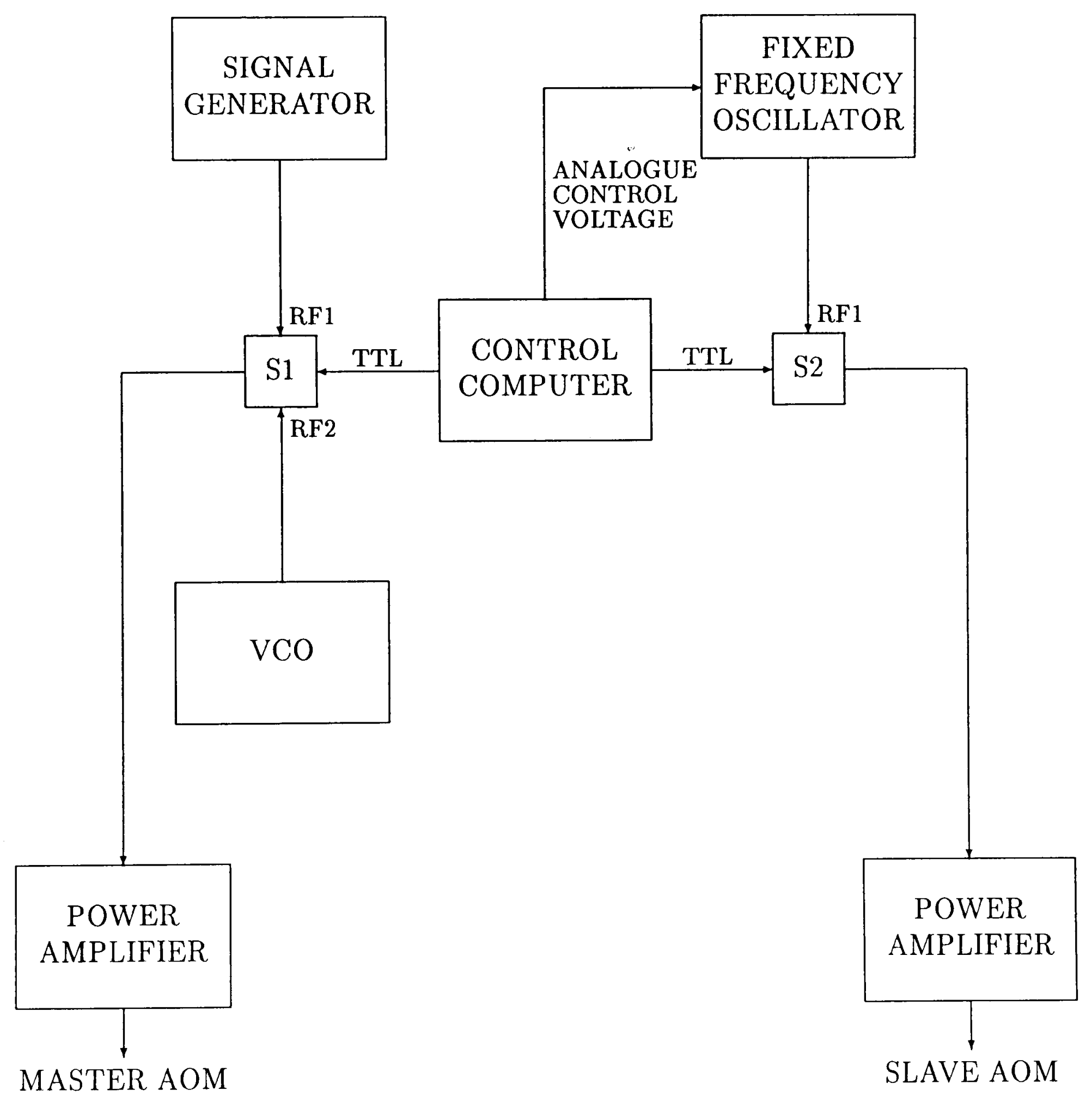

Figure 3.4: Arrangement for frequency and intensity control of the trapping beams. Switches S1 and S2 were respectively used to select one of two detunings and to extinguish the light. 
second detuning was achieved by switching the master AOM driving signal to that from a Marconi 2019A signal generator, whose frequency was preselected remotely by GPIB link with the control computer. The switch was a Minicircuits ZSDR-230, activated by a TTL signal from the computer. This method enabled sub-millisecond switching (much faster that the Marconi itself could change frequency).

The trapping intensity was varied by changing the activation voltage to the slave AOM driver, and thus the power supplied to the AOM. To generate a voltage-tointensity calibration the computer sampled the signal from a photodiode placed in one of the trapping beams whilst smoothly varying the driver voltage. The resulting lookup table enabled rapid switching to a chosen intensity level. This was rather essential because the trapping intensity was observed to vary with activation voltage in a fashion which was highly non-linear and which tended to change on a daily basis. For time-of-flight measurements it was important to be able to extinguish completely the trapping light. This was the purpose of the second Minicircuits switch shown in Fig. 3.4 which provided an extra $-10 \mathrm{~dB}$ of isolation.

A problem with altering the driving power to an AOM is that for a period of seconds after a switch the angular deflections of the AOM orders tend to wander slightly due to thermal effects. In the optical setup shown above this caused a change of coupling into the optical fibres and consequently a variation in the trapping intensity, sometimes by as much as a factor of two. For both the density and cold collision measurements it was important to be able to switch to an accurately constant intensity. The problem was solved almost completely with a simple positive feedback scheme. After switching, a tiny fraction of the trapping light was measured with a photodiode in both the horizontal and vertical directions and the signals sampled by the computer. An average error signal was calculated and a correction voltage sent to the AOM supply. Inevitably it was impossible to correct exactly for the differing drifts induced in two AOMs by changing their driving powers by the same amount. However, typically the drift of the total intensity was minimized to less than $5 \%$ during a period of $10 \mathrm{~s}$.

The AOM generating the repumping frequency was driven at $100 \mathrm{MHz}$ by a second voltage-controlled oscillator, and that for the depumping light at $125 \mathrm{MHz}$ by a Marconi TF 2015 signal generator. Power amplification was as described above. It was necessary to be able to vary the intensities of both these frequencies. Clearly this had to be done in a different way to the method described above for the fixed frequency oscillator. The approach in both instances was to attenuate the rf signal before amplification with a Minicircuits ZMAS-3 current-controlled attenuator. A simple op-amp circuit converted an analogue voltage from the computer into the required current. Stabilization of both repumping and depumping intensities was with procedure very similar to that for the trapping intensity.

\subsubsection{Trapping beam quality}

An important consideration during the study of the MOT density was the sensitivity of the compression to the quality of the trapping beam wavefronts and polarizations. The former was assessed by profiling the trapping beams with a CCD after the insertion 
of various relevant optical elements. To minimize uncertainty in assigning the origin of imperfections neutral density filters were inserted before the optical fibres and the protective glass plate in front of the CCD was removed (this led to interference patterns with the laser light).

Observations were the following. When sampled immediately after collimation from the fibre the trapping beam profiles were good approximations to Gaussian shapes, with deviations typically less than $5 \%$. However, at distances of greater than $1 \mathrm{~m}$ (distance from fibre to MOT for each retroreflected trapping beam was around $3.5 \mathrm{~m}$ ) the quality of the collimating lens became significant. Initial experiments used a plastic aspheric (Comar 80AP64) but this was observed to introduce a bright edge to the beam, probably due to spherical aberration. The best replacement was found to be a glass projection lens (diameter $7.6 \mathrm{~cm}$, focal length $6.5 \mathrm{~cm}$ ) which, for accurate collimation, gave no sign of distortion.

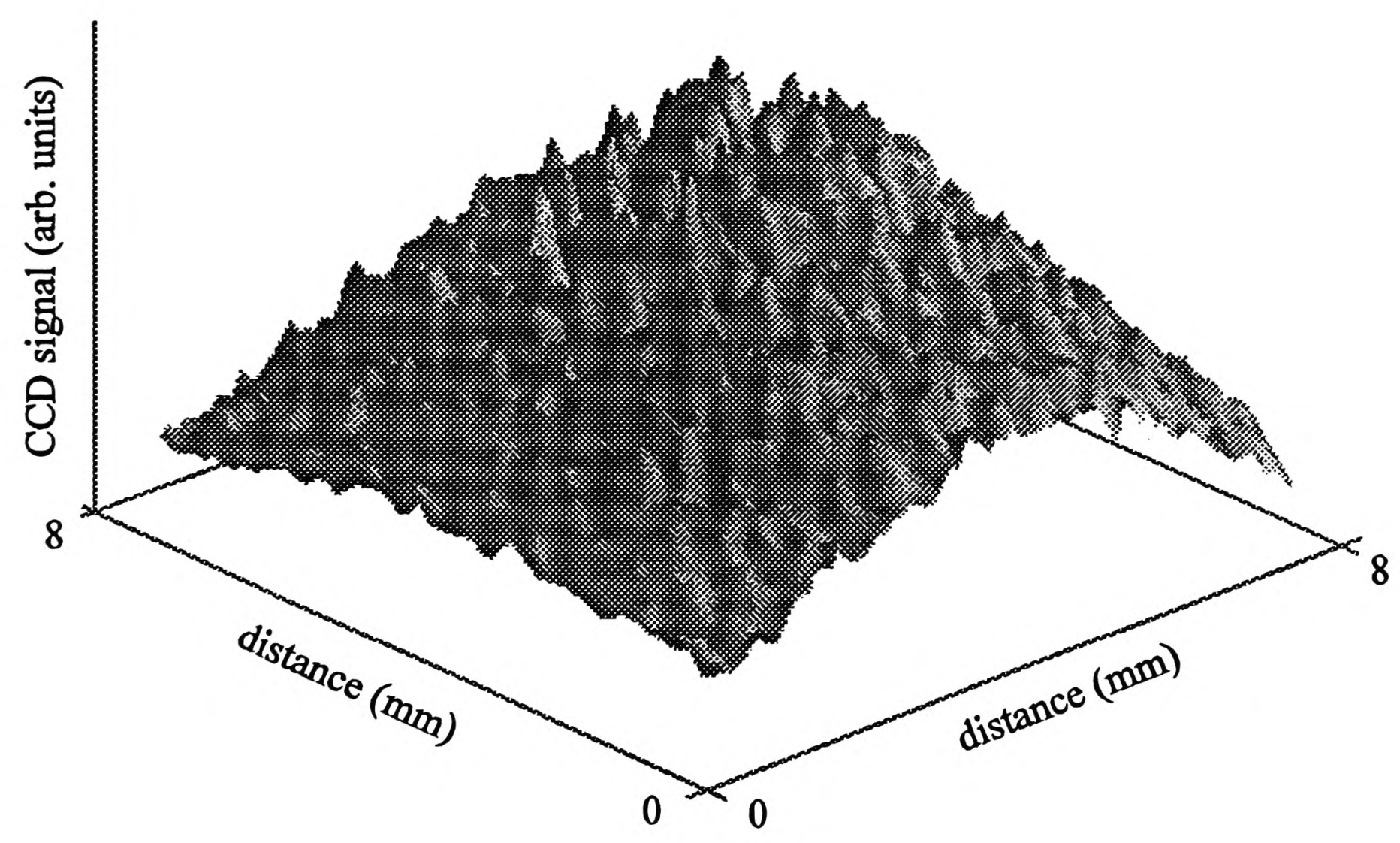

Figure 3.5: Trapping beam profile after passing through collimating and polarizing optics and once through the vacuum chamber.

Both mirrors (flat to $\sim \lambda / 10$ ) and polarizing beam-splitting cubes (TecOptics) caused noticeable imperfections to the spatial profiles. At least some of this was due to aperturing of the beams from the 1" optics: diffraction rings were clearly visible and their separations were typically $100-200 \mu \mathrm{m}$, compatible with the difference in Fresnel zone radii for the apertures and distances considered. These patterns were minimized but not entirely eliminated by careful positioning of the optics. Diffraction patterns due to visible imperfections on the optical surfaces were also noticed. The beam-splitting 
cubes were observed to introduce additional interference patterns, presumably due to reflections from front and back surfaces, and the similar was true for mirrors without ground glass backs.

Fig. 3.5 shows a beam profile after passing through collimating lens (glass), zero order half waveplate (Fichou), beam-splitting cube, multiple order quarter waveplate and two vacuum chamber windows (anti-reflection coated and flat to $\sim \lambda / 5$ ) and at a distance of about $2.75 \mathrm{~m}$ from the fibre. It was not desirable to introduce unnecessary distortions by focussing the trapping profile onto the CCD array and thus only the central region of the profile is shown. The figure shows that fluctuations are typically $5-15 \%$ on spatial scales that range from $100-400 \mu \mathrm{m}$. Effects of these fluctuations are referred to in chapter 4.

The effect of the MOT on the profile of a retroreflected beam was investigated. A retroreflecting mirror was replaced by a beam splitter that transmitted a small fraction of the incident trapping beam, and this was imaged. The diffraction pattern introduced by the scattering of $10^{7}$ atoms at a low detuning $(\delta=2 \Gamma)$ is shown in Fig. 3.6. The decrease of intensity at the beam centre is roughly $30 \%$, but at large detunings this should fall-off as the inverse of the absorption cross-section, i.e as $\delta^{2}$.

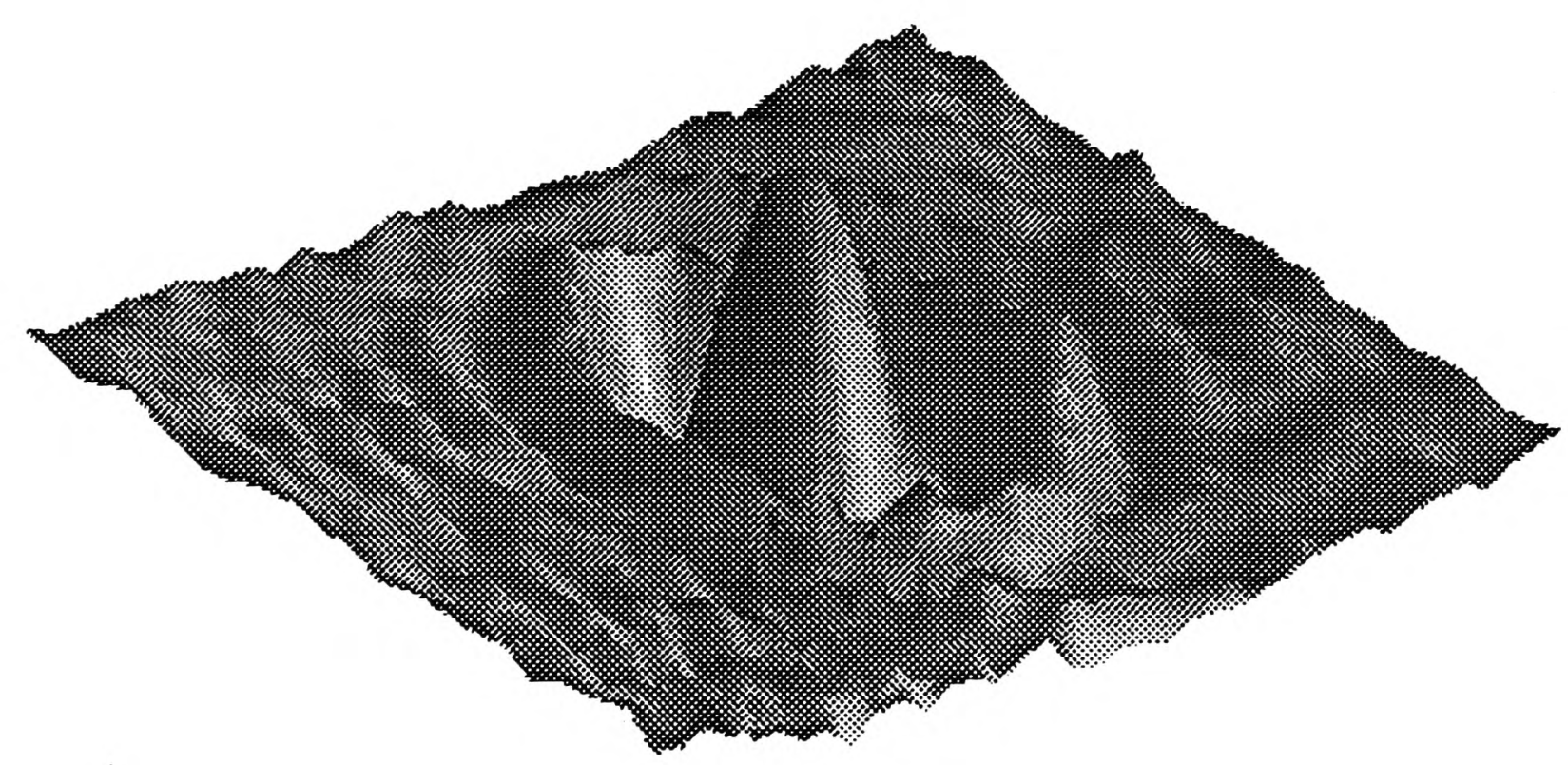

Figure 3.6: The diffraction pattern in a retroreflected trapping beam caused by absorption of light by the MOT. The image shown was that obtained by subtracting the intensity profile of the beam with the trap present from that with no trap present. The peak at the centre is thus an intensity minimum, and in the figure (for $10^{7}$ atoms at $\delta=2 \Gamma$ ) is roughly $30 \%$ of the peak intensity. The dimensions of the profile are $8 \times 8 \mathrm{~mm}$. 


\subsection{Other trapping apparatus}

\subsubsection{The vacuum chamber}

The MOT was formed at the centre of a large stainless can built by VG Special Systems, pumped by an oil vapour diffusion pump (Edwards E04K), and with the provision of a liquid nitrogen-cooled shield [76]. The MOT lifetime under conditions of vapour loading was typically in the range $0.25-1.5 \mathrm{~s}$ which suggests that accessible pressures were in the range $10^{-9}-10^{-8}$ Torr. For time-of-flight measurements (see below) a sixway cross was added to the bottom of the chamber the centre of which was at a distance of $42 \mathrm{~cm}$ from the magnetic field zero.

\subsubsection{The caesium source}

Two methods of trap loading permitted a wide range of steady-state trap numbers to be attained. The first was to capture atoms released from a caesium oven attached to the side of the chamber [76]. When the oven was run substantially above room temperature for a period of a few days the sides of the chamber became coated with caesium, and the trap loaded from a background vapour with a pressure of probably a few $10^{-8}$ Torr. This typically resulted in the loading of between $10^{7}$ and $10^{8}$ atoms, with trap lifetimes in the range quoted above. The intensity imbalance due to absorption by the background caesium vapour was typically $15 \%$ and, as mentioned previously, was compensated in the horizontal direction by slightly converging the beams. On the other hand, if the sides of the chamber were not sufficiently coated then the trap would load directly from the beam of atoms effusing from the oven. This typically gave less than $10^{7}$ atoms with an average pressure of probably less than $10^{-8}$ Torr, as on occasion lifetimes of up to $5 \mathrm{~s}$ were observed. The second method of loading was to trap directly from an unslowed thermal atomic beam, effusing from an oven positioned about $1 \mathrm{~m}$ from the trap centre. Varying the flux of atoms in the beam, changing the oven temperature or partial shuttering of the beam, enabled the trap number to be varied in the range $10^{4}-10^{7}$, and trapping beam imbalances were reduced to only a few percent. However, lifetimes were never observed to exceed $5 \mathrm{~s}$.

\subsubsection{The magnetic field coils}

Early experiments used a coil design that produced a magnetic field gradient of just over $2 \mathrm{Gcm}^{-1} \mathrm{~A}^{-1}$ [13], but continuous currents were limited to $4 \mathrm{~A}$; above this there was heating which led to outgassing and an observable decrease in the trap lifetime. For the majority of the experiments a better coil design was used whose specific details are given in [16]. These coils had 60 copper turns each and were separated by a distance roughly equal to the coil diameter of $2.6 \mathrm{~cm}$. To give good heat dissipation the coils were wound around massive copper formers and supported by thick copper bars, but significant heating was still observed for continuous operation above $4 \mathrm{~A}$. However since they produced $10 \mathrm{Gcm}^{-1} \mathrm{~A}^{-1}$, as measured with a Hall effect probe, the coils gave gradients up to $40 \mathrm{Gcm}^{-1}$. 


\subsubsection{Nulling coils}

The effect of an external magnetic in one dimension was described in chapter 1 . The friction force cools to a finite velocity given by:

$$
v_{B}=-\frac{g_{J} \mu_{B} B}{\hbar k}
$$

(note comparison with Eq. 2.8). The magnitude of the Earth's magnetic field is of order $0.5 \mathrm{G}$, and for caesium this implies from Eq. (3.1) a heating of order several hundred microkelvin. By comparison temperatures of below $3 \mu \mathrm{K}$ have been measured in caesium molasses [73] which implies that the Earth's field must be nulled to an accuracy of order $10 \mathrm{mG}$.

Three pairs of coils surrounding the vacuum chamber were used to cancel the field. Originally the zero was found by removing the chamber and observing the Hanle effect in a rubidium vapour cell positioned at the centre of the coils [76]. This was accurate to $\sim 1 \mathrm{mG}$ but the reintroduction of the chamber changed the field by an unknown amount, estimated to be of order $10 \mathrm{mG}$. A more satisfactory approach is to minimize the temperature of atoms by varying the current through the coils. The time-of-flight signal was observed at conditions of large detuning and low intensity such that a minimum MOT temperature in the range $3-10 \mu \mathrm{K}$ was expected (the exact temperature depending upon the optical thickness of the cloud - see Sec. 4.4), and the currents then adjusted until this was observed. Typically at $3 \mu \mathrm{K}$ the zero was found to better than $0.5 \mu \mathrm{K}$ which, from the sensitivity of temperature to magnetic field measured in [16], implies that the Earth's field was cancelled to $\sim 5 \mathrm{mG}$.

\subsection{Measuring techniques and data acquisition}

\subsubsection{Fluorescence detection}

The trap number was deduced by calibration of the scattered fluorescence for reasons outlined in chapter 4 .

In early experiments light was detected with a Radio Spares RS 651995 photodiode. The light was collected with an uncoated aspheric lens (Melles Griot) and then split so that an image was simultaneously formed on a camera and on the photodiode. The lens was positioned just beyond a vacuum chamber window to enable its diameter of $6.6 \mathrm{~cm}$ to maximize the collection solid angle $\left(\eta / 4 \pi \sim 3 \times 10^{-3}\right)$, and with a focal length of $15 \mathrm{~cm}$ imaging was made $1: 1$. The photodiode had an active area of $41.3 \mathrm{~mm}^{2}$ which represented a compromise between being able to maintain signal for a variation in trap position of up to few millimetres (see Sec. 4.7), and minimizing stray noise and junction capacitance. The current from the photodiode was converted into a voltage $V$ across a feedback resistor $R_{f}$ of an op-amp, and the number of atoms $N$ was then deduced by the formula

$$
N=\frac{V}{R_{f}(\eta / 4 \pi) R_{\text {scat }} \hbar \omega \alpha(1-f)}
$$


where $R_{\text {scatt }}$ is the photon scattering rate, $\alpha$ the radiant sensitivity of the photodiode (amps per watt), and $f$ the fraction of light lost through the collection optics and caesium vapour. Careful estimates were made of $f$ and $\eta$, and $R_{\text {scatt }}$ was deduced with the approach described in Sec. 4.3. From uncertainties in the knowledge of $R_{f}, \alpha, f$ and $\eta$ it was estimated that the scattered power was measured to an accuracy of about $15 \%$. With $R_{f}=20 \mathrm{M} \Omega$ the photodiode response time was $100-200 \mu \mathrm{s}$, and the limit to detection was about $10^{6}$ atoms (at a high scattering rate).

For a number of experiments it was necessary to measure trap fluorescence substantially weaker than the photodiode system described above was capable of detecting, and often to combine this simultaneously with knowledge of the spatial distribution of the trap. It was thus convenient to obtain both measurements from a CCD. Standard video fields integrate intensity over a $20 \mathrm{~ms}$ period and typically this allowed less than $10^{4}$ atoms to be detected (at a high scattering rate) with a collection angle more than 20 times smaller than that for the photodiode. Nearly all measurements of number in this thesis were made with CCD detection.

The camera used was a CCIR monochrome Cohu 4750 which is one of the most sensitive unintensified detection devices available. It was calibrated for scattered power in the following way. A pinhole was illuminated with a caesium discharge lamp and imaged onto the CCD with a telephoto lens (the intensity was reduced by neutral density filters). The image was captured with a frame grabber (Sec. 3.5.3) and the total pixel count induced by the light calculated. This was converted into a power calibration by measuring the power incident onto the CCD with a calibrated power meter. The resulting conversion was accurate to about $10 \%$. With the additional uncertainty in determining the collection solid angle the power deduced from the CCD was estimated to be similar in accuracy to that deduced from the photodiode. This was tested by deducing the trap number simultaneously with the two devices. Agreement was found to about $20 \%$, thus confirming the independent calibrations and their stated accuracy.

\subsubsection{Trap imaging}

For reasons discussed in chapter 4 the MOT was nearly always viewed simultaneously with two cameras. These pointed in orthogonal horizontal directions at $45^{\circ}$ to the trapping beams.

To measure the cloud size of $10^{4}$ atoms or less it was necessary to be able to resolve trap diameters of order $100 \mu \mathrm{m}$. There were potentially three factors affecting the quality of imaging. First, for large lens apertures spherical aberration is significant and causes off-axis rays to be spread in the image plane. Secondly, for apertures that are too small diffraction will limit resolution. Finally, for a given magnification the ultimate limit to resolution is determined by the size of the CCD pixels.

The suitability of different lenses was investigated. Initially it was hoped that a single aspheric lens would be of sufficient quality, and an aspheric was tried in the 1:1 imaging setup described above. It was found, however, that that unless the lens was apertured down to less than the central $1 \mathrm{~cm}$ the smearing appearance from spherical 
aberration of an image size of order $0.5 \mathrm{~mm}$ (such as was produced by $10^{8}$ atoms at a density of $10^{10} \mathrm{~cm}^{-3}$ ) was very noticeable. The effect of spherical aberration is to redistribute energy from the high to the low intensity regions and thus misrepresent the spatial distribution of atoms, and it will become apparent in chapter 4 that this was very undesirable for a number of the measurements. Moreover, to reduce the spherical aberration sufficiently to adequately image $10^{6}$ atoms it was necessary to aperture the lens to just a few millimetres, resulting in a considerable reduction in image intensity and a regime where diffraction effects were beginning to become important.

Only by using compound lenses specifically designed to minimize aberration effects was the quality of imaging found to be sufficient for the smallest traps. For the majority of measurements the $x$ direction was imaged by a high quality $70 \mathrm{~mm}$ telephoto lens (Xenar) ground and coated for the visible, and the $y$ direction by a telescope arrangement of two identical achromatic doublets of focal length $25 \mathrm{~cm}$ (Melles Griot 01 LA 229) with a companion aplanatic meniscus lens (Melles Griot 01 LAM 229) to shorten the focal length to $14.76 \mathrm{~cm}$. The resolution limit of the two lenses was tested by viewing the image of a raster of $30 \mu \mathrm{m}$ spacings, illuminated by a caesium discharge lamp and positioned at the lens-trap distance $(\approx 28 \mathrm{~cm})$. Performance was quantified by defining the contrast of the imaged raster to be $\left(I_{\max }-I_{\min }\right) /\left(I_{\max }+I_{\min }\right)$. Fig. 3.7 shows the variation in contrast of the Xenar image with $f$-stop. One sees that the resolution was most seriously degraded at small apertures, and a simple order of magnitude estimate of the Rayleigh criterion indeed suggests that spreading on this spatial scale is to be expected for apertures of only a few millimetres. Interestingly at large aperture, where the effect of spherical aberration was quite apparent, the resolution was not seriously degraded. At mid-apertures the $30 \mu \mathrm{m}$ structure was resolvable and typically apertures in the range $f 11-f 4$ were used for experiments. The resolution limit for the Xenar lens was expected to be about $25 \mu \mathrm{m}$ because the CCD pixel size was roughly $8 \mu \mathrm{m}$ and the demagnification of the image was 2.8 (measured). The doublet pair, although a configuration nominally minimizing spherical aberration and optimized specifically to perform well at maximum aperture [60], did not give quite as high a maximum contrast as the Xenar lens, and aberration was considerably worse at equivalent $f$-stops. However, in mid-aperture range $(f 7-f 20)$ the $30 \mu \mathrm{m}$ structure was also resolved.

In conclusion the two lens systems described provided sufficient image quality. The resolution limits of around $25 \mu \mathrm{m}$ introduced less than a $10 \%$ correction to the smallest trap diameters of $\sim 100 \mu \mathrm{m}$. It was of course necessary to ensure that the lenses were accurately focussed, and this was checked frequently. The procedure was to form a tiny trap $\left(\sim 10^{4}\right.$ atoms $)$ at the magnetic field zero with the use of a high magnetic field gradient $\left(40 \mathrm{Gcm}^{-1}\right)$ and a low scattering rate, and focus the trap by eye with a large lens aperture to minimize the depth of field.

The imaging was calibrated by measuring the image size of an object of known dimension at the equivalent of the trap distance, and this was generally accurate to about $3 \%$. Comparison of the $z$ dimension of a trap deduced from the two orthogonal directions provided a check on the combined consistency of calibration and lens focussing, and this was typically good to $\sim 10 \%$. 


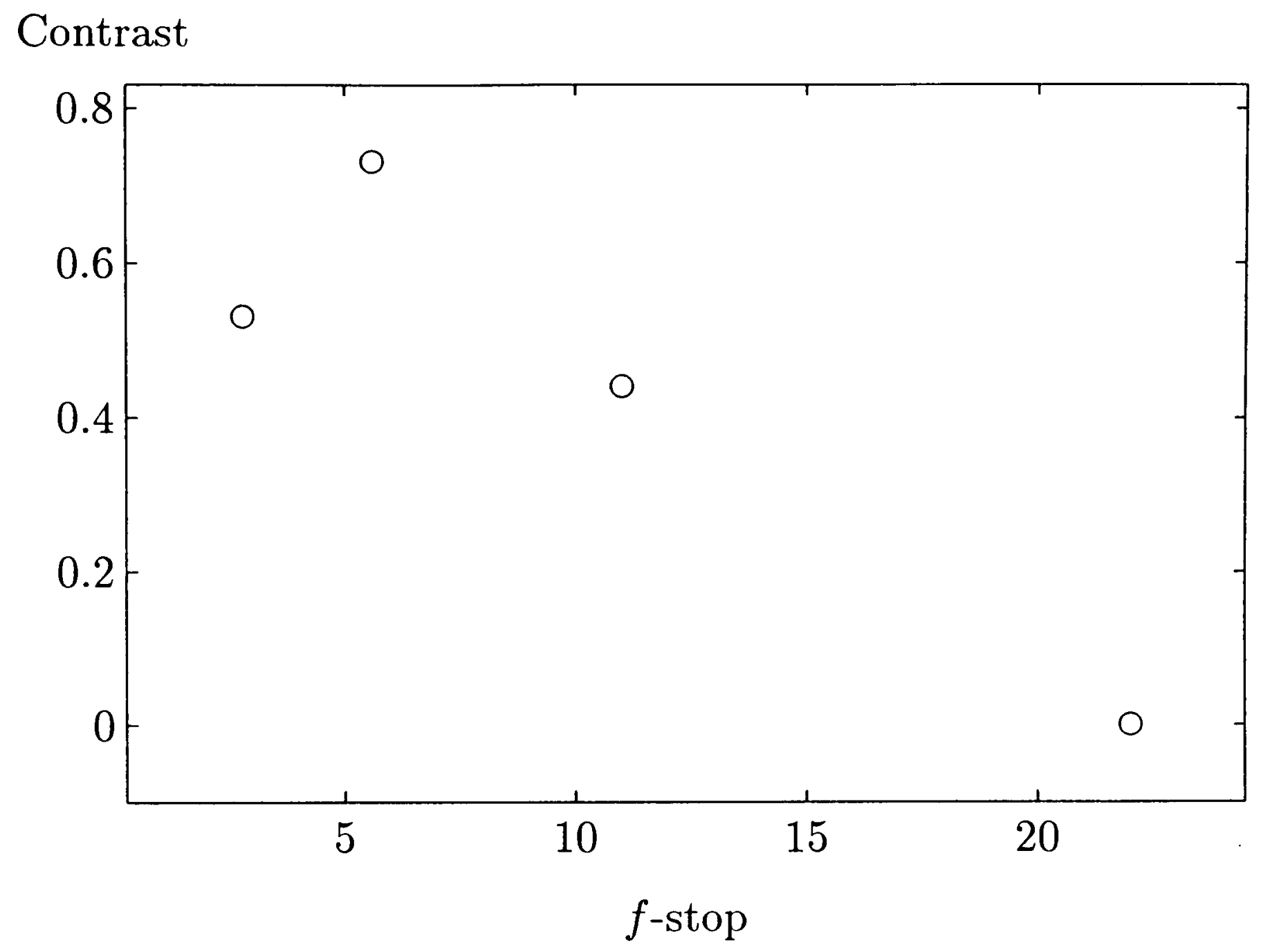

Figure 3.7: Contrast $\left(\equiv\left(I_{\max }-I_{\min }\right) /\left(I_{\max }+I_{\min }\right)\right)$ of a $30 \mu \mathrm{m}$ raster at the trap position as a function of $f$-stop of a Xenar $70 \mathrm{~mm}$ telephoto lens.

\subsubsection{Image acquisition}

In early work camera signals were recorded onto video tape and subsequently replayed in freeze-frame mode into a Data Translation Fidelity 100 frame-grabbing board to acquire images of the screen. This had a number of severe disadvantages. Acquiring images for several seconds of experiment from freeze-frame was incredibly tedious and prone to error. Resolution was limited to 40 rather $20 \mathrm{~ms}$ because freeze-frame interlaces adjacent fields. Finally, with the eventual use of the compound lenses described above the images of the trap occupied only a small fraction of the screen and it was extremely wasteful of hard disk space to record full screen images. The Fidelity 100 board was purchased on the understanding that Data Translation would provide commercial code capable of allowing the acquisition of full screens at a maximum capture rate of every other field with a 486 PC with EISA bus, but this software never became available.

During the course of the work Data Translation marketed the Vision EZ series of frame-grabbers that permit acquisition of some small fraction of the screen up to the maximum capture rate of every field. Two DT55 boards were used to digitize simultaneously the signal from each camera in real-time, vastly improving the productivity rate. With the facility to control acquisition with a trigger voltage it was possible to 
synchronize the information from each camera to within $20 \mathrm{~ms}$.

The EZ board has a number of modes of operation including a live mode, in which the camera signal is sent directly to the output screen, an acquisition mode which digitizes the camera signal onto the onboard RAM, and a freeze-frame mode which displays the contents of the onboard RAM. Digitization is at a resolution of $768 \times 512$ pixels with 256 grey scales. The ability to capture only a small area of the screen allowed the flexibility to optimize the capture speed, data size and capture duration. The acquisition rate depends proportionally on the area being captured, on the speed of the computer bus, and to a small extent on the computer processor speed. On a 486 DX50 with EISA bus the largest grid that could be captured at the maximum rate of every field was $128 \times 64$ pixels, which is about $1 / 24$ th of the total screen area and corresponds to $8 \mathrm{~K}$ of data storage. Note that in fact this corresponds to a square grid because each field contains only alternate lines of the screen. The maximum grid size allowed was $256 \times 128$ which was captured at a rate of every fourth field. Sample code was supplied with the board and this was adapted by Dr. N. H. Edwards into a program newcap that met the evolving requirements of acquisition, generation of trap profiles and non-linear fitting. A necessary feature of the program design was the writing of data to a ramdrive to avoid the $64 \mathrm{~K}$ data allocation limit imposed by standard $\mathrm{C}$. For example, with a $2 \mathrm{MB}$ drive it was possible to acquire consecutive fields for grid sizes of $128 \times 64$ continuously for more than $24 \mathrm{~s}$. The computers used for acquisition were a 486 DX50 and a Pentium P90.

\subsubsection{Measurement of temperature}

Doppler spectroscopy of a laser-cooled vapour is generally not possible because the Doppler width is typically much less than an atomic linewidth. To measure directly sub-Doppler temperature one usually looks for a ballistic effect. Early molasses experiments recorded the fraction of atoms escaping from confinement after the laser beams had been switched off for a known time, and this was related to the initial velocity distribution. This approach is very sensitive to the initial spatial distribution in molasses, and for temperatures below the Doppler limit one must account for the effect of gravity. The first measurement of sub-Doppler temperatures [55] used a time-of-flight (TOF) technique, and this has now become standard. The atoms are allowed to fall under gravity and a probe beam, located at some chosen distance below, records the ballistic expansion from which one inferes the temperature distribution.

For caesium atoms with a Maxwell-Boltzmann velocity distribution, a fall through a distance of $42 \mathrm{~cm}$ (as was used in these experiments) yields a TOF signal that is very accurately Gaussian. The initial trap shape is of no consequence. However, for lighter atoms such as sodium the TOF shape may be non-Gaussian if the distance to detection is too small or if the atoms are hot. It is instructive to understand why this is so. The problem is a simple calculation in classical mechanics. A TOF signal deviates from Gaussian because (a) the initial shape of the trap is not Gaussian, and (b) the atoms are accelerating as they pass through the probe. An assessment of (a) is straightforward. For a free-flight time $t_{0}$ a cloud with initial r.m.s. velocity $v_{0}$ will 
expand by a distance of order $2 v_{0} t_{0}=2 v_{0}(2 h / g)^{1 / 2}$ where $h$ the distance to the probe and $g$ the acceleration due to gravity. Size effects are unimportant if this expansion is much larger than the initial trap size. That is, if $\phi$ is the r.m.s. diameter of the initial distribution, if

$$
h \gg \frac{g}{2}\left(\frac{\phi}{2 v_{0}}\right)^{2}=\frac{g m}{2 k_{B} T}\left(\frac{\phi}{2}\right)^{2}
$$

where the equipartition theorem has been used in the last step. For caesium at $T=3 \mu \mathrm{K}$ and with $\phi=1 \mathrm{~mm}$ this implies $h \gg 6.5 \mathrm{~mm}$. The lowest sub-Doppler temperatures observed in sodium are $T \sim 20 \mu \mathrm{K}$ and in this instance the condition is $h \gg 1 \mathrm{~mm}$. In fact usually it would not be possible to combine the lowest MOT temperatures with a cloud size as large as $1 \mathrm{~mm}$, and thus it can be seen that size effects are nearly always negligible. By identical reasoning the effect of a finite width probe beam is also usually negligible.

Effect (b), however, can be much larger. Intuitively one would expect this to be the case for small probe distances (where the ballistic velocities are low and thus the effect of acceleration large) and for high trap temperatures (in which case the cloud is large and thus takes longer to pass through the probe). The exact shape of the TOF signal is found by expressing the initial Maxwell-Boltzmann velocity distribution $N(u)=$ $A e^{-\beta m u^{2} / 2}$ into a time distribution with the transforming equation $h=u t+g t^{2} / 2$ at constant $h$. This gives

$$
\begin{aligned}
N(t) \mathrm{d} t & =N(u) \mathrm{d} u \\
\Rightarrow N(t) & \propto \frac{1}{\sqrt{T}}\left(1+\frac{t_{0}^{2}}{t^{2}}\right) e^{-m g \beta\left(t-t_{0}\right)^{2}\left(1+t_{0} / t\right)^{2} / 2} .
\end{aligned}
$$

Fig. 3.8 shows Eq. (3.4) for caesium at two different temperatures and for two different probe distances. One can appreciate that for sodium atoms near their Doppler limit $(240 \mu \mathrm{K})$ and for probe distances $h \lesssim 10 \mathrm{~cm}$ the TOF shape will be significantly nonGaussian and one has to fit the complete form of Eq. (3.4) to the signal (as was done in [55]). However, it is clear that for caesium atoms with $h=42 \mathrm{~cm}$ the TOF shape is almost perfectly Gaussian with temperature given by

$$
\begin{aligned}
T & =\frac{m v_{0}^{2}}{k_{B}} \\
& =\frac{m g^{2} \Delta t^{2}}{k_{B}}
\end{aligned}
$$

where $\Delta t$ is the r.m.s. radius of the TOF signal.

In the experiments the TOF probe was resonant with the $F=4 \rightarrow 5^{\prime}$ transition and was retroreflected to double the intensity and to prevent pushing of the atoms. Typically the power was $1 \mathrm{~mW}$ in each direction. Light was collected with a lens of focal length $5 \mathrm{~cm}$ and diameter $4.5 \mathrm{~cm}$ and focussed onto a large area photodiode (Hamamatsu S1337-1010BR, $10 \times 10 \mathrm{~mm}$ ) whose response time of $\sim 0.5 \mathrm{~ms}$ was fast enough to resolve the lowest temperatures $(3 \mu \mathrm{K})$. The width of the probe was limited by the window size of the six-way cross and was roughly $20 \mathrm{~mm}$, implying a loss of signal 


\section{TOF signal}

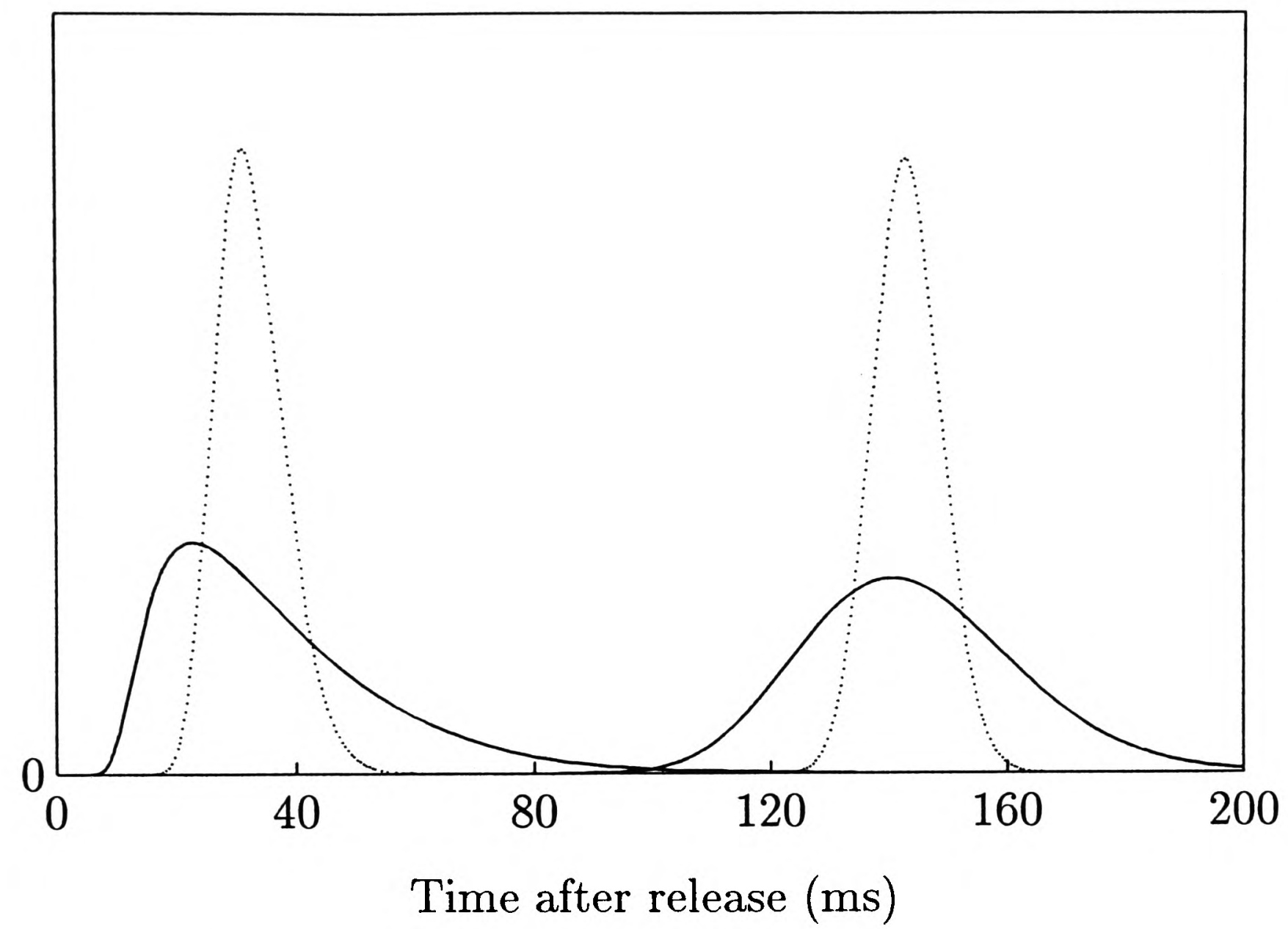

Figure 3.8: Caesium TOF signals as a function of temperature and probe distance, demonstrating Gaussian and non-Gaussian TOF shapes. The solid lines correspond to a temperature of $2 \mathrm{mK}$ and the dotted lines to $200 \mu \mathrm{K}$, with the first pair of curves detected after a free-fall distance of $0.5 \mathrm{~cm}$ and the last pair after $10 \mathrm{~cm}$.

by transverse expansion for temperatures greater than about $20 \mu \mathrm{K}$. The probe height was $2 \mathrm{~mm}$ which, for the scattering rate the probe induced, was roughly the distance travelled before the atoms were scattered into the $F=3$ dark level; no repumping light was used. One can compare the sensitivity of the TOF signal with that of detecting the trap fluorescence directly. At $10 \mu \mathrm{K}$ the spread of the cloud after falling $42 \mathrm{~cm}$ is about $1.5 \mathrm{~cm}$. Thus for a probe width of $2 \mathrm{~mm}$ a maximum of $\sim 10 \%$ of the atoms contribute to the photodiode signal. The collection solid angles for the photodiodes were roughly comparable as were the feedback resistors in the electronics. Thus overall the TOF sensitivity was about one tenth of that of the trap photodiode. Indeed to obtain a temperature for less than $10^{6}$ atoms it was necessary to filter and amplify each signal and then average over many shots. The computer control program was coded to fit the TOF signals as soon as they were recorded so that the temperature in each cycle of trapping, cooling and dropping was known before the next cycle began. 


\subsection{Computer control}

The sequence of events during experiments was coordinated with a personal computer (386 25SX) equipped with two interface boards (National Instruments) to provide digital and analogue input and output. The basic structure of the control program was designed and written in $C$ by Dr. K. P. Zetie. The use of the computer to control switching and attenuation of the light and to read several photodiode voltages has already been mentioned. Added to this list was control of magnetic coil current, providing voltage scan for the piezo of the master and synchronizing the image capture with the two frame-grabbing boards. Serial links from the computer to the two computers acquiring the image data allowed the two versions of newcap to be controlled remotely. 


\section{Chapter 4}

\section{Measurement of phase-space density in the magneto-optical trap}

\subsection{Introduction}

The original aim of the work presented in this chapter was to determine the processes which limit the spatial density in a MOT, with a view to their suppression. In pursuing this aim it became apparent that it would be necessary to measure some fundamental trap parameters, such as the spring constant and molasses temperature, in order to reduce uncertainties in interpreting the density behaviour. Performing these measurements has provided the additional benefits of both reducing current uncertainty in these fundamental properties, and providing a test of the model presented in chapter 2. It has thus enabled a description of the MOT which embraces a wide range of its behaviour. With the widespread use of the MOT as a convenient source of cold, dense atoms an empirical understanding between experimental parameters and phase-space density is of general interest.

During the course of the work it was necessary to consider the possibility that the spatial density in the MOT is unexpectedly sensitive to the quality of the trap alignment and the spatial profile and polarization of the trapping beams. For this reason we took the opportunity to complement the work completed in Oxford with an independent study of MOT densities on two traps at the Ecole Normale Supérieure, Paris ${ }^{1}$, undertaken by Dr. Andrew Steane and co-workers. The results of this combined survey are reported in [83]. In the following description of the work the emphasis is necessarily placed upon that undertaken in Oxford; however, where appropriate, complementary results from Paris are also presented. It will be made clear where this is the case.

The chapter is organized in the following way. In Sec. 4.2 relevant features of the experimental setup in Oxford are summarized, and are compared to those of the two Paris experiments. To deduce the number of atoms trapped in the MOT from the fluorescence they emit it is necessary to know the distribution of population among

\footnotetext{
${ }^{1}$ Laboratoire Kastler Brossel, Unité de recherche de l'Ecole Normale Supérieure et de l'Université Pierre et Marie Curie, associée au CNRS, 24 rue Lhomond, F-75231 Paris CEDEX 05, France.
} 
the ground state sublevels. A measurement of this distribution is described in Sec. 4.3. In Sec. 4.4 measurements of the temperature in $\sigma^{+}-\sigma^{-}$optical molasses (equal to that of a low density MOT) are presented, confirming those reported by Drewsen et al. [25]. In Sec. 4.5 our own measurements of the confining force at the trap centre are presented, and with the technique employed it is deduced that multiple scattering limits the density before it heats the cloud. Sec. 4.6 is concerned with the two-component density regime. It is demonstrated that the trapped cloud can easily extend beyond the point at which the confining force breaks down spatially, and measurements of the capture range for strong confinement are reported, showing reasonable agreement with the simple theory described in chapter 2. The behaviour of the cloud as it extends beyond the capture range is investigated in both real and momentum space. Sec. 4.7 demonstrates how the technique of transiently reducing the scattering rate in the MOT, in addition to allowing access to densities at low saturation of the atomic transition, also permits a determination of the position damping time. The variation of this time with detuning is presented and compared to a previous measurement [25]. The density measurements themselves are described and presented in Sec. 4.8 and compared to previous measurements. Finally phase-space density is discussed in Sec. 4.9.

\subsection{Experimental arrangements}

Unless otherwise stated, all commentary in this chapter refers to the experiment in Oxford; the two traps in Paris shall be specifically labelled traps P1 and P2 to avoid confusion.

For comparison with the traps P1 and P2 some features of the experimental setup described in chapter 3 are worth summarizing. The trap in Oxford was formed at the centre of a large stainless steel vacuum chamber pumped by a diffusion pump. The chamber windows were anti-reflection coated and were of high optical quality. The trapping light was spatially filtered using single-mode optical fibres. Two horizontal trapping beams were produced with light split from one fibre, and light from a second fibre produced a vertical beam, with each beam retroreflected back upon itself from outside the vacuum chamber to an angular alignment of better than $5 \times 10^{-4}$ radians. With this optical arrangement care was taken to maintain as much as possible the optical quality of the spatially filtered beams. With the use of a minimum number of optics, all of high quality, it was found that deviations from perfect Gaussian profiles after a single pass through the chamber typically consisted of $5-15 \%$ fluctuations in the intensity, with spatial periods ranging up to a few hundred micrometres. Three methods could be employed to load the trap: vapour loading, "semi" vapour loading and beam loading. In combination these enabled steady-state trap numbers to be varied in the range roughly $10^{4}-10^{8}$ atoms, with trap lifetimes varying between $0.25-5 \mathrm{~s}$.

Trap P1 was formed in a glass cell pumped by a $25 \mathrm{ls}^{-1}$ ion pump, which produced a pressure less than $3 \times 10^{-9}$ Torr. The trap was loaded from a second magneto-optical trap about $60 \mathrm{~cm}$ above it, housed in another chamber of the same vacuum system. The upper trap collected atoms from a caesium vapour at a pressure $\sim 3 \times 10^{-8}$ Torr for $2 \mathrm{~s}$. The collected atoms were then cooled to $3 \mu \mathrm{K}$ and allowed to drop through a 
tube down to the lower trap, which caught them. About $20 \%$ of the atoms collected in the upper trap could be transferred to the lower trap in this way. The lower trap (trap P1) was formed using retroreflected laser beams; the walls of the glass cell were not anti-reflection coated and were not of good optical quality, neither were the beams spatially filtered.

Trap P2 was formed in a glass cell pumped by a $25 \mathrm{ls}^{-1}$ ion pump. The cell was roughly spherical with 6 flat regions (uncoated) to form windows for the trapping beams. The trapping light was spatially filtered and divided into 6 independent beams before being passed into the cell. The background gas in the cell was predominantly caesium at a pressure of a few $10^{-8}$ Torr; the trap captured slow atoms from this vapour.

In all three experiments calibrated CCD video cameras were used to observe the fluorescence of the trapped cloud of atoms, and deduce the density (see Sec. 4.8). In Oxford the trap was observed simultaneously with two cameras positioned orthogonally and at $45^{\circ}$ to the horizontal trapping beams, with a resolution of about $25 \mu \mathrm{m}$. Trap $\mathrm{P} 1$ was observed by a single camera looking along a direction at $20^{\circ}$ to the $y$-axis (and occasionally at right angles to this); trap P2 was observed along the $(0,1,1)$ diagonal. The resolution of the optics for both traps P1 and P2 was $12 \mu \mathrm{m}$, and was measured by imaging a series of pinholes of various sizes.

In all experiments light was provided by diode lasers locked to the correct detuning from the caesium resonance using saturated absorption spectroscopy. Each trap had light near-resonant with the $F=4 \rightarrow 5^{\prime}$ transition, and "repumping" light on the $F=3 \rightarrow 4^{\prime}$ transition to remove atoms from the lower hyperfine level of the ground state. Since time-dependent measurements of the density could extend over several seconds, it was important to ensure that the laser detunings were stable to within a $\mathrm{MHz}$ on this timescale.

\subsection{The fluorescent emission}

To calculate the trap number from the emitted fluorescence it is necessary to make an estimate of the average scattering rate per atom. For a two-level atom illuminated by a single travelling wave of Rabi frequency $\Omega$ and detuning $\delta$ the scattered power is [57]:

$$
P=\hbar \omega_{L} \frac{\Gamma}{2} \frac{\Omega^{2} / 2}{\Omega^{2} / 2+\Gamma^{2} / 4+\delta^{2}}
$$

In the MOT, however, the Rabi frequency and electric field polarization are both complicated functions of position, and the presence of magnetic sublevels in the alkalis means that one must in addition consider the existence of position-dependent transition rates:

$$
P=\frac{\hbar \omega_{L}}{N} \frac{\Gamma}{2} \int \sum_{M M^{\prime}} n_{M}(\mathbf{r}) \frac{C_{M M^{\prime}}^{2} \Omega_{t o t}^{2}(\mathbf{r}) / 2}{\delta^{2}+\Gamma^{2} / 4+C_{M M^{\prime}}^{2} \Omega_{t o t}^{2}(\mathbf{r}) / 2} \mathrm{~d}^{3} \mathrm{r}
$$

where $C_{M M^{\prime}}$ is the Clebsch-Gordan coefficient connecting sublevels $M, M^{\prime}$ and $n_{M}(\mathbf{r})$ is the spatial density of atoms in the sublevel $M$. A standard approach to handle this 
situation [54] is to assume that the scattering rate per atom can be approximated as

$$
P \simeq \hbar \omega_{L} \frac{\Gamma}{2} \frac{C_{1}^{2} \Omega_{t o t}^{2} / 2}{\delta^{2}+\Gamma^{2} / 4+C_{2}^{2} \Omega_{t o t}^{2} / 2}
$$

where $\Omega_{\text {tot }}^{2}$ is taken to be $6 \Omega^{2}$, and $C_{1}$ and $C_{2}$ are "average Clebsch-Gordan coefficients". One might expects $C_{1}$ and $C_{2}$ to be of the same order of magnitude, though not necessarily equal.

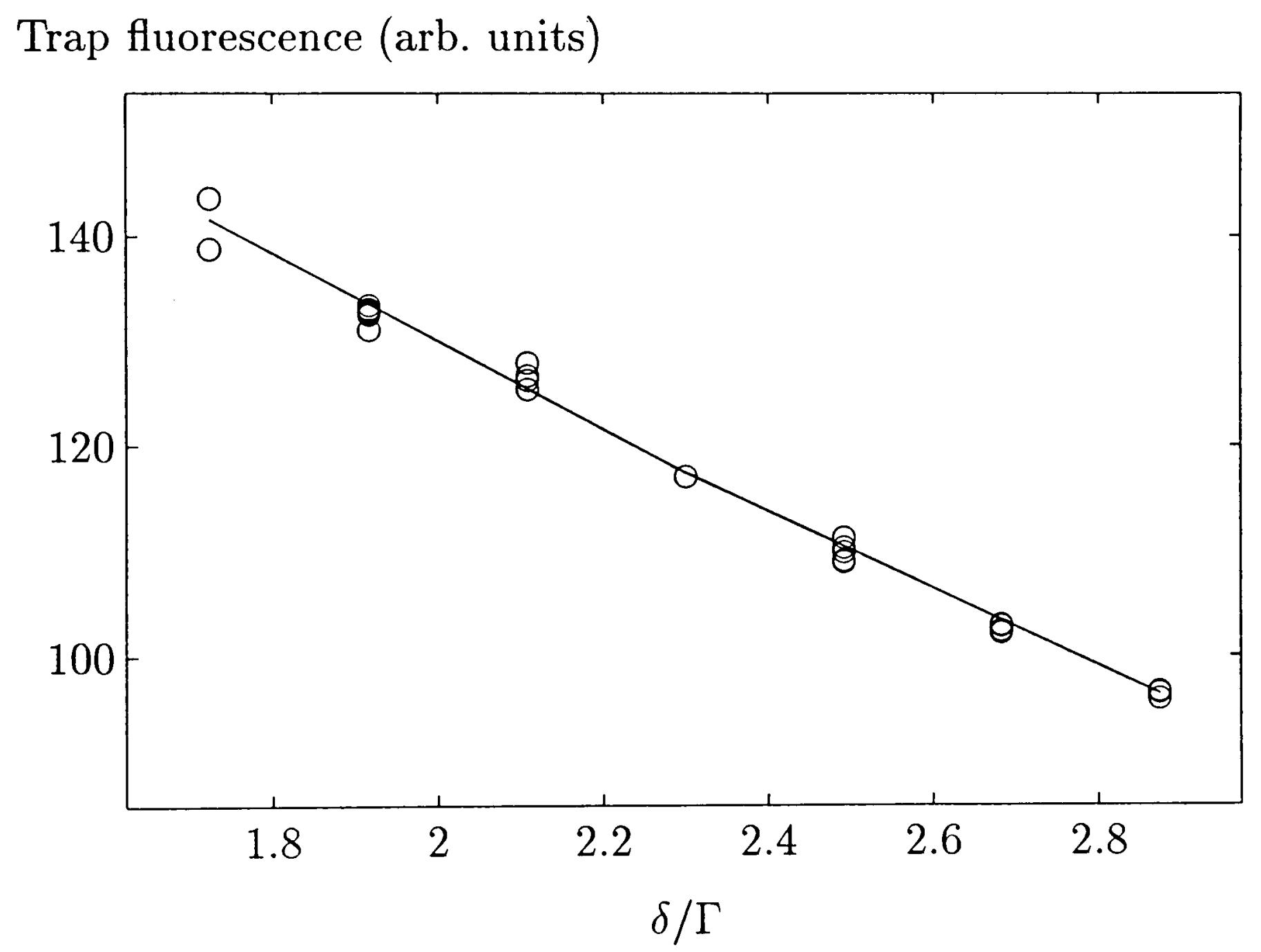

Figure 4.1: Variation in trap fluorescence with detuning for a fixed number of atoms. The solid line is a least squares fit of Eq. (4.3) and yields $C_{2} \Omega=4 \Gamma$, with an uncertainty of about $5 \%$.

To measure $C_{2}$ the fluorescent power scattered by a fixed number of atoms in the MOT was measured with a photodiode as the detuning was switched rapidly between starting and final values. A fit of Eq. (4.3) to the variation with $\delta$ then allows $C_{2}$ to be deduced, assuming that $\Omega_{\text {tot }}$ is known. To make an accurate measurement of $\Omega_{\text {tot }}$ account was taken of the effects on the beam intensity such as loss during the retroreflection, convergence of the laser beams, and absorption by the trapped cloud. To ensure that the optical thickness of the cloud remained reasonably constant (and thus minimizing the absorption effect that the cloud itself had upon the average intensity) the change in detuning was kept fairly small, typically between $2 \Gamma$ and $3 \Gamma$. An example of the variation in fluorescence observed is shown in Fig. 4.1. 
A possible approach to determining $C_{1}$ is to measure the change in fluorescent power incident on the photodiode as all but one of the trapping beams are rapidly switched off, all other conditions remaining unchanged. The single remaining (circularly polarized) beam quickly optically pumps the atoms into the stretched state $|F=4, M=4\rangle$, and one measures the scattered power, now given by Eq. (4.1), before the atoms are pushed away by this beam. Taking into account the factor six change in the average light intensity, and the value of $C_{2}$ already measured, the ratio of the photodiode signals before and after cutting the beams allows $C_{1}$ to be deduced. The following procedure was adopted. The MOT was first fully loaded. The trapping beams were then extinguished rapidly with the AOMs (in less than a microsecond), and a mechanical shutter positioned in the retroreflecting arm of the vertical trapping direction was closed. The AOM controlling the vertical light was then switched back on to induce fluorescence from a single beam. To enable the preferred quantization axis to lie along the vertical direction it was necessary to turn off the magnetic field gradient before the optical pumping stage. It was found that in order to be able to time-resolve the decay of the fluorescence signal under the pushing effect of the single beam, the response time of the photodiode (RS $65199541.3 \mathrm{~mm}^{2}$ ) had to be less than about $100 \mu \mathrm{s}$. The reduced gain this incurred meant that a measurement was only possible at high saturation of the atomic transition where the fluorescence signal was large.

The results of both experiments are shown in Fig. 4.2, and represent measurements made over a period of several months, thus encompassing a number of trap realignments. All measurements were made with Rabi frequencies lying between $\Gamma$ and $2 \Gamma$, and detunings between $2 \Gamma$ and $3 \Gamma$. The mean of all the measurements of $C_{2}^{2}$ gave $C_{2}^{2}=0.73 \pm 0.14$, with the most likely cause of the scatter being the combination of fluctuations in the intensity of the beam intensity profiles with the different trap alignments. $C_{1}$ and $C_{2}$ were found to be equal within a combined uncertainty that is almost entirely due to that in determining $C_{2}^{2}: C_{1}^{2} / C_{2}^{2}=1 \pm 0.2$.

One might suppose that because the light in the three-dimensional interference pattern of a MOT has all kinds of polarizations at different places, so the value of $C_{1}^{2}$ and $C_{2}^{2}$ should be similar to the average for all the transitions and all the polarizations, i.e. for a $4-5^{\prime}$ transition $C_{1}^{2} \simeq C_{2}^{2} \simeq \sqrt{11 /(3 \times 9)} \simeq 0.4$. These results show that this reasoning is not valid. The measured values indicate that an atom spends more time in a magnetic sublevel that interacts strongly with the light, i.e. the coupling between the atoms and the radiation field is stronger than a simple average over all possible coupling strengths, thereby raising the overall fluorescent scattering rate. This optical pumping process is at the heart of the Sisyphus effect which is predominantly responsible for the friction force in the MOT. In addition, optical potential wells tend to cause atoms to spend more time in regions where their interaction with the light is the strongest, since the light shift of the ground state is most negative for the atomic state having the greatest coupling with the light (for a laser field detuned red of the atomic resonance). Therefore the measurements may be interpreted as experimental evidence that one or both of these physical processes is indeed present.

A quantum Monte-Carlo calculation for $F=4 \rightarrow 5^{\prime}$ in a three-dimensional $\sigma^{+}-\sigma^{-}$ optical molasses has yielded an excited state fraction consistent with $C_{1}^{2}=0.9 \pm 0.1$, at 


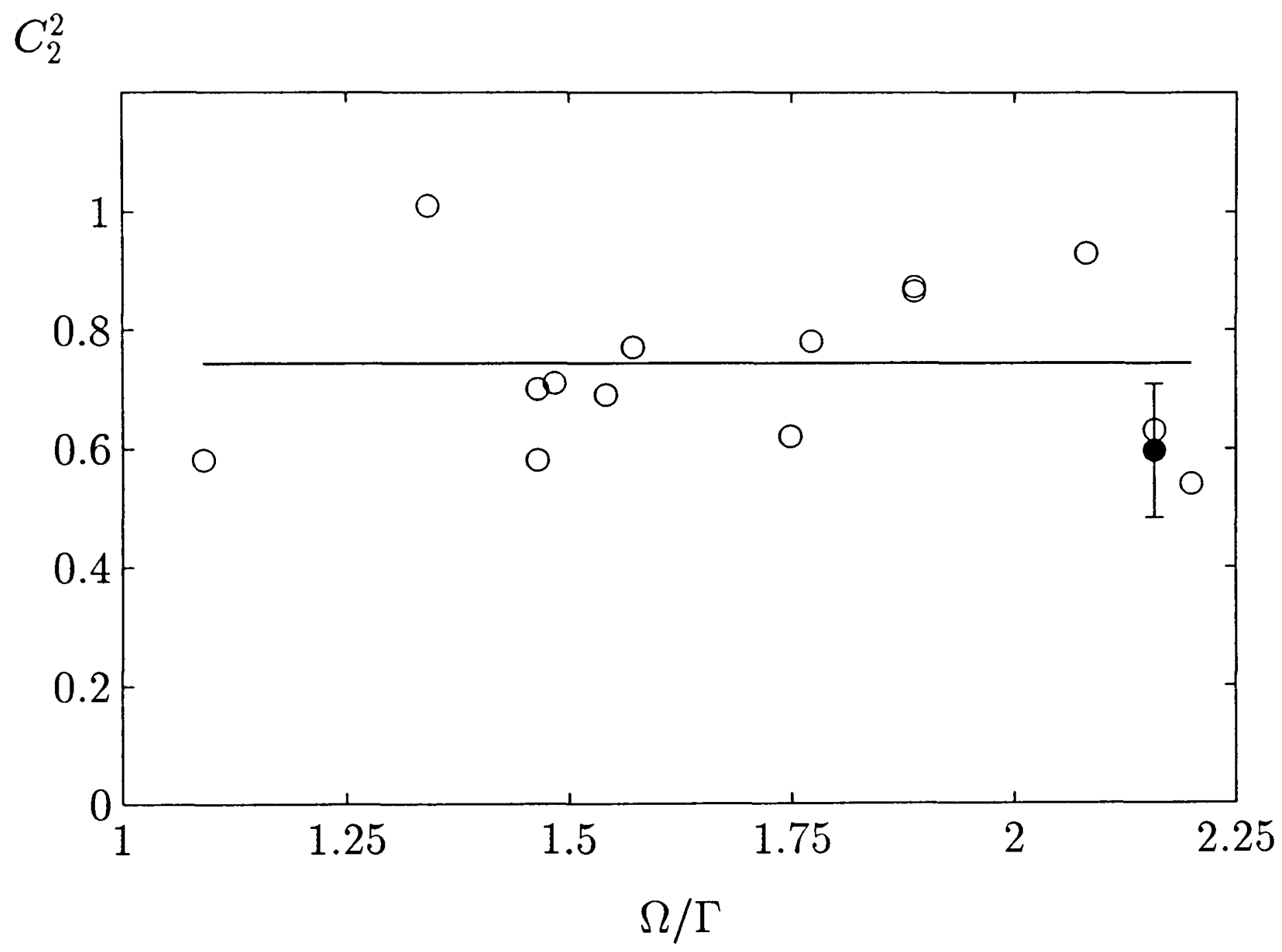

Figure 4.2: Squares of the average Clebsch-Gordan coefficients, deduced by the methods described in the text. The open circles are measurements of $C_{2}^{2}$, and were taken over a six month period with numerous different trap alignments and two different trapping beam geometries. The main cause of the scatter is probably spatial fluctuation in Rabi frequency at the trap position arising from 5-20\% spatial inhomogenities in the trapping beam profiles and their retroreflections. The solid line marks the average $C_{2}^{2}=0.73$, and the standard deviation is 0.14 which is larger than a $10 \%$ systematic uncertainty arising from the estimate of $\Omega_{t o t}^{2}$ at the trap centre. The filled circle shows the average of several measurements of $C_{1}^{2}$, with an error bar that is mainly due to the uncertainty in determining $C_{2}^{2}$. The value of $C_{2}^{2}$ at the same $\Omega / \Gamma$ was measured in the same experimental run; by comparing the ratio of signals the uncertainty in concluding that $C_{1}^{2} / C_{2}^{2}=1$ is relatively unaffected by the uncertainty in knowing $\Omega$. 
$\delta=5 \Gamma, \Omega=0.71 \Gamma[9]$. Note, however, that these parameter values are different from those used in the experiment, and that in the calculation the phase differences between the three standing waves were kept constant whilst in the experiment they fluctuate.

In subsequent calculations of the trap number the values $C_{1}^{2}=C_{2}^{2}=0.7 \pm 0.2$ have been used. The discrepancy with the use of $C_{1}^{2}=C_{2}^{2}=0.4$ is generally less than $50 \%$. Wallace et al. [87] calculated the excited state fraction by assuming an equal distribution among the sublevels, and compared it to that measured from the photoionization rate. Agreement was found to within $20 \%$.

\subsection{Temperature measurements}

With a setup in Oxford similar to that described above, it was previously verified that there is a heating of the trap above the molasses temperature in the multiple scattering regime by an amount proportional to $N^{1 / 3}$, in agreement with the initial observation by Drewsen et al. [25]. The experiment has been described in detail in [16] and is summarized in [17]. The scaling of the excess temperature with detuning and intensity was found to be roughly the same as that reported in [25], but the molasses temperature, determined by the temperature in the $N \rightarrow 0$ limit, was not in good agreement. The reason for this discrepancy was suggested to be the sensitivity of the temperature in three dimensions to the relative phases of the circularly polarized beams. This has been observed theoretically in a semi-classical calculation by Wallace et al. [89].

The reasons for wanting to measure accurately the temperature in caesium $\sigma^{+}-\sigma^{-}$ molasses were cited in chapter 2. There are two ways to prepare a molasses. The simplest is to rely on those atoms in the background vapour with velocities small enough to be slowed in the intersection volume of the laser beams. Typically this is a total number less than that attainable in a MOT [25]. This method was not employed because (a) despite the large trap-TOF probe separation of about $40 \mathrm{~cm}$, a source volume of $\sim 1 \mathrm{~cm}^{3}$ will impose a geometrical broadening on the TOF signal of width $\sim 5 \mu \mathrm{K}$ and with a shape that is somewhat uncertain; this is larger than the lowest molasses temperatures of a few microkelvin, (b) a source volume of this size combined with the long free-flight time causes a substantial fraction of the atoms to fall outside the $2 \mathrm{~cm}$ wide probe beam for temperatures greater than about $20 \mu \mathrm{K}$, and (c) it was intended to measure the molasses temperature as a function of the trapping intensity, but the intensity over the intersection volume is (generally) not uniform. The effects of (a) and (c) can be reduced by measuring the temperature of a thin slice of the molasses only [73], but they are avoided completely by releasing atoms into molasses from a MOT, i.e. loading a MOT and switching the magnetic field gradient off. This gives a small initial size which is comparable to the trap volume $\left(\ll 1 \mathrm{~mm}^{3}\right)$ and whose contribution to the final spreading is negligible $\left(\ll 1 \mu \mathrm{K}\right.$ for a trap size of $1 \mathrm{~mm}^{3}$ falling through $40 \mathrm{~cm}$ ), and the light intensity is uniform over the cloud. Further, the number of atoms in the molasses is as large as that in the MOT.

However, releasing directly from a MOT has the disadvantage for measuring the molasses temperature (i.e. the temperature in the low density limit) that the ensuing 
molasses is of high density. One must wait for the cloud to expand under the repulsive forces from atom-atom interactions and from diffusion through molasses in order for the excess heating from multiple scattering to subside. This typically takes up to $50 \mathrm{~ms}$ [25]. Unfortunately we found that on this timescale it was difficult to get the cloud to expand uniformly and not to be pushed to one side by the imbalance in the trapping beam intensity, which had a large effect in the absence of the confining magnetic field. Thus the problems of geometrical broadening and uncertainty in the cooling intensity are reincurred, made worse by the fact that the effect of the imbalance, and thus the intensity region into which the cloud is pushed, depends on the intensity and detuning, i.e. the very parameters against which one wishes to measure the molasses temperature. Indeed it was observed that as the time for expansion was allowed to increase to much longer than $50 \mathrm{~ms}$ the measured temperature generally continued to decrease, suggesting cooling in a low intensity outer region of the intersection volume. The problem was avoided by working with a sufficiently small number of atoms to avoid heating from multiple scattering. By loading from the thermal atomic beam it was possible to trap less than $10^{6}$ atoms and, with electronic low-pass filtering and amplification, to retain a reasonable TOF signal. According to both [25] and [17] the excess heating from multiple scattering with this few atoms is less than about $1 \mu \mathrm{K}$. This was confirmed by varying the trap number between $8 \times 10^{4}$ and $3 \times 10^{6}$ atoms and measuring the trap temperature at the fixed parameters $\delta \approx 6 \Gamma, \Omega^{2} / \delta \Gamma \approx 0.1$. Between $4 \times 10^{5}$ and $3 \times 10^{6}$ atoms the temperature was observed to change by $\lesssim 10 \%$, and below $4 \times 10^{5}$ atoms by much less than this. It was thus not necessary to give time for cloud expansion, and pushing of the cloud centre was thus avoided. Therefore, this approach removed any variation in the position the cloud during cooling. In what follows the trap number was just less than $10^{6}$.

The following TOF sequence was adopted. The trap was loaded with $\delta=2 \Gamma$, $\Omega=1.5 \Gamma$ and $b=10 \mathrm{Gcm}^{-1}$. The parameters $\{\delta, \Omega\}$ were then switched to the required values. After a wait of a few milliseconds the magnetic field gradient was switched off, followed by the light about $10 \mathrm{~ms}$ later. The results for all $\{\delta, \Omega\}$ are shown in Fig. 4.3(a). Each point represents the average of between one and ten shots, depending on the strength of the TOF signal; this tended to be weak at $\delta<4 \Gamma$, and at the lowest intensities for all detunings. We notice first that the temperature at low detunings is larger than the linear dependence on $\Omega^{2} / \delta \Gamma$, as predicted to occur specifically in $\sigma^{+}-\sigma^{-}$ molasses by Mølmer [63], and as previously observed in rubidium [89] and caesium [25]. The data for $\delta>4 \Gamma$ and $T<35 \mu \mathrm{K}$ is replotted in Fig. 4.3(b). For each detuning the temperature is clearly observed to pass through a minimum. When $T<10 \mu \mathrm{K}$, a linear fit to the data for $\Omega^{2} / \delta \Gamma>0.025$ yields [see Eq. (2.9)]

$$
C_{0} / k_{B}=1.9 \pm 0.5 \mu \mathrm{K}, C_{\sigma}=0.24 \pm 0.05 \quad\left(\Omega^{2} / \delta \Gamma>0.025, T<10 \mu \mathrm{K}\right)
$$

in agreement with the results of [25] for this temperature range. However, in addition, a slight departure from a linear dependence on the light shift is observed. Forcing a straight line fit through all the data for $T<35 \mu \mathrm{K}$ gives

$$
C_{0} / k_{B}=1 \pm 0.5 \mu \mathrm{K}, C_{\sigma}=0.28 \pm 0.05 \quad\left(\Omega^{2} / \delta \Gamma>0.025, T<30 \mu \mathrm{K}\right)
$$


Temperature, $T(\mu \mathrm{K})$

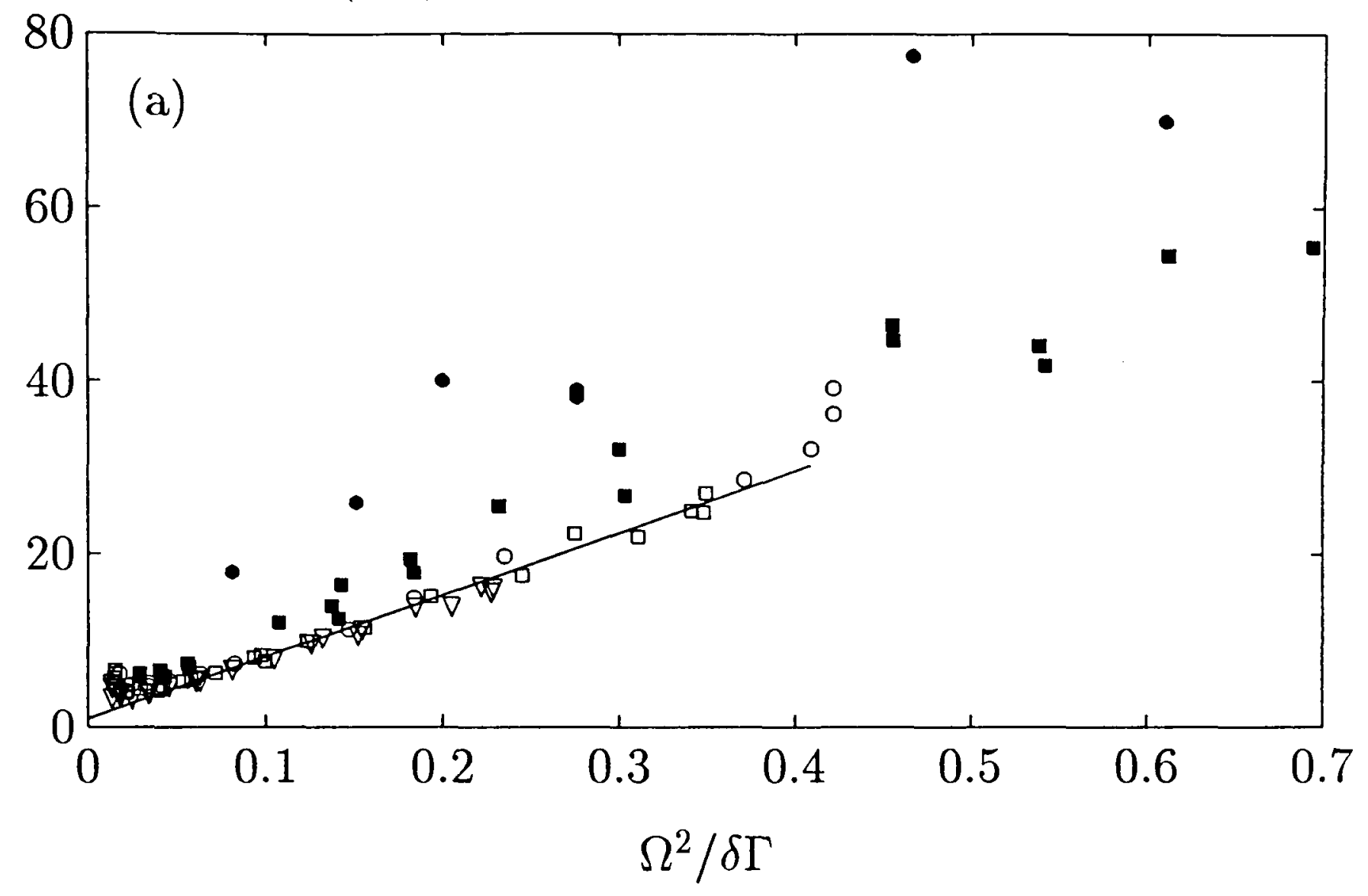

Temperature, $T(\mu \mathrm{K})$

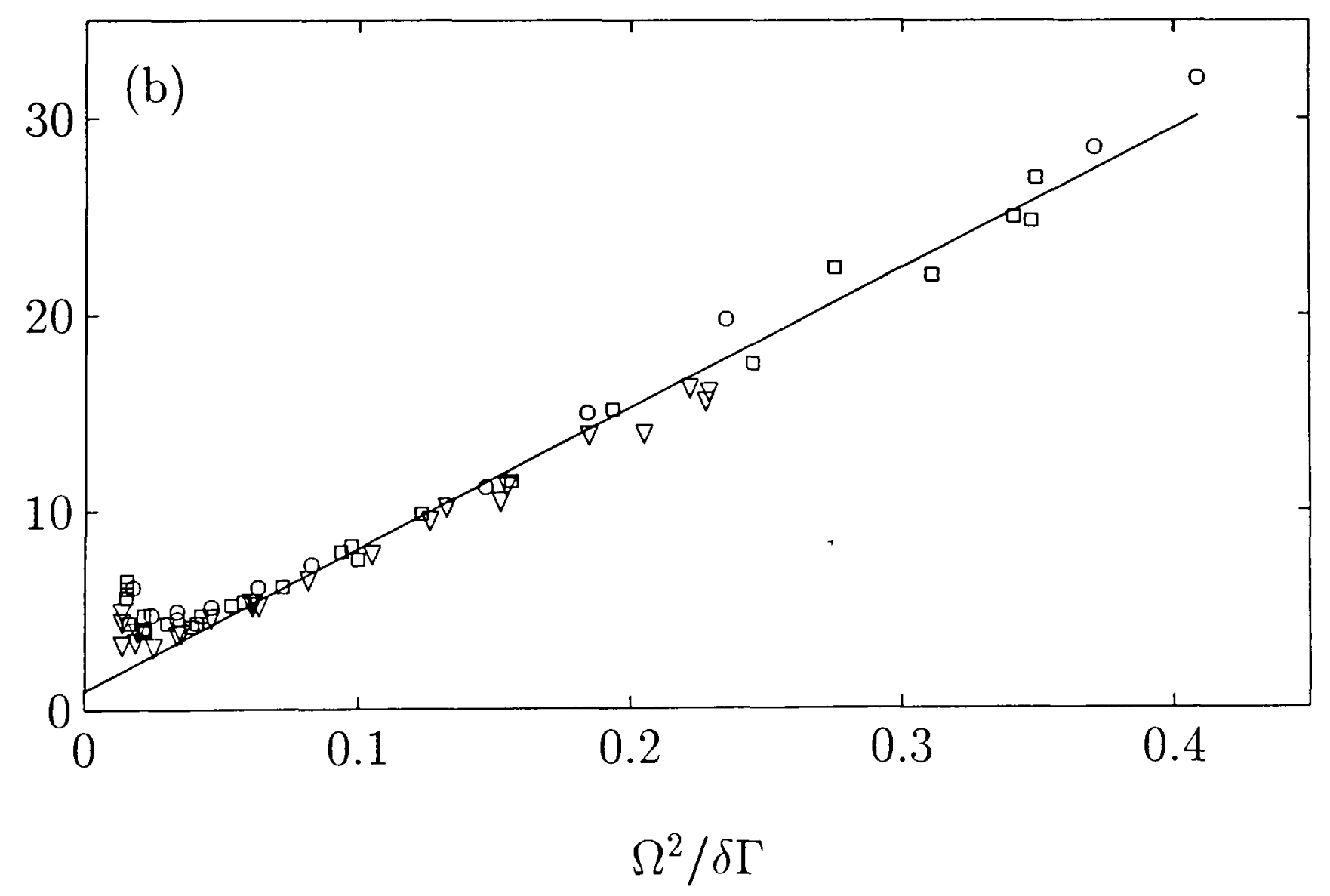

Figure 4.3: Temperature measurements on atoms released from a $\sigma^{+}-\sigma^{-}$optical molasses formed from three mutually perpendicular beam pairs. The complete data set is shown in (a), and the data for $\delta>4 \Gamma, T<35 \mu \mathrm{K}$ repeated in (b). The symbol shape indicates the detuning as follows: $\delta / \Gamma=$ $1.9(\bullet), 3.8(\mathbf{\square}), 5.7(0), 7.6(\square), 11.4(\nabla)$. 
The uncertainty in $C_{\sigma}$ is almost entirely due to an estimated $15 \%$ uncertainty in the average intensity of the laser field at the position of the trapped cloud.

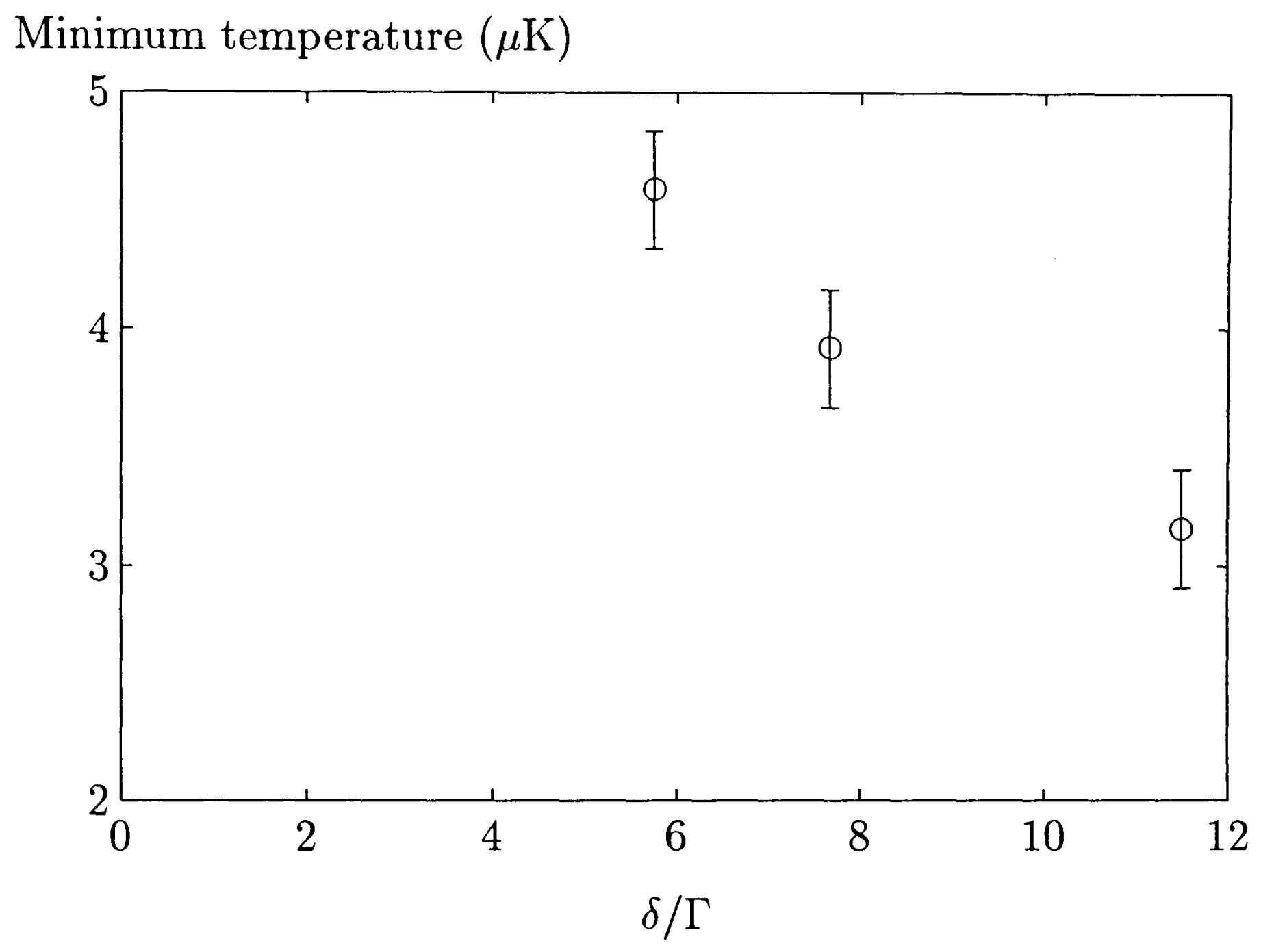

Figure 4.4: Minimum temperature as a function of detuning, to be compared to the data of Drewsen et al. [25]. The error bars have been estimated from the scatter on the temperature measurements near the minima.

The temperature minima at low saturation occur at $\Omega^{2} / \delta \Gamma \approx 0.025$, in quantitative agreement with the breakdown point observed by Drewsen et al. [25], but a factor two smaller than that observed by Wallace et al. [89] (for both ${ }^{85} \mathrm{Rb}$ and ${ }^{87} \mathrm{Rb}$ ). According to the quantum formulation by Castin et al. [10] the temperature in molasses in the limit of large detuning is a function of the light shift and the angular momentum only. However Fig. 4.4 shows that the minimum temperature was measured to decrease slightly with increasing detuning; this data is in excellent quantitative agreement with figure 7 of [25]. A shifting of the minimum to lower light shifts with higher detuning is just resolvable above the $0.5 \mu \mathrm{K}$ scatter in the data.

\subsection{Measurement of $\kappa$}

It was important to be certain that the magnitude of the spring constant was comparable to previous reports, not reduced by imperfections in the optical setup, such as 
the use of only three rather than six independent trapping beams. The information that has been obtained has helped to reduce the uncertainty illustrated by Table 2.1, and the study has been useful in understanding the difficulties in measuring the spatial properties of the MOT in the temperature-limited regime.

The chosen method was to measure the radius of a temperature-limited trap and deduce $\kappa$ from Eq. (2.5), knowing the temperature. The difficulty with this procedure is that it must be ensured that the conditions are such that the trap is indeed temperaturelimited, i.e. that the trap dimensions are independent of the trap number. A simple way to be sure of this is to measure the size of the cloud as the trap loads, and use the radius in the limit of small number to deduce $\kappa$; this has the advantage that one can see exactly how constant with number the dimensions are. This idea was implemented with the following techniques. The basic procedure was to switch on the magnetic field coils and acquire images of the subsequent loading with the fast frame-grabber. However, one sees immediately from the loading equation (2.34) that it is impossible to detect fewer than a fraction $\Delta t \Gamma$ of the final steady-state number with an acquisition time resolution $\Delta t$ and for a trap lifetime $\Gamma^{-1}$. Thus for a typical loading from a background vapour of $10^{7}$ atoms with $\Gamma^{-1}=1 \mathrm{~s}$, measurement would be limited to greater than $10^{5}$ atoms for $\Delta t=20 \mathrm{~ms}$, the time difference between consecutive video fields. To extend detection to a much smaller number it is necessary to reduce the final number in the trap. This was achieved by loading the trap from a very low background pressure, attained by shuttering the thermal loading beam completely, and thus permitting capture only from the small residual vapour inside the vacuum chamber. In this way tiny traps of $\sim 10^{4}$ atoms were obtained, and by monitoring their loading it was possible to measure trap dimensions for as few as $10^{3}$ atoms. In addition, it was straightforward to combine the information from the loading curves for these tiny traps with that obtained for larger traps, loaded under the same trapping conditions but from the unshuttered thermal beam. By varying the oven temperature, and thus the beam flux, it was possible in this way to measure the variation of trap volume with number over the range $10^{3}-10^{6}$ atoms, and thus establish the boundary between the temperature-limited and multiple scattering regimes.

A typical variation of the trap radius with number for a high trapping intensity is shown in Fig. 4.5, and clearly demonstrates the transition from temperature-limited to multiple scattering-limited behaviour. It is noticeable that the change from constant to $r \sim N^{1 / 3}$ variation [c.f. Eq. (2.18)] is gradual rather than sharp. An important observation was that at lower beam intensities the trap was never unambiguously observed to be temperature-limited. That is, the trap radius began to increase as soon as it was detected, so it was not possible to be sure that the radius would not be smaller with less than $10^{3}$ atoms. This is evidence for the prediction in Sec. 2.2.4 that the boundary between the two regimes moves to lower number with decreasing intensity. In this instance a measurement of the smallest detected radius would only give a lower limit for $\kappa$ rather than its absolute value. The transition between the regimes was observed equally clearly with other parameter values listed for the Oxford trap in Table 4.1. It was noticed that at the lower field gradient the radius remained constant with number to larger numbers, but that at the lower detuning there was no discernible difference in 


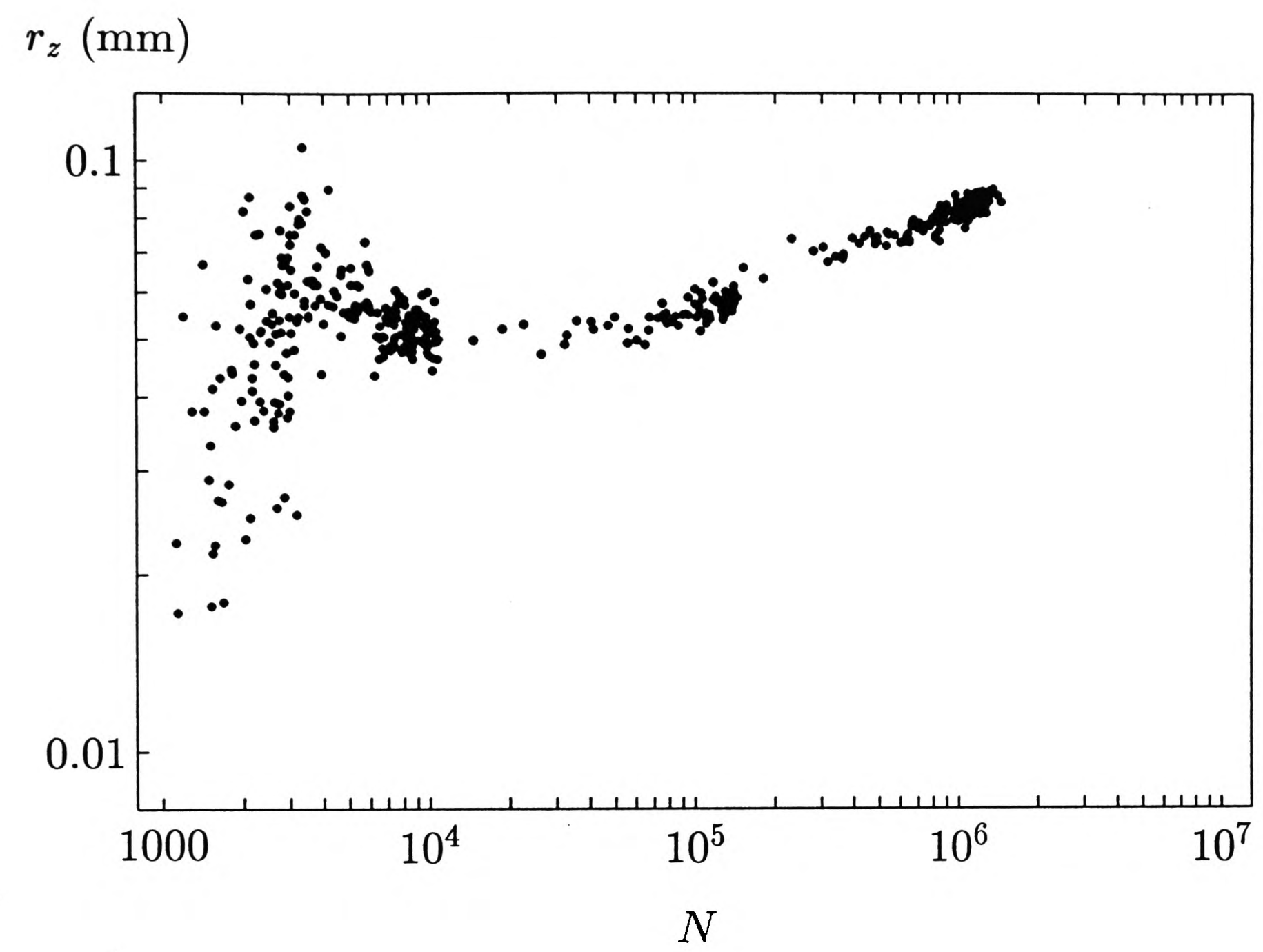

Figure 4.5: Variation of r.m.s. radius along the $z$-axis as a function of trap number, with the trapping parameters $\delta=3.4 \Gamma, \Omega^{2}=5.2 \Gamma^{2}$ and $b=10 \mathrm{Gcm}^{-1}$. The data shown is the combination of three loading sequences with different final steady-state numbers, and each point represents the image acquired from one field of the CCD signal.

the transition position. These observations are also in agreement with the variations suggested in Sec. 2.2.4.

The last column of the first four rows of Table 4.1 list the results obtained in Oxford by converting the constant radii into values of $\kappa$. It has been assumed that the agreement of the molasses temperature measurements with those of Drewsen et al. [25] is good evidence for the reproducibility of Fig. 4.3, and thus the temperatures were not measured simultaneously with the radii (in fact it would have been exceedingly difficult to have obtained a TOF signal for only $10^{3}$ atoms, spread temporally by their relatively high temperature of $\sim 65-80 \mu \mathrm{K}$ at the parameter values indicated, and have simultaneously retained the temporal resolution in the detection required to resolve the signal). The spread in the values of $\kappa_{0}$ of a factor $\sim 2$ is attributable to the uncertainty in the trap radius at small number (low intensity CCD images leading to the scatter observed in Fig. 4.5), and to uncertainty in the molasses temperature at the necessarily high light shifts. Also included in Table 4.1 are measurements taken in Paris; these used much lower intensities and generally did not reveal a temperature-limited trap, which 
Table 4.1: Information relevant to deducing the spring constant $\kappa$. In the final columns, the spring constant is calculated by assuming the trap is in the temperature limited regime and by taking the temperature from Fig. 2.1. For the measurements from Paris when the trap was obviously not in the temperture limited regime no value is given, and when the trap was close to but not quite temperaturelimited the value is given in brackets.

\begin{tabular}{|c|c|c|c|c|c|c|c|}
\hline Trap & $\Omega^{2} / \Gamma^{2}$ & $\delta / \Gamma$ & $\begin{array}{l}\mathrm{d} B / \mathrm{d} z \\
\mathrm{Gcm}^{-1}\end{array}$ & $N / 10^{5}$ & $\begin{array}{l}r \\
\mu \mathrm{m}\end{array}$ & $\begin{array}{l}\kappa \\
\left(10^{-19}\right.\end{array}$ & $\begin{array}{c}\kappa_{0} \\
\left.\mathrm{Nm}^{-1}\right)\end{array}$ \\
\hline Oxford & 5.2 & 1.9 & 10 & 0.01 & 50 & 4.4 & 0.85 \\
\hline Oxford & 5.2 & 1.9 & 30 & 0.02 & 30 & 12.3 & 0.8 \\
\hline Oxford & 5.2 & 3.4 & 10 & 0.01 & 51 & 3.5 & 1.2 \\
\hline Oxford & 5.2 & 3.4 & 30 & 0.01 & 40 & 5.9 & 0.7 \\
\hline P1 & 0.87 & 2.2 & 58 & 0.36 & 23 & 12 & 0.44 \\
\hline P1 & 0.87 & 2.2 & 31 & 1 & 37 & $(4.7)$ & $(0.33)$ \\
\hline$\overline{\mathrm{P} 2}$ & 0.28 & 3 & 9.5 & 2.4 & 47 & $(0.95)$ & - \\
\hline P2 & 1 & 5 & 9.5 & 1 & 37 & (1.5) & $(0.78)$ \\
\hline P2 & 1 & 5 & 9.5 & 2.5 & 49 & $(0.95)$ & $(0.5)$ \\
\hline $\mathrm{P} 2$ & 1 & 5 & 9.5 & 30 & 99 & - & - \\
\hline $\mathrm{P} 2$ & 1 & 5 & 9.5 & 100 & 150 & - & - \\
\hline P2 & 1 & 5 & 5 & 3.5 & 71 & $(0.4)$ & $(0.4)$ \\
\hline
\end{tabular}

is further evidence for the predicted intensity dependence of the boundary between the two regimes mentioned previously. The results in Table 4.1 with the smallest $N$ are those which give the most reliable lower limit for $\kappa_{0}$. Therefore the conclusion is that $\kappa_{0}=(1 \pm 0.2) \times 10^{-19} \mathrm{Nm}^{-1}$ for the trap in Oxford, $\kappa_{0}>0.4 \times 10^{-19} \mathrm{Nm}^{-1}$ for trap P1, and $\kappa_{0}>0.8 \times 10^{-19} \mathrm{Nm}^{-1}$ for trap P2. The results are inconsistent with the lowest values indicated in Table 2.1. By employing the technique of monitoring the variation of trap volume with number the two main problems which can lead to underestimates of $\kappa_{0}$ were avoided, namely residual multiple scattering of photons and excursions of the trapped cloud into the non-linear region of the confining force.

As well as providing values for $\kappa$, the studies lead to the following deduction about multiple scattering. This is that, as the number of atoms in the trap increases, the cloud radius starts to increase well before the temperature does. This is shown clearly by Fig. 4.5, where multiple scattering increased the trap volume by a factor of more than three when there were $5 \times 10^{5}$ trapped atoms. The study of temperature in [17], on the other hand, implies a temperature increase of less than $10 \%$ with this number of atoms. There is also evidence for this assertion in the results for trap P2 at $\Omega=\Gamma$ in Table 4.1. In this case the density remained constant $\left(\right.$ at $\left.\sim 8 \times 10^{10} \mathrm{~cm}^{-3}\right)$ for numbers of trapped atoms between $10^{5}$ and $10^{7}$, implying that multiple scattering was limiting the density when there were as few as $N \sim 10^{5}$ atoms in the trap. The conclusion for the phase-space density is that multiple scattering limits the phase-space density first by limiting the density; the temperature increases only at higher numbers of trapped atoms. 


\subsection{Nonlinearity of the confining force}

This section describes a study of the two-component regime, whose onset was observed at high magnetic field gradients and reduced light shifts and with a reasonably large number of atoms, typically greater than $10^{7}$.

The obvious signature of the spatial breakdown of $\kappa$ is the two-component density profile described in Sec. 2.2.3. To observe this the trap was loaded under normal conditions and then the magnetic field gradient was transiently switched to a higher value, and simultaneously the intensity was reduced and/or the detuning was increased. Images of the cloud acquired were along the two orthogonal directions with the framegrabbers during the subsequent $100 \mathrm{~ms}$. A sample two-component profile is shown by the filled circles in Fig. 4.6(a). This profile was obtained from a single horizontal line of a CCD image, and has been fitted with the sum of two Gaussians of differing radii and peak heights. For comparison the distribution of the same atoms at a much lower field gradient is also shown (the open circles); this exhibits a square profile, characteristic of the multiple scattering regime. It is important to emphasize that the peak densities of the two Gaussians in the two-component regime could differ by a factor of up to 100, making the background Gaussian very difficult to detect, despite it often containing roughly the same number of atoms as in the narrower Gaussian. In fact to aid the initial search for two-component profiles the two-dimensional CCD images were summed horizontally (or vertically), in preference to working with a single line profile only. This extra stage of integration exaggerated the height of the background profile with respect to that of the central component by roughly the ratio of the two radii of the Gaussians, and also served to improve the signal-to-noise ratio. It should also be appreciated that the background profile could be easily missed unless a sufficiently large area of the CCD image $\left(\gtrsim 1 \mathrm{~mm}^{2}\right)$ was viewed. This is clear from Fig. $4.6(\mathrm{a})$, which was captured with a $128 \times 128$ pixel grid (the largest grid size for which acquisition of sequential video fields was possible).

With the signal from the background Gaussian so weak (it was sometimes as low as the stray noise levels), it was important to be sure of the interpretation of a breakdown in confinement. As a first step it was confirmed that the shape of the profile was independent of the size of the imaging aperture, and thus not the result of spherical lens aberration (this would cause off-axis rays to spread out in the image plane, thereby inducing a trap profile possibly similar to the two-component profile observed). As a second test the profile was observed in momentum space. Fig 4.6(b) shows a TOF signal obtained under the same conditions as for Fig 4.6(a). The shape is clearly also the superposition of two Gaussians. The width of the background Gaussian corresponds to a temperature much greater than the Doppler limit, and therefore this is experimental evidence that only those atoms confined in the central region of the trap are efficiently cooled by the sub-Doppler mechanisms, as one would expect. Moreover, the number of atoms in the colder of the two velocity distributions was found to vary with the trapping parameters in the same way as the number in the narrower of the two density distributions (see below). For a third observation the decay rate of the number of atoms in each Gaussian was measured. For parameter values near the transition between 
Fluorescence profile (arb. units)

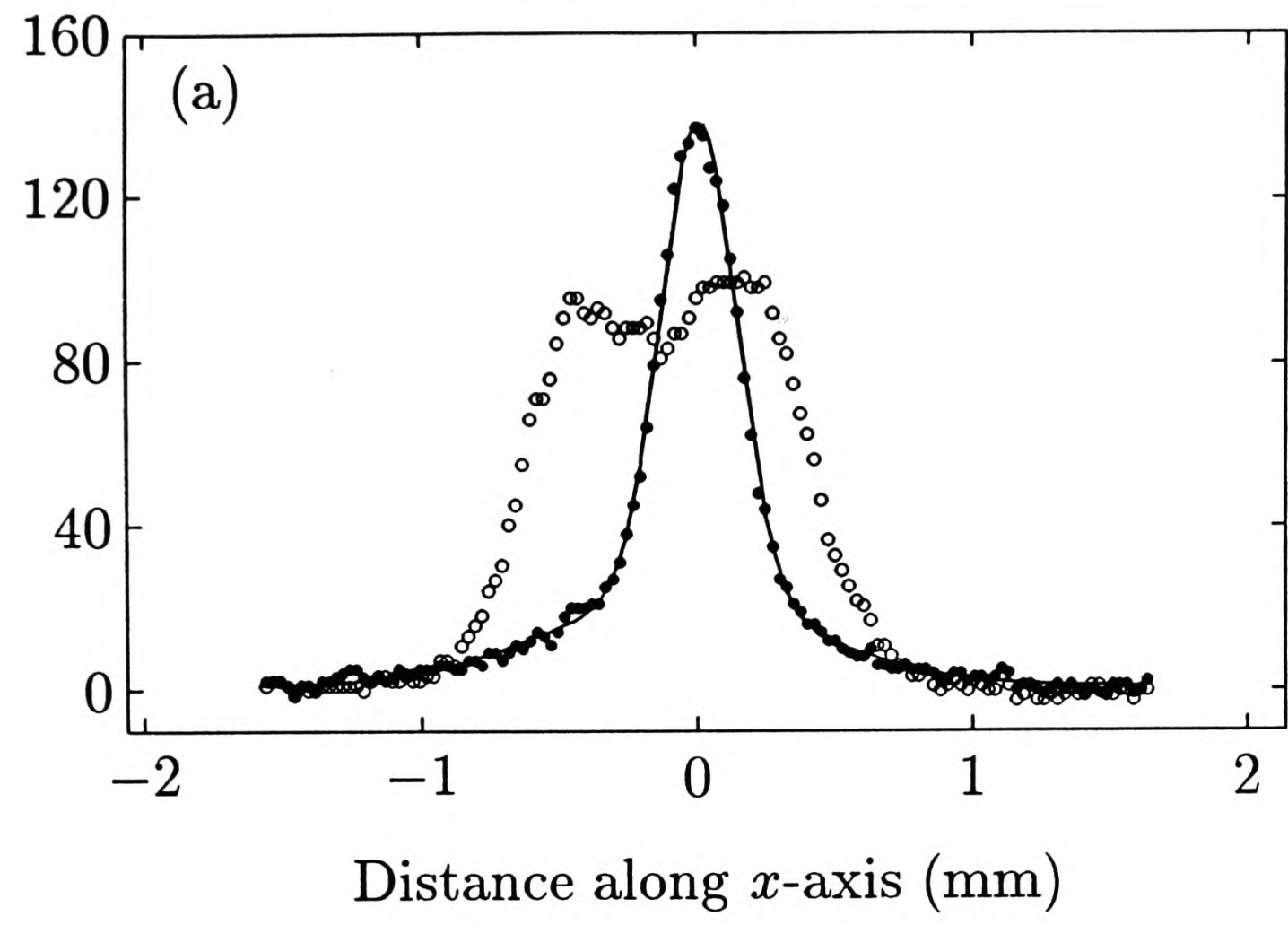

TOF signal (arb. units)

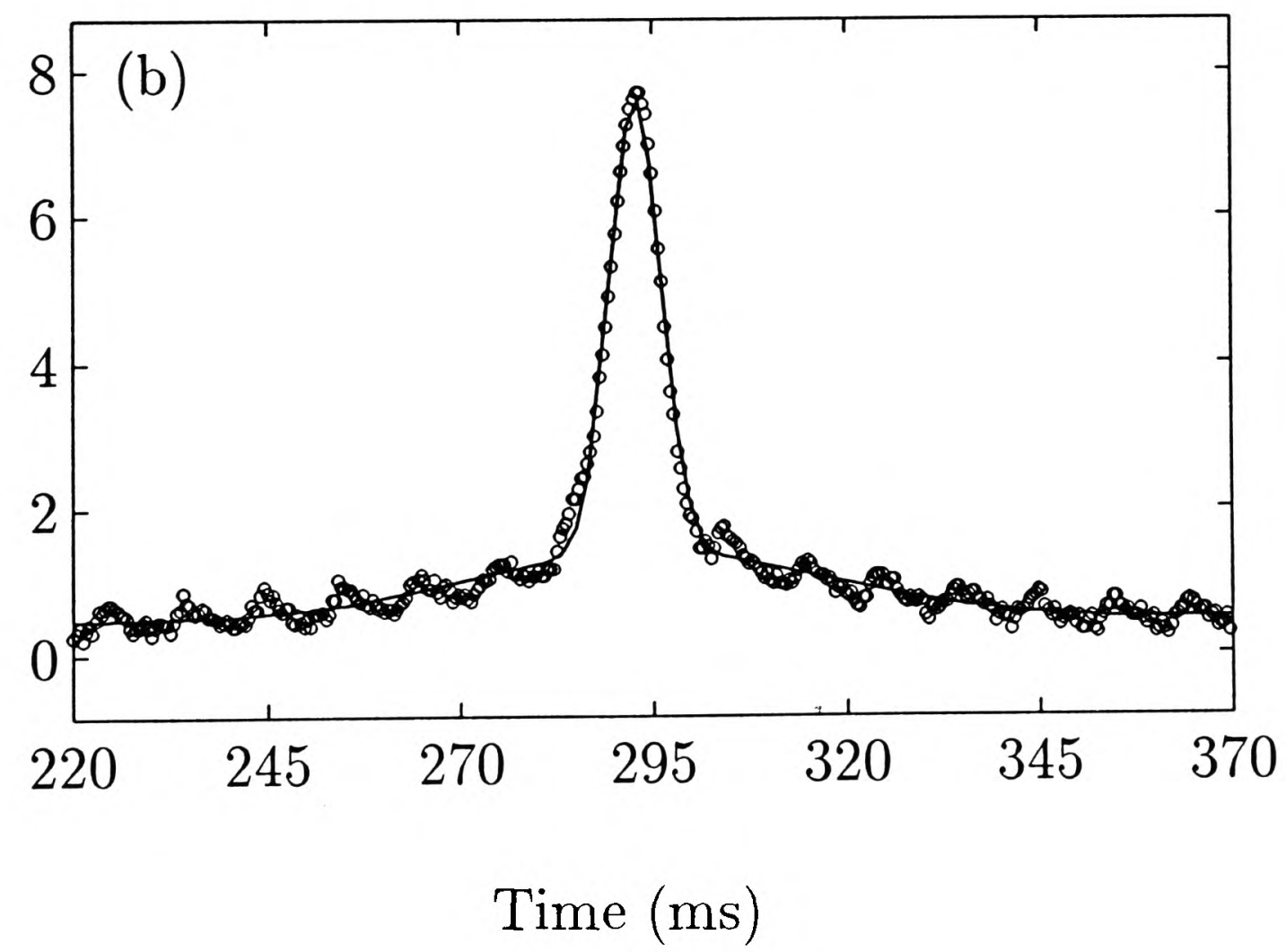

Figure 4.6: Observation of the two-component regime. (a) A horizontal line from the CCD image and (b) the corresponding TOF signal at $\Omega=0.75 \Gamma, \delta=5.7 \Gamma, N=2 \times 10^{7}$ atoms. In (a) the CCD image is shown for two values of the magnetic field gradient: $b=45 \mathrm{Gcm}^{-1}(\bullet)$ and $b=10 \mathrm{Gcm}^{-1}(\circ)$. The solid line through the latter is a fitted curve comprising the sum of two Gaussians. In (b) the TOF signal is shown only for the case $b=45 \mathrm{Gcm}^{-1}$; the fitted curve is also the sum of two Gaussians. The TOF signal at the lower field gradient (not shown) was well fitted by a single Gaussian. 
the two-component and multiple scattering regimes, the numbers in the central dense region and its diffuse surround were seen to decay at comparable rates, and it seems reasonable to attribute this to the weak dependence of the collision rate with the background vapour with escape velocity (from Eq. (2.37) $\tau \propto v_{e s c}^{1 / 3}$ at low scattering rates). However, well into the two-component regime the decay rate of the central component was observed to suddenly increase, and its lifetime typically fell below $200 \mathrm{~ms}$. Such a rapid change did not occur in the decay of the diffuse surrounding region. This transition may be interpreted as the point at which the trap is sufficiently weakened for hyperfine structure-changing collisions to be able to contribute to the trap loss. This additional decay mechanism is clearly more important for the central region because the density there is much larger. As final evidence for a spatial breakdown in confinement it was observed that the spatial shape of the background Gaussian is indeed sensitive to alignment of the trapping beams, as predicted in [77] and reported in [68], and as illustrated in Fig. 4.7.

Fluorescence profile (arb. units)

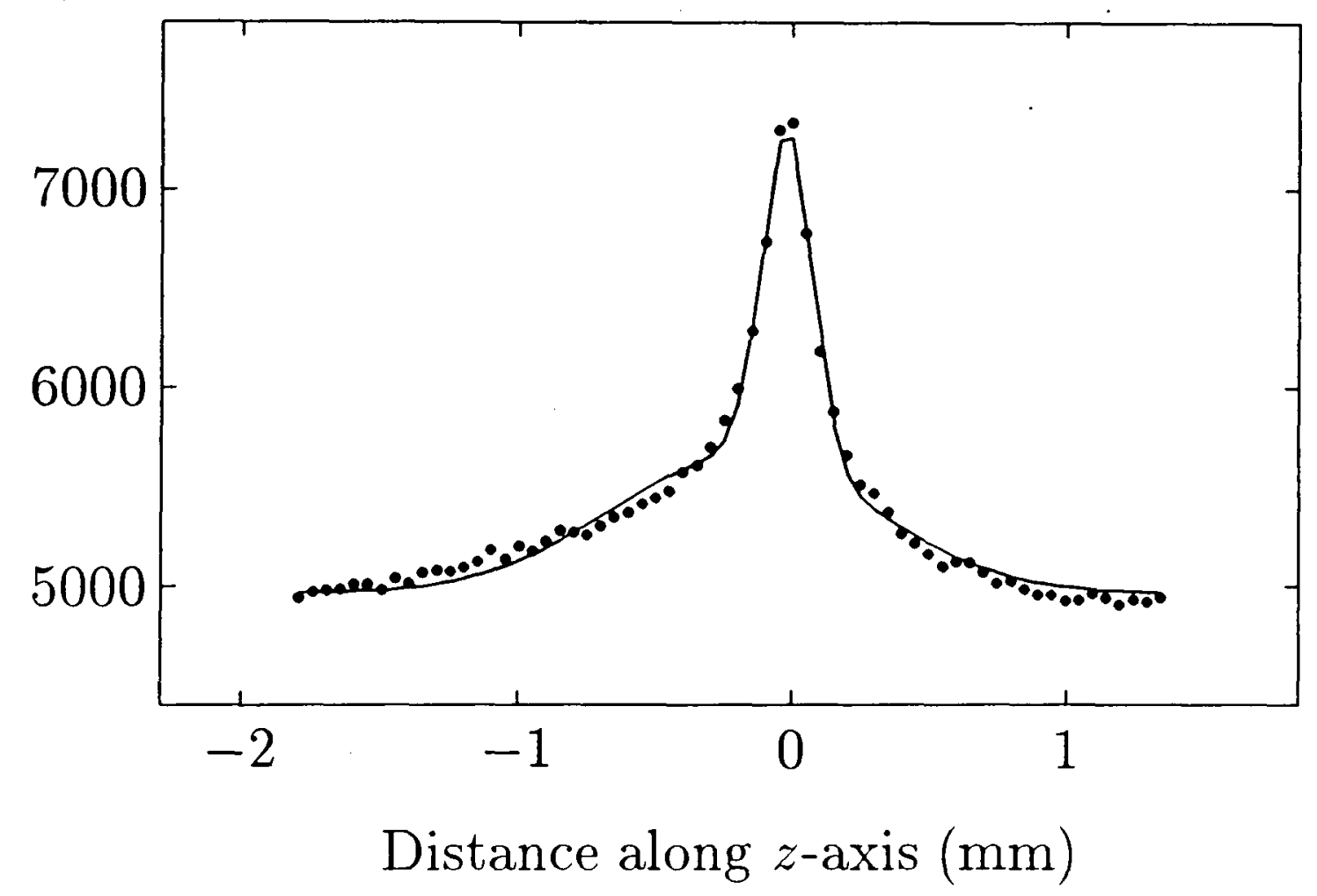

Figure 4.7: Sensitivity of the background Gaussian to alignment of the trapping beams. The filled circles represent the $z$-profile obtained by summing a CCD image in the $x$-direction, and the solid line the best fit sum of two Gaussians with the horizontal position of each floated. The parameter values were $\Omega=0.75 \Gamma, \delta=5.7 \Gamma, b=40 \mathrm{Gcm}^{-1}$, and $N=1.4 \times 10^{7}$ atoms. Notice how the process of summing has exaggerated the shape of the background Gaussian (compare with Fig. 4.6). The distribution of the weakly-confined atoms is clearly displaced with respect to those in the central region, and the displacement was observed to depend upon the alignment of the $z$-trapping beam.

Calculation of the number of atoms in the central component allows comparison of the observed behaviour with the predictions made in Secs. 2.2.3 and 2.2.4. The TOF signal was able to provide useful information about the variation of the relative 
number (the signal was not calibrated for absolute number) in the central region with different parameter values. This number was obtained as follows. The initial difference in the spatial profile of the two velocity distributions (on a distance scale $\sim 1 \mathrm{~mm}$ ) is negligibly small compared to the ballistic expansion of the cloud on falling through $42 \mathrm{~cm}$. Thus the two components each contribute a Gaussian to the TOF signal, which are similarly centred and with areas proportional to the number in that component. In fact to make meaningful comparisons one must additionally stipulate that the TOF probe beam illuminates all of atoms in the relevant component; a simple calculation for a $2.5 \mathrm{~cm}$ wide probe beam and a trap-probe separation of $42 \mathrm{~cm}$ indicates that due to the horizontal ballistic expansion of the cloud this is only the case for trap temperatures less than roughly $30 \mu \mathrm{K}$. Thus the TOF signal could be used to deduce number information about the central component only. A calculation of the number in the central component from the CCD image requires a little more thought because (a) in the spatial profile the "Doppler atoms" do not penetrate into the sub-Doppler region, and (b) in the process of summing the trap distribution along a particular direction (such as inevitably occurs when the fluorescence is imaged onto the CCD) signal is added from the diffuse surround onto that from the central region. Thus if the two-component profile is fitted with a sum of two Gaussians, the numbers calculated from each Gaussian individually do not correspond (exactly) to the populations of each component. One can proceed with an estimate of the correction as follows. The density distribution in the trap is well approximated by the sum of two Gaussians:

$$
n(\mathbf{r})=h_{1} e^{-r^{2} / \sigma^{2}}+h_{2} e^{-r^{2} / l^{2}}
$$

where $\sigma$ and $l$ are respectively the dimensions of the central peak and the diffuse background, and where for simplicity spherical symmetry has been assumed. We now suppose that rearranging the above expression into the following form can adequately represent the division of population between the central peak and the surrounding region:

$$
n(\mathbf{r})=\underbrace{\left(h_{1}+h_{2}\right) e^{-r^{2} / \sigma^{2}}}_{\text {central region }}+\underbrace{h_{2}\left(e^{-r^{2} / l^{2}}-e^{-r^{2} / \sigma^{2}}\right)}_{\text {Doppler region }}
$$

i.e. the diffuse surround only penetrates to roughly a distance $\sigma$ from the trap centre. It will be seen that the final division of population is insensitive to exactly how this fall-off occurs. Equation (4.7) implies

$$
\begin{aligned}
N_{\text {central }} & =\pi^{3 / 2}\left(h_{1}+h_{2}\right) \sigma^{3} \\
N_{\text {Doppler }} & =\pi^{3 / 2} h_{2}\left(l^{3}-\sigma^{3}\right) .
\end{aligned}
$$

Now if we deduce numbers $N_{1}$ and $N_{2}$ from the two Gaussians whose sum fits the CCD image integrated along a direction, then from Eq. (4.6)

$$
\begin{aligned}
& N_{1}=\pi^{3 / 2} h_{1} \sigma^{3} \\
& N_{2}=\pi^{3 / 2} h_{2} l^{3} .
\end{aligned}
$$


Thus

$$
\begin{aligned}
N_{\text {central }} & =N_{1}+\Delta N \\
N_{\text {Doppler }} & =N_{2}-\Delta N \\
\text { where } \Delta N & =N_{2} \frac{\sigma^{3}}{l^{3}}=N_{2} \frac{V_{1}}{V_{2}} .
\end{aligned}
$$

Typically $V_{2} / V_{1} \sim 100$ and $N_{2} / N_{1} \sim 2-5$ and thus it is seen that the error $\Delta N$ in using $N_{1}$ and $N_{2}$ to represent the two populations is in fact only a few percent. Thus clearly the exact distribution of the Doppler atoms in the central region is not important.

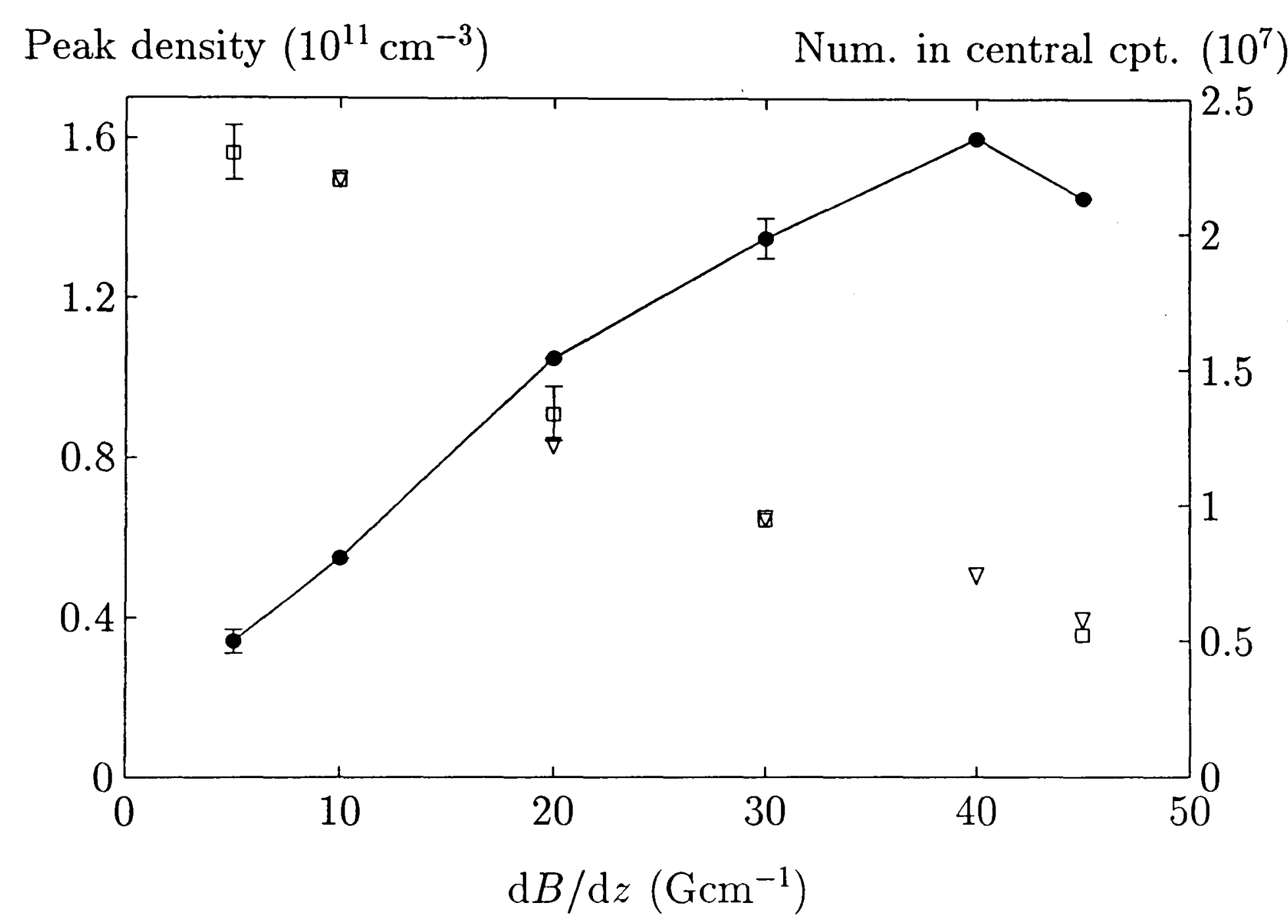

Figure 4.8: Density at the centre of the cloud $(\bullet)$, and number of atoms in the central component $(\square, \nabla)$, as a function of magnetic field gradient, in the two component regime. The laser parameters were $\Omega=0.75 \Gamma$ and $\delta=5.7 \Gamma$. The open squares show the number in the central component of the density distribution, as deduced from the CCD signal, while the triangles show the number in the cold component of the velocity distribution, as deduced from the time of flight signal. The fact that these two numbers agree is evidence that the colder atoms originate from the central part of the cloud.

Figure 4.8 shows the number of atoms in the central component calculated from both the density and velocity distributions, as a function of magnetic field gradient. The density of the central component is also shown (see Sec. 4.8 for a description of density measurements). The model of chapter 2 predicts a density proportional to ficld 
gradient if the atoms are in a regime limited by multiple scattering inside the sphere with radius $r_{l}$. This was only observed for field gradients up to about $b=30 \mathrm{Gcm}^{-1}$. At the higher magnetic field gradients the scaling law suggested by the model clearly no longer applies, for which we have no reasonable explanation. It seems unlikely from the measurements of the position damping time in Sec. 4.7 and of the cold collision rates in chapter 5 that this is due to a break-down of the static model at high field gradients (and thus high densities) caused by large collisional loss rates. The results imply that a useful increase in the density is only obtained for field gradients easily produced in the lab, less than $60 \mathrm{Gcm}^{-1}$. This was a conclusion of the work by Petrich et al. [68].

In Fig. 4.8 the number of atoms in the central peak exhibits the expected fall-off as the magnetic field gradient is increased. However, the decrease is roughly as $b^{-1}$ rather than the more rapid $b^{-2}$ predicted by Eq. (2.24). This may be interpreted as behaviour in the transition region between the multiple scattering and two component regimes. The observed radius of the central peak in this transition region varied roughly as $b^{-0.6}$, but for a MOT well into the two-component regime we should expect the radius to be roughly equal to that of the sub-Doppler region, $r_{l}$, with a dependence $b^{-1}$ [Eq. (2.19)].

A program of data acquisition and analysis was carried out for a range of different parameter values and is shown in Fig. 4.9(a). Complementary measurements for trap P1 are shown in Fig. 4.9(b). It was found that when the MOT was well into the two-component regime (i.e. not close to the solid line shown) the radii of the central components were indeed consistent with Eq. (2.19), though the restricted set of data points (see Fig. 4.9) is not large enough to confirm this equation unambiguously. At a field gradient of $40 \mathrm{Gcm}^{-1}$ in the Oxford trap, the radius of the central component was $84 \mu \mathrm{m}$, and $64 \mu \mathrm{m}$, for detunings of $8.6 \Gamma$, and $11.4 \Gamma$ respectively, and at $\Omega=0.75 \Gamma$. These figures are consistent with $r_{l} \propto \delta^{-1}$, and imply $C_{l}=1.4 \pm 0.3$. Closer to the boundary between regimes, the radius of the central component varied more slowly with $\delta$. In trap P1 with a field gradient $b=62 \mathrm{Gcm}^{-1}$, the radius of the central component was $45 \mu \mathrm{m}$ at $\delta=9.5 \Gamma, \Omega=0.9 \Gamma$; these figures imply $C_{l}=1.2 \pm 0.3$.

Some temperature information was also obtained. The temperature of the atoms in the central peak was observed to decrease with number. At fixed detuning the temperature above the molasses temperature decreased roughly as $N_{\text {central }}^{1 / 3}$ as the magnetic field gradient was increased, although there was not sufficient data to be sure of this. Because the change in density under these circumstances is roughly comparable to that of the number in the central component (see Fig. 4.8) the implication of this is that heating from multiple scattering is not strongly dependent upon density. This is not what one would expect as the optical thickness of the trap, which one may think of roughly as the probability that a scattered photon is reabsorbed before leaving the cloud, varies as $n \sigma r=n^{2 / 3} \sigma N^{1 / 3}$ in the multiple scattering regime. The temperature of the weakly-confined atoms was observed to vary in roughly the range $100 \mu \mathrm{K}-1 \mathrm{mK}$, i.e. always above the Doppler limit. On the other hand the variation of temperature with detuning and field gradient was similar to that exhibited by the strongly-confined atoms, i.e. not what one would expect for atoms cooled by purely Doppler forces. A possible reason for this is that the spatial transition between weak 

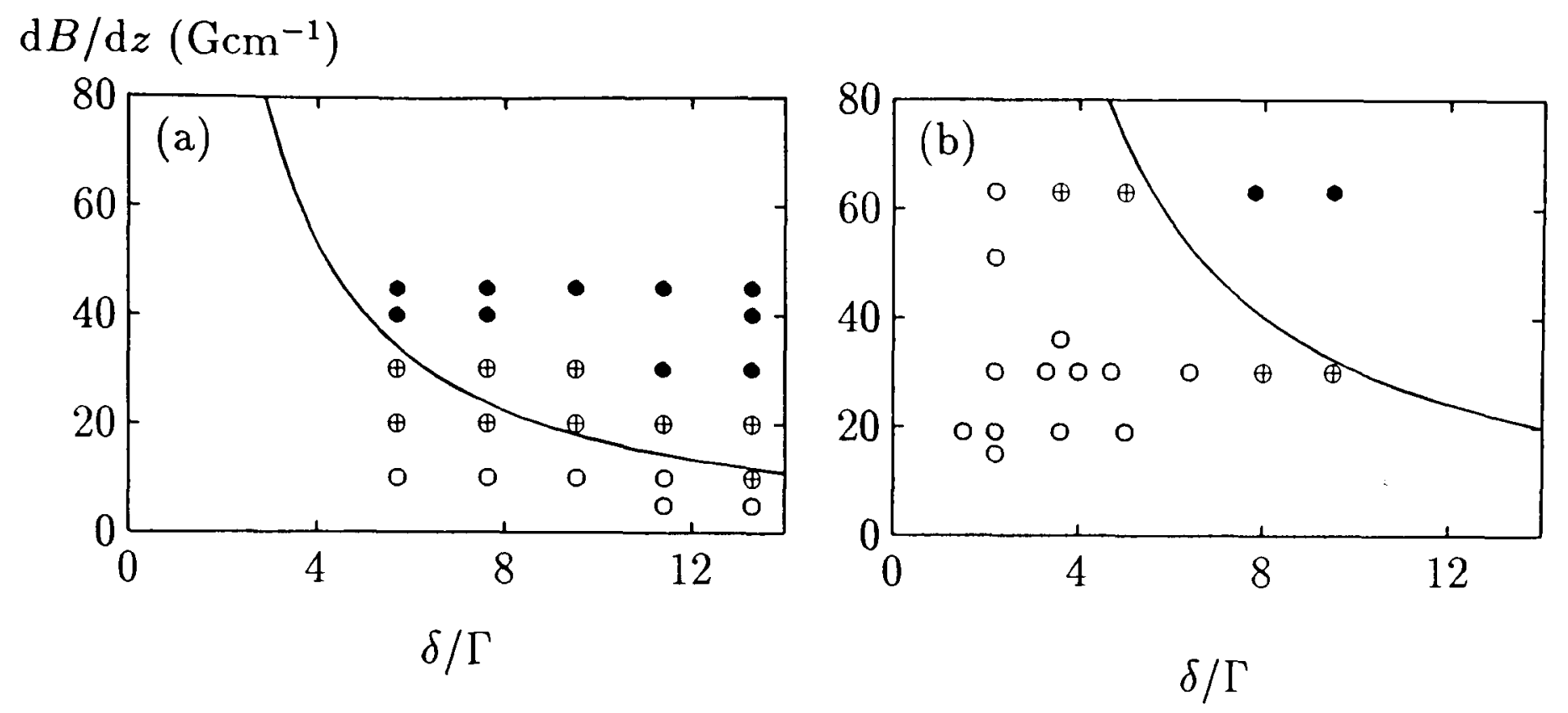

Figure 4.9: Parameter values at which density measurements were made. (a) Measurements on the Oxford trap, with $\Omega=0.75 \Gamma, N=2 \times 10^{7}$ atoms. (b) Measurements on trap P1, with $\Omega=0.9 \Gamma$, $N=5 \times 10^{6}$ atoms. The symbol shape indicates whether the observed density distribution consisted of a single narrow component (o), two components $(\bullet)$ (one narrow, one broad), or an intermediate distribution $(\oplus)$. The density distribution was also observed at other parameter values; the Figure indicates only those values for which a careful analysis was carried out. The solid curves in (a) and (b) show the position of the boundary predicted by the model with the empirical values $\kappa_{0} C_{\mathrm{MS}}=5 \times 10^{-18}$ $\mathrm{Nm}^{-1}$ (see Sec. 4.8), and $C_{l}=1.4$ for the Oxford trap, $C_{l}=1.2$ for trap P1.

and strong confinement is not predicted to be sharp (Fig. 2.1).

\subsection{The position damping time}

The technique of transiently switching the MOT parameters from those producing strong trapping forces and suitable for loading, to those giving much weaker confinement but which optimize sub-Doppler cooling and compression, was described in Sec. 2.3. The densities achieved by applying such a method are described in the Sec. 4.8. The procedure also enables a measurement of the position damping time for the following reason. Absorption by the background vapour and by the trapped cloud itself always gives an intensity imbalance between a trapping beam and its retroreflection. Consequently the trapped cloud is centred at a position displaced from the zero of magnetic field such that this difference in intensity is compensated by an imbalance in Zeeman tuning, i.e. so that there is no net absorption of trapping photons along any direction. Thus if the trapping parameters are changed so that the optical absorption is reduced or the magnetic field gradient increased, the cloud will move to a new equilibrium position nearer to the magnetic field zero. A measurement of the time to undergo the displacement then yields the position damping time $\tau_{p o s}(=\alpha / \kappa$ for spherical symmetry) for the new parameter values. This method contrasts to previous measurements of $\tau_{\text {pos }}[78,25]$ which have used either an external laser or uniform mag- 
netic field to displace the trap. The position damping time is an instructive quantity to measure because it provides a further check that the MOT damping forces are behaving as expected and, in combination with a measurement of the cold collision loss rate $\beta$, it allows one to estimate the collision-limited density [Eq. (2.32)].

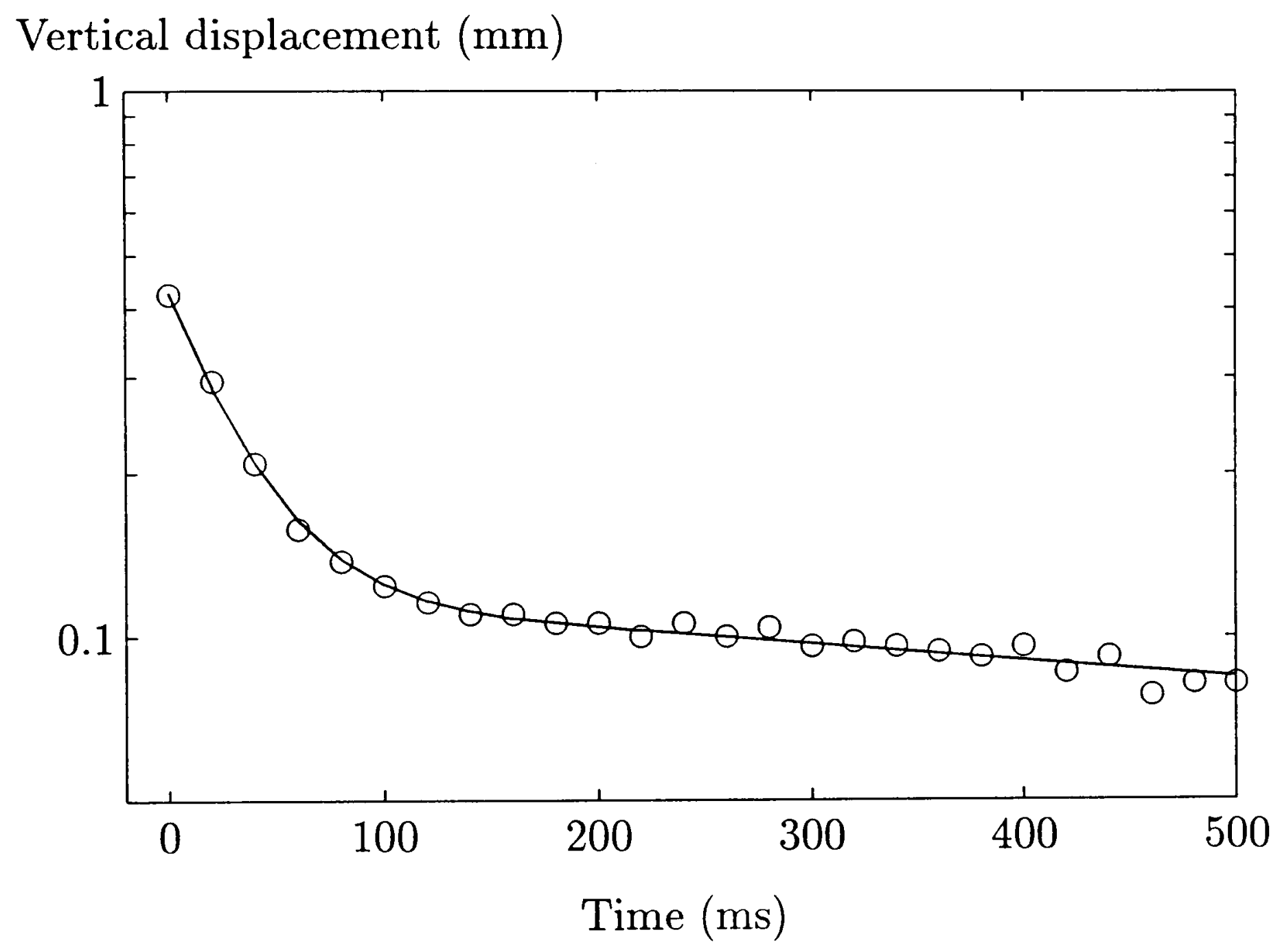

Figure 4.10: Displacement of the trap from the zero of magnetic field as a function of time; by weakening the trapping parameters $\{\delta, \Omega, b\}$ from $\left\{1.9 \Gamma, 1.4 \Gamma, 10 \mathrm{Gcm}^{-1}\right\}$ to $\left\{5.75 \Gamma, 0.75 \Gamma, 5 \mathrm{Gcm}^{-1}\right\}$ the trapping intensity imbalance was reduced and the MOT subjected to an impulse. The initial rapid change in position is a measure of the damping time $\tau_{\text {pos }}$ whilst the slow decay at large times arises from the decay of the trap number due to the weakened trapping.

The following procedure was adopted. The trap was fully loaded and then the parameters were switched to their desired values. About $50 \mathrm{~ms}$ before the switch the frame-grabbers for the two orthogonally-positioned cameras were triggered, and images of the trap were then acquired for several seconds. As mentioned in chapter 2 a reduced scattering rate nearly always results in a Gaussian-shaped trap; the profiles were thus fitted to such a function to find the displacement of the trap centre. Analysis was made of the vertical displacement of the images only because this corresponded directly to the vertical trapping direction $(z)$, and the use of two cameras provided a check for consistency. Alternate video fields are displaced vertically by one pixel (interlacing) and it was necessary to take this into account to extract a smooth variation of position 
with time, an example of which is shown in Fig. 4.10. The effect of position damping is clearly observed as the initial rapid change in displacement. However, a much slower change in position is also observed and this can be attributed to the decreasing optical depth of the trap as the trapped number decreases. To extract the damping time the variations were fitted with the superposition of an exponential and linear decay.

Figure 4.11 shows the variation of damping time with field gradient and detuning. The accuracy with which $\tau_{\text {pos }}$ is determined by the above method is limited by two factors. First, each video field gives a signal integrated over a period of $20 \mathrm{~ms}$, which imposes a limit to the lowest value of $\tau_{\text {pos }}$ that can be measured. This is not quite as bad as one might at first suppose. The integral of an exponential returns the same exponential, and thus one merely requires that enough trap movement is recorded to be able to establish a reasonable value for the decay time; to do this it is sufficient to record the trap position at roughly decay time spacings, i.e. it is possible to measure a damping time of about $20 \mathrm{~ms}$ with only $20 \mathrm{~ms}$ time resolution. The second limit to the accuracy arises from uncertainty in the floated value of $\tau_{\text {pos }}$ due to the additional decay in the trap position due to the decreasing trap number, and is estimated to be at around the $15 \%$ level.

One first notes from Fig. 4.11 that the magnitudes of $\tau_{\text {pos }}$ are indeed as have been measured previously [78, 25]. In Fig. 4.11(a) $\tau_{\text {pos }}$ varies with field gradient roughly according to the inverse relationship prediced in Sec. 2.2.2. It was not possible to resolve the decay for gradients larger than $10 \mathrm{Gcm}^{-1}$, confirming that at higher gradients $\tau_{\text {pos }} \lesssim$ $20 \mathrm{~ms}$. This is an important deduction for assessing the significance of collision-limited densities and will be further discussed in Sec. 4.8. Fig. 4.11(b) shows experimental evidence for the sensitivity of $\tau_{\text {pos }}$ to trap setup. The values and dependence measured are not in good agreement with those reported by Drewsen et al. [25]. However, in that work it was observed that $\tau_{\text {pos }}$ was sensitive to the polarization of the trapping beams along the relevant direction. As mentioned in Sec. 2.2.1 both the friction parameter and the spring constant in the MOT are sensitive to the amount of Sisyphus cooling present, and thus the beam polarizations. For the same reason one might suppose that $\tau_{\text {pos }}$ also be sensitive to the trap alignment, as Fig. 4.11(b) suggests.

\subsection{Density measurements}

\subsubsection{Introduction}

This section summarizes the understanding obtained of the compression forces in the caesium MOT, and represents careful measurements extending over a period of many months. It was originally hoped that by studying the density accurately it would be possible to compare its behaviour closely with the predictions outlined in chapter 2 , in a similar manner possible with MOT properties such as the spring constant. This would allow identification of the dominant density-limiting mechanism with a view to its possible suppression. However, it became apparent as the technique for measuring the density was sufficiently refined, that the density in the MOT is sensitive to certain aspects of the trapping setup, such as trap alignment, making it difficult to compare 

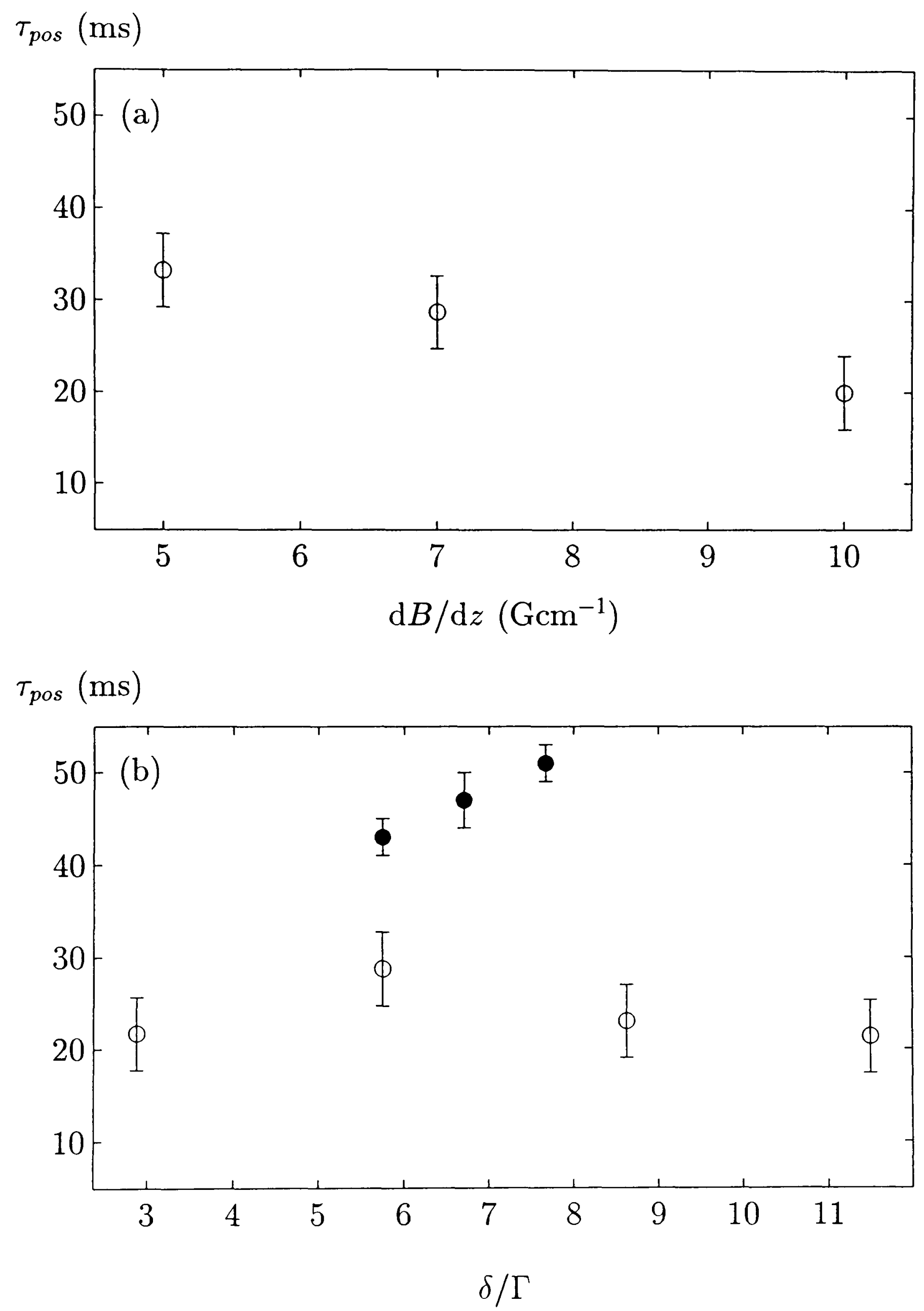

Figure 4.11: Measurements of the position damping time $\tau_{\text {pos }}(=\alpha / \kappa$ with spherical symmetry $)$ as a function of detuning and field gradient. For (a) the detuning was $\delta=5.75 \Gamma$ and for (b) the freld gradient was $b=7 \mathrm{Gcm}^{-1}$. The error bars represent an estimated uncertainty in determining $\tau_{\text {pos }}$ from the fits to the position relaxation. The open and filled circles represent measurements taken some six months apart and thus with differing trap alignments. The discrepancy in (b) between these two sets of data is therefore evidence for the sensitivity of $\tau_{\text {pos }}$ to the details of the polarization ficld. 
data sets quantitatively and to make definite assertions about the trends. This behaviour is perhaps a little disappointing in view of the seemingly robust nature of the MOT in some other respects. For example, it has been noted previously (such as in [25]) and was observed during our study that it is possible to trap atoms very easily with laser beams that do not have good quality incident wavefronts and whose polarizations are far from circular, i.e. it is not difficult to optimize the Doppler forces for loading. In addition, it was observed during the molasses temperature measurements (Sec. 4.4) that sub-Doppler temperatures are insensitive to the trap alignment and the distribution of intensity among the six beams, as previously remarked in [73] and [88].

However, it has been possible to reach qualitative conclusions about the density behaviour, aided by elimination of uncertainties in fundamental trap properties through the measurements described earlier in this chapter. The main deductions were as follows. Under nearly all circumstances the MOT density is limited by multiple scattering. Only with very few atoms does the trap pass into the temperature-limited regime (as was demonstrated in Sec. 4.5). From measurements of the position damping time and cold collision loss rates it has possible to deduce that none of the densities observed was close to being collision-limited. In the multiple scattering regime the Walker, Sesko, Wieman model only gives reasonable agreement with the observed behaviour at high scattering rates, where one expects the changes in density to amount only to small factors [Eq. (2.15)]. At lower scattering rates the density increases only slowly with decreasing light shift [roughly as Eq. (2.17) rather than Eq. (2.16)] and eventually at a low light shift $\left(\Omega^{2} / \delta \Gamma \sim 0.1-0.2\right)$ a breakdown in confinement is observed, i.e. the density begins to decrease. This requirement of having to be well into the multiple scattering regime for the expected theory to apply is an interesting observation because a similar conclusion was reached in the study of the dark MOT (chapter 6) from a different perspective. On the other hand the density does increase roughly linearly with increasing field gradient (for $b \lesssim 40 \mathrm{Gcm}^{-1}$ ), as expected in the multiple scattering regime. By transiently switching the parameter values from those used for loading to $b \sim 40 \mathrm{Gcm}^{-1}, \Omega^{2} / \delta \Gamma \sim 0.5$ the density was increased by a factor $\sim 15$ to $\sim 1.5 \times 10^{11} \mathrm{~cm}^{-3}$.

\subsubsection{The measuring technique}

The density was deduced by a fluorescence method; other possible approaches are discussed later. Two related techniques were investigated. The first was to convert into a total number of atoms the power emitted by the cloud into a known solid angle, and to deduce from this the density by measuring the cloud radius with a CCD: $n \propto N / r^{3}$. The power was calculated from either the signal from a photodiode circuit of known characteristics or by integrating the total signal falling on the CCD camera, calibrated for intensity. The other technique was to use the fact that, assuming the cloud is well imaged onto the CCD array, the video signal $S$ is proportional to the integral of the density along the "line of sight", so that the density can be deduced with the equation $n \sim S / r$.

For both methods one needs to know the scattering rate per atom for a given 
intensity and detuning, and for this the values $C_{1}^{2}=C_{2}^{2}=0.7$ measured in Sec. 4.3 were used. The second method has two potential advantages over the first. First, the radius only appears as a single power and thus the calculation is less sensitive to errors in measuring the trap dimensions. Secondly, one measures the density averaged along only one direction rather than three. With this reduced averaging one can make a better estimate of the peak density of a non-symmetric distribution; this can often be useful because at high scattering rates the effects of multiple scattering lead to decidedly non-Gaussian distributions. On the other hand, the method is more sensitive to imaging aberration, in particular to spherical aberration because this removes energy from the high intensity regions. These advantages and disadvantages were investigated directly by calculating the density with each method for identical CCD images. Even under loading conditions, when the trap profile was not usually Gaussian, the two approaches agreed to better than $\sim 30 \%$ in their estimates of the peak density, and at lower scattering rates the agreement was much better than this; these discrepancies are much less than other sources of error described below. It was decided that the first method should be the preferred approach for the reason that for low intensity images the signal-to-noise ratio is much improved by using information from the whole of the image, rather than from a single pixel signal $S$.

The specific steps in the calculation of a density were as follows. The CCD image from each camera (pointing along $x$ or $y$ ) was summed horizontally and vertically over the acquired grid to obtain "average" trap profiles with improved signal-to-noise ratio. Gaussian functions were least squares fitted to these four profiles to yield trap dimensions for the $x$ and $y$ directions, and two for the $z$ direction; the latter were compared to check for correct focussing of the two cameras. For each image the average background signal per pixel was estimated from the average of the offsets obtained from the two Gaussian fits; this background level was then used to calculate the total count due to trap fluorescence. For low intensity images it was sometimes necessary to sum only over a selected region of the acquired grid, i.e. to isolate as many background pixels from the summation as possible. This was satisfactorily achieved by selecting to sum only over a cross shape of chosen width, centred by eye on the trap. The pixel count was converted into a number of atoms and combined with the trap dimensions to give a density; in all that follows we refer to the peak density. For traps with a two-component profile the average (summed) profiles were fitted to the superposition of two Gaussians, as described in Sec. 4.6. However, in this instance the density of the central component was miscalculated by only $\sim 30 \%$ by fitting the profile just to a single Gaussian.

It is important to appreciate the uncertainties in the calculation of the density. There were several sources of systematic error. First, in determining the emitted power per unit solid angle the uncertainties in using either the photodiode (gain resistor tolerance, amps to watts conversion, collection solid angle, reflection losses) or the CCD (calibration, collection solid angle, reflection losses) were both estimated to be $\sim 15 \%$. Trap numbers deduced simultaneously with these two devices (with different collection optics) agreed to $\sim 20 \%$, thus confirming these estimates. Secondly, in converting the emitted power into a number of atoms one relies on the measurement $C_{1}^{2}=C_{2}^{2}=0.7$. 
At low scattering rates only the accuracy of the estimate of $C_{1}^{2}$ is significant; this was found to be accurate to $\sim 20 \%$ in Sec. 4.3. However, one should appreciate that $C_{1}^{2}$ might well vary with laser parameters or trap alignment, in which case this error will tend to be more random than systematic. Finally, the accuracy of the volume calibration was around $10 \%$. In addition there were various origins of random error. The most obvious of these was random fluctuation in both the trap fluorescence and the trap dimensions, the cause of which was not investigated. The time scale for the changes was observed to extend from much less than that of the $20 \mathrm{~ms}$ integration time of the CCD to somewhat longer, and densities calculated from consecutive video fields tended to differ in general by $\sim 10 \%$. A question to be addressed was the consequence of extracting the trap dimensions and number from a fit to a trap profile that was not quite Gaussian. One would expect the error incurred (in calculating the peak density) to vary with trapping characteristics such as number of atoms and the exact trap profile (i.e. the trapping beam alignment), and thus have most influence between different data sets. An estimate of the size of the effect was found by comparing the number totals calculated from the two "orthogonal" profiles for each CCD image, i.e. $N_{x} \propto A_{x} \sigma_{x}$ and $N_{y} \propto A_{y} \sigma_{y}$, where $A_{i}$ was the height of the summed profile in the $i^{\text {th }}$ direction with width $\sigma_{i}$. Typically $N_{x}$ and $N_{y}$ were observed to agree to better than $10 \%$. A third source of error was the small variation in selection of camera aperture and in correct focussing of the imaging optics. With the use of two cameras both of these could be checked for consistency and generally they contributed combined errors of less than $10 \%$. The principal reason for using two cameras was to account for the difference between the cloud dimensions in the horizontal plane (by symmetry one would suppose that the $x$ and $y$ dimensions ought to be equal). At high scattering rates there could be up to a factor of two difference in size, whilst at lower scattering rates agreement was generally good to $\sim 30 \%$. Finally, and most importantly, it was observed that the MOT density was sensitive to small changes in trapping beam alignment; this could be up to a factor of two for retroreflecting beams clearly misaligned $\left(>2 \times 10^{-3} \mathrm{rad}\right)$.

In light of the above discussion the following accuracy is suggested. The absolute value of the density was only certain to $\sim 50 \%$. After careful (and consistent) realignment of the trap, measurements with the same parameter values were generally comparable at the $30 \%$ level. Measurements within a given set of data could be compared to an accuracy of $\sim 10-20 \%$. These uncertainties are in general agreement with those quoted by Linquist et al. [56].

It is instructive to outline the method adopted for accurate alignment of the MOT, as this was important in attaining some degree of long term consistency in the data. The basic requirement was to ensure that the centres of the six trapping beams intersected at the magnetic field zero. The position of the latter was readily marked by formation of a steady-state trap, but only accurately enough with a high magnetic field gradient $\left(40 \mathrm{Gcm}^{-1}\right)$ to sufficiently reduce the displacement of the atoms from the field zero (caused by beam intensity imbalance). Indeed the capture efficiency of the MOT decreases rapidly with increasing field gradient [45] so that increasing the field gradient also ensured that the trapped number was small, reducing the optical depth of the trap and thus further the effect of beam imbalance. The procedure was then the following. 
Under such conditions the position of the field zero with respect to the centre of each of the independent trapping beams was assessed by moving a thin $(<1 \mathrm{~mm})$ object across the beam area until its shadow in the beam caused the trap to disappear. If necessary the positioning of the beam was then adjusted; in turn the retroreflection for that beam was also realigned (to an accuracy of better than $5 \times 10^{-4} \mathrm{rad}$, by eye). The procedure was then repeated for the other trapping directions. With these steps (sometimes with a process of iteration) it was possible to position the trap to within $1 \mathrm{~mm}$ of the magnetic field zero. Indeed more often than not the displacement was no more than one typically-sized trap diameter, i.e. of order a few hundred micrometres (See Fig. 4.10). Often with the alignment as described the profile of the fully-loaded trap $\left(10^{7}\right.$ atoms) at the high scattering rate used for loading was far from Gaussian, with the effects of multiple scattering causing instabilities and irregularities. To be able to obtain accurate density measurements it was always necessary to lower the scattering rate and reduce these effects.

Finally, other approaches to measuring the density should also be mentioned. The main disadvantage with the fluorescence technique described is the uncertainty in knowing the distribution of population among the ground state sublevels. One avoids this completely by optically pumping the atoms into a single sublevel and recording the fluorescence induced by a single beam, as was demonstrated in Sec. 4.3. However, this measurement is destructive and can be limited in detection sensitivity by the need for fast time resolution. There have been two other reported approaches to measuring densities but both suffer from essentially the same uncertainty as the fluorescence technique. The first is to measure the absorption of a weak probe tuned to a resonance (e.g. $F=4 \rightarrow 4^{\prime}$ in caesium) to give the absorption length of the cloud; the same probe can be scanned laterally across the cloud (or made wide enough to image the cloud onto a CCD) to find the trap dimensions, and thus deduce the density. Applications of this technique to the MOT have been reported by Wallace et al. [87] (who quote agreement with the fluorescence method to better than $20 \%$ ) and by Hoffman, Feng and Walker [41]. The second method is to deduce the number of atoms from the fluorescence induced by a TOF probe (e.g. see Drewsen et al. [25]). The uncertainty in the sublevel distribution in this instance is somewhat less than in the MOT because the electric field in one dimension is calculable (the TOF probe is normally retroreflected to prevent pushing of the atoms). Drewsen et al. [25] quote an agreement of this method of within a factor of two with the number deduced by fluorescence. We were not able to implement this method because the long time-of-flight time $(300 \mathrm{~ms})$ led to ballistic expansion outside the detection probe for atom temperatures greater than about $30 \mu \mathrm{K}$.

\subsubsection{The transient technique}

Typically the following sequence of events was employed. The trap was fully loaded with $\sim 10^{6}-10^{7}$ atoms using suitable parameters $\{\delta, \Omega, b\}$, such as $\left\{2 \Gamma, 2 \Gamma, 10 \mathrm{Gcm}^{-1}\right\}$. The frame-grabbing boards were then triggered, the parameters switched to their chosen values some $50 \mathrm{~ms}$ later, and the subsequent decay of the trap then monitored with 
the $x$ and $y$ cameras for up to a few seconds. Before each run it was important to measure the trapping beams intensities (the final intensity was calculated as a fraction of the initial value, and the initial value could wander on a timescale of minutes to hours depending on the particular stability of the alignment into the fibres), and to check for good injection-locking into the slave laser at the chosen detuning (changing the AOM frequency changed slightly the alignment of the locking beam - see Fig. 3.2).

The acquired frames from each camera were stored, analyzed and combined to extract the variations of number, volume and thus density as functions of time. The difference in trap fluorescence before and after switching was generally much larger than that covered by the 8-bit dynamic range of a standard CCD. Thus it was necessary to deduce the number of atoms from the emitted fluorescence at the final laser parameters; this was judged to be perfectly acceptable because over the range of final scattering rates the initial number deduced was constant at the level of the random fluctuations described above. A single camera was capable of detecting a change in trap number of up to a factor of roughly 50; if required this could be extended up to a factor $\sim 10^{3}$ by choosing complementary aperture settings for the two cameras.

To be able to interpret unambiguously the transient behaviour of the number and density as the MOT unloaded it was important to be sure that the final laser parameters -were sufficiently constant during the period of frame acquisition; this was particularly important for the approach to determining cold collision loss rates that is described in chapter 5. After some initial experiments in which the MOT detuning was changed by jumping the voltage supplied to the piezo-grating in the extended laser cavity, AOMs were employed to fix the final detuning exactly, as described in chapter 3 . This modification resulted in much improved TOF data [16]. Thermal drift of the trapping intensity after switching (resulting from the combined use of AOM and fibres) was corrected by the use of the feedback loop described in chapter 3. Generally drifts in the total intensity were less than $5 \%$ over a period of $5 \mathrm{~s}$.

An example of a decaying trap number and the corresponding density variation with time measured with the above procedure is shown in Fig. 4.12. Notice that because the switching of parameters was not synchronized with the triggering of acquisition to better than one field duration, the first density point is some combination of the initial and compressed density. Notice also that the density varies much more slowly than the number, as one would expect in the multiple scattering regime, but that it does not remain exactly constant. This was invariably observed to be the case and is the reason for the slightly non-exponential trap decay, as discussed in chapter 5.

Figure 4.13 demonstrates the necessity to measure the density transiently by showing a variation of the steady-state number and density as functions of increasing detuning. The point illustrated is that at only twice the detuning that one would use to load the trap $(\delta=4 \Gamma)$ the capture efficiency of the trap is much reduced. This results in loss of signal, but more significantly the MOT nears the temperature-limited regime for which the density decreases with number [Eq. (2.6)], as shown. Thus to investigate any reasonable range of detuning in the multiple scattering regime, the detuning must be changed transiently after first loading a large number of atoms. The capture efficiency of the MOT also decreases with increased field gradient and reduced trapping 


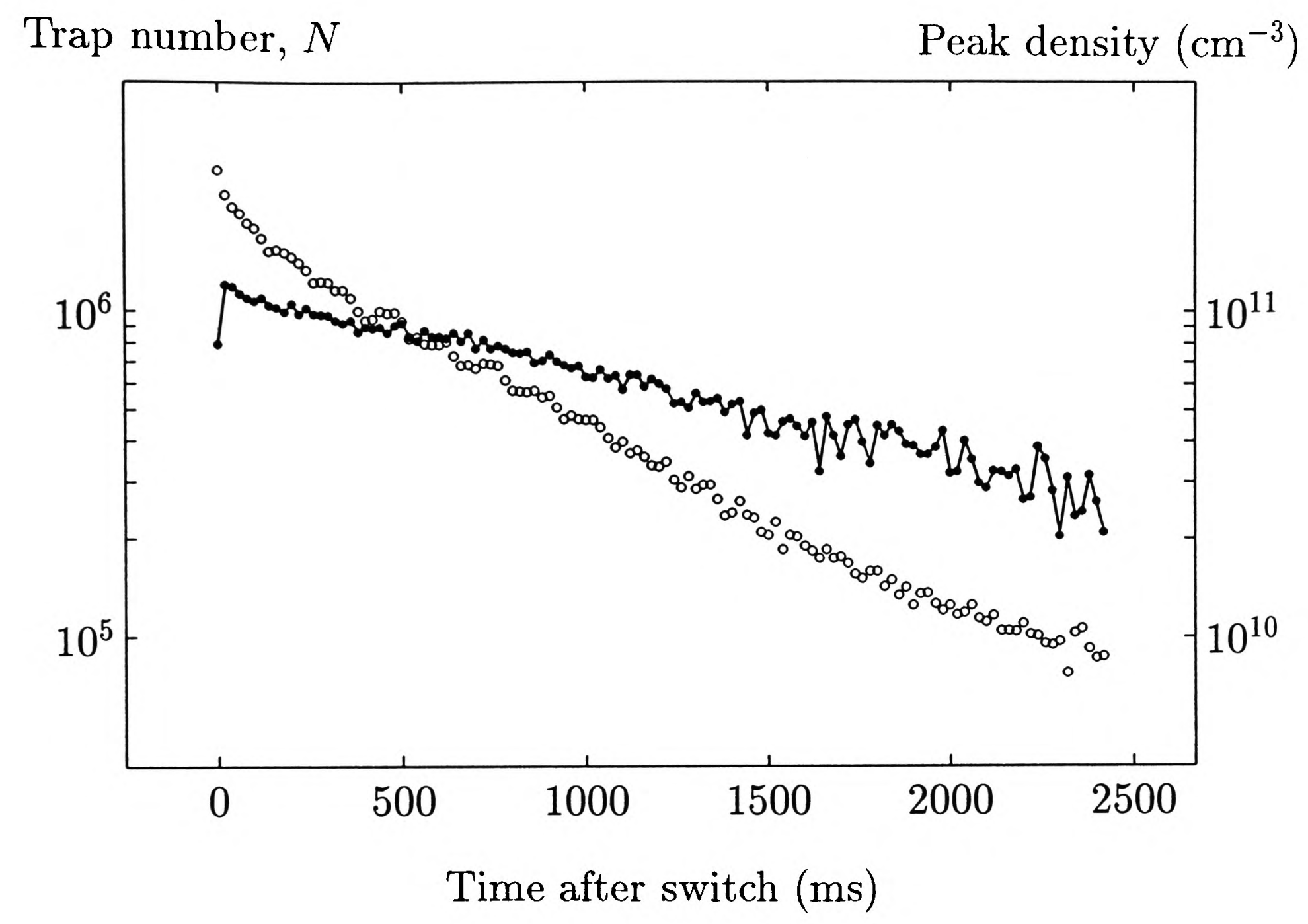

Figure 4.12: An example of the transient behaviour of the MOT number $(0)$ and peak density $(\bullet)$ after switching the parameter values $\{\delta, \Omega, b\}$ to $\left\{5.75 \Gamma, 0.95 \Gamma, 40 \mathrm{Gcm}^{-1}\right\}$ to increase the density. Note that the density compresses from its value under loading conditions (a few $10^{10} \mathrm{~cm}^{-3}$ ) to $\sim 10^{11} \mathrm{~cm}^{-3}$ in less than the $20 \mathrm{~ms}$ integration time of one video field - this is consistent with the position damping time measurements in Sec. 4.7 .

intensity, and thus the same conclusion also applies to varying these parameters.

\subsubsection{The density behaviour}

The features deduced of the density behaviour were the following. First, in a vapour cell MOT one is nearly always concerned with a density limited by multiple scattering; only if one takes pains to trap fewer than $\sim 10^{4}$ atoms can one observe a volume that is truly temperature-limited (Sec. 4.5). The signature of the multiple scattering regime is a trap volume that is not constant as the trap unloads or, equivalently, a density that decreases at a slower rate than the number, as shown in Fig. 4.12. The fact that the density does not remain exactly constant as the number varies may be interpreted as some degree of influence from the temperature-limited regime, i.e. some remnant of transitionary behaviour. In accordance with this suggestion the rate at which the density decreased was observed to be larger when the scattering rate was smaller or when there were fewer atoms. Typically with a total saturation parameter 

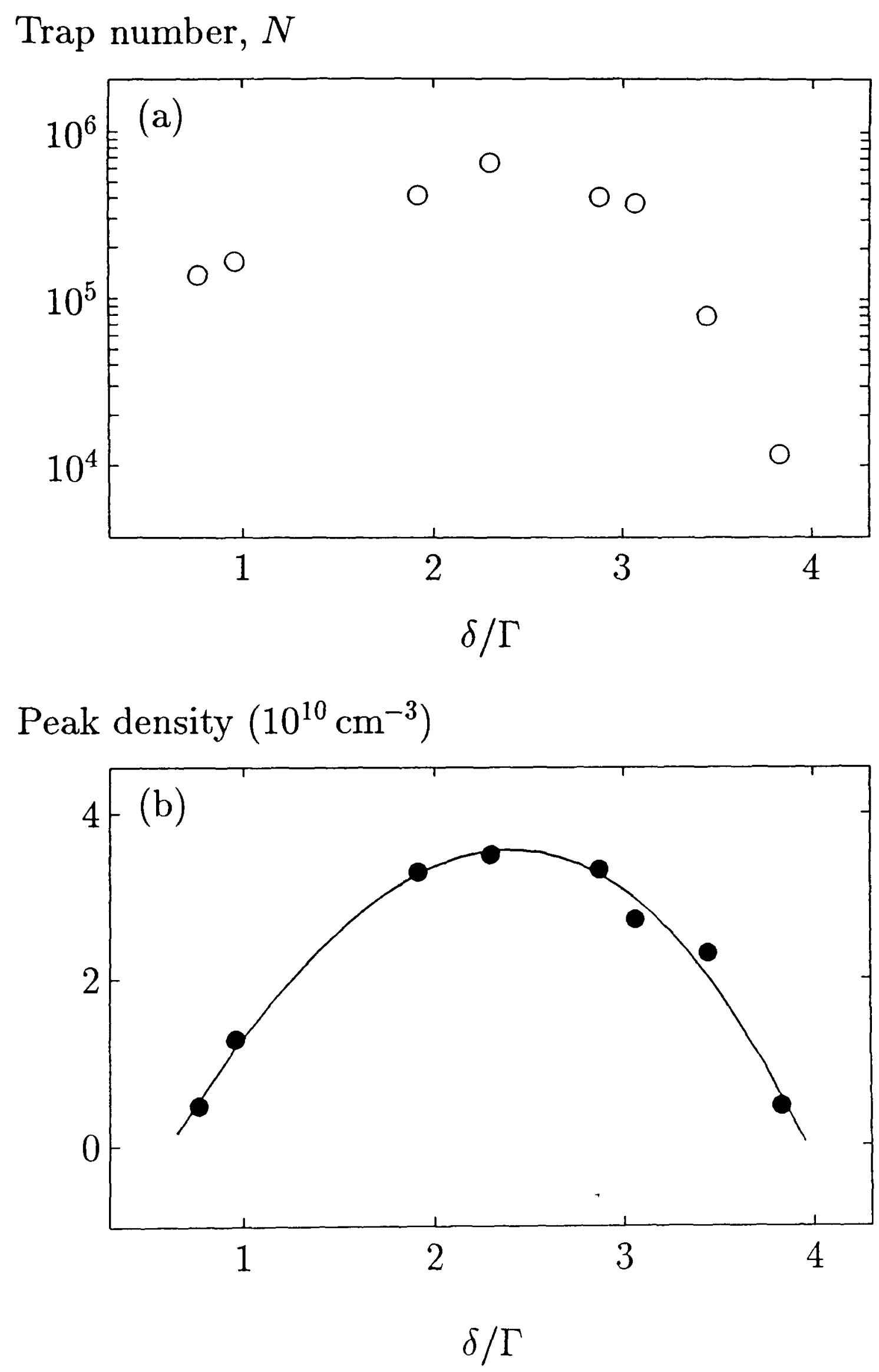

Figure 4.13: (a) Steady-state number and (b) the corresponding peak density as a function of increasing detuning, with parameters $\{\Omega, b\}=\left\{1.5 \Gamma, 15 \mathrm{Gcm}^{-1}\right\}$. The solid line in (b) serves only to guide the eye. The sharp fall-off in steady-state number with detuning illustrates the need for transient variation of detuning. 
$s_{\text {tot }} \sim 0.15$ and with $5 \times 10^{7}$ atoms, the number decayed at a rate approaching an order of magnitude larger than that of the density.

One observes that one cannot increase substantially a density limited by multiple scattering by reducing the scattering rate, contrary to the suggestion by Eq. (2.16). This same conclusion was deduced from measurements on traps $\mathrm{P} 1$ and P2, and has been hinted at previously [25, 68]. More specifically, Fig. 4.14 shows that the Walker, Sesko, Wieman model was found to hold reasonably well at high scattering rates, where from Eq. (2.15) one would expect only a small increase in density by reducing the saturation parameter. At lower scattering rates the density was observed to increase only slowly with decreasing light shift, roughly in accordance with Eq. (2.17). Eventually even this law was observed to fail, and for light shift parameters $\Omega^{2} / \delta \Gamma \lesssim 0.1-0.2$ a breakdown in confinement occurred, i.e. the density actually decreased, as shown in Figs. 4.15(a) and 4.15(b).

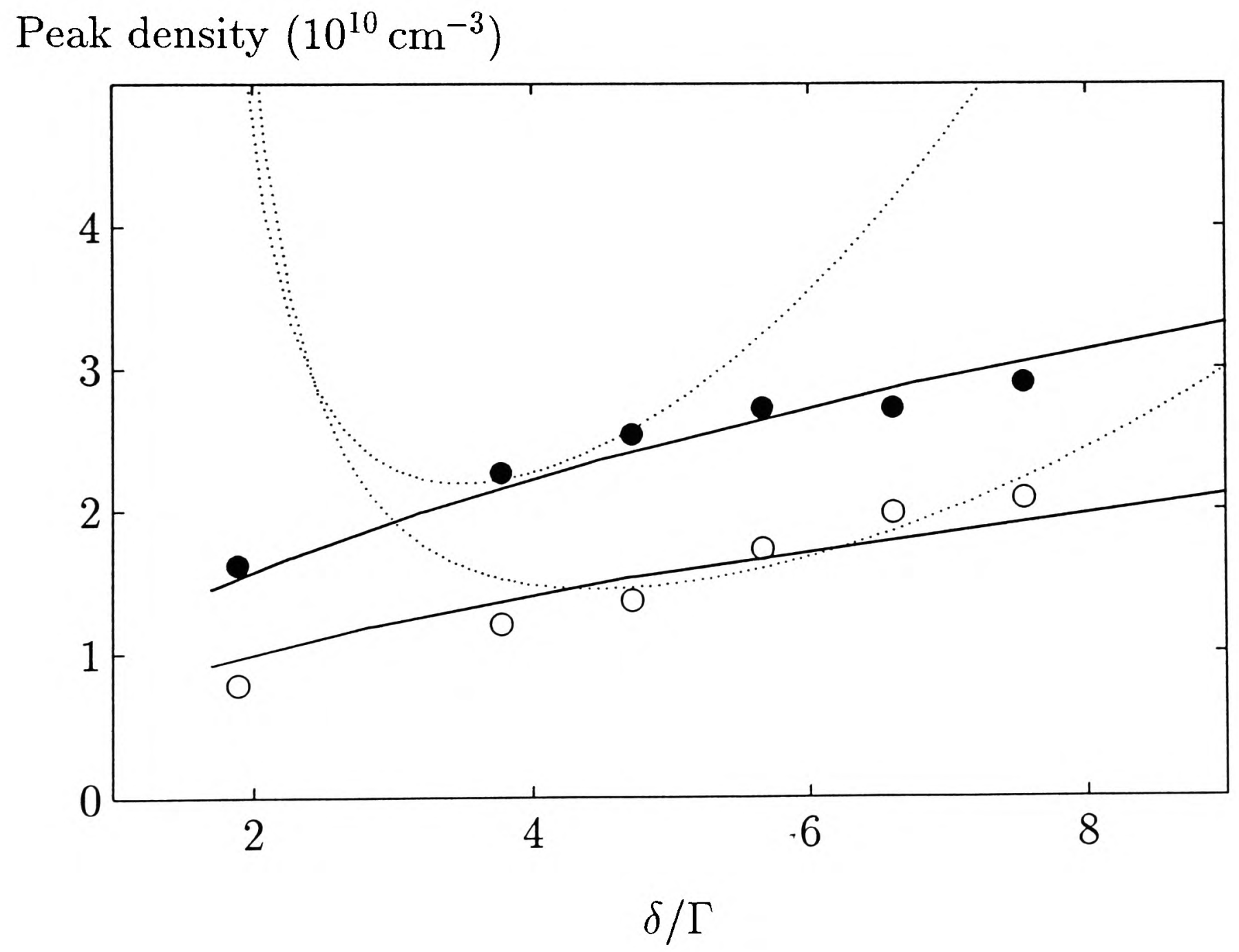

Figure 4.14: Failure of the Walker, Sesko, Wieman model. The circles represent transient peak densities as a function of detuning obtained for $\sim 5 \times 10^{7}$ atoms at $b=7 \mathrm{Gcm}^{-1}$ and for $\Omega=2.1 \Gamma$ $(0)$ and $\Omega=1.5 \Gamma(\bullet)$. The dotted curves are representative of the Walker, Sesko, Wieman model and arise from the evaluation of Eq. $(2.15)$, with the same scaling factor $(\approx 0.5)$. One sees that the agreement with the data is poor for $\Omega^{2} / \delta \Gamma \lesssim 0.4$. The solid lines are the empirical law Eq. (2.17) with $C_{\mathrm{MS}} \approx 63$ for the higher intensity and $\tilde{C_{\mathrm{MS}}} \approx 69$ for the lower intensity. The two data sets were taken a month apart. 

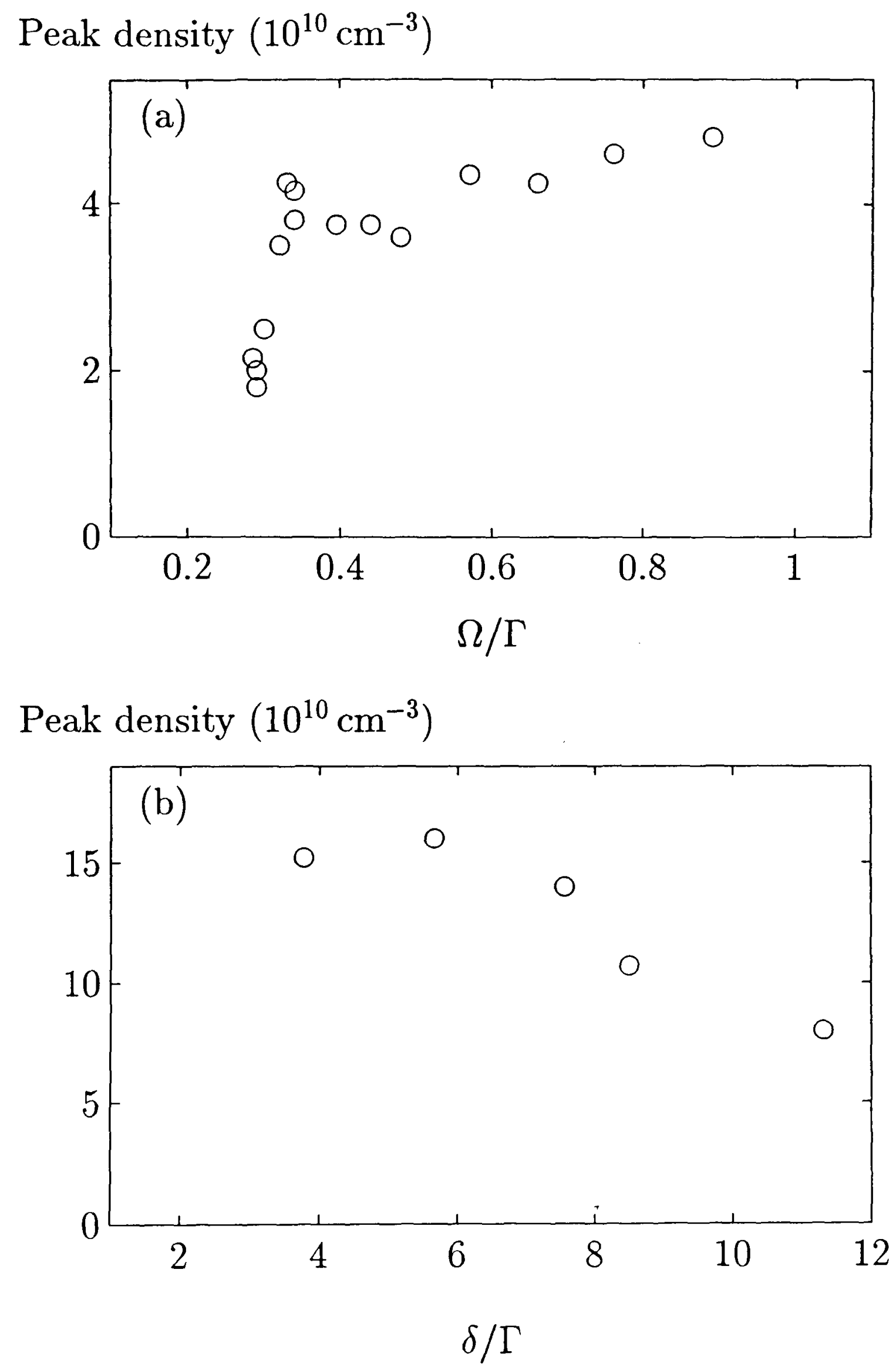

Figure 4.15: Evidence for spatial breakdown in confinement at low light shift. The density measurements in (a) were with $10^{6}$ atoms at $b=10 \mathrm{Gcm}^{-1}$ and $\delta=1.9 \Gamma$, and in (b) represent the density of atoms in the central component of a two-component profile at $b=40 \mathrm{Gcm}^{-1}$ and $\Omega=0.75 \Gamma$, with a number varying between $10^{6}$ and $10^{7}$ atoms depending on the detuning. In both situations the density has decreased measurably at a light shift parameter $\Omega^{2} / \delta \Gamma \sim 0.1$. 
The breakdown in confinement occurs at a reasonably low light shift, but nevertheless at a much larger light shift than that at which the sub-Doppler cooling mechanism was observed to fail (Fig. 4.3). The breakdown could be explained in terms of a reduction of the spring constant, and this would be in agreement with the findings of Wallace et al. [88] that were briefly discussed in Sec. 2.2.1. In that work evidence for such a reduction was presented, and indeed was observed to occur at $\Omega^{2} / \delta \Gamma \lesssim 0.2$, a factor $\sim 4$ larger than the shift at which the temperature started to increase. However, it is not possible to determine unambiguously from the results presented whether that reduction was due to the MOT entering the two-component regime and so experiencing a reduced average restoring force, or whether, as the results presented here suggest, the confinement process was vanishing altogether.

Although the multiple scattering-limited density was found to be relatively insensitive to the laser parameters, it could be substantially improved by increasing the field gradient, as already shown in Fig. 4.8. This was the only means to compress the MOT density to in excess of $10^{11} \mathrm{~cm}^{-3}$. Figure 4.8 shows that useful increases are only obtained for gradients up to about $60 \mathrm{Gcm}^{-1}$; using gradients higher than this simply decreases the extent of the strongly confining region at the trap centre, thus reducing the maximum number that one can confine to high density. With a combination of an increased field gradient $\left(40 \mathrm{Gcm}^{-1}\right)$ and a somewhat reduced light shift $\left(\Omega^{2} / \delta \Gamma \sim 0.3-0.5\right)$ maximum densities of $1-2 \times 10^{11} \mathrm{~cm}^{-3}$ were attained.

Comparing measurements from the three independent traps allowed an assessment of the sensitivity of the MOT density to optical setup. This is conveniently quantified by an estimate of $C_{\mathrm{MS}}$ for each case. For the Oxford trap numerous measurements, including those of Figs. 4.14 and 4.15, gave

$$
C_{\mathrm{MS}} \kappa_{0}=(5.5 \pm 2) \times 10^{-18} \mathrm{Nm}^{-1}
$$

where the error bar accounts for the average $30 \%$ discrepancy between data sets. Using $\kappa_{0}=(1 \pm 0.2) \times 10^{-19} \mathrm{Nm}^{-1}$ deduced from Sec. 4.5 gives $^{2} C_{\mathrm{MS}}=55 \pm 25$.

Measurements on trap P1 showed the density limited by multiple scattering to scale roughly as $b^{0.8} \delta^{0.4}$ for a majority of measurements. Imposing the law (2.17) gave a least squares fit $C_{\mathrm{MS}} \kappa_{0}=(5 \pm 1) \times 10^{-18} \mathrm{Nm}^{-1}$, which with $\kappa_{0}=(1 \pm 0.5) \times 10^{-19} \mathrm{Nm}^{-1}$ implies $C_{\mathrm{MS}}=50 \pm 25$.

Trap P2 yielded a value $C_{\mathrm{MS}} \kappa_{0}=(12 \pm 3) \times 10^{-18} \mathrm{Nm}^{-1}$ which is a factor of about two larger than those obtained in the other two traps. There is the possibility that this improved density is evidence for the sensitivity of the density to the optical quality of the trapping beams, as the main difference in the optical setup of trap P2 was that it was composed of six independent trapping beams, rather than three retroreflected beams. If this is the case then it implies that refraction and diffraction of the trapping beams by the MOT changes the retroreflected travelling wave sufficiently to halve the confining force.

Finally, it was possible to deduce that none of the densities observed were limited by collisions. It is clear from the equations presented in Sec. 2.3 that the transient behaviour of a density limited by collisions would be identical to one limited by multiple

\footnotetext{
${ }^{2}$ This value of $C_{\mathrm{MS}}$ was erroneously quoted in [83].
} 
scattering only, except that the maximum compression attained would be reduced. Thus unambiguous confirmation of the existence of a collision-limited density would be the observation of an improvement in density after $\beta$ had been reduced. Alternatively if the expression (2.32) for the collision-limited density is shown to be much larger than the densities measured then this is convincing evidence that collisions are not significant. This was indeed found to be the case, an example of which is the following. From Fig. 4.11 the position damping time at $\delta=5.75 \Gamma$ and $b=7 \mathrm{Gcm}^{-1}$ was measured to be about $30 \mathrm{~ms}$. The measurements of the cold collision loss rates in chapter 5 demonstrated that generally the largest values of $\beta$ in caesium arise from hyperfinechanging collisions, and are $\sim 10^{-10} \mathrm{~cm}^{3} \mathrm{~s}^{-1}$. Evaluation of $n_{\mathrm{CL}}$ with this value of $\beta$ gives the minimum possible collision-limited density, and is $n_{\mathrm{CL}}=3 / \tau_{\text {pos }} \beta \sim 10^{12} \mathrm{~cm}^{-3}$. By contrast the actual density measured was $\sim 5 \times 10^{10} \mathrm{~cm}^{-3}$, confirming the initial assertion. One should note that $\tau_{\text {pos }}$ scales with field gradient so that the densities in excess of $10^{11} \mathrm{~cm}^{-3}$ that were obtained at high values of $b$ were no nearer to being collision-limited that the example just given.

\subsubsection{Comparison with previous work}

A large number of publications have cited MOT densities of several times $10^{10}$ atoms per $\mathrm{cm}^{3}$, generally quoted for conditions typical for loading. There have not been many reported measurements on compressed traps. Measurements at high magnetic field gradients were reported in [68], with quoted compressions of order $2 \times 10^{11}$ atoms per $\mathrm{cm}^{3}$, in broad agreement with the results presented here. On the other hand, a careful set of measurements is reported in [25] which is not in good agreement with our results. In this work a MOT made from six independent beams was used and so is comparable to trap P2. At low laser intensities $\left(\Omega^{2}<0.5 \Gamma^{2}\right)$ the densities reported in [25] are between 4 and 6.5 times higher than those measured under comparable circumstances in trap P2, whilst at higher intensities $\left(\Omega^{2} \sim \Gamma^{2}\right)$ they are more than a factor 20 higher. It is not clear how to explain such a large discrepancy. The possibility of sensitivity to trapping beam quality has been mentioned, but it seems unlikely that this could produce such a large difference in confinement.

\subsection{Phase-space density}

From the prior discussions it is clear that a means to attain a high phase-space density is simply to increase the magnetic field gradient. This is demonstrated by Fig. 4.16 which shows measurement of $\rho$ in the central component of a two-component trap as a function of $b$. The increase of the spatial density was very similar to that shown in Fig. 4.8. In fact the increase in $\rho$ is also due to a reduction in temperature as the number in the central component falls off, as discussed in Sec. 4.6 and as shown in Fig. 4.16. From the discussion in Sec. 4.6 one would expect that at higher field gradients than those shown the density would remain roughly constant at $\sim 10^{11} \mathrm{~cm}^{-3}$, but that the temperature would continue to decrease. Thus one would hope that eventually a molasses temperature would be combined with a density $\sim 10^{11} \mathrm{~cm}^{-3}$, yielding, in this 


\section{Phase-space density, $\rho \quad$ Central component temperature $(\mu \mathrm{K})$}

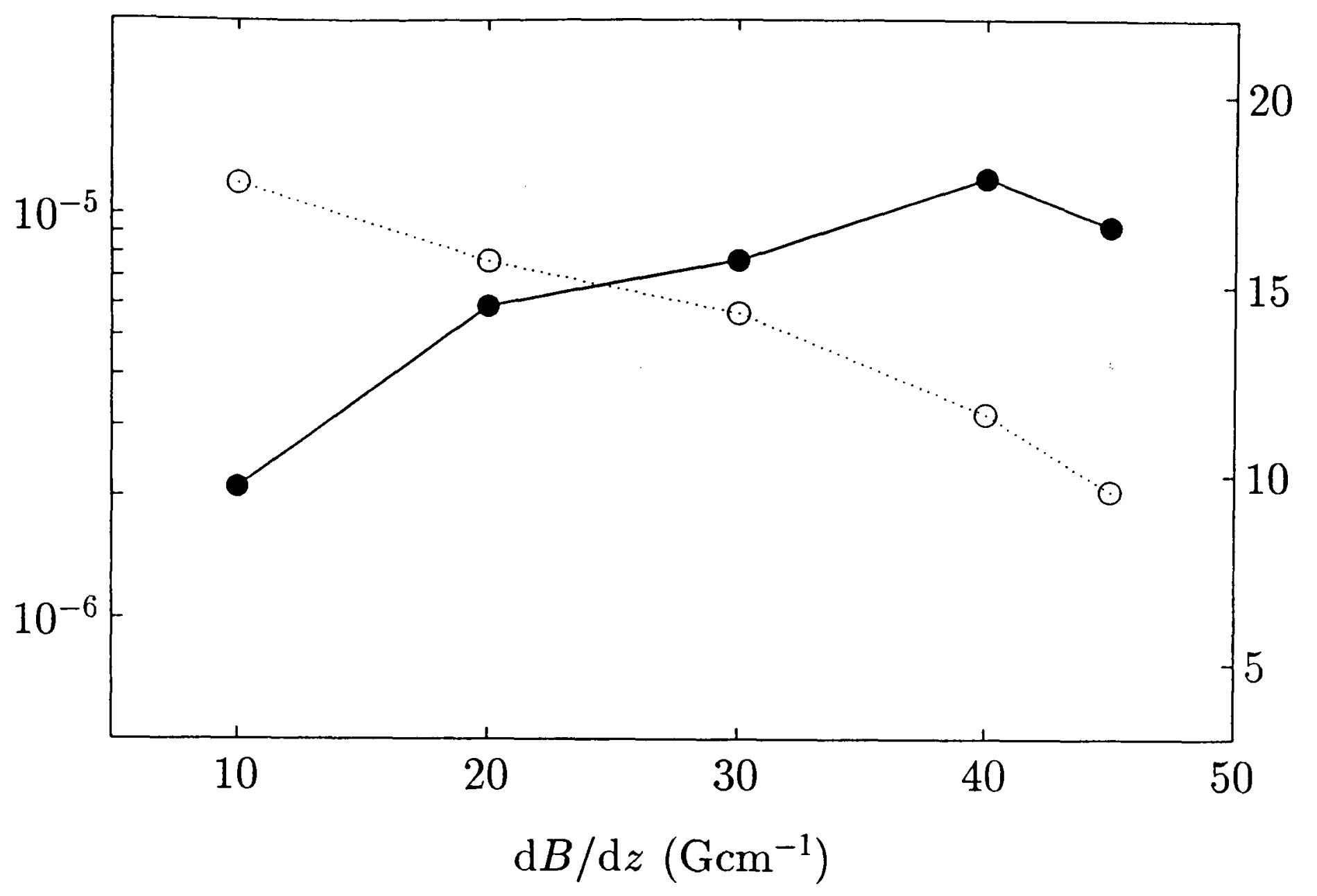

Figure 4.16: Phase-space density versus field gradient. At $\delta=7.6 \Gamma$ and $\Omega=0.75 \Gamma$ measurements of the temperature (O) and density (similar variation to that shown in Fig. 4.8) in the central component of the phase-space distribution were combined to give $\rho(\bullet)$ [Eq. (1.2)]. With increasing field gradient the number in the central component, and thus the excess heat from multiple scattering, is reduced; for these parameter values the molasses temperature from Fig. 4.3 is predicted to be about $5 \mu \mathrm{K}$.

particular instance, $\rho \sim 3 \times 10^{-5}$ (although with a small number of atoms, probably less than $10^{6}$ ). This is an increase in phase-space of some three orders of magnitude over that under typical loading conditions.

From the above discussion it might be hoped that it would be possible to combine the maximum MOT density of $\sim 2 \times 10^{11} \mathrm{~cm}^{-3}$ with the minimum molasses temperature of $\sim 3 \mu \mathrm{K}$ (Fig. 4.4) to give $\rho \sim 10^{-4}$. In fact due to the reduction in confinement at low light shift previously discussed this would be difficult to achieve in steady-state MOT. This is demonstrated quantitatively in Fig. 4.17 which shows the phase-space density resulting from a combination of density measurements (normalized to $30 \mathrm{Gcm}^{-1}$ ) and expected molasses temperatures. One sees that for light shift parameters below about $0.1, \rho$ falls below the $\left(\Omega^{2} / \delta\right)^{-2}$ prediction [Eqs. (2.9) and (2.17)]. Equivalently one may say that as far as optimizing the phase-space density is concerned cooling is effectively limited to $\sim 7 \mu \mathrm{K}$. It is thus suggested that the maximum value of $\rho$ that one can obtain in the MOT is about $5 \times 10^{-5}$.

A means to avoid the breakdown in confinement is to create the required conditions 


\section{Phase-space density, $\rho$}

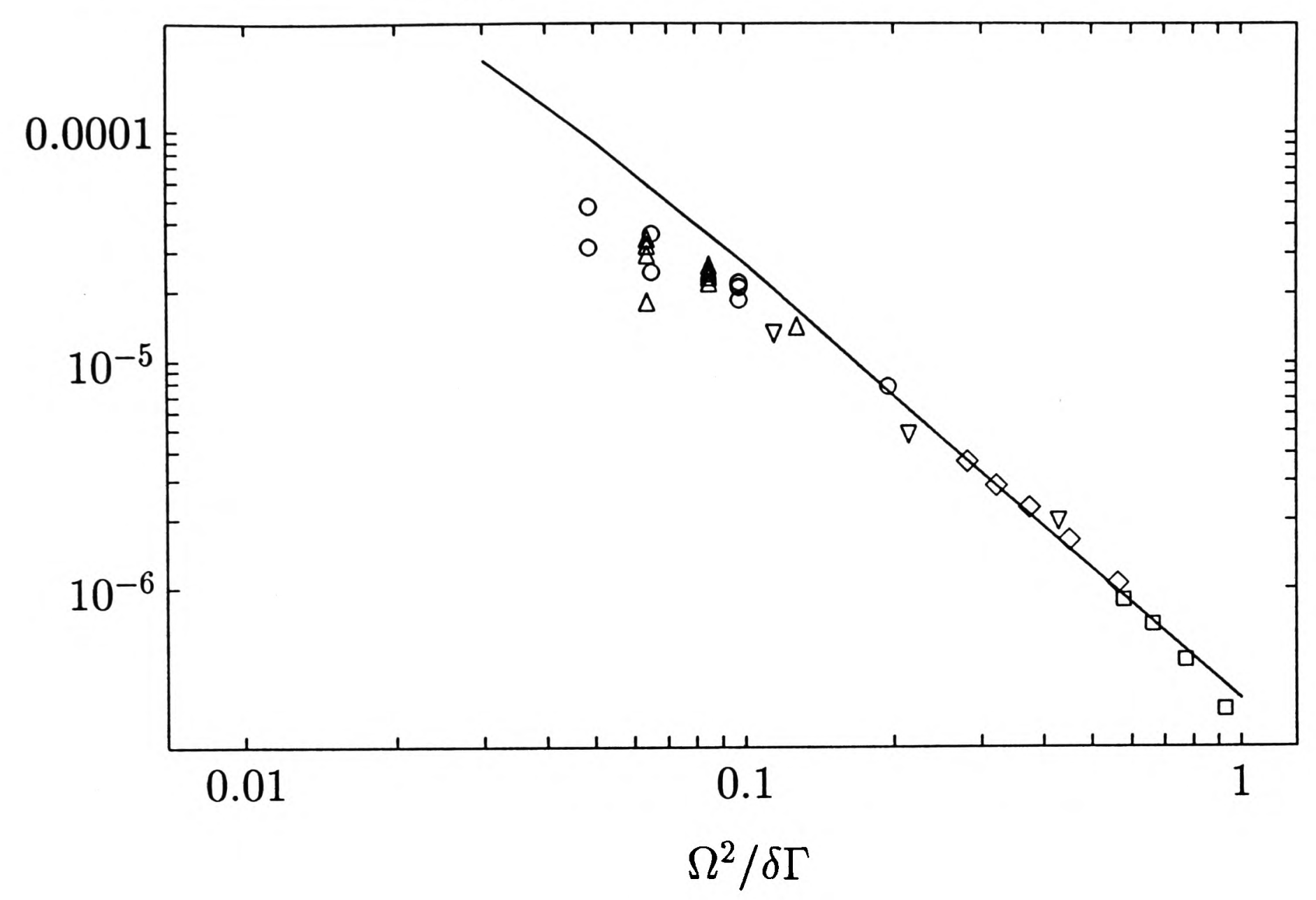

Figure 4.17: Phase-space density as a function of light shift, normalized to $b=30 \mathrm{Gcm}^{-1}$ with the use of Fig. 4.8. The spatial densities were measured and the temperatures taken to be those of molasses (Fig. 4.3). Each type of symbol corresponds to a different data run, with the accumulation of all data spanning a period of over six months. The values of $\Omega / \Gamma$ are as follows: $1.15(\nabla), 0.75(\Delta), 0.75(0), 1.5$ $(\diamond), 2.1(\square)$. The solid line is the prediction of the empirical model with $C_{\mathrm{MS}} \kappa_{0}=5.5 \times 10^{-18} \mathrm{Nm}^{-1}$.

for a high phase-space density transiently. In conclusion the following procedure is suggested to compress $\sim 5 \times 10^{6}$ atoms to a density in phase-space of $\rho \sim 10^{-4}$. One first fully loads a MOT and then transiently switches $\left\{\Omega^{2} / \delta \Gamma, b\right\}$ to roughly $\left\{0.3,40 \mathrm{Gcm}^{-1}\right\}$ to produce the maximum spatial compression, $\sim 10^{11} \mathrm{~cm}^{-3}$, in roughly the position damping time. The idea is then to switch to parameters that will give a temperature of $3 \mu \mathrm{K}$, and cool before the density has time to decrease. The timescale for the latter is $\tau_{\text {pos }} / 3$ which might be only a few milliseconds at a high field gradient, and this may be comparable to the time required to cool (in theory cooling ought to take only $\sim 100 \mu \mathrm{s}$ but during our measurements it was always observed to take much longer than this). Thus it would be sensible to first reduce the field gradient rapidly back to its starting value prior to cooling. 


\subsection{Concluding summary}

In summary, chapters 2 and 4 have presented an empirical model of the magneto-optical trap which is a useful guide to identifying how the trap will behave under a variety of experimental conditions. The model helps to identify which experimental conditions lead to the highest phase-space densities in the trap. The model is based on a large number of experimental findings, which have been presented.

The temperature in optical molasses, obtained by switching off the magnetic field of the MOT, was measured for a range of values of the laser detuning and intensity, and agreement was found with previous studies.

The radius of the atom cloud was measured when the number of trapped atoms was low. This enabled the spring constant $\kappa$ to be deduced when the laser beam intensity was fairly high; lower limits for the spring constant were obtained from traps P1 and $\mathrm{P} 2$. There is a range of about a factor 10 in previously reported measurements of $\kappa$ (shown in Table 2.1); the Oxford value is near the upper end of this range. These measurements lead to a value for $\kappa_{0}$, the first of the three parameters in the model.

The model predicts that when sufficient atoms are loaded into the MOT, the distribution in position and in velocity will consist of a dense, cold, central region, surrounded by a relatively diffuse and hot region. This limits the number of atoms that can be confined at high phase-space density. This effect was observed under a variety of experimental conditions and confirmed the qualitative description provided by the model. The observations include measurements of the distribution of atomic velocity as well as position. A value was found for $C_{l}$, the second parameter of the model.

The density of the MOT was measured under a range of different conditions and used to deduce the third parameter in the model, $C_{\mathrm{MS}}$. A major observation was that the density in the MOT varies much more slowly with laser detuning and intensity than one would expect on the basis of a simple model of multiple scattering of photons in the trapped cloud.

It was deduced that the multiple scattering process limits the phase-space density mainly by its influence on the spatial density of the atoms, rather than by increasing the temperature. The phase-space density falls below the prediction of the model for a light shift parameter $\Omega^{2} / \delta \Gamma$ below about 0.1 . This is caused by a reduction of the spatial density, rather than an increase of the temperature. It indicates that the confinement process in the MOT breaks down before the sub-Doppler cooling mechanism does.

Evidence has been cited that the maximum density one can obtain in a MOT depends on the exact optical arrangement used to construct the MOT. The Oxford trap and trap P1 used three retroreflected laser beams, whilst trap P2 used six laser beams divided outside the vacuum system and directed independently to form the three standing waves of the MOT. While the first two traps gave similar densities, the third gave densities about two times higher under equivalent conditions.

The highest phase-space density that was observed was roughly $\rho=n \Lambda^{3}=1.5 \times$ $10^{-5}$. This is about a factor 10 below the values reported in [25], a fact which we have not been able to explain satisfactorily.

The densities that were observed were small enough that the MOT could be con- 
sidered to be in quasi-equilibrium at any time, even in its compressed phase. However, a factor 10 increase in the density would bring the MOT into the regime where the dynamics due to cold collisions will be as fast as the equilibration time for atomic position. These collisions would then be the factor limiting the density in a compressed MOT. 


\section{Chapter 5}

\section{Measurement of cold collision losses}

\subsection{Introduction}

It became evident during the study of MOT density that information about cold collisions can be inferred from the behaviour of a standard caesium vapour cell MOT. This was somewhat surprising because at typical vapour cell pressures one expects collisions with the background vapour to swamp all evidence of cold collisions induced by the trapping field (as explained in Sec. 2.4). This chapter describes the effects observed of cold collisions and explains how they were used to extract values for the cold collision loss coefficient $\beta$. The measurements were conducted at a vapour pressure $\sim 10^{-8}$ Torr, with a high MOT density (greater than $10^{10} \mathrm{~cm}^{-3}$ ) and with trap numbers in excess of $10^{6}$ atoms. By comparison, experiments measuring similar loss rates have in the past used the more complicated setup of a beam-loaded trap in order to reach background vapour pressures as low as $10^{-10}$ Torr, and have worked specifically with trapped clouds of low density and thus small numbers of atoms (typically less than $10^{4}$ ). The differences between the two approaches will be discussed.

The obvious manifestation of cold collisions is trap loss. Experiments measuring such loss rates can be broadly divided into two categories: those that have measured the rates inherent to the trap, i.e. collisions induced by the trapping laser itself $[70,74,87]$, and those that have measured the increase in loss induced by an additional laser field (the catalysis laser) $[74,42,41,67]$. The use of the catalysis laser to investigate cold collision dynamics was described in chapter 2. During the study of MOT density we were interested in the possibility that some densities were limited by collisions, and were thus concerned only with cold collision rates inherent to the caesium MOT.

The layout of the chapter is as follows. First there is a description of the manner in which cold collisions were found to modify the trap loss rate, and the method for deducing $\beta$ is explained. Evidence is then presented to confirm that the effects were indeed the result of cold collisions. The chapter proceeds with a general description of the experimental and data analysis procedure, and compares the approach here with that employed traditionally to measure cold collision rates induced by a trapping light 
field. Finally, measured values of $\beta$ in caesium are presented and discussed.

\subsection{General technique}

To extract $\beta$ from a trap loss rate one must separate its contribution to the total loss from that due to collisions with the background vapour. Traditionally one measures $\beta$ due to the MOT trapping intensity by measuring the loss rate in the temperaturelimited regime. In this regime the trap density varies (decreases) with the number of atoms and so the loss rate per atom due to cold collisions $\beta n(\mathrm{r})$ also varies with number. In contrast the loss rate per atom due to background collisions $\tau^{-1}$ is independent of number. Thus the two collision mechanisms are distinguishable by the time dependence of a trap decay. The MOT is loaded from a thermal beam which is subsequently shuttered, and the decay of atoms with time is then monitored when the trap number is sufficiently small for the volume to remain constant in time. Because the density in the temperature-limited regime is small $\left(10^{9} \mathrm{~cm}^{-3}\right.$ or less $)$ it is necessary to have a very low vapour pressure in order that $\tau$ is large enough for the two rates to be sufficiently different.

Clearly the above procedure is not applicable to a vapour cell MOT with a pressure of order $10^{-8}$ Torr; one cannot switch off the loading mechanically, and under usual conditions there are far too many atoms for the trap to be temperature-limited (of course these two are related). In the multiple scattering regime the density is nominally independent of the number of atoms and therefore so is the cold collision loss rate per atom. Thus for a normal vapour cell MOT one expects that the two types of collision process are indistinguishable. In fact it was found that this is not quite true. As remarked in chapter 4 it was observed that when the parameter values of a fullyloaded MOT were transiently switched to optimize the phase-space density, the decay of the trap number was never quite exponential, as one would expect it to be if both $\tau$ and $\beta n$ were strictly independent of number. At early times the number decayed faster than at later times, and the reason for this was that the density always decreased with number. This behaviour of the number and density is apparent in Fig. 4.12. The weak decrease of density with number is probably due to some degree of temperature-limited regime behaviour.

With the behaviour described above one finds $\beta$ from knowledge of $N(t)$ and $n(t)$ in the following way. When the trapping parameters are switched the number decay is described by Eq. (2.33). Assume first that the trap is made sufficiently weak that the loading rate $R$ may be considered to be zero, i.e.

$$
\frac{\mathrm{d} N}{\mathrm{~d} t}=-\frac{N}{\tau}-\beta \int n^{2}(\mathbf{r}, N) \mathrm{d}^{3} \mathrm{r}
$$

The last term in this equation may be expressed in terms of the peak spatial density, $n_{\max }$ :

$$
\frac{\mathrm{d} N}{\mathrm{~d} t}=-\frac{N}{\tau}-k \beta N n_{\max }
$$


where the factor $k$ is a constant that depends on the shape of the density distribution:

$$
k=\frac{\int n^{2}(\mathbf{r}) / n_{\max } \mathrm{d}^{3} \mathbf{r}}{\int n(\mathbf{r}) \mathrm{d}^{3} \mathbf{r}}
$$

For a Gaussian profile $k=2\left(2 / \pi^{3}\right)^{1 / 2} \approx 0.5$. Both $N$ and $n_{\max }$ are functions of time and thus the general solution to Eq. (5.2) is

$$
N(t)=\text { constant } \times e^{-t / \tau-k \beta \int n_{\max }(t) \mathrm{d} t} .
$$

Thus to find $\beta$ one numerically integrates the measured density with respect to time and optimizes $\beta, \tau$ until Eq. (5.4) matches the measured variation $N(t)$. One sees from Eq. (5.2) that this procedure is equivalent to measuring the decay rate at two different values of $N, n$ and solving simultaneously for $\beta, \tau$.

To be able to distinguish accurately the contributions $\beta n(t)$ and $\tau^{-1}$ to the trap decay it was often necessary to monitor a very large change in number (sometimes as much as three orders of magnitude) in order that a sufficiently large variation in density was recorded. However at small number $\left(N \lesssim 5 \times 10^{4}\right)$ the decay was observed to tend towards a small steady-state trap which is not accounted for by Eq. (5.4) because the loading rate $R$ has been neglected. If one includes $R$ in Eq. (5.1) then Eq. (5.4) becomes

$$
N(t)=I_{1}(t)\left(\text { constant }+R I_{2}(t)\right)
$$

where

$$
\begin{aligned}
& I_{1}(t)=e^{-t / \tau-k \beta \int n_{\max }(t) \mathrm{d} t} \\
& I_{2}(t)=\int I_{1}^{-1}\left(t^{\prime}\right) \mathrm{d} t^{\prime}
\end{aligned}
$$

The last term on the right-hand side is the steady-state number that the decay tends towards at long times. (For example, for the case where the density is independent of number, the solution at long times is as expected $N=R \Gamma^{-1}$ where $\left.\Gamma=\tau^{-1}+k \beta n\right)$.

Fig. 5.1 shows a typical non-exponential number decay, the corresponding decrease with time of the density and a fit to the number of Eq. (5.5) with $\beta, \tau, R$ and the overall scaling floated. The contribution to the decay from the non-zero value of $\beta$ is quite clear. Specific procedure for obtaining and analyzing these curves will be described in Sec. 5.4.

\subsection{Confirming the interpretation of cold collisions}

It was important to verify that the effects observed were indeed the result of cold collisions. Verification was sought in three independent ways.

First, the effect on the non-exponential decay of increasing the cold collision rate with far-detuned (catalysis) laser light was investigated. Light of several milliwatts at a red detuning of $125 \mathrm{MHz}$ from the $F=4 \rightarrow 5^{\prime}$ transition was generated by locking a titanium-doped sapphire laser to the crossover peak between the $F=4 \rightarrow 4^{\prime}$ and 


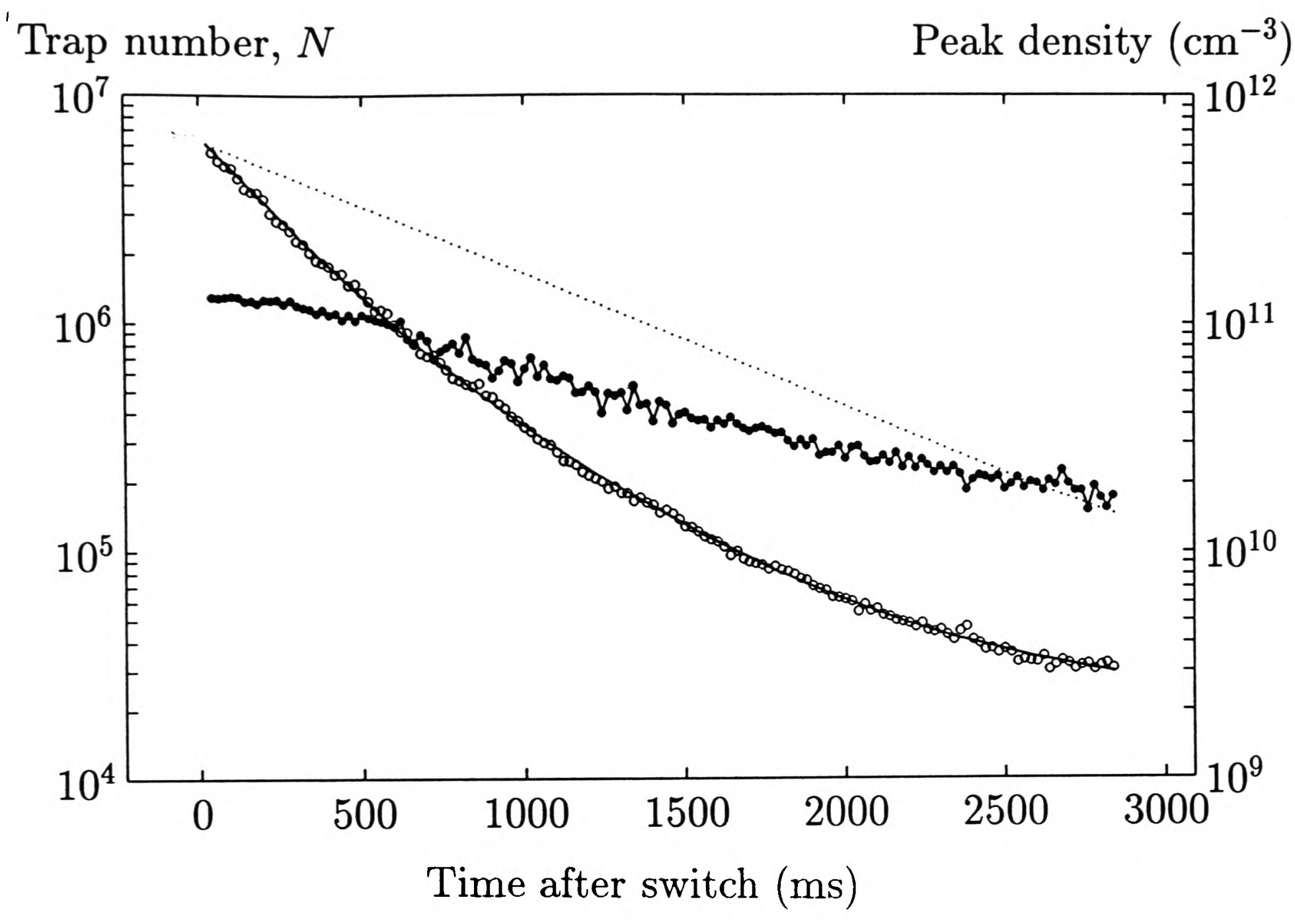

Figure 5.1: Fitting a non-exponential decay. In this example the MOT was fully loaded and then switched to the parameter values $\delta=7.66 \Gamma, \Omega=1.1 \Gamma$ and $b=40 \mathrm{Gcm}^{-1}$. In the subsequent decay the density $(\bullet)$ decreased slowly, inducing the non-exponential shape in the decay of the trap number $(0)$ that is clearly observed. The solid line is a least squares fit of Eq. (5.5) with $\beta, \tau, R$ and the overall scaling floated, and yields $\beta=1.6 \times 10^{-11} \mathrm{~cm}^{3} \mathrm{~s}^{-1}$ and $\tau=0.72 \mathrm{~s}$. The dotted line is the decay for this same value of $\tau$ but with $\beta=0$, and shows the relative contributions to the total decay from cold collisions and collisions with the background vapour.

$F=4 \rightarrow 5^{\prime}$ peaks in the saturated absorption spectrum. The light was directed down a monomode fibre, then collimated to a diameter of about $10 \mathrm{~mm}$ and aligned over the trap position. Indication that the additional laser field was indeed increasing the collision rate was immediately apparent by a large drop in the steady-state trap number for catalysis intensities of a few milliwatts. That is, $\beta$ was increased by the catalysis light such that $\beta n$ was comparable to or less than $\tau^{-1}$. Quantitative confirmation of this is shown in Fig. 5.2 which is interpreted as follows. For a trap with uniform density the steady-state number is $N=R \Gamma^{-1}$. If $N_{0}$ is the number without any catalysis laser, and if one writes $\beta$ as the sum of the coefficient due to the trapping light, $\beta_{0}$, and that induced by the catalysis laser $\Delta \beta$, then one obtains

$$
\Delta \beta=\left(\frac{1}{k n \tau}+\beta_{0}\right)\left(\frac{N_{0}-N}{N}\right) .
$$

Figure 5.2 shows that $\left(N_{0}-N\right) / N$ was proportional to the catalysis laser intensity 


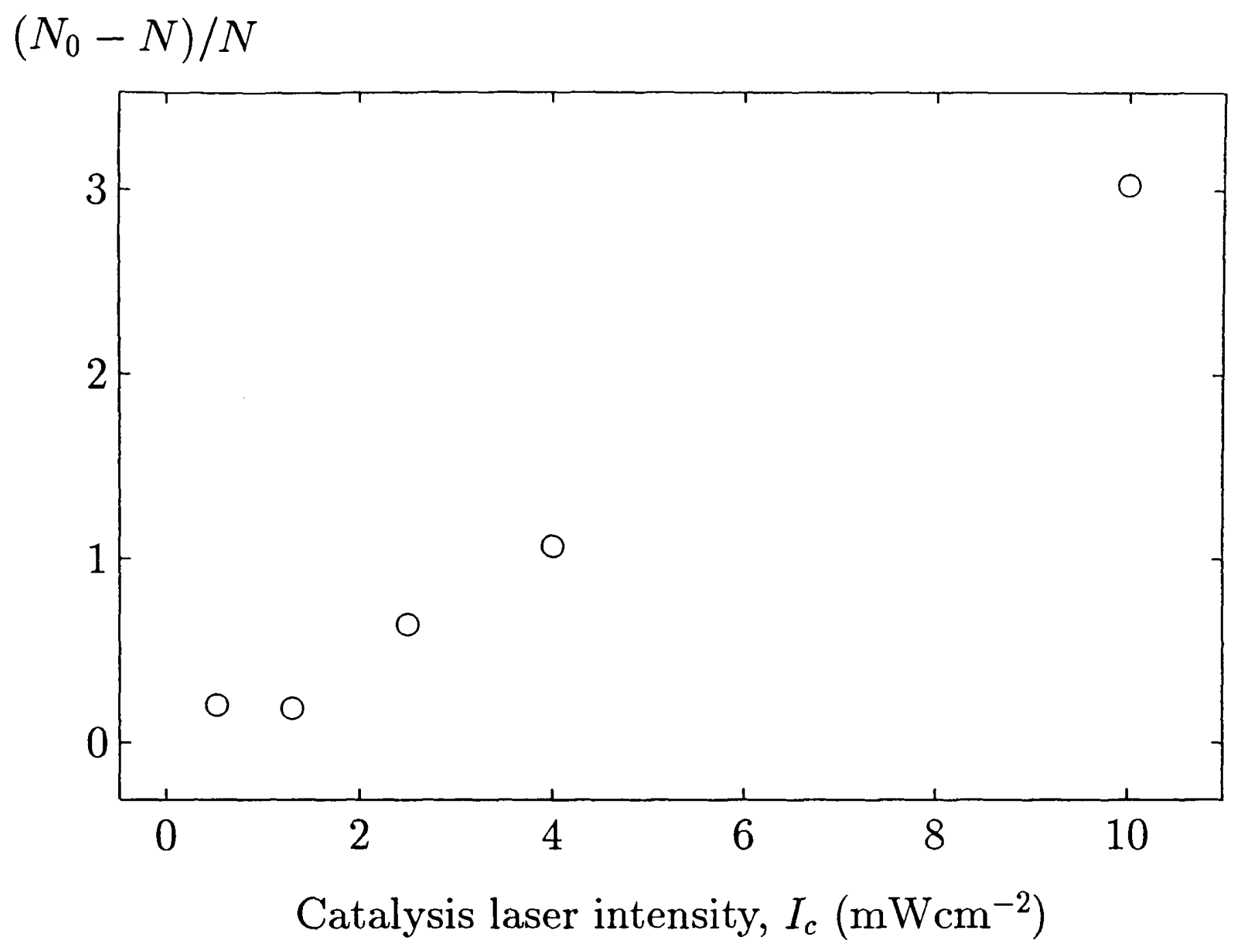

Figure 5.2: Confirmation that an additional light field of large detuning increases the light-assisted collision rate. The data shows that $\left(N_{0}-N\right) / N$ is proportional to the catalysis laser intensity, which by Eq. (5.8) implies that the induced collision rate is also proportional to the laser intensity. This is what one expects for light-assisted collisions.

$I_{c}$ and thus one concludes from Eq. (5.8) that $\Delta \beta \propto I_{c}$. This is what one expects below saturation for light-assisted collisions (Sec. 2.4.2.3). Having deduced that the far-detuned light was indeed increasing the cold collision rate, the crucial observation was that when the MOT parameters were transiently switched the effect of the catalysis light was to increase the non-exponential shape at the start of the decay.

A second idea was to test the results of the fitting procedure by taking advantage of the very different spatial densities that exist in the two-component density regime. One expects that if $\beta n$ is comparable to $\tau^{-1}$ in the central, strongly-confined region of a two-component profile, then in the outer, weakly-confined area $\beta n \ll \tau^{-1}$ because of the factor $\sim 100$ difference in density. Thus one can imagine of the following test. If the MOT is switched to parameters that give a two-component profile and the decay rate of the atoms that are weakly-confined is measured, then this rate should be roughly the same as the value of $\tau^{-1}$ deduced in the manner described above from the non-exponential decay of the central component. (They might not be exactly the same because the large spatial spread of the weakly-confined atoms may reduce their 
average escape velocity). Unfortunately it turned out to be infeasible to implement this idea fully. Under the conditions necessary to just enter the two-component regime the decay rate due to cold collisions was only comparable to or less than that due to background collisions, and this made it difficult to test the fitting procedure. In other words, it was not easy to resolve the non-exponential component of the decay. The reasons for this were that (a) a low intensity is required to obtain a two-component profile (Sec. 4.6) and this reduces the value of $\beta$ due to light-assisted collisions, and (b) the loss rate due to background collisions increases faster with decreasing trap depth than one would expect (further evidence for this will be presented in this chapter to complement that already given in chapter 6). However, under these circumstances the strongly-confined atoms did indeed decay at roughly the same rate as those weaklyconfined, as expected. An interesting observation was made when the trapping was weakened to push the atoms further into the two-component regime. At a certain point the decay rate of the central region increased rapidly whereas that of the surrounding region did not. In Sec. 4.6 this was cited as evidence for the onset of hyperfine structurechanging collisions contributing to loss, causing a considerably larger loss rate in the central region because the density there is much higher.

For a third approach the dependence of $\beta$ on the total trapping intensity was measured and compared to a previous measurement [74]. This data is presented and discussed in Sec. 5.5.

\subsection{Discussion of method}

This section discusses the procedure adopted to maximize the usefulness of the technique presented. An explanation of the shortcomings of the method is given, including a discussion of the factors limiting the accuracy, and a comparison is drawn with the traditional method used to measure $\beta$ induced by the MOT light field.

The first point to make is that since one is looking to distinguish a decay rate $\beta n(t)$ against a background rate $\tau^{-1}$ it is advantageous to switch the parameter values to those that give large densities. From chapter 4 it is clear that one almost attains the maximum MOT density simply by increasing the magnetic field gradient sufficiently. For loss measurements this is an ideal means to increase the density because one is then free to measure $\beta$ as a function of intensity and detuning. All measurements were performed by transiently increasing the field gradient to $40 \mathrm{Gcm}^{-1}$. A second key point is that the method presented relies entirely upon the density varying with number in the multiple scattering regime. This is a weak variation. To separate the cold collision rate from the background collision rate accurately one requires the density to change by, say, an order of magnitude, and as demonstrated by Fig. 5.1 this generally means recording the number decay over some two to three orders of magnitude. Herein lies a difficulty. Unless the parameters are switched to values that give very weak trapping, the decay will tends towards a significant steady-state trap and therefore limit the range over which the number varies. For this reason measurement was limited to a saturation parameter low enough for the final steady-state number to be small $\left(<10^{5}\right)$ yet still large enough to permit detection. For example, to measure $\beta$ at high intensity 
$\left(I_{t o t}>30 \mathrm{mWcm}^{-2}\right)$ it was necessary to switch to $\delta>7 \Gamma$. It can now be appreciated that increasing the field gradient gave the additional advantage of weakening the trap, thereby relaxing the extent to which the trap needed to be weakened by increasing the detuning or reducing the intensity. It should be pointed out that conveniently it was precisely the values of $\beta$ at low scattering rates that were relevant to collision-limited densities. Finally one should realize that as far as the background collision rate is concerned, weakening the trap by reducing the scattering rate is to some extent offset by the fact that the excited state population is small and thus interaction is via the weak van der Waals force. Typically by switching to the parameter values described above the product $k \beta n \tau$ at $t=0$ was roughly three, and this was fairly independent of scattering rate.

It is instructive to make a comparison of the above performance with a measurement of $\beta$ in the temperature-limited regime. From figure 1 in reference [74] $\beta=7.6 \times$ $10^{-11} \mathrm{~cm}^{3} \mathrm{~s}^{-1}, n_{\max }(t=0)=1.2 \times 10^{9} \mathrm{~cm}^{-3}$ and $\tau=132 \mathrm{~s}$ thus implying $k \beta n \tau \approx 6$. Therefore for detecting the effects of $\beta$ the two methods have comparable starting points, with low background pressure traded for higher density. However, clearly in the temperature-limited regime the density decreases much more rapidly with number, and this means that to achieve the same level of unambiguity in the determination of $\beta$ and $\tau$ one can afford to monitor the decay over a smaller change in number. In the multiple scattering regime one is forced to monitor over a much longer decay, which is eventually curtailed by the formation of a small steady-state trap, even for very weak trapping forces. This extra complication in the decay introduced a certain amount of ambiguity in the fitting, particularly in determining $\tau$ which is found from the behaviour at long times. Because $\beta$ is determined mainly by the shape of the decay curve at short times it was found to be somewhat less sensitive to the exact shape of the decay at long times. Finally, measuring $\beta$ in the multiple scattering regime does have the distinct advantage that with a much larger number of atoms signals at low scattering rates are considerably stronger.

In summary it is suggested that the technique we have developed has the advantages of working in a simple vapour cell and allowing $\beta$ to be determined with some reasonable degree of accuracy at low saturation parameters. This was perfectly sufficient for the investigation of collision-limited density. To obtain accurate measurements at standard trapping parameters a measurement in the temperature-limited regime is probably more applicable.

In the light of the above discussions the following steps were taken to give the best experimental accuracy. The volume of the MOT was never deduced from a single camera alone, but always measured with two cameras. These were used with complementary aperture settings to extend the dynamic range of detection to up to three orders of magnitude. In addition particularly weak profiles were fitted over a cross of narrow width rather than a grid in order to reduce the amount of unwanted noise being summed into the profiles. When the width of this cross was narrower than the trap width itself the total number of atoms had to be calculated from the fit parameters rather than by integration of the total signal. The signal-to-noise ratio of the image was also improved by summing profiles from several fields before fitting; this tended to 
be necessary only at long times where the loss in time resolution from such averaging was unimportant. A crucial part of the experimental setup was the intensity-locking system described in chapter 3 ; this ensured constant intensity to better than about $5 \%$ over a period of $10 \mathrm{~s}$ after switching.

\subsection{Measurements of $\beta$}

The method outlined was used to measure the dependence of $\beta$ on the total trapping intensity. The reasons for choosing this particular measurement were three-fold. First, comparison with previous measurements of the intensity dependence $[74,87]$ would provide confirmation of the measuring technique. Secondly, only the intensity dependence can reveal which particular cold collision mechanism is dominant at a given intensity. Finally, one obtains the full picture of the absolute magnitude of $\beta$ from which deductions about collision-limited densities can be made.

An example of the variation of $\beta$ with trapping intensity is shown in Fig. 5.3(a) and was obtained by switching the parameter values to $\delta=7.66 \Gamma, b=40 \mathrm{Gcm}^{-1}$ and to the desired intensity. The corresponding values of the lifetime $\tau$ against background collisions are shown in Fig. 5.3(b). Comment should first be made on the accuracy of the data. In general the fractional random scatter of the $\beta$ values is comparable to that of the caesium measurements in [74], although considerably worse than those of the rubidium measurements in [87]. Thus the method certainly served the original aim of allowing qualitative assessment of $\beta$. For intensities greater than $40 \mathrm{mWcm}^{-2}$ the loading rate $R$ was significant enough to prevent the number decaying below $2 \times 10^{5}$ atoms, and $\beta$ could not be accurately found. For the same reason values of $\tau$ were not accurately determined for intensities greater than $30 \mathrm{mWcm}^{-2}$. At low intensities the large values of $\beta$ were generally well determined, but at the very lowest intensities $\left(<6 \mathrm{mWcm}^{-2}\right)$ the image signals were low and the decays very quick $\left(\Gamma^{-1}<100 \mathrm{~ms}\right)$ and to find $\beta$ it was necessary to fix $\tau$ according to an extrapolation of Fig. 5.3(b). In light of this discussion the following uncertainties are suggested. Random error was typically $\sim 30 \%$ except at the very lowest intensities where it was $\sim 50 \%$. A value of $\beta$ was found only to the absolute accuracy of measuring the density, and this was previously estimated to be $\sim 50 \%$.

Both curves in Fig. 5.3 divulge interesting information. First, one notices reproduction of the distinctive variation of $\beta$ described in Sec. 2.4.2.3. At low intensity the trap is very weak and the kinetic energy released in a hyperfine structure-changing collision is sufficient to overcome the trap depth and induce loss. The resulting loss rate is large. As the intensity is increased the trap deepens and these collisions are eventually suppressed, leaving only light-assisted collisions. The figure suggests that the rate of light-assisted collisions increases linearly with trapping intensity.

The solid line through the $\beta$ values is a least squares fit to the function $\beta=$ $A_{1} e^{-I_{\text {tot }} / I_{\tau}}+A_{2} I_{\text {tot }}$ and gives $I_{\tau}=1.1 \mathrm{~mW}, A_{2}=1.2 \times 10^{-12} \mathrm{~cm}^{5} \mathrm{~s}^{-1} \mathrm{~mW}^{-1}$. As explained in Sec. 2.4.2.3 the fact that $\beta$ does increase linearly with intensity is evidence for the dominance of fine-structure changing collisions rather than radiative redistribution. There are two further pieces of evidence to support this conclusion. First, very 


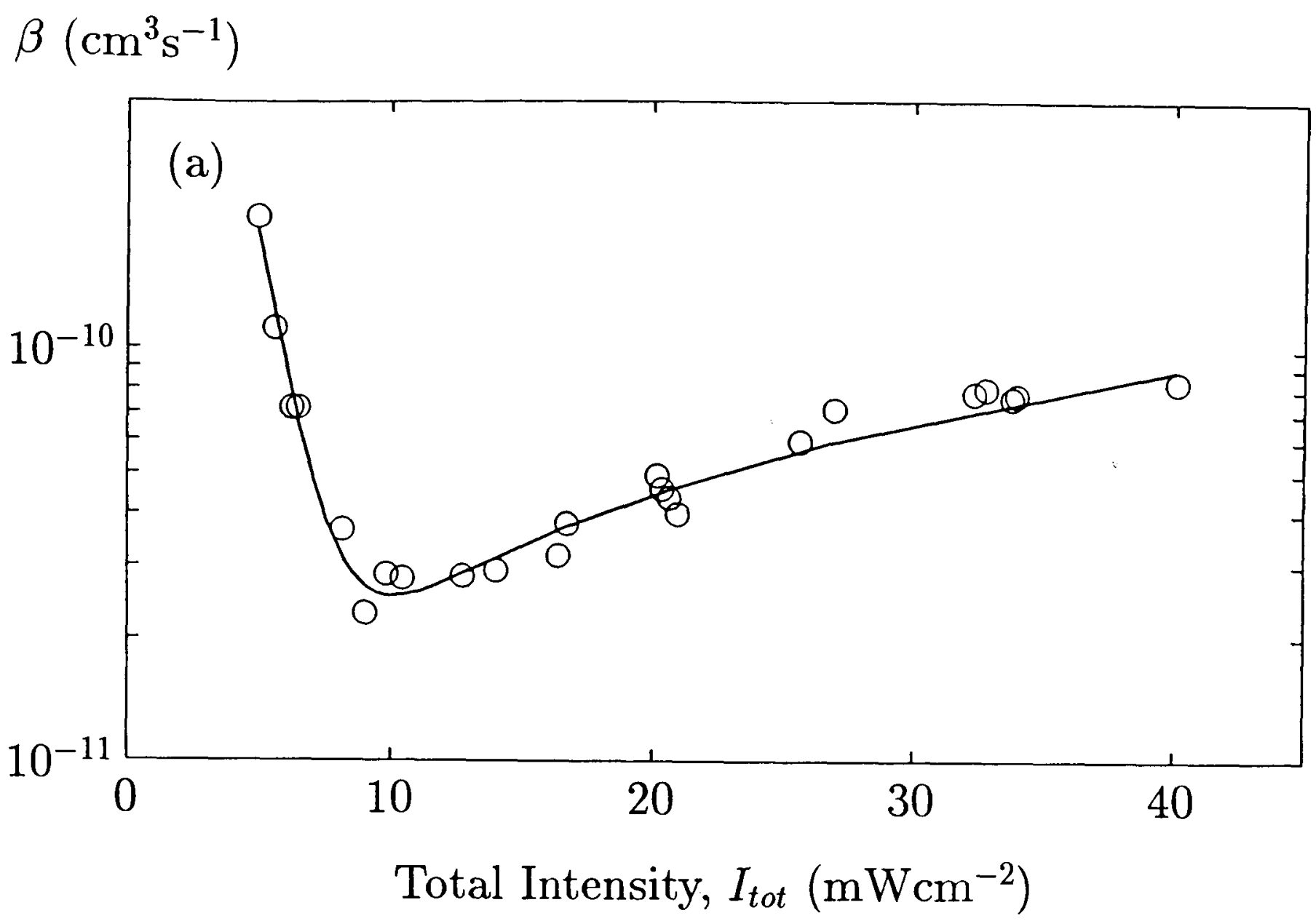

$\tau(\mathrm{s})$

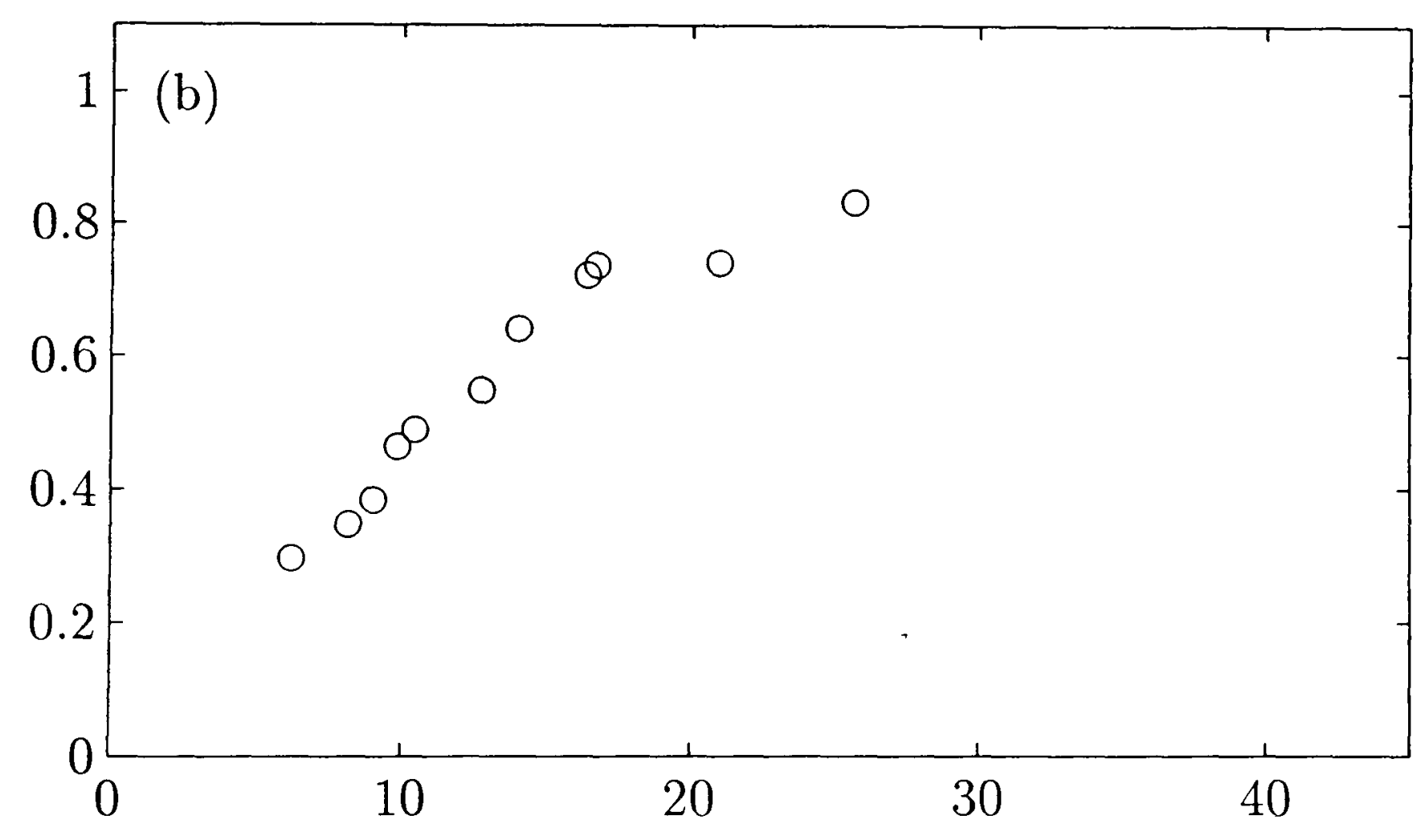

Total Intensity, $I_{t o t}\left(\mathrm{mWcm}^{-2}\right)$

Figure 5.3: Variation of (a) the cold collision coefficient and (b) the lifetime against background collisions with total trapping intensity. The data was obtained by switching the MOT to the parameter values $\delta=7.66 \mathrm{\Gamma}, b=40 \mathrm{Gcm}^{-1}$ and to the chosen intensity. The solid line through the $\beta$ values is a least squares fit to the function $\beta=A_{1} e^{-I_{\text {tot }} / I_{\tau}}+A_{2} I_{\text {tot }}$ where the two terms represent respectively the extinction of hyperfine structure-changing collisions and the linear dependence of light-assisted collisions with intensity. 
similar curves to that shown were obtained over a period of three months and encompassing several different trap alignments (although it was noticed, as in [87], that when the trap was poorly aligned $\beta$ did not vary significantly with intensity, presumably because the trap was sufficiently leaky for hyperfine structure-changing collisions to make a significant contribution to the loss at all intensities). Radiative redistribution varies with trap depth and thus, one would expect, alignment. Secondly, the linear dependence was also observed in caesium by Sesko et al. [74]. However, despite this convincing evidence one should perhaps refrain from concluding unambiguously that radiative redistribution is insignificant in caesium because (a) there are no definite predictions of how quickly the rate should vary with trap depth, and"(b) its effect at low intensity is potentially swamped by the turn-on of hyperfine structure-changing collisions.

The values of $\beta$ can be compared directly with those of Sesko et al. [74]. In the region where light-assisted collisions are dominant measurements at common intensities are in agreement to within a factor of two. In addition, the straight line gradients agree to about $20 \%$. Measurements in [74] were performed with $\delta \approx \Gamma$ whereas those of Fig. 5.3 were at $\delta=7.66 \Gamma$. Thus one infers that the light-assisted collision rate has not varied significantly with this detuning over this small range. This is in agreement with the adapted version of fig. 3 of [74] republished in [42], but is at odds with the GallagherPritchard model [c.f. Eq. (2.50)]. The largest values of $\beta$ are due to hyperfine structurechanging collisions, with a maximum in excess of $2 \times 10^{-10} \mathrm{~cm}^{3} \mathrm{~s}^{-1}$, in agreement with [74] to within the experimental uncertainties discussed above. These maximum rates were quoted in chapter 4 and combined with measurements of the position damping time to conclude that none of the MOT densities observed were close to the collision limit.

Fig. 5.3(b) shows that the background collision rate increases nearly linearly with decreasing trapping intensity at low intensities. Together with the deductions made from observations of the dark SPOT (Secs. 6.4.3 and 6.4.4.1) this is evidence to suggest that the background collision rate for a weak trap is underestimated by a classical calculation. According to Eq. (2.37) a classical expression for $\tau$ varies as $v_{e s c}^{1 / 3}$ for the van der Waals interaction. Thus Fig. 5.3(b) implies that $v_{e s c} \propto I_{\text {tot }}^{3}$. This rapid variation of the escape velocity with intensity is not remotely predicted by a consideration of Doppler slowing forces in the capture volume [56]. A possible reason for this failure was cited in chapter 2. Although the collisions involve many partial waves, diffraction effects are significant if the de Broglie wavelength of the momentum transfer is comparable to the interaction distance. A quantum mechanical calculation of the differential angular cross-section is generally finite in the forward scattering direction for a potential of the form $C_{n} / r^{n}$, but the classical expression is divergent. Thus for traps with depths comparable to the energy transfer at which the quantum and classical descriptions differ significantly, the latter will tend to exaggerate the likelihood of a glancing collision leading to heating rather than loss. The quantum effects of high-energy glancing collisions have been addressed previously $[65,3,59]$. 


\subsection{Concluding summary}

In conclusion a method was devised that permitted cold collision rates to be measured in a regime where one might expect the effect of background collisions to be overwhelming, and without the need for the more complicated procedure of loading from an atomic beam which is subsequently shut off. The accuracy of the technique was comparable to previous measurements in caesium and the values of $\beta$ obtained show that the MOT densities reported in chapter 4 were not collision-limited. 


\section{Chapter 6}

\section{The caesium dark MOT}

\subsection{Introduction}

The measurements presented in chapter 4 confirm that the highest phase-space density attainable in the MOT is between four and five orders of magnitude short of quantum degeneracy. Other trapping techniques permitting greater compressions or cooling to lower temperatures are therefore of great interest. One such scheme is the dark MOT or dark SPontaneous force Optical Trap (dark SPOT), which was proposed by Ketterle and demonstrated by his group [51] to give a significant improvement over standard magneto-optical trapping. Sodium densities approaching $10^{12} \mathrm{~cm}^{-3}$ were reached by modifying the ordinary MOT in such a way that the atoms were confined mainly in a hyperfine level outside the main cooling cycle. In this "dark" hyperfine level atoms are isolated from the undesirable effects of the trapping light. For example, in the multiple scattering regime the reabsorption of scattered light is significantly reduced, alleviating the density-limiting and heating effects described in chapter 2.

The importance of the dark MOT, however, lies not just in the improved phasespace density it can yield; indeed we will show that in caesium the phase-space densities are usually lower than those in the MOT. Its value in providing conditions of high density and large trapping numbers for efficient evaporative cooling has recently been demonstrated. Atoms trapped in a dark MOT have been loaded into magnetic traps and evaporatively cooled $[69,23]$, and this has led to the observation of BEC in rubidium [1]. The realization of a caesium dark MOT could be similarly significant in the effort to observe collective quantum effects in caesium.

This chapter describes the application of the dark MOT to caesium. Before this work was started, only the study by Ketterle et al. in sodium [51] had reported improved compression with a dark MOT. Notably, the investigation of the rubidium dark MOT by Anderson et al. [2] made no mention of higher densities. During this work, therefore, it was important to appreciate the similarities to and the differences from the situation with much lighter sodium. A summary of the findings to be presented is as follows. Significantly, we demonstrated that it is possible to achieve high densities of $\sim 10^{12} \mathrm{~cm}^{-3}$ in the dark MOT of a heavy alkali. This compression represents almost an order of magnitude improvement on the best density that we were able to attain 
in the conventional MOT. However, we find that in a static trapping scheme the large hyperfine splitting of caesium makes the implementation of the technique much less straightforward than in sodium. It will be shown that an unfortunate consequence of this complication is a heating of the static dark MOT substantially above the molasses temperature, resulting in a phase-space density lower than that attainable in the caesium MOT. A simple model for the dark MOT density is discussed and its main assumptions investigated by Monte-Carlo simulation; the adequacy of this model for accounting for the observed density behaviour under the conditions we have used is verified. In a vapour cell arrangement [66] more atoms are trapped in a static dark MOT than in a similar MOT by virtue of a reduced cross-section for collisional loss. Anderson et al. [2] showed that at very low vapour pressures $\left(\lesssim 10^{-10}\right.$ Torr $)$ this effect can be significant, indeed it was crucial to the degenerate quantum conditions they were able to attain by evaporative cooling [1]. At the moderate vapour pressures we have used $\left(\sim 10^{-8}\right.$ Torr $)$ the increase in number is a smaller effect. Measurements are presented to illustrate this and are used to deduce information about the crosssection for collisions between trapped atoms and the background vapour. Finally, it is demonstrated that forming the conditions of a dark MOT temporally also permits high densities to be attained, but without the additional heating that is unavoidable in the spatial configuration. However, the trapping times are observed to be extremely short due to collisional losses. From the density dependence of the trap decay it is deduced that the collisional losses are from both cold collisions processes and trapped atoms-background vapour collisions. These fast decays render the trapping scheme impractical and are themselves a density-limiting mechanism.

This chapter is organised in the following way. In Sec. 6.2 the dark MOT scheme is fully explained. A simple model describing the trapped density, number and temperature is considered and justified by a Monte-Carlo simulation of the basic processes. The experimental setup for the realization of both spatial and temporal dark MOT is described in Sec. 6.3. Section 6.4 records the experimental investigations and compares them with the theory presented.

\subsection{The dark MOT}

The distinction between loading a large number of atoms and creating a high phasespace density is of crucial importance for understanding the operation of both the MOT and the dark MOT. The total number is predominantly determined by the capture process, i.e. the slowing of individual atoms by the Doppler cooling forces in the intersection region of the laser beams. The density and temperature, however, are determined by the polarization-gradient forces at the trap centre. The conditions for loading the maximum number of atoms do not give the optimum phase-space density. It was for precisely this reason that the technique employed in chapter 4 was a two-step process of first loading the MOT with a large number of atoms by using a large scattering force, and then subsequently increasing the detuning or field gradient or decreasing the intensity to produce cooling and compression. This transient procedure is of particular importance for caesium and rubidium because the temperature 
corresponding to the recoil limit for these heavy atoms, which can be approached by the polarization-gradient cooling mechanisms, is over two orders of magnitude below the Doppler cooling limit. Thus there is a large advantage in using parameters which optimize the sub-Doppler mechanisms.

In the MOT, therefore, one reduces the undesirable effects of scattering by direct reduction of the scattering rate. In the dark MOT the equivalent procedure is to shelve atoms into the dark level, and thus reduce the time spent in the cooling cycle. The concept of a two-stage process to reach high phase-space densities applies equally to the dark MOT. Significantly, the original sodium experiment [51] illustrated that the scattering conditions for the trapped atoms (i.e. their degree of confinement to the dark level) can be altered whilst simultaneously maintaining the high loading rate of the MOT. This is possible because the trapped atom cloud is localized near the trap centre but the capture of atoms occurs throughout the whole volume of the trapping beam intersection. Advantage can thus be taken of the spatial separation of loading and trapping. We term this trapping scheme the spatial dark MOT. (In principle, one could do the equivalent for the MOT simply by reducing the intensity of the trapping beams at the trap position whilst maintaining a high intensity for efficient loading of atoms around the outside of the beams). As pointed out by Ketterle, a transient variation of the conditions is also perfectly possible. One can load a large number of atoms into a MOT and then switch the conditions to those of a dark MOT, in a similar manner to the transient procedure usually used in the MOT. We call this scheme the temporal dark MOT. Investigations of both the spatial and temporal dark MOT in caesium will be presented.

\subsubsection{Population transfer}

In a caesium MOT light at $852 \mathrm{~nm}$ excites the highest frequency component of the D2-line, corresponding to the transition from the upper hyperfine level in the ground state to the upper hyperfine level in the excited state, the $F=4 \rightarrow 5^{\prime}$ transition. Spontaneous decay takes the atoms back to the original level, forming a closed cooling cycle, as marked on Fig. 6.1(a). Trapping and cooling have been observed for other transitions [71] but the forces are generally much weaker [79].

In the alkali atoms the cooling cycle is not completely closed. Off-resonant excitation to the excited level one lower than that of the cooling cycle leads to decay to the lower hyperfine ground state. In caesium this is the transition sequence $F=4 \rightarrow 4^{\prime} \rightarrow 3$. The leakage out of the cooling cycle is normally counteracted by a repumping laser beam which excites atoms out of the lower level on a transition which enables them to decay back into the upper ground state level. Ketterle exploited this feature of the alkalis in a novel way: by reducing the intensity of the repumping laser sodium atoms were allowed to accumulate in the lower level, leaving only a small fraction in the cooling cycle. This procedure is very straightforward in sodium, but for heavier alkalis the excited state hyperfine splitting is larger and the off-resonant excitation rate is much smaller. Thus to transfer the same population to the dark level the repumping rate much be reduced much further. It is difficult to do this in the 


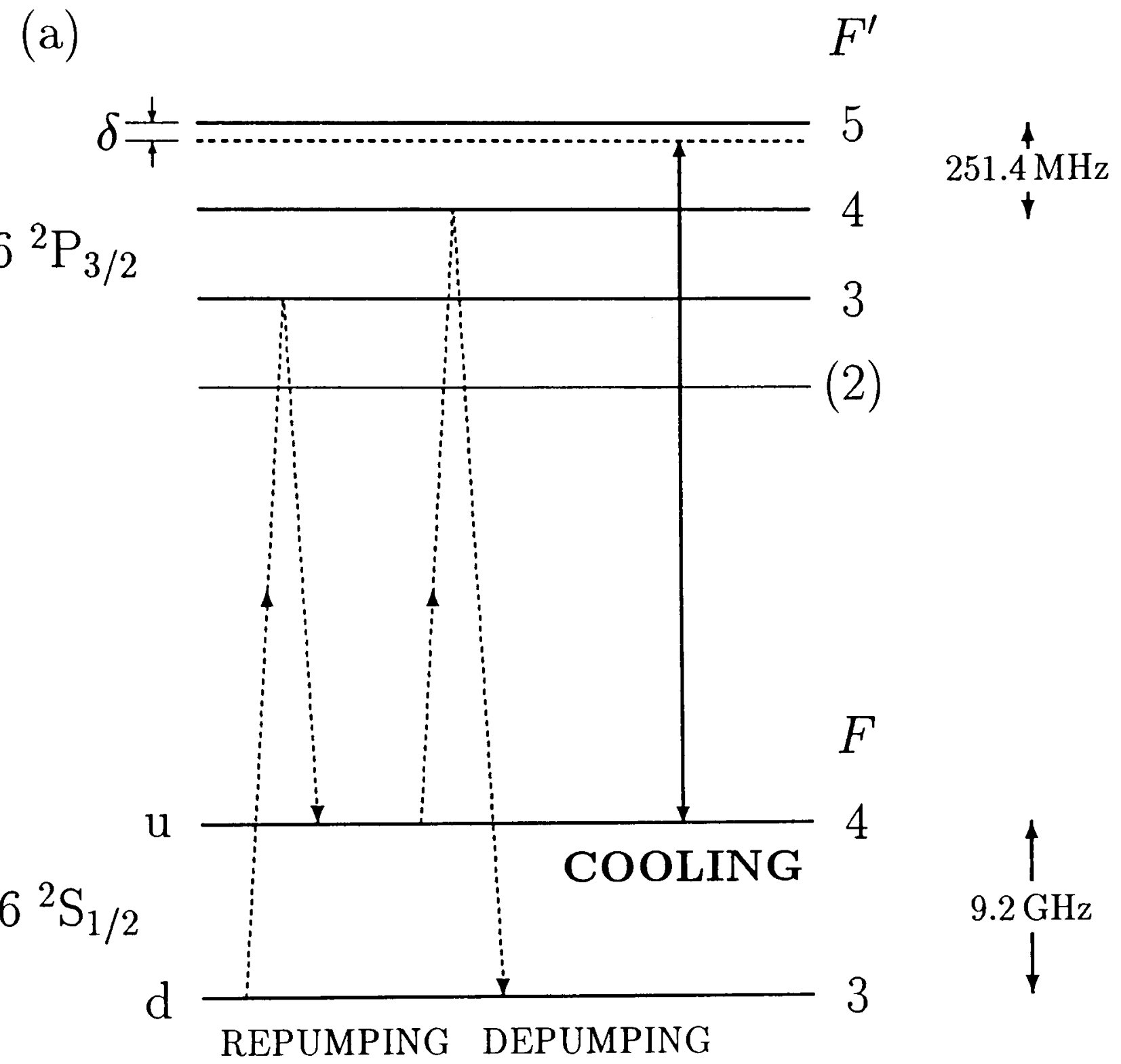

(b)

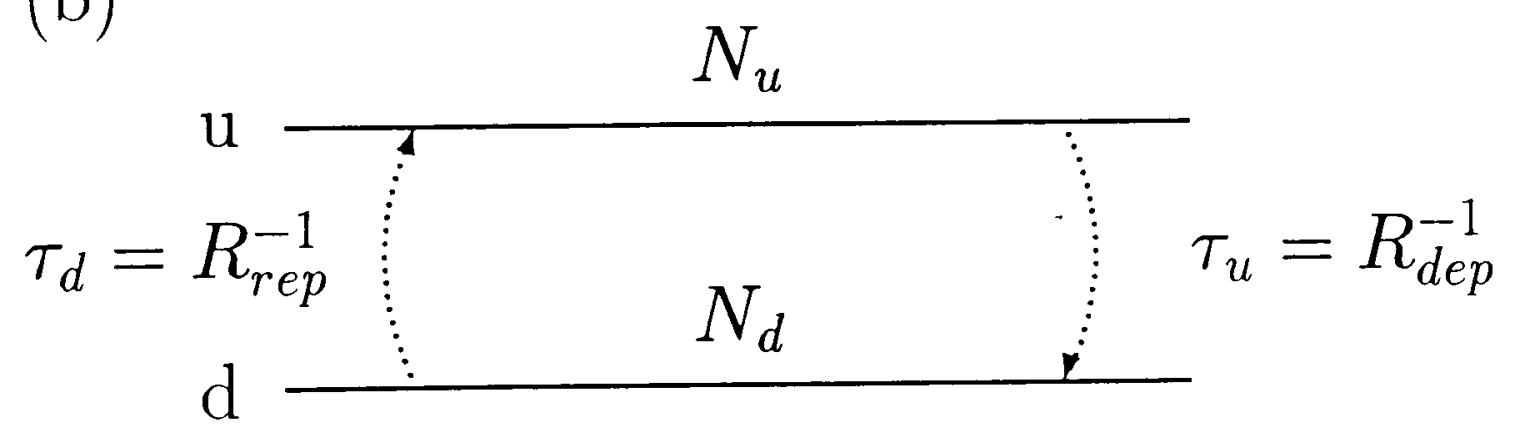

Figure 6.1: (a) Energy levels and relevant transitions in the D2 resonance line of cesium and (b) control of populations using repumping and depumping. 
spatial dark MOT. In this steady-state trapping scheme the repumping is kept large around the outside of the trapping volume in order to maintain a high loading rate, whilst in the centre it is reduced by imaging a "hole" in the repumping beam at the trap position (see Sec. 6.3). Although the imaging significantly reduces the repumping intensity at the centre, small amounts of stray light are scattered into the trap from the capture region outside and these are generally intense enough to prevent a large population accumulating in the dark level. For this reason it is essential to have another laser frequency which actively pumps atoms down into the lower level. This has been referred to as "depumping" [51] and is indicated by the $F=4 \rightarrow 4^{\prime}$ transition in Fig. 6.1(a).

For simplicity in the following description, the repumping light is considered to be resonant with the $F=3 \rightarrow 3^{\prime}$ transition, as indicated in Fig. 6.1(a). This transition avoids the same upper level as the depumping light and thus removes the complication of considering Raman processes. (Alternatively, the $F=4 \rightarrow 3^{\prime}$ transition could be used for depumping and $F=3 \rightarrow 4^{\prime}$ for repumping with slight changes to the intensities to take account of the relative line strengths). In fact we have evidence that such coherent effects are not particularly significant under the conditions we have used (Sec. 6.4.1), but their complete neglect allows treatment of the populations in the upper and lower hyperfine levels with rate equations.

The essential features of our treatment of the dark MOT are illustrated in Fig. 6.1(b). In the upper hyperfine level of the ground state, or "bright state", $N_{u}$ atoms interact with the trapping light, and for low magnetic fields their behaviour is similar to that in optical molasses, with a temperature damping time $\tau_{m o l}$. The lifetime of atoms in this upper state before being depumped into the lower hyperfine level of the ground state is $\tau_{u}$, and this is determined by the intensity of the depumping laser, with an upper limit set by the off-resonant excitation rate. Atoms remain in the lower level or "dark" level for a mean time $\tau_{d}$ before they are excited by the weak repumping light back into the cooling cycle. The equilibrium number in the dark level is $N_{d}$.

If $R_{r e p}$ and $R_{d e p}$ are respectively the repumping and depumping rates, then clearly

$$
\frac{N_{u}}{N_{d}}=\frac{\tau_{u}}{\tau_{d}}=\frac{R_{r e p}}{R_{d e p}}
$$

To characterize how "dark" the trap is, Ketterle et al. [51] used the parameter $p$ to denote the fraction of atoms in the bright state:

$$
p \equiv \frac{N_{u}}{N_{u}+N_{d}}
$$

We will assume that the presence of the depumping light does not interfere with the sub-Doppler forces generated on the $F=4 \rightarrow 5^{\prime}$ cooling cycle. This assumption is good if the population excited by the depumping light is small compared to that excited by the trapping light, and this is true for the depumping intensities we have used. It is then useful to think of $p$ as the ratio of the dark MOT fluorescence to the MOT fluorescence when switching rapidly from one to the other. We often deal with large 
fractions in the dark state in which case $p$ is small and adequately approximated by

$$
p \approx \frac{\tau_{u}}{\tau_{d}}
$$

The value of $p$ is adjusted by change of the depumping and repumping rates. However, if the dark MOT is to yield its largest phase-space density then there are temperature constraints on the minimum value of $\tau_{u}$ and possible density constraints on the maximum value of $\tau_{d}$. The reasons for this are explained in the following sections.

\subsubsection{Dark MOT density}

We now present a simple model for the dependence of the dark MOT density on the parameter $p$. Of the density-limiting processes described in chapter 2 we will be concerned mainly with the multiple scattering and temperature-limited regimes. In the dark MOT the fraction of time an atom spends on the $F=4 \rightarrow 5^{\prime}$ cooling transition is given by the factor $p$. With the above assumption that the presence of depumping light is only a small perturbation on the cooling process, the concepts of a spring constant $\kappa$ and friction coefficient $\alpha$ are still valid. The time spent on the cooling cycle before being depumped into the dark level is short compared to the position damping time in the trap, $\alpha / \kappa$. Therefore, as far as motion in the trap is concerned, the time-averaged spring constant is reduced by the factor $p$.

In the multiple scattering regime the density is expected to be proportional to the spring constant (Sec. 2.2.2), but the repulsion between atoms from the reabsorption of scattered light is a two-body event and its rate will thus depend on the square of the fraction of atoms in the cooling cycle, i.e. proportional to $p^{2}$. Then in this regime the overall dependence of the dark MOT density is

$$
n_{M S}^{d M O T}=\frac{n_{M S}^{M O T}}{p}
$$

where the subscript "MS" indicates that this equation applies to the multiple scattering regime only. Clearly there is an increase in density when $p<1$. One may conveniently interpret the form of Eq. (6.4) as only that fraction of atoms which remains in the cooling cycle as being subject to the density-limiting processes that occur in the MOT.

In the temperature-limited regime the MOT radius $r_{T}$ is determined by the spring constant and the cloud temperature $T$, the three being related by the equipartition theorem:

$$
\frac{1}{2} \kappa r_{T}^{2}=\frac{1}{2} k_{B} T
$$

The spatial distribution is close to Gaussian and, assuming the spring constant to be isotropic, the peak density with $N$ atoms is [from Eq. (2.6)]

$$
n_{T}^{M O T}=\frac{N}{\left(\sqrt{2 \pi} r_{T}\right)^{3}} .
$$


Thus in this regime the dark MOT density decreases as $p$ is reduced:

$$
n_{T}^{d M O T}=\frac{N}{(\sqrt{2 \pi})^{3}}\left(\frac{\kappa}{k_{B} T}\right)^{3 / 2} p^{3 / 2}
$$

Here $T$ is the temperature in the dark MOT and we shall indicate later under what circumstances this will differ from the temperature in the MOT.

There is a third density-limiting mechanism whose effect is enhanced as $p$ is reduced. The atoms in the dark state travel undamped for a mean time $\tau_{d}$ and with an rms velocity $v=\sqrt{k_{B} T / m}$. The mean distance they travel in a given direction is then of the order of $\lambda=v \tau_{d}$ and so broadening of the trap dimensions on this scale might be expected. To estimate this effect we can use $\lambda$ to define a density in a similar manner to Eq. $(6.7)^{1}$. This gives

$$
n_{\lambda}=\frac{N}{(\sqrt{2 \pi})^{3}}\left(\frac{m}{k_{B} T}\right)^{3 / 2}\left(\frac{p}{\tau_{u}(1-p)}\right)^{3} .
$$

We refer to this regime as a "free travel-limited" density. It is clear that this process will only be significant if the time spent in the dark state is sufficiently long, i.e. if a given $p$ is attained by means of low repumping and low depumping rather than high repumping and high depumping. Thus the effect is inherently largest in the heavy alkalis where the natural depumping rate is small.

Figure 6.2 shows how the dark MOT density varies with $p$ in the three regimes described above, according to Eqs. (6.4), (6.7) and (6.8). Roughly speaking, the actual density at each $p$ is given by that expression with the smallest value ${ }^{2}$. The trapping conditions for each plot have been chosen to illustrate the free travel-limiting effect at small $p$ and low repumping intensity, and the importance of trapping a large number of atoms for attaining high densities. Common to each plot are a laser detuning $\delta=2 \Gamma$, a Rabi frequency per beam $\Omega=\Gamma$ and a field gradient $\mathrm{d} B / \mathrm{d} z=15 \mathrm{Gcm}^{-1}$. These are trapping conditions not very dissimilar to those that might be used for loading the trap. Values for other parameters under these trapping conditions have been estimated from the measurements presented in chapter 4 . The spring constant has been derived from Eq. (2.8) with the measured value $\kappa_{0}=10^{-19} \mathrm{Nm}^{-1}$. A molasses temperature of $60 \mu \mathrm{K}$ has been estimated from Fig. 4.3, and the MOT $(p=1)$ density has been taken to be $3 \times 10^{10} \mathrm{~cm}^{-3}$.

In Fig. 6.2(a) the upper state lifetime has been made negligibly small which, by Eq. (6.3), also implies that the time spent in the dark level can be neglected and the effect of free travel thus ignored. This corresponds to the case of strong forced depumping. By contrast, the depumping in Fig. 6.2(b) is only that which is naturally

\footnotetext{
${ }^{1}$ The exact shape of the profile caused by undamped travel in the dark level is addressed in Sec. 6.2.5.

${ }^{2}$ One could argue that the density is more accurately represented by some sort of sum of the contributions from the three regimes, e.g. radii added in quadrature. This is particularly relevant at the small values of $p$ where the two volume-limiting processes dominate, because their effects will clearly add independently. However, the aim of this model is to predict a qualitative trend for the density and to this end refinements of this sort can be ignored.
} 

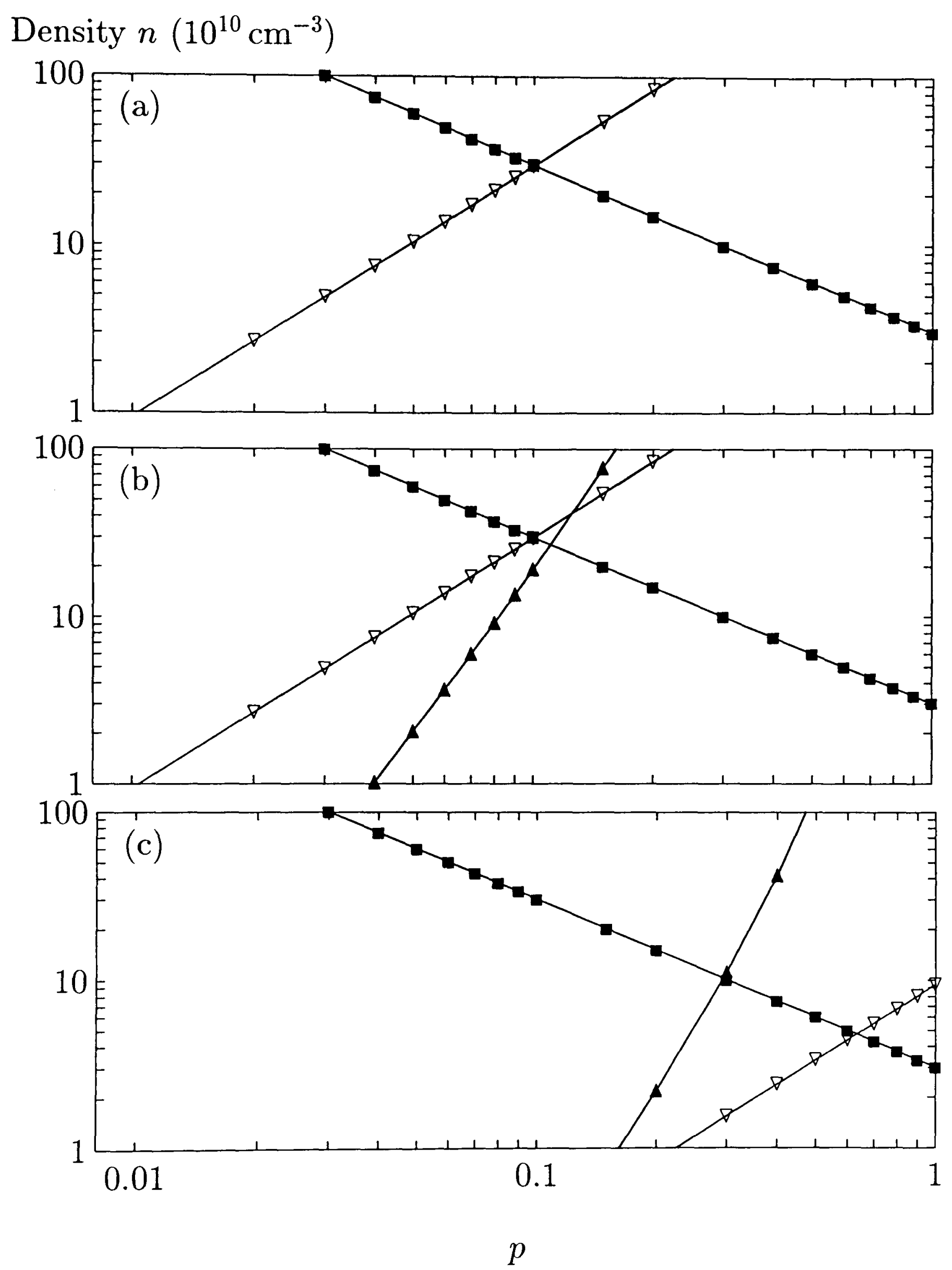

Figure 6.2: Trap regimes as a function of the density and the fraction in the bright state $p$. The boundaries are : multiple-scattering density, $\nabla$ : Temperature-limited density, $\mathbf{\Delta}$ : Free travel-limited density. The trapping conditions for each plot are described in the text. Values for the spring constant, molasses temperature and MOT density have been estimated from the measurements in chapter 4. 
present, i.e. there is no depumping laser. If $\Delta$ is the hyperfine splitting in the upper excited state between the $F^{\prime}=5$ and $F^{\prime}=4$ levels, then a rate equation estimate of this small off-resonant excitation rate is

$$
R_{d e p}^{n a t}=\frac{\Gamma}{2} \times \frac{6 \Omega_{44^{\prime}}^{2} / 2}{(\Delta+\delta)^{2}+6 \Omega_{44^{\prime}}^{2} / 2+\Gamma^{2} / 4} \times \frac{S_{4^{\prime} 3}}{S_{4^{\prime} 3}+S_{4^{\prime} 4}}
$$

where the factors of 6 account for the intensity from six trapping beams and the last term is the line strength weighting for the decay $F^{\prime}=4 \rightarrow 3$ to the lower hyperfine ground state. The Rabi frequency $\Omega_{\mathbf{4}^{\prime}}$ for the transition $F=4 \rightarrow 4^{\prime}$ is related to that for the $F=4 \rightarrow 5^{\prime}$ cooling cycle by a further ratio of line strengths:

$$
\frac{\Omega_{44^{\prime}}^{2}}{\Omega^{2}}=\frac{S_{44^{\prime}}}{S_{45^{\prime}}}=\frac{21}{44} .
$$

The hyperfine splittings in caesium are much greater than the natural linewidth and hence much greater than the Rabi frequency when close to saturation. Therefore,

$$
\begin{aligned}
R_{d e p}^{n a t} & \approx \frac{\Gamma}{2} \times \frac{6 \times(21 / 44) \Omega^{2} / 2}{(\Delta+\delta)^{2}} \times \frac{15}{36} \\
& \approx 0.3 \Gamma \frac{\Omega^{2}}{(\Delta+\delta)^{2}}
\end{aligned}
$$

which, with the above trapping parameters, gives a lifetime in the upper state of $\tau_{u}=1 / R_{d e p}^{\text {nat }} \approx 250 \mu \mathrm{s}$. This lifetime is insensitive to the trap detuning because $\Delta \gg \delta$.

The trap number in Fig. 6.2(a) and Fig. 6.2(b) has been chosen to be $10^{7}$, which is realistic for the trapping conditions stated under typical vapour cell loading. Fig. 6.2(c) differs from Fig. 6.2(b) by having the substantially smaller number of $5 \times 10^{5}$ atoms. In all three plots it has been assumed that the trap number remains constant as $p$ is varied. Although more atoms are loaded into a spatial dark MOT as $p$ is reduced, the effect is small unless vapour cell pressures are very low, typically less than $10^{-10}$ Torr (see Sec. 6.2.4 and Sec. 6.4.3).

The plots reveal some interesting features for the conditions we have considered. They suggest that the general effect of undamped motion in the dark level is to lower the density at small $p$ and cause some small reduction of the maximum density. Necessarily this is a qualitative observation because of the very simplified way Eq. (6.8) has converted the effect of free travel into a limiting density. It is clear that under the conditions presented, the weakening of the spring constant in the temperature-limited regime will be the dominant factor limiting the maximum density, but that this limit can be elevated to a higher density by trapping more atoms. Similar improvements are possible by the use of a higher field gradient (to increase the spring constant) or by cooling to a lower temperature (to reduce the temperature-limited volume). However, both of these techniques may be difficult to implement in a steady-state dark MOT because the number of trapped atoms decreases dramatically with increasing field gradient [45], and the use of forced depumping heats the trap (Sec. 6.2.3). 


\subsubsection{Temperature and phase-space density}

There are several temperature considerations in a dark MOT. First, the excess heating in the MOT from the reabsorption of scattered photons (Sec. 2.2.2) is a two-body event and will therefore decrease as $p^{2}$ in the dark MOT. Secondly, strong depumping can heat the trap. A depumping rate large enough to reduce the lifetime in the upper ground state to less than the molasses damping time causes the coherence between the ground state sublevels in the sub-Doppler cooling mechanisms to be reduced. Drewsen et al. [25] measured the molasses damping time in caesium to be about $40 \mu \mathrm{s}$. This value was fairly insensitive to the trap detuning because both the induced orientation and Sisyphus cooling mechanisms contribute to the damping in a MOT, and their variations tended to cancel. A time of $40 \mu \mathrm{s}$ is consistent with our measurements of the depumping intensity required to heat molasses (Sec. 6.4.1). It is also several times smaller than the estimate in the previous section of the upper state lifetime against natural depumping, which is consistent with the observation of sub-Doppler temperatures in the caesium MOT. A third temperature effect is suggested by the Monte-Carlo simulation described in Sec. 6.2.5. An excess heating in the dark MOT which increases as $p$ is reduced is predicted. This is a result of a coupling between the velocity and position of atom caused by its travel in the dark level. However, its magnitude has not been predicted for realistic trapping forces.

In the caesium spatial dark MOT the small natural depumping rate makes the use of a depumping laser essential for attaining small $p$. From the temperature measurements to be presented in Sec. 6.4.1 the depumping intensity required to reduce $p$ to the value $\sim 0.1$ where a maximum in the density might be expected (Fig. 6.2), is that which is expected to heat the trap above its molasses temperature by a factor $\sim 2-3$. This is what was observed (Sec. 6.4.4). This heating clearly limits the usefulness of the spatial dark MOT as a technique in itself for attaining high phase-space densities. However, this shortcoming can be avoided by application of a cooling scheme after the high density has been attained. For example, one could change the dark MOT conditions back to those of the MOT and then reduce the scattering rate to lower the temperature. Because the temperature damping time in the MOT is much shorter than the position damping time, this cooling can be achieved before the density has had a chance to decrease. Alternatively a dark state cooling scheme could be applied directly to the dark MOT (see [29] for example).

High densities can be obtained in a temporal dark MOT without forced depumping; it is then possible to simultaneously combine a large compression with a molasses temperature. However, in anticipation of the results presented in Sec. 6.4, and in a behaviour similar to that described in chapter 4 for the MOT, the spatial confinement of the atoms in the dark MOT is observed to decrease when the light shift parameter falls below about $\Omega^{2} / \delta \Gamma \sim 0.2$. Therefore, as in the MOT, the highest densities are not compatible with the lowest achievable molasses temperatures of a few microkelvin. For this reason it seems reasonable to suggest that the caesium dark MOT improves upon the phase-space density attainable in the caesium MOT by the factor of a higher density at the point of breakdown of confinement. It should be pointed out that the improved density we have obtained implies a phase-space density which is still an order 
of magnitude below that reported by Drewsen et al. [25] in an ordinary caesium MOT, a fact which we have not been able to explain satisfactorily.

\subsubsection{Trapped numbers and lifetimes}

As mentioned in Sec. 6.1, a possible motivation for using a spatial dark MOT is to reduce the collisional loss rate from the trap, thereby increasing the number of atoms that can be trapped. The expression for the equilibrium number of atoms loaded from a background vapour at typical pressure was given in chapter 1:

$$
N=C\left(\frac{v_{c}}{\bar{c}}\right)^{4} \frac{d^{2}}{\sigma}
$$

In a small trap the Zeeman shifts are comparable to the linewidth and it is sufficient simply to assume that the stopping distance is similar to that in Doppler molasses with beams of the same diameter $d$. The capture velocity then has the dependence $v_{c} \propto d^{1 / 2}$, and thus for fixed trapping parameters the trapped number depends on the fourth power of the trap dimensions, $N \propto d^{4}$. Increasing the beam size is then the most straightforward method to load a large number. For example, with $60 \mathrm{~mm}$ diameter beams Gibble et al. [33] loaded $10^{10}$ atoms into a MOT. Therefore, the emphasis in our investigation of the dark MOT has not been to improve trapped numbers. We have taken a given number and concentrated on maximising its density. However, in this section we consider how measurements of the equilibrium number of atoms in a spatial dark MOT can be used to determine relative collision cross-sections with the background vapour.

The cross-section for collisions leading to the loss in Eq. (6.13) can be written as

$$
\sigma=\rho_{g g} \sigma_{g}+\rho_{e e} \sigma_{e}
$$

$\rho_{g g}$ and $\rho_{e e}$ are the fractional populations in the ground and excited states respectively and in a two-level atom approximation are given by the usual density matrix treatment [57]:

$$
\begin{aligned}
& \rho_{e e}=\frac{1}{2} \times \frac{\Omega_{t o t}^{2} / 2}{\delta^{2}+\Omega_{t o t}^{2} / 2+\Gamma^{2} / 4} \\
& \rho_{g g}=1-\rho_{e e}
\end{aligned}
$$

where $\Omega_{\text {tot }}=\sqrt{6} \Omega$ is the total Rabi frequency. Atoms in the excited state have a strong dipole-dipole interaction with ground state atoms of the same species. Their cross-section for collisions with the background vapour is, therefore, larger than for the atoms in the ground state, which interact only via the weaker van der Waals force. That is, $\sigma_{e}>\sigma_{g}$. In the dark MOT, the fraction $p$ of atoms that are in the cooling cycle interact with the cross-section Eq. (6.14), whilst the remaining fraction $(1-p)$ in the dark level only undergo ground state collisions. It then follows that the ratio of dark MOT to MOT equilibrium numbers is

$$
\frac{N_{d M O T}}{N_{M O T}}=\frac{1+\rho_{e e}\left(\sigma_{e} / \sigma_{g}-1\right)}{1+\rho_{e e}\left(\sigma_{e} / \sigma_{g}-1\right) p} .
$$


Clearly $N_{d M O T}>N_{M O T}$ because $p<1$, which is simply a consequence of the dark MOT having a larger fraction of atoms interacting via the weaker van der Waals force.

Under loading conditions, where typically $\rho_{e e}$ might be about 0.3 , the classical estimate by Steane et al. [77] discussed in Sec. 2.4 .1 predicts $\sigma_{e} / \sigma_{g} \sim 6$. For this case the simple treatment above predicts a number ratio of about 2.5 in the limit of small $p$; this is close to what was observed experimentally (Sec. 6.4.3). One might expect this number ratio to remain relatively constant as the scattering rate is reduced by some moderate amount because, as $\rho_{e e}$ decreases, the prediction by Steane et al. [77] is that the ratio of cross-sections varies with the trap escape velocity $v_{e s c}$ as

$$
\frac{\sigma_{e}}{\sigma_{g}} \propto v_{e s c}^{-1 / 3}
$$

which increases as the trap weakens.

Anderson et al. [2] have studied the dark MOT for rubidium in a very low-pressure vapour cell trap. At $10^{-11}$ Torr trap loss under loading conditions was dominated by light-assisted intra-trap collisions rather than collisions with the background vapour. As will be explained in Sec. 6.4.4.1, the probability of these events decreases as $p$ in the dark MOT for sufficiently large dark state fractions (see Sec. 6.4.4), and roughly an order of magnitude increase in the trapped number was observed.

\subsubsection{Monte-Carlo simulation of a dark MOT}

A Monte-Carlo simulation has been developed to investigate the physics of the dark MOT and in particular to verify the basic assumptions of the model that has been presented. This work has been undertaken by Dr. N. H. Edwards. The approach has been to use a simple one-dimensional model, and to look for qualitative trends in the simulation results. This technique has been valuable in studying the standard MOT $[56]$.

The simulation works in the following way. Doppler forces only are considered, subDoppler cooling and atom-atom interactions being neglected. This greatly simplifies the calculations without necessarily altering the basic physics. Moreover, capture and escape from the trap, which generally involve Doppler forces only, can be very usefully studied. The position and velocity of an atom is recorded as it interacts with counterpropagating $\sigma^{+}$and $\sigma^{-}$red-detuned laser beams in the presence of a linearly varying magnetic field. Photon absorption is treated as a random event but with the mean excitation rate $R_{ \pm}$from each beam fixed by the usual expression for a two-level atom:

$$
R_{ \pm}=\frac{\Gamma}{2} \times \frac{\Omega^{2} / 2}{\Omega^{2} / 2+\Gamma^{2} / 4+(\delta \pm k v \pm \mu z \mathrm{~d} B / \mathrm{d} z)^{2}}
$$

where $\mu$ is the atomic magnetic moment. Magnetic sublevels have also been ignored. In a MOT the circularly polarized beams tend to optically pump the atoms to the fully stretched states $m_{F}= \pm F$, and once an end state is reached the cooling transition behaves much like $J=0 \rightarrow 1$. Moreover, a full sublevel treatment would not necessarily makes the simulation any more realistic. In a real three-dimensional trap atoms tend to 
absorb photons from all six trapping beams and thus with respect to a fixed direction transitions are stimulated by photons of mixed polarization.

The main assumptions of the dark MOT model that has been presented are that the spring constant is reduced by the fraction of time spent in the cooling cycle $p$, and that the velocity distribution of the atoms in the cooling cycle is unaffected by the free travel in the dark level. Appealing to the one-dimensional theories of Doppler cooling and the Doppler MOT $[28,54]$ and also to the equipartition theorem, one would then expect the following analytical expressions for the dark MOT temperature-limited radius and velocity spread in the limit of low intensity saturation:

$$
\begin{aligned}
r_{T}^{2} & =v_{T}^{2} \frac{m}{\kappa}, \quad \text { where } \\
v_{T}^{2} & =\frac{2 D_{p}}{\alpha m}=\frac{\hbar \Gamma}{m} \frac{\delta^{2}+\Omega^{2} / 2+\Gamma^{2} / 4}{\delta \Gamma} \\
\kappa & =\kappa_{0} p \\
\kappa_{0} & =\frac{\mu \alpha}{\hbar k} \frac{\mathrm{d} B}{\mathrm{~d} z}=4 \mu \hbar k \frac{\mathrm{d} B}{\mathrm{~d} z} \frac{\Omega^{2}}{\Gamma^{2}} \frac{\delta \Gamma}{\left(\delta^{2}+\Omega^{2} / 2+\Gamma^{2} / 4\right)^{2}}
\end{aligned}
$$

and $D_{p}$ is the momentum diffusion coefficient, $\alpha$ is the friction coefficient and $\hbar k$ is the photon momentum.

Simulations have been carried out for a range of repumping and depumping intensities that have been experimentally realistic, and the temperature and size distributions compared to the one-dimensional expressions above. We have noticed two interesting phenomena.

First, there is a variation in temperature as a function of $p$ which is not predicted by Eq. (6.21). As shown in Fig. 6.3, the trap size corresponds well to the analytical expression close to $p=1$ but diverges as $p$ is reduced. For $0.01 \lesssim p \lesssim 0.85$ the fractional discrepancy in size increases roughly as $p^{-3 / 4}$, which implies that the temperature is increasing fractionally as $p^{-3 / 2}$ above the theoretical estimate. The reason for the discrepancy appears to be a correlation between the velocity and position of an atom caused by its travel in the dark level. Such a correlation can arise in the following way. Untrapped travel may result in an atom moving outside the trap size determined by the equipartition theorem. When repumped back into the cooling cycle, the atom is then in a "non-equilibrium" position and its velocity is damped rapidly. There then ensues a slow restoring of its position back to the cloud on the time scale of the position damping time of Doppler forces. The drift velocity of this restoration process is an excess heating. A correlation mechanism of this type is general and should occur in a real three-dimensional trap with sub-Doppler forces. However, its significance in this instance is unknown for the largest dark state fractions we have used experimentally $(p \sim 0.05)$. Suffice to say that its effect has not been observed, possibly because in the temperature-limited regime the trap temperature is observed to be dominated by the heating from the depumping light.

The second observation of interest is a change in the trap profile at low repumping intensities, providing confirmation of the presence of free travel in the dark level. When the mean free path becomes comparable to the trap size the spatial distribution of the atoms begins to exhibit a two-component structure, with the wings of the profile 


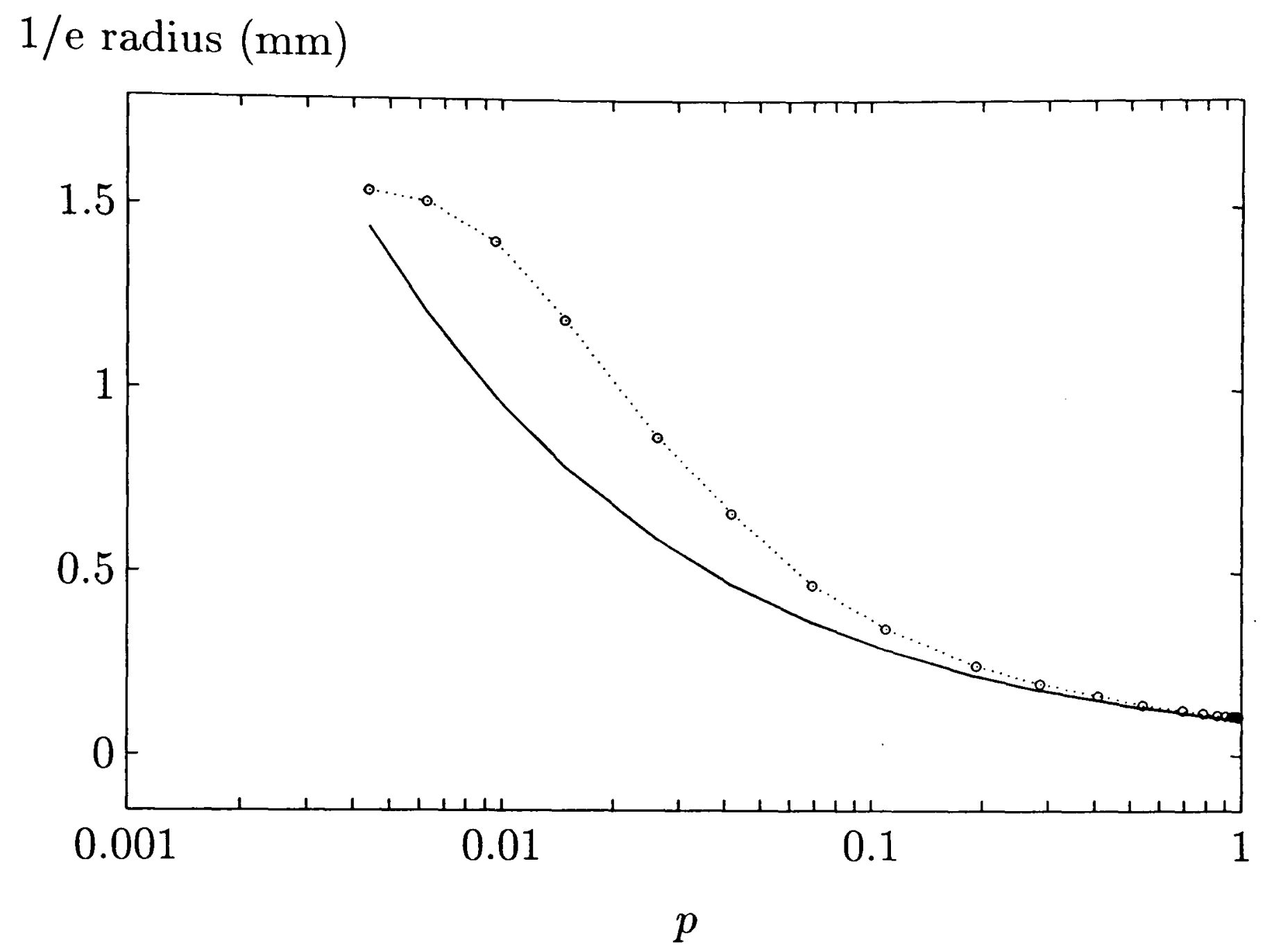

Figure 6.3: One-dimensional Monte-Carlo (o) and theoretical (-) estimates of trap radius as a function of the fraction in the bright state, $p$. The simulation agrees well with the theoretical estimate for $p$ close to unity.

extending out to large radius. This smearing of the distribution to the wings is easily understood. If atoms with a Gaussian velocity distribution of width $\Delta v$ were to each spend exactly a time $t$ in the dark level, then the profile resulting from free travel would clearly be a Gaussian of width $t \Delta v$. However, the absorption of a repumping photon in the dark level is a random event with a mean time between absorptions $\tau_{d}$. That is, the survival probability in the dark level decays exponentially with time constant $\tau_{d}$. Thus the profile resulting from the free travel is a sum of Gaussians weighted exponentially:

$$
\int_{0}^{\infty} e^{-\xi} e^{-x^{2} / \lambda^{2} \xi^{2}} \mathrm{~d} \xi \quad \text { where } \quad \xi \equiv \frac{t}{\tau_{d}} \text { and } \lambda=\tau_{d} \Delta v
$$

The shape of this integral is very similar to an exponential with decay distance $1 / \lambda$, that is, the profile is dominated by the exponential survival probability. In the temperaturelimited regime the total trap profile is roughly the convolution of this exponential with the Gaussian of radius $r_{T}$. 


\subsection{Experimental setup}

The dark MOT setup required modifications to the repumping arrangement used for the ordinary MOT and the addition of a depumping beam.

A $50 \mathrm{~mW}$ DBR laser was used as the source of repumping light, and was locked to either the $F=3 \rightarrow 4^{\prime}$ or $F=3 \rightarrow 3^{\prime}$ transitions with the arrangement described in chapter 3. The use of the AOM was essential to allow precise and fast control of the repumping intensity for the temporal formation of the dark MOT. As described in chapter 3 this control was achieved by electronic attenuation of the rf signal from a voltage-controlled oscillator driven at $100 \mathrm{MHz}$, and a lookup table and computer locking system allowed a particular repumping intensity to be quickly selected, switched and maintained.

Fast and precise control of the intensity was necessary for the temperature measurements described in Sec. 6.4.1. This was achieved with an identical setup to that for the repumping light. The depumping beam was expanded by a telescope arrangement of a pair of lenses to a diameter of about $3 \mathrm{~mm}$, which was large enough to affect the cloud of trapped atoms whilst having little influence on the capture process.

\subsubsection{The temporal dark MOT}

The temporal dark MOT was formed by reducing the repumping intensity transiently in the MOT after loading. The trap decayed quickly as a result of a much reduced trap depth, but its image was recorded transiently using the image acquisition system described in chapter 3 . The dark MOT parameter $p$ was deduced from the ratio of total fluorescence after the switch to that before.

\subsubsection{The spatial dark MOT}

The spatial dark MOT involves a more complex repumping arrangement but has the major advantage that the dark MOT is maintained in a steady state. A region of low repumping intensity surrounded by a region of high intensity was obtained by intersecting two perpendicular repumping beams each with a "hole" in the middle. The beam quality was good, since the mono-mode fibre which took the repumping light from the optical table to the vacuum system acted as a spatial filter. The collimated repumping beam passed through a microscope slide with a black spot of insulating tape stuck onto it, the "dark spot", which had a transmission of less than $10^{-4}$. The light was then split to produce two orthogonal paths propagating at $45^{\circ}$ to the trapping beams as shown in Fig. 6.4. In each path the dark spot was imaged at the centre of the trapping region using a pair of lenses of focal length $f=40 \mathrm{~cm}$ placed a distance $2 f$ apart. The microscope slide was positioned one focal length before the first lens, and the trap position was one focal length after the second lens. This optical system left the Gaussian beam unchanged (i.e. it was a telescope with unity magnification). Simply placing a spot in a beam without imaging caused Fresnel diffraction effects at the trap centre because the beam paths were necessarily long due to the large size of the vacuum chamber. 


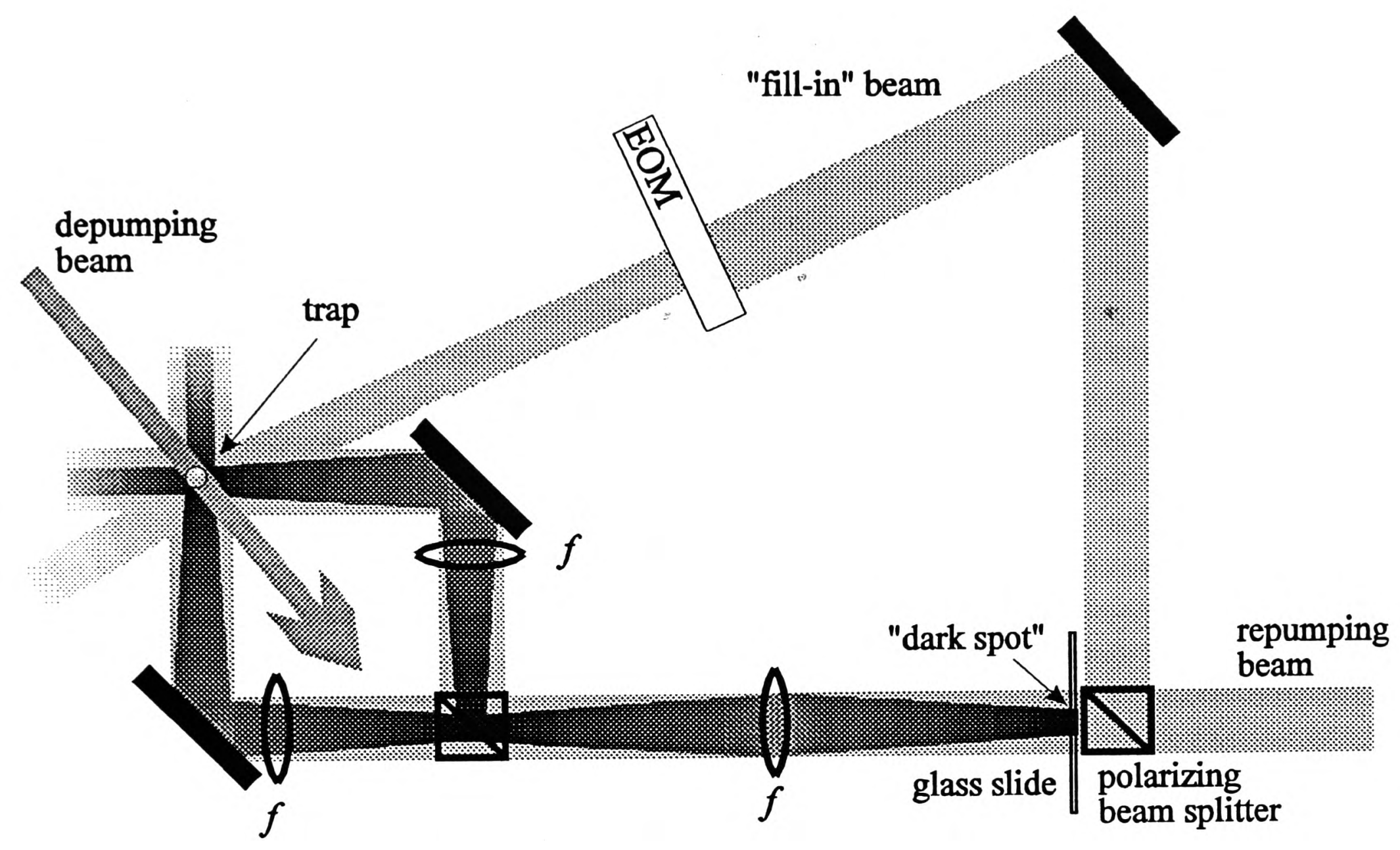

Figure 6.4: Arrangement of beams in the horizontal plane of the spatial dark MOT.

Spot sizes between $1.3 \mathrm{~mm}$ and $6 \mathrm{~mm}$ diameter were tried. Best confinement of the atoms in the "hole" (without decreasing the trap loading rate significantly) was obtained for sizes between 3.5 and $4.5 \mathrm{~mm}$. All the experiments referred to in this chapter were performed with a spot size of $3.5 \mathrm{~mm}$.

Aligning the spot with the centre of the trap proved to be simple. First it was aligned with the centre of the repumping beams which themselves were centred on the trap position. Then one beam was blocked and the trap'viewed along an axis at a small angle to the unblocked beam. The spot was translated until its darkened image was visible moving over the trap. This procedure was then repeated for the other beam. To observe the image clearly it was sometimes necessary to reduce the overall intensity of the repumping beam in order to get a large fraction of the atoms into the dark state.

Imaging the spot reduced the repumping intensity at the trap position by one to two orders of magnitude. However, this was only sufficient to reduce $p$ slightly from unity if saturation of repumping in the surrounding capture region was also maintained. To reach large dark state fractions depumping was essential. About $100 \mu \mathrm{Wcm}^{-2}$ of $F=4 \rightarrow 4^{\prime}$ light was shone into the trap and this reduced $p$ to about 0.15 . Higher values of $p$ were then obtained by means of an auxiliary beam of repumping light which illuminated the cloud directly; the intensity of this "fill-in" beam was controlled 
by an electro-optical modulator (EOM) sandwiched between two crossed polarizers (extinction ratio $\sim 200: 1$ ) and with a supply voltage controlled remotely by computer. Lower values of $p$ were reached by detuning the repumping light away from resonance by up to four linewidths (it was observed that this did not noticeably reduce the trap loading rate). Combinations of these two procedures allowed $p$ to be varied in the range of roughly $0.05-1$.

The fill-in beam was also used to measure the dark state fraction. The depumping light was switched off and the EOM switched on in order to change conditions from those of a dark MOT to those of a MOT in a few microseconds. Calibration of the fluorescence after switching gave the number trapped in the dark MOT, whilst the ratio of fluorescence before the switch to that after gave the dark MOT fraction.

\subsection{Dark MOT experiments}

\subsubsection{Effect of depumping on trap temperature}

To verify the heating effect of forced depumping (Sec. 6.2.3) the temperature of atoms in optical molasses was measured for varying amounts of $F=4 \rightarrow 4^{\prime}$ depumping light. A small number of atoms $\left(\lesssim 10^{6}\right)$ was loaded from a low vapour pressure with the trap detuning set to $-2 \Gamma$, a Rabi frequency per beam of $1.5 \Gamma$ and a magnetic field gradient of $10 \mathrm{Gcm}^{-1}$. The trapping laser detuning was then switched to $-4 \Gamma$ and its intensity reduced to a few percent of its initial value to remove any residual heating from multiple scattering. Simultaneously the field coils were switched off and the depumping light switched on to the required intensity. After a wait of a few milliseconds the trapping beams were quickly extinguished (in less than $1 \mu \mathrm{s}$ ) followed by the depumping beam $50 \mu$ s later. The repumping light was switched off with a fast shutter a few hundred microseconds after this (the heating effect from the $50 \mu \mathrm{s}$ of scattering between repumping and depumping transitions after switching off the trapping beams was estimated to be less that $20 \%$ of the total heating effect that was observed). The atoms fell the $42 \mathrm{~cm}$ under gravity to the standing-wave probe beam tuned to the resonance of the $F=4 \rightarrow 5^{\prime}$ transition, and the fluorescence from the probe was recorded as a function of time. With this large trap-probe separation any slight uncertainty in the initial trap size contributed negligibly to the uncertainty in the temperature measurement, but the effect of sideways pushing on the weakly-confined molasses by absorption of the depumping light was much exaggerated. Unless the depumping beam was reflected back upon itself the atoms fell outside the $2 \mathrm{~cm}$ wide probe beam and no TOF signal was observed.

The variation in molasses temperature with depumping intensity is indicated by the open circles in Fig. 6.5. Each data point represents an average of between one and ten shots, with the strength of the TOF signal depending on the depumping intensity. At higher intensities than those shown the loss of atoms through pushing effect of the depumping light was too large to retain a signal. For the data shown the $F=3 \rightarrow 4^{\prime}$ transition was used for repumping but very similar results were obtained when repumping on $F=3 \rightarrow 3^{\prime}$. The filled circles on the same figure are the molasses 


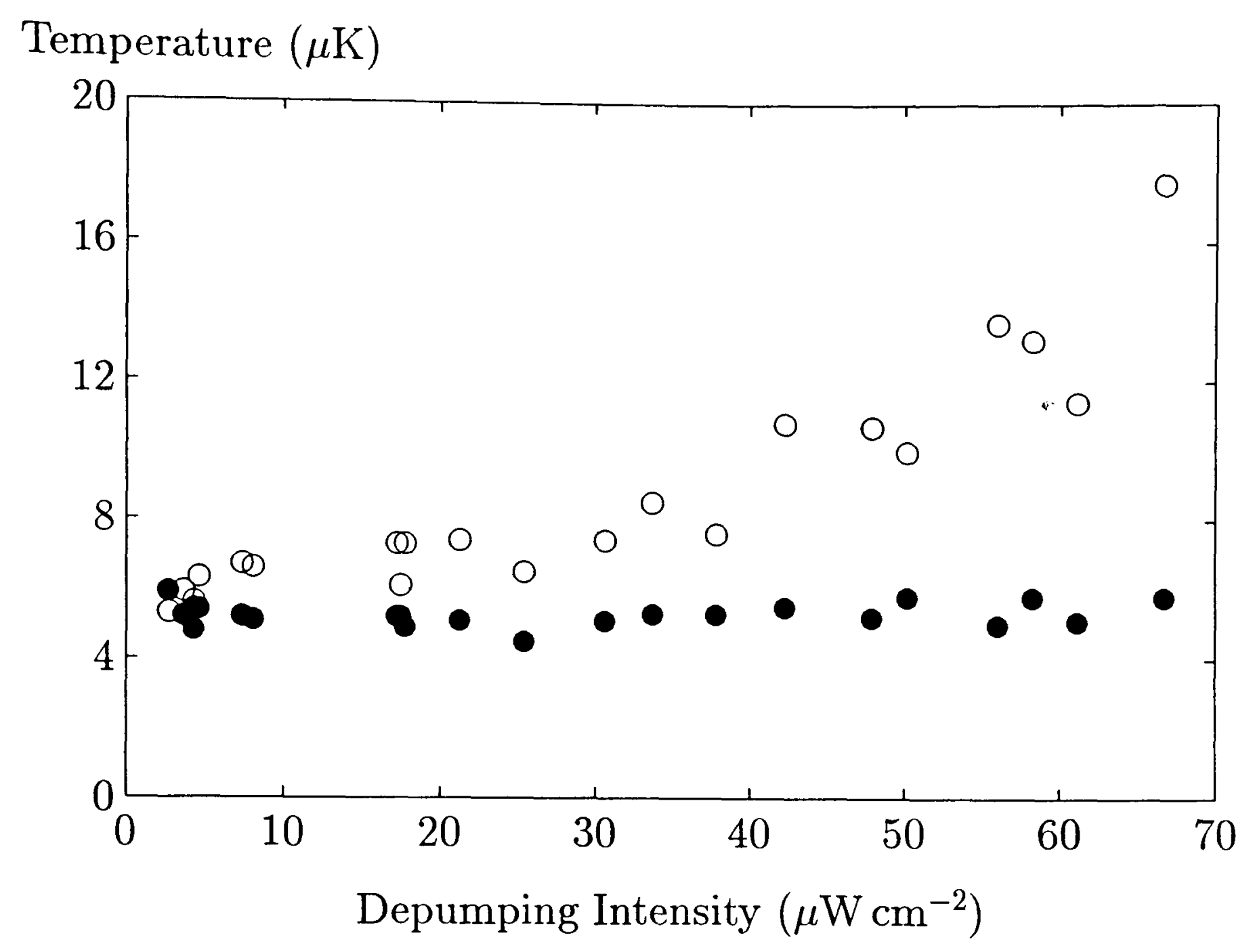

Figure 6.5: Molasses temperatures with (०) and without $(\bullet)$ depumping light.

temperatures with no depumping light and for these measurements single shots were sufficient. Their trend confirms that the behaviour of the system was constant during the measurements and their scatter indicates the minimum uncertainty of a single temperature measurement.

The breakdown in efficiency of sub-Doppler cooling is clear. According to a rate equation estimate similar to Eq. (6.9), an intensity of about $6 \mu \mathrm{Wcm}^{-2}$ of $F=4 \rightarrow 4^{\prime}$ depumping light is required to reduce the lifetime in the upper ground state to the molasses damping time of $40 \mu \mathrm{s}$ measured by Drewsen et al. [25]. This is consistent with the data shown here: at this intensity the temperature has increased significantly (by about $30 \%)$. At depumping intensities greater than about $50 \mu \mathrm{Wcm}^{-2}$ the TOF signal started to exhibit non-Gaussian wings, indicating a non-Gaussian velocity distribution. This is consistent with the idea of a breakdown in the cooling mechanism.

\subsubsection{Saturation of repumping}

In the MOT of a heavy alkali it is well-known empirically that the amount of light required to saturate the repumping transition for atoms in the trapped cloud is much less than that required to do the same for those atoms undergoing capture. This 
difference is a result of two factors. First, the intensity needed to achieve the former is very small in the heavy alkalis because of the small natural depumping rate. Secondly, an atom in the outer part of the trapping volume experiences various Doppler and Zeeman shifts which effectively increase the detuning of the repumping laser away from resonance. For example, for a typical capture velocity of $15 \mathrm{~ms}^{-1}$ the Doppler shift is more than three linewidths.

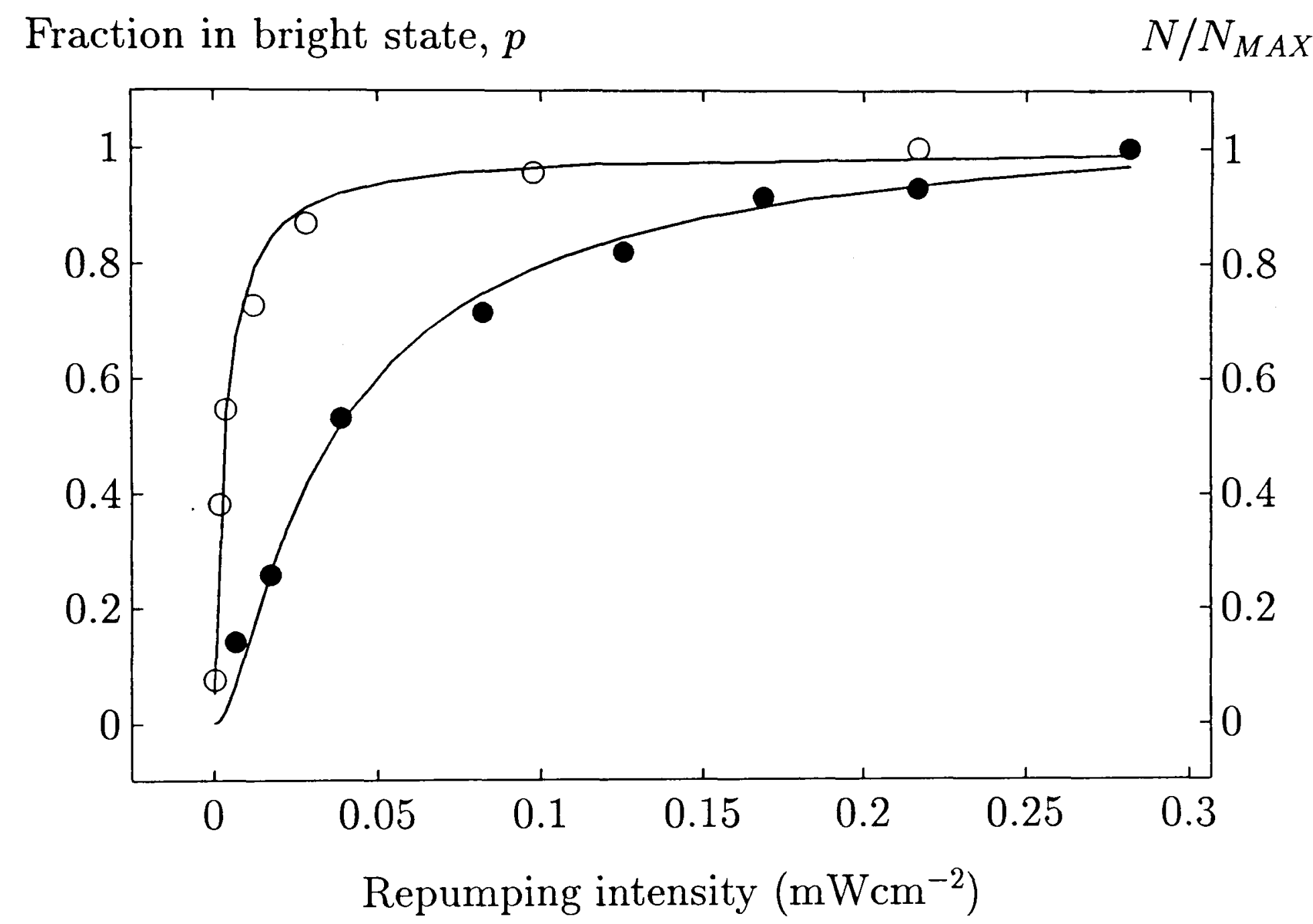

Figure 6.6: Saturation measurements with repumping laser: o for the cooling cycle ( $p$ scale), - for the loading cycle (number scale, $N$ ), together with fitted theoretical curves (see text).

A measurement of these two different saturation processes is shown in Fig. 6.6. The open circles are a measure of the repumping at the trap centre and were obtained by reducing the repumping intensity transiently in a fully-loaded MOT, with $p$ calculated from the ratio of fluorescence after the reduction to that before. The filled circles are measurements of the steady-state trap number as the repumping intensity was varied. The solid curve through the open circles is a least squares fit of the equation

$$
p=\frac{1}{1+\left[I_{t o t} \Gamma^{2} / 4 I_{r e p}(\Delta+\delta)^{2}\right]}
$$

with the total intensity $I_{\text {tot }}$ floated as a free parameter. Eq. (6.24) is a rate equation estimate of the variation expected and is derived from Eqs. (6.1) and (6.2), the rate 
equation (6.9) for the natural depumping rate from the intensity $I_{t o t}$, and a similar rate equation for the repumping rate from a repumping intensity $I_{\text {rep }}$. The curve through the filled circles is a product of this first fit and a factor accounting for the shift in detuning of the repumping light in the outer part of the trapping volume. The least squares fit for this second curve suggests that the average size of the shift is of the order of a linewidth at the edge of the trapping region [16].

As explained previously, it is precisely the spatial separation of repumping requirements which leads to the concept of the spatial dark MOT. Unfortunately this difference in requirements, as illustrated by Fig. 6.6, is so large as to make the realization of a significant population in the dark level impossible without the use of a forced depumping. That is, it was found that the small amount of stray light scattered from the outer trapping region into the centre was enough to keep $p$ very close to unity. A similar problem was found to occur with rubidium [2]. Slightly reduced values of $p$ were obtained by reducing the trapping beam intensity (to reduce the amount of stray scattering) or by detuning the repumping light away from resonance (to reduce the repumping rate), but to obtain large fractions it was found that depumping light was essential.

\subsubsection{Number and cross-section measurements}

The variation with $p$ of the number trapped in the spatial dark MOT for different scattering conditions was measured. A typical trend is shown in Fig. 6.7. The solid line is the least squares fit of Eq. (6.17) to the data points at $p<0.25$ with the total scaling and the product $\rho_{e e}\left(\sigma_{e} / \sigma_{g}-1\right)$ floated as free parameters. It can be seen that the equation gives a close match to the number variation at small values of $p$, but at higher values, where there is little number variation, the agreement is very poor. The larger numbers at small $p$ were observed to decay when the conditions were switched from dark MOT to MOT (in order to measure the number).

The observations described above were consistently observed at different scattering rates and on many different experimental runs. The increase in number permits a rough estimate of the ratio of excited to ground state cross-sections, $\sigma_{e} / \sigma_{g} . \rho_{e e}$ is estimated from a knowledge of total trapping intensity in the manner described in Sec. 4.3 and a ratio then follows by comparing the total increase in number with that predicted by Eq. (6.17). Because the variation in number around $p=1$ is not accurately reproduced by the formula, this procedure does not always yield the same value for $\sigma_{e} / \sigma_{g}$ as that obtained by the least squares fit to the variation in number at small $p$. For this reason it is estimated that the method described is only accurate to a factor $\sim 2$. The following trends were clearly observed. At high scattering rates that might be typically used for efficient loading of a trap $\left(\rho_{e e} \sim 0.3\right)$ the dark MOT number was seen to increase by a factor $\sim 2$ as $p$ was reduced. This is the small gain predicted in Sec. 6.2.4 and implies $\sigma_{e} / \sigma_{g}$ to be in the range $3.5-5.5$. At lower intensities this ratio increased, reaching the range $30-40$ after a reduction of intensity by a factor $\sim 3\left(\rho_{e e} \sim 0.15\right)$. The corresponding ratio of numbers was $N_{d M O T} / N_{M O T} \sim 5-7$. The qualitative conclusion of this behaviour is that $\sigma_{e} / \sigma_{g}$ varies more quickly than the $v_{e s c}^{-1 / 3}$ dependence predicted 


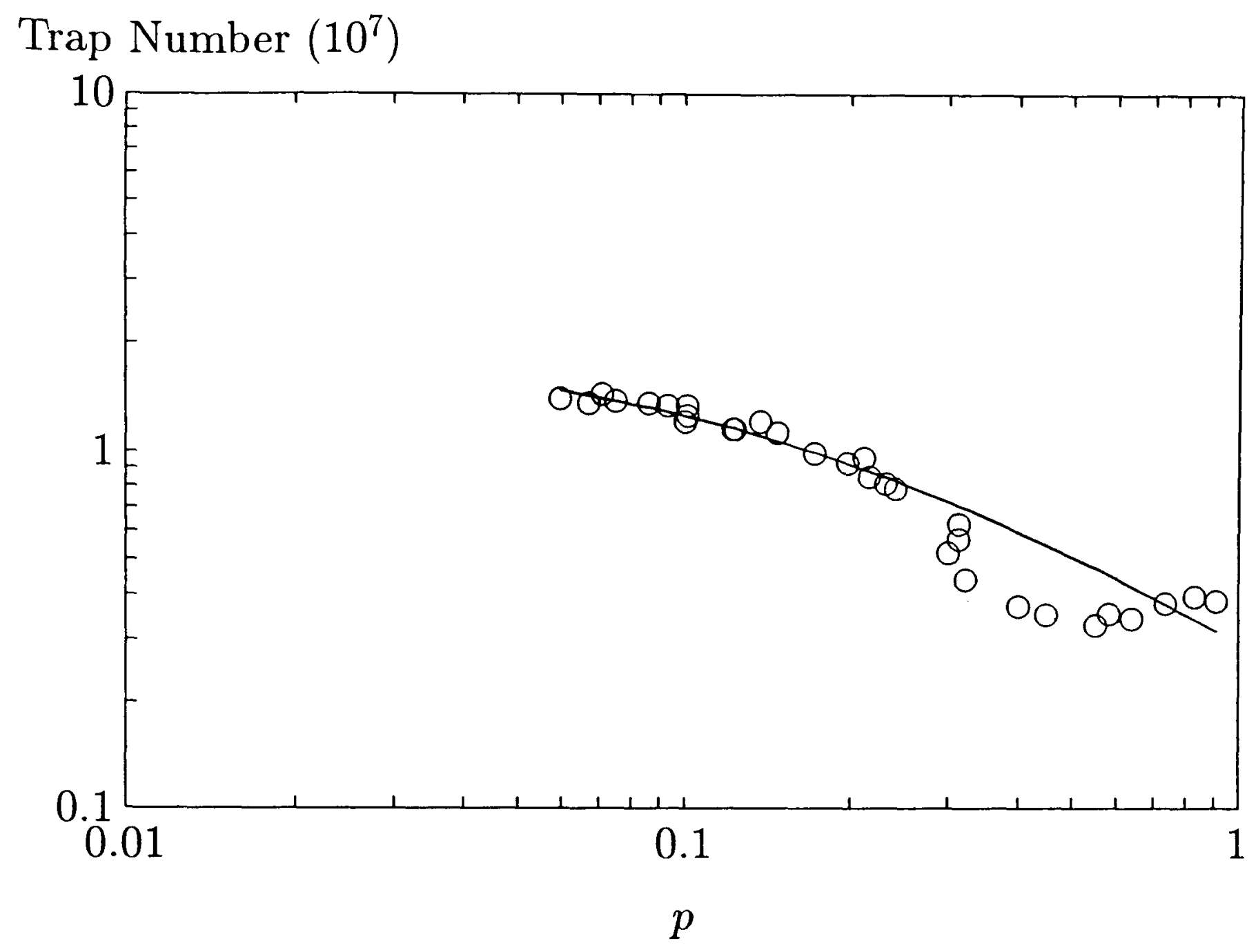

Figure 6.7: Number in the spatial dark MOT as a function of the dark SPOT parameter, $p$. The trapping parameters were $\delta=-2 \Gamma, \Omega=0.9 \Gamma$ and $\mathrm{d} B / \mathrm{d} z=15 \mathrm{Gcm}^{-1}$. The solid line is Eq. (6.17) with a best fit parameter $\sigma_{e} / \sigma_{g} \approx 35$ for the data points at $p<0.25$.

by Eq. (6.18). Further evidence of this is reported in the following section.

\subsubsection{Density measurements}

The absolute value of the densities reported in this section can be compared directly with those quoted in chapter 4 . Other considerations discussed in that chapter concerning the determination of the density also apply. In particular, it was again necessary to make use of two orthogonally-positioned cameras for obtaining trap images; in general for $p \gtrsim 0.3$ measurements of all three independent dimensions were necessary to accurately determine the trap volume. For smaller dark state fractions it was sufficiently accurate (to better than $10 \%$ ) to use the two dimensions obtained from a single camera, but the use of two cameras with different apertures permitted the dynamic range of detection to extend down into the regime $p \lesssim 0.05$. It is estimated that each density measurement is only accurate to $50 \%$ at the absolute level, mainly because of the uncertainty in estimating the trapped number from the emitted fluorescence, whilst measurements within a data set can generally be compared at the $10-20 \%$ 
level. In chapter 4 a best MOT density of about $1.5 \times 10^{11} \mathrm{~cm}^{-3}$ was reported. This was obtained under conditions of high magnetic field $\left(40 \mathrm{Gcm}^{-1}\right)$ and a reduced light shift parameter $\left(\Omega^{2} / \delta \Gamma \lesssim 0.3\right)$. This density is roughly a factor 15 higher than that which was measured under the standard loading conditions, of which roughly a factor 5 was due to the increased gradient with the reduced scattering rate accounting for the remaining factor of 3 .

\subsubsection{Temporal densities}

The dark MOT is most easily realized in caesium by a transient reduction of the repumping intensity. In the original sodium work [51] it was reported that this temporal formation of a dark MOT produced compressions almost as large as those in the spatial configuration. A temporal dark MOT in caesium was formed with the following procedure. The MOT was fully loaded to roughly $10^{7}$ atoms at a detuning $\delta=2 \Gamma$. The trapping intensity was then reduced to decrease the light shift parameter to $\Omega^{2} / \delta \Gamma \approx 0.5$, and simultaneously the magnetic field gradient was increased from 10 to $40 \mathrm{Gcm}^{-1}$. The combined effect of this was to increase the MOT density by roughly a factor 4 to about $5 \times 10^{10} \mathrm{~cm}^{-3}$. After a wait of $100 \mathrm{~ms}$ the repumping intensity was then reduced and locked to the desired level, and the subsequent trap decay was then monitored in an identical manner to that described in chapter 4. No depumping light was used.

Crucially, compression in the temporal dark MOT was observed only when the scattering rate of the trapping photons was high. The rate was found to be high enough when the total saturation parameter $s_{\text {tot }}$ defined by

$$
s_{t o t}=\frac{\Omega_{t o t}^{2} / 2}{\Gamma^{2} / 4+\delta^{2}}
$$

was greater than about 0.2 . This requirement of a high scattering rate is equivalent to specifying that the trap must be well into the multiple scattering regime [83]. When the scattering rate was sufficiently high, large compressions were observed. As $p$ was reduced from unity a roughly linear increase in density was observed, as expected in the multiple scattering regime [Eq. (6.4)], and at $p \approx 0.15$ the density had increased to somewhere between 3 and $4 \times 10^{11} \mathrm{~cm}^{-3}$, an improvement by a factor of $2-3$ over the best density that has been attainable in our MOT. An example of this increased compression is shown in Fig. 6.8. As $p$ was reduced the trap lifetime was observed to decrease rapidly. For $p \lesssim 0.2$ it was comparable to the $20 \mathrm{~ms}$ resolution of the detection system and for reasons discussed below this made it extremely difficult to measure precisely the density. It was thus not possible to deduce information about the maximum density attainable with this technique nor the density variation in the temperature-limited and free travel-limited regimes.

When $p$ was reduced below about 0.3 (for the trapping conditions described above) it was noticed that the density profile began to exhibit a two-component structure. A dense central Gaussian distribution was surrounded by a much more diffuse Gaussian extending out to large radius. Typically the fraction of atoms in the central component was in the range $0.5-0.7$ for $0.04 \lesssim p \lesssim 0.2$ and the dark MOT densities quoted in 


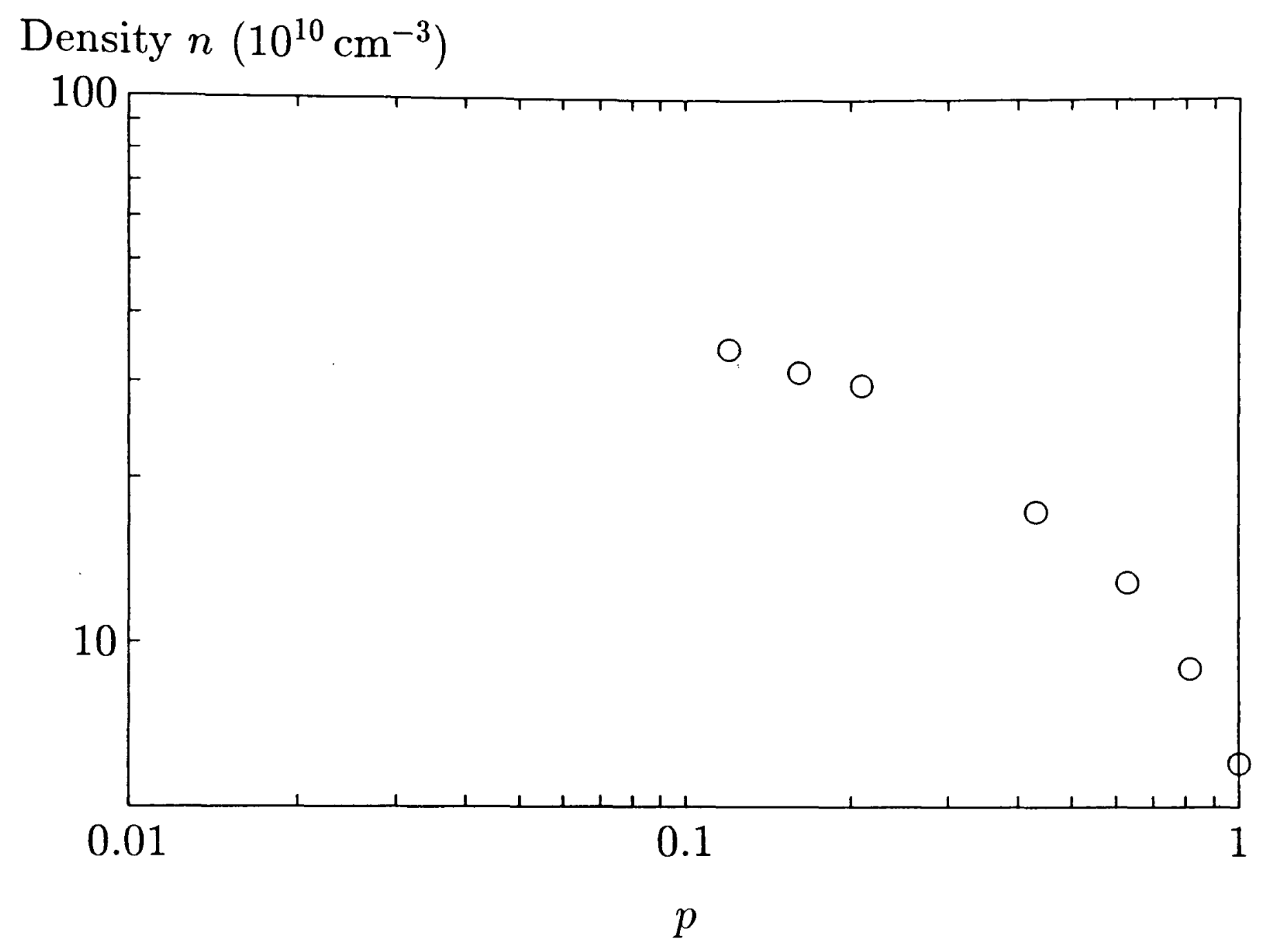

Figure 6.8: Density in the temporal dark MOT as a function of the dark SPOT parameter. The measurements are not accurate for $p \lesssim 0.2$.

this section are those of this central region. The shape was identical in appearance to that profile described in chapter 4 when in the MOT at low light shifts or high field gradients, the spatial breakdown of the spring constant was observed to occur within the trapped cloud of atoms. However, the dimensions of the dense central region in the dark MOT were measured to be roughly a factor $p$ smaller than those in the equivalent MOT, a fact which is not explainable by the simple condition Zeeman shift $\sim$ light shift [Eq. 2.19]. Nevertheless, we believe that the profile was not caused by free travel in the dark level for the following reasons. First, the same two-component shapes were observed at similar values of $p$ in the spatial dark MOT with strong depumping. With such depumping the free travel in the dark state is much reduced. Secondly, the dimension of the underlying profile decreased (slowly) as the repumping intensity was reduced, an effect which is opposite to the variation one would expect from free travel.

The reduction in trap lifetime as $p$ was reduced was rapid. For $p \lesssim 0.15$ the lifetime was observed to be shorter than the $20 \mathrm{~ms}$ integration time of a standard video signal. Under these circumstances the standard signal is useless for determining the trap density because the decay of the trap dimensions weights the integrated profile near the trap centre in an undetermined way. Moreover, lifetimes this short are comparable 
to the time it takes for the density to compress (roughly one third of the position damping time) and are then themselves a density-limiting factor (Sec. 2.3). The spatial dark MOT is a very straightforward method for investigating densities at large dark state fractions and for this reason it was not attempted to use the temporal method (with, for example, a faster detection scheme) to study in any detail the temperaturelimited and free travel-limited density regimes.

It is interesting to consider the origin of the short lifetimes. By comparing the decay rates of the dense central region and its diffuse surround, it was possible to estimate the relative contributions of intra-trap cold collision losses and background vapourtrapped atom collisions to the decay of the number in the central region. At $p \sim 0.2$ it was found that the contributions were similar (the vapour background pressure was $\sim 10^{-8}$ Torr as usual).

The significance of cold collision processes in the dark MOT can be shown by the following argument. In the MOT the collision events that are important involve each participating atom to be (at least initially) in the cooling cycle. Their collision crosssections are thus reduced by the factor $p^{2}$ in the dark MOT and consequently the trap loss rate they cause is reduced by at least the factor $p$ (since the dark MOT density only increases in multiple scattering regime, and then only as $p^{-1}$ ). Therefore, for small $p$ these processes may be considered to be insignificant in the dark MOT. However, exactly analogous events, involving one of the colliding atoms initially in the dark level, are relevant in the dark MOT because the cross-sections for these events scale as $p(1-p)$. Thus in the multiple scattering regime, where the density increases as $p$, their loss rate is only reduced from the similar process in the MOT by the factor $(1-p)$. We thus conclude that, at least in the multiple scattering regime, the dark MOT and MOT should have similar loss contributions from cold collision. This argument allows us to identify the cold collision event contributing to the very rapid decays described above. Accurate measurements of cold collision rates in a caesium MOT under different trapping conditions have been made (chapter 5), and decay times as short as those seen in the temporal dark MOT $(<50 \mathrm{~ms})$ have only been observed when the MOT has been sufficiently weak for hyperfine-changing collisions to make a contribution to trap loss. The hyperfine-changing event which is significant in the dark MOT is

$$
\mathrm{Cs}(F=3)+\operatorname{Cs}(F=4) \rightarrow \operatorname{Cs}(F=3)+\operatorname{Cs}(F=3)
$$

and it is therefore suggested that this is the process predominantly responsible for the cold collision component of the rapid decays. An estimate of the cold collision rate coefficient $\beta$ (defined by Eq. 2.33) from the cold collision contribution to the decay is $8 \times 10^{-11} \mathrm{~cm}^{3} \mathrm{~s}^{-1}$ at $p \approx 0.2$. This is very similar to the caesium MOT hyperfine loss rate measured by Sesko et al. [74].

The observation of a lifetime $<50 \mathrm{~ms}$ against background collisions when $p<0.2$ is also not surprising. For this reduced repumping intensity we have just suggested that the trap depth is small enough to be overcome by the kinetic energy released in a hyperfine-changing collision. Similarly short lifetimes have been observed in the caesium MOT when the trapping has been sufficiently weak (chapter 5). Furthermore, these rapid decay rates in the MOT are observed to increase significantly as the trap 
depth is reduced. A similar observation was made by Wallace et al. [87]. It is interesting to compare these observations with the classical estimate of the collision rate with the background vapour made by Steane et al. [77] discussed in detail in Sec. 2.4.1 [and from which Eq. (6.18) originates]. According to that calculation the background collision rate should increase only slowly with decreasing trap escape velocity $\left(\sigma_{g} \propto v_{e s c}^{-1 / 3}\right)$. This is at odds with the experimental evidence just presented and is further support for the remark made at the end of Sec. 6.4.3, that the ability of a weak trap to retain atoms with small velocities is worse than a classical calculation would suggest. The failure of a classical description was discussed in the previous chapter.

\subsubsection{Steady-state densities}

The spatial dark MOT permits a more comprehensive investigation of the density regimes described in Sec. 6.2.2. In particular it allows easy access to the dark state fractions for $p<0.2$.

The following general observations were made of the behaviour of the dark MOT. The density in the multiple scattering regime behaves in a similar manner to that of the temporal dark MOT. There is no compression if the scattering rate is too low or if the trapped number is too small $\left(N \lesssim 10^{6}\right)$, the latter being in agreement with the predictions of the model presented. As $p$ is reduced a two-component density profile is eventually observed, although it is generally difficult to discern unless the density in the central region is in excess of $2 \times 10^{11} \mathrm{~cm}^{-3}$. When the conditions are right for compression, the density is seen to increase in good accordance with Eq. (6.4). The density rises by about an order of magnitude after which the trap enters the temperature-limited regime, and further reduction of $p$ then causes the density to decrease.

For the reason illustrated by Figs. 6.2(b) and 6.2(c) the steady-state dark MOT was operated under conditions optimal for loading a large number of atoms. The field gradient used was necessarily small $\left(15 \mathrm{Gcm}^{-1}\right)$ and consequently the maximum density reached was just over $10^{11} \mathrm{~cm}^{-3}$. This is a similar improvement in the density to that achieved in the MOT by transiently reducing the scattering rate and increasing the field gradient to $40 \mathrm{Gcm}^{-1}$. For two reasons it is desirable to be able to use the spatial dark MOT with a larger field gradient. First, the measurements in chapter 4 show that this would allow the dark MOT compression to start from a higher initial density, i.e. at a given dark state fraction the dark MOT density is then higher. Secondly, an increased field gradient increases the trap spring constant thereby reducing the temperature-limited volume. The temperature-limiting effect then sets-in at a higher density. However, as remarked in Sec. 6.2.2, the capture efficiency of a MOT decreases sharply with increasing field gradient. For example, at $40 \mathrm{Gcm}^{-1}$ over an order of magnitude fewer atoms were loaded than at $15 \mathrm{Gcm}^{-1}$ and no compression was observed in the dark MOT. This problem was circumvented, however, by the use of both spatial and temporal trapping. A large number of atoms was loaded into the spatial configuration using the field gradient of $15 \mathrm{Gcm}^{-1}$ and the gradient was then increased transiently to produce extra compression. Because the trap depth is determined by the region of high repumping surrounding the trap, this transient procedure 
did not invoke the very rapid decays seen in the temporal dark MOT.

A density variation with $p$ using this technique is shown in Fig. 6.9 for around $10^{7}$ atoms. It should be noticed that the starting $(p=1)$ density is indeed high. The solid line is the linear compression expected in the multiple scattering regime [Eq. (6.4)] and the dotted line is the fall-off predicted in the temperature-limited regime by Eq. (6.7). The molasses temperature for the latter has been set to approximately $105 \mu \mathrm{K}$, which is about a factor $\sim 2$ larger than would expected from the measurements of Sec. 4.4 for the trapping conditions used. This discrepancy is most likely to be the result of the forced depumping. The maximum density of just under $10^{12} \mathrm{~cm}^{-3}$ was the largest observed in the dark MOT and is a factor $\sim 5$ larger than the largest compression obtained in the MOT.

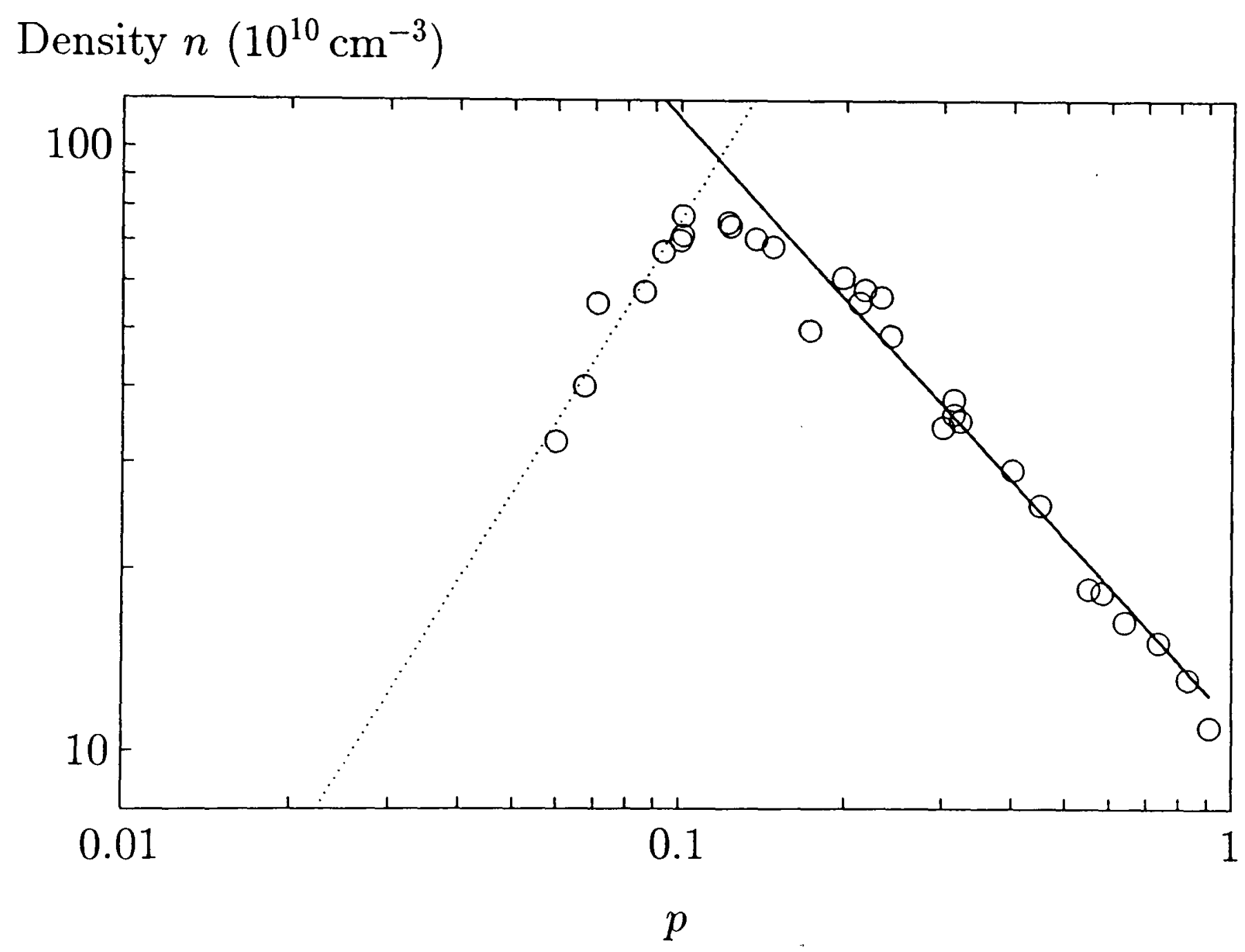

Figure 6.9: Density in the spatial dark MOT as a function of the dark SPOT parameter, $p$. The loading parameters $\delta=2 \Gamma, \Omega=0.9 \Gamma$ and $\mathrm{d} B / \mathrm{d} z=15 \mathrm{Gcm}^{-1}$ were used to trap between $4 \times 10^{6}$ and $1.4 \times 10^{7}$ atoms (depending on $p$ as shown in Fig. 6.7) and the field gradient was then transiently increased to $40 \mathrm{Gcm}^{-1}$ to produce the densities shown. The solid line is the density variation in the multiple-scattering regime as predicted by Eq. (6.4), and the dotted line is that of Eq. (6.7) for the temperature-limited regime, with a molasses temperature of $105 \mu \mathrm{K}$.

The data in Fig. 6.9 present the picture of a dark MOT density increasing in the multiple scattering regime and subsequently decreasing in the temperature-limited regime. Two checks were performed to confirm that the maximum densities were indeed 
temperature-limited. First, the trapping intensity was increased at the maximum density. The density was observed to decrease, consistent with the idea of increasing the temperature-limited volume by heating the atoms (albeit with the temperature modified by the factor $\sim 2$ from the forced depumping). Secondly, it was verified that the densities were not free-travel limited. To do this the beam expansion of the depumping beam was reduced to increase the intensity of the $F=4 \rightarrow 4^{\prime}$ light at the trap centre to about $2 \mathrm{mWcm}^{-2}$. By varying the detuning of this depumping light and the intensity of the repumping fill-in beam, the dark state fraction was kept constant at that value giving the highest density $(p \sim 0.1)$ whilst the relative amounts of depumping and repumping, and thus the distance travelled in the dark level, was varied. No increase in the maximum density was observed as the depumping rate was increased from the minimum value required to reach $p \sim 0.1$ in the spatial configuration, confirming the initial hypothesis. It should be appreciated, however, that the minimum amount of depumping light needed to lower $p$ to $\sim 0.1$ in the spatial scheme is itself large enough to reduce substantially the lifetime in the upper ground state, and consequently the free travel distance for a given $p$. However, by comparing the $8 \times 10^{11} \mathrm{~cm}^{-3}$ maximum density in Fig. 6.9 with the density $\gtrsim 4 \times 10^{11} \mathrm{~cm}^{-3}$ attained in the temporal dark MOT with very similar trapping conditions (as explained in the previous section, it was not possible to measure the maximum temporal density precisely), one can conclude that the worst possible effect of free travel for the conditions used (i.e. when $p \sim 0.1$ is attained by reducing the repumping intensity only) is to reduce the maximum density by some factor of less than two. A reduction by this amount is consistent with the prediction by the dark MOT model using the earlier estimate of $250 \mu$ s for the lifetime in the upper ground state against natural depumping.

The value of increasing the field gradient to improve the maximum density has been demonstrated. Unfortunately the setup used in this work was only able to generate gradients up to $45 \mathrm{Gcm}^{-1}$. The alternative methods to improve upon the maximum density in Fig. 6.9 were investigated and are now discussed. In principle, the density in the temperature-limited dark MOT is increased both by loading more atoms and reducing the trap volume by cooling the cloud. However, it should be appreciated that in caesium it is not immediately obvious that either of these steps should lead to a significant improvement over that of Fig. 6.9. A higher density will occur at a smaller value of $p$ and will therefore require stronger depumping for its observation; in turn this will generate additional heating which will tend to increase the temperature-limited volume and thus reduce the density.

It was not possible to properly test the effect of loading more atoms on the density. With a total beam intensity of around $40 \mathrm{mWcm}^{-2}$ available for trapping and with the trap detuning and field gradient near optimal for efficient loading, typically $10^{7}$ atoms were loaded into the MOT. This could be increased to close to $5 \times 10^{7}$ atoms but only by increasing the caesium vapour pressure substantially above its standard operating value $\left(\sim 10^{-8}\right.$ Torr $)$. The increased pressure had to be very close to that value at which collisions with the background vapour prevent atoms from being captured, and the intensity imbalance between a trapping beam and its retroreflection was correspondingly very large (estimated at around $40 \%$ ). Under these conditions the trap 
was very unstable and its shape highly asymmetrical. It was consequently impossible to make meaningful measurements of the density.

The effect of attempting to cool the cloud was investigated in the following way. Under the conditions of Fig. 6.9 a near-maximum density $(p \sim 0.15)$ was measured for decreasing trapping intensities. At first the density remained roughly constant, as expected ${ }^{3}$. However, when the light shift parameter $\Omega^{2} / \delta \Gamma$ was reduced below about 0.2 the density significantly decreased. This may be interpreted as the same breakdown in spatial confinement at low light shifts that was observed in the MOT, and it implies that no gains in density are to be made by cooling with a light shift parameter of less than about 0.2. According to Fig. 4.3 the molasses temperature at $\Omega^{2} / \delta \Gamma=0.2$ and at $\delta=2 \Gamma$ is about $30 \mu \mathrm{K}$, and we know that in the dark MOT the heating effect of the forced depumping will double this temperature. Now according to the model of the dark MOT the maximum density reached at this colder temperature of $\sim 60 \mu \mathrm{K}$ is just over $10^{12} \mathrm{~cm}^{-3}$, i.e. no significant increase over the density at $\sim 100 \mu \mathrm{K}$. It therefore seems reasonable to conclude that the maximum dark MOT densities that were observed were very close to the maximum achievable with the trapping apparatus available.

\subsection{Concluding summary}

The work presented in this chapter has demonstrated that the MOT can be applied successfully to a heavy alkali to produce large improvements in density, and this should pave the way for evaporative cooling of caesium atoms. The conditions that have been used to reach densities of nearly $10^{12} \mathrm{~cm}^{-3}$ are considerably different to those of the original sodium experiment. In that work an atomic beam and Zeeman slower apparatus were used to trap more than $10^{10}$ atoms, and densities approaching $10^{12} \mathrm{~cm}^{-3}$ were reached by confining $99 \%$ of the atoms to the dark level at low field gradients $\left(10-15 \mathrm{Gcm}^{-1}\right)$. No forced depumping was necessary because the natural off-resonant excitation rate into the dark level was high. We have trapped in a standard vapour cell arrangement, and have compensated for the relatively small trapping number $\left(\sim 10^{7}\right.$ atoms) with an increased field gradient $\left(40 \mathrm{Gcm}^{-1}\right)$, applied transiently after loading. In the spatial dark MOT configuration maximum densities were observed with $90 \%$ of the atoms in the dark level and to obtain this dark ștate fraction depumping atoms out of the cooling cycle was essential.

It has been found that the density behaviour of the spatial dark MOT is adequately predicted by a simple model whose main assumption is that the trap spring constant is reduced by the fraction of time each atom spends in the cooling cycle. The process limiting the maximum density has been shown to be the temperature-limited volume. In turn, the temperature determining this volume is dominated by the heating effect of forced depumping. With a combination of spatial and transient trapping densities of almost $10^{12} \mathrm{~cm}^{-3}$ were observed, an improvement by a factor $\sim 5$ over the best

\footnotetext{
${ }^{3}$ More precisely there was a slow trend in the density which was probably the small variation of the $p=1$ (MOT) density with trapping intensity.
} 
attained MOT density, and an improvement by almost an order of magnitude over the MOT density under identical trapping conditions. It should be mentioned once again that this improved density in caesium is still a factor 3 lower than Drewsen et al. [25] reported in an ordinary caesium MOT. We have suggested that free travel in the dark level is not a significant density-limiting effect and have given evidence to support this.

The heating effect of optically pumping atoms out of the cooling cycle has been demonstrated directly. The depumping necessary to reach the highest densities in a caesium spatial dark MOT induces increased trap temperatures by some factor $\sim 2-3$. Consequently, the spatial dark MOT is not a technique in itself for attaining high phase-space densities.

It has been shown that the dark MOT can yield high densities if the dark state fraction is increased transiently. However, in this temporal scheme large background vapour-trapped atom and intra-trap cold collision loss rates make the trapping lifetimes exceedingly short and render the scheme impractical. Moreover, for dark level populations of $80 \%$ or more the lifetime is reduced to a few tens of milliseconds or less, which is comparable to the position damping time of an atom in the trap. In this instance the decay itself is a density-limiting mechanism. 


\section{Chapter 7}

\section{Concluding remarks}

The aim of the experimental and theoretical work described in this thesis was to understand the processes limiting the phase-space density of caesium atoms in a magnetooptical trap. The knowledge gained is summarized as follows. The study of the conventional MOT indicated that $\rho$ can be increased by over two orders of magnitude to around $10^{-5}$ by a combination of transiently increasing the magnetic field gradient and simultaneously reducing light shift. In such a "compressed" MOT the density is limited by reabsorption of photons scattered within the cloud, and not by cold collisions. At small values of the light shift parameter there is a reduction in the spatial density of atoms and this prevents the maximum density being combined with the minimum temperature. Consequently $\rho$ is a factor $\sim 3$ smaller than one might otherwise hope for. The investigation of the dark MOT revealed that the spatial density of caesium atoms can be increased by roughly an order of magnitude but at a cost of considerable heating due to optical pumping into the dark level. The phase-space density is consequently lower in the dark MOT than in the conventional MOT. It seems possible, however, that one could combine the high density of the dark MOT with the lowest temperature attainable in the MOT by using a transient approach similar to that suggested at the end of chapter 4 . A spatial density of $10^{12} \mathrm{~cm}^{-3}$ at a temperature of $3 \mu \mathrm{K}$ implies $\rho \approx 6.5 \times 10^{-4}$, and as a starting condition for loading into a magnetic trap and subsequent evaporative cooling this compares extremely favourably with recent experiments observing $\mathrm{BEC}$ in atomic alkalis. For example, in the work with rubidium [1] atoms loaded into a magnetic trap were first cooled and compressed, but only to $20 \mu \mathrm{K}$ and about $10^{11} \mathrm{~cm}^{-3}$ respectively. In fact, this value for the density is not directly given in [1] but it is possible to make an estimate from other numbers given. In the next few months there will be a working time-orbiting potential (TOP) trap in Oxford for caesium atoms, similar to the one used for rubidium, and it will then be possible to compare numbers more directly.

This work has attempted to understand the processes limiting the density itself. An important observation was that the density in the MOT does not increase rapidly with increased laser detuning and decreased intensity, the simplest explanation for which is that the theory proposed by Walker, Sesko and Wieman does not account accurately for the delicate balance between attractive and repulsive forces. The observation of a much higher density in the dark MOT than in the conventional MOT indicates that 
the repulsion effect of multiple scattering is best supressed by reducing the time spent in the cooling cycle rather than reducing the scattering rate itself. It is interesting to consider that it further suggests that the sensitivity of the MOT density to trapping beam quality is unlikely to be responsible for the large discrepancy in the densities measured in this work and those quoted by Drewsen et al. [25].

Aside from considerations of spatial densities and phase-space, this thesis has contributed in other ways to the understanding of the caesium MOT. Observations of the atomic density and velocity distributions in a cloud extending beyond the capture region of the confining force were reported for the first time. Uncertainties in the spring constant and of the temperature in optical molasses have been reduced, and evidence for some degree of spatial confinement in the three-dimensional light field of the MOT has been given. Effects of cold collisions have been measured and used to assess their effect on the dynamics of the compressed MOT, and collision rates with the background vapour for shallow traps have been studied in several different ways. Finally, a simple model predicting the behaviour of the dark MOT has been described and its main features justified by measurement of temperature, number and density. 


\section{Bibliography}

[1] M. H. Anderson, J. R. Ensher, M. R. Matthews, C. E. Wieman, and E. A. Cornell. Observation of Bose-Einstein condensation in a dilute atomic vapor. Science, 269:198-201, 1995.

[2] M. H. Anderson, W. Petrich, J. R. Ensher, and E. A. Cornell. Reduction of lightassisted collisional loss rate from a low-pressure vapor-cell trap. Phys. Rev. A, 50(5):R3597-R3600, 1994.

[3] Roger W. Anderson. Quantum correction factors for classical small angle scattering by inverse power potentials. J. Chem. Phys., 60(7):2680-2683, 1973.

[4] V. S. Bagnato, L. C. Marcassa, M. Oria, G. I. Surdutovich, R. Vitlina, and S. C. Zilo. Spatial distribution of atoms in a magneto-optical trap. Phys. Rev. A, 48(5):3771-3775, 1993.

[5] Y. B. Band and P. S. Julienne. Optical-Bloch-equation method for cold-atom collisions: Cs loss from optical traps. Phys. Rev. A, 46(1):330-343, 1992.

[6] H. J. M. J. Boesten, B. J. Verhaar, and E. Tiesinga. Quantum suppression of collisional loss rates in optical traps. Phys. Rev. A, 48(2):1428-1433, 1993.

[7] C. C. Bradley, C. A. Sackett, J. J. Tollett, and R. G. Hulet. Evidence of BoseEinstein condensation in an atomic gas with attractive interactions. Phys. Rev. Lett., 75(9):1687-1690, 1995.

[8] K. Burnett. Private communication.

[9] Y. Castin. Private communication.

[10] Y. Castin, K. Berg-Sørensen, K. Mølmer, and J. Dalibard. In F. Ethlothzky, editor, Fundamentals of Quantum Optics III. Springer-Verlag, Berlin, 1993.

[11] Y. Castin and J. Dalibard. Quantization of atomic motion in optical molasses. Europhys. Lett., 14(8):761-766, 1991.

[12] Y. Castin and K. Mølmer. Monte Carlo wave-function analysis of 3D optical molasses. Phys. Rev. Lett., 74(19):3772-3775, 1995.

[13] M. A. M. Chowdhury. Laser cooling of caesium atoms applied to time standards. D.Phil. thesis, Oxford University, 1993. Unpublished. 
[14] C. Cohen-Tannoudji. Atomic motion in laser light. In Fundamental Systems in Quantum Optics, Les Houches, pages 703-716, Amsterdam, 1992. North-Holland.

[15] C. Cohen-Tannoudji, J. Dupont-Roc, and G. Grynberg. Atom-Photon Interactions. Wiley, New York, 1992.

[16] C. J. Cooper. Laser cooling and trapping of atoms. D.Phil. thesis, Oxford University, 1995. Unpublished.

[17] C. J. Cooper, G. Hillenbrand, J. Rink, C. G. Townsend, K. P. Zetie, and C. J. Foot. The temperature of atoms in a magneto-optical trap. Europhys. Lett., 28(6):397-402, 1994.

[18] J. Dalibard. Helsinki workshop on laser manipulation of atoms, unpublished.

[19] J. Dalibard. Laser cooling of an optically thick gas: the simplest radiation pressure trap? Opt. Comm., 68(3):203-208, 1988.

[20] J. Dalibard and C. Cohen-Tannoudji. Laser cooling below the Doppler limit by polarization gradients: simple theoretical models. J. Opt. Soc. Am. B, 6(11):2023$2045,1989$.

[21] Jean Dalibard, Yvan Castin, and Klaus Mølmer. Wave-function approach to dissipative processes in quantum optics. Phys. Rev. Lett., 68(5):580-583, 1992.

[22] K. B. Davis, M. O. Mewes, M. R. Andrews, N. J. van Druten, D. S. Durfee, D. M. Kurn, and W. Ketterle. Bose-Einstein condensation in a gas of sodium atoms. Phys. Rev. Lett., 75(22):3969-3973, 1995.

[23] Kendall B. Davis, Marc-Oliver Mewes, Michael A. Joffe, Michael R. Andrews, and Wolfgang Ketterle. Evaporative cooling of sodium atoms. Phys. Rev. Lett., 74(26):5202-5205, 1995.

[24] M. T. de Araujo, L. G. Marcassa, S. C. Zillo, and V. S. Bagnato. Double ring structure: Another variant in the spatial distribution of cold sodium atoms. Phys. Rev. A, 51(5):4286-4288, 1995.

[25] M. Drewsen, Ph. Laurent, A. Nadir, G. Santarelli, A. Clairon, Y. Castin, D. Grison, and C. Salomon. Investigation of sub-Doppler cooling effects in a cesium magneto-optical trap. App. Phys. B, 59:283-298, 1994.

[26] O. Dulieu, J. Weiner, and P. Julienne. Accuracy of molecular data in the understanding of cold collisions. Phys. Rev. A, 49(1):607-610, 1994.

[27] K. Ellinger, J. Cooper, and P. Zoller. Light-pressure force in $N$-atom systems. Phys. Rev. A, 49(5):3909-3933, 1994.

[28] C. J. Foot. Laser cooling below the Doppler limit in a magneto-optical trap. In Proceedings of 'Enrico Fermi' Summer School on Laser Manipulation of Atoms and Ions, Varenna, Italy, pages 703-716, Amsterdam, 1992. North-Holland. 
[29] C. J. Foot, H. Wu, E. Arimondo, and G. Morigi. Optical elements for interferometry of atoms with $J>1$. J. Physique II, 4(11):1913-1924, 1994.

[30] A. Gallagher and D. E. Pritchard. Exoergic collisions of cold Na*-Na. Phys. Rev. Lett., 63(9):957-960, 1989.

[31] G. Gerz, T. W. Hodapp, P. Jessen, K. M. Jones, W. D. Phillips, C. I. Westbrook, and K. Mølmer. The temperature of optical molasses for two different atomic angular momenta. Europhys. Lett., 21(6):661-666, 1993.

[32] K. E. Gibble and S. Chu. Laser-cooled Cs frequency standard and a measurement of the frequency shift due to ultracold collisions. Phys. Rev. Lett., 70(12):1771$1774,1993$.

[33] K. E. Gibble, S. Kasapi, and S. Chu. Improved magneto-optic trapping in a vapor cell. Opt. Lett., 17(7):526-528, 1992.

[34] Kurt Gibble, Seongsik Chang, and Ronald Legere. Direct observation of $s$-wave atomic collisions. Phys. Rev. Lett., 75(14):2666-2669, 1995.

[35] P. L. Gould, P. D. Lett, P. S. Julienne, and W. D. Phillips. Observation of associative ionization of ultracold laser-trapped sodium atoms. Phys. Rev. Lett., 60(9):788-791, 1988.

[36] I. Guedes, M. T. de Araujo, D. M. B. P. Milori, G. I. Surdutovich, V. S. Bagnato, and S. C. Zilo. Forces acting on magneto-optically trapped atoms. J. Opt. Soc. Am. B, 11(10):1935-1940, 1994.

[37] T. W. Hänsch and A. Schawlow. Cooling of gases by laser radiation. Opt. Comm., 13(1):68-69, 1975.

[38] A. Hemmerich and T. W. Hänsch. Radiation pressure vortices in two crossed standing waves. Phys. Rev. Lett., 68(10):1492-1495, 1992.

[39] G. Hillenbrand, K. Burnett, and C. Foot. to appear in Phys. Rev. A.

[40] G. Hillenbrand, C. J. Foot, and K. Burnett. Heating due to long-range photon exchange interactions between cold atoms. Phys. Rev. A, 50(2):1479-1489, 1994.

[41] D. Hoffmann, P. Feng, and T. Walker. Measurements of Rb trap-loss collision spectra. J. Opt. Soc. Am. B, 11(5):712-720, 1993.

[42] D. Hoffmann, P. Feng, R. S. Williamson, and T. Walker. Excited-state collisions of trapped ${ }^{85} \mathrm{Rb}$ atoms. Phys. Rev. Lett., 69(5):753-756, 1992.

[43] M. J. Holland, K. A. Suominen, and K. Burnett. Cold collisions in a laser field: Quantum Monte Carlo treatment of radiative heating. Phys. Rev. A, 50(2):1513$1530,1994$. 
[44] M. J. Holland, K. A. Suominen, and K. Burnett. Quantal treatment of cold collisions in a laser field. Phys. Rev. Lett., 72(15):2367-2369, 1994.

[45] A. Höpe, D. Haubrich, G. Müller, W. G. Kaenders, and D. Meschede. Neutral cesium atoms in strong magnetic-quadrupole fields at sub-Doppler temperatures. Europhys. Lett., 22(9):669-674, 1993.

[46] K. Huang. Statistical Mechanics. John Wiley and Sons, Inc., New York, 1987.

[47] P. Julienne and F. Mies. Collisions of ultra-cold trapped atoms. J. Opt. Soc. Am. $B, 6(11): 2257-2269,1989$.

[48] P. S. Julienne. Laser modification of ultracold atomic collisions in optical traps. Phys. Rev. Lett.; 61(6):698-701, 1988.

[49] P. S. Julienne and J. Vigué. Cold collision of ground- and excited-state alkali-metal atoms. Phys. Rev. A, 44(7):4464-4485, 1991.

[50] A. P. Kazantsev, G. I. Surdutovich, and V. P. Yakovlev. Mechanical Action of Light on Atoms. World Scientific, Singapore, 1990.

[51] Wolfgang Ketterle, Kendall B. Davies, Michael A. Joffe, Alex Martin, and David E. Pritchard. High densities of cold atoms in a dark spontaneous-force optical trap. Phys. Rev. Lett., 70(15):2253-2256, 1993.

[52] P. Kohns, P. Buch, W. Süptitz, C. Csambal, and W. Ertmer. On-line measurement of sub-doppler temperatures in a $\mathrm{Rb}$ magneto-optical trap-by-trap centre oscillations. Europhys. Lett., 22(7):517-522, 1993.

[53] W. K. Lai, K. A. Suominen, B. M. Garraway, and S. Stenholm. Dissipation effects on wave packets in level crossings: a comparison between two numerical approaches. Phys. Rev. A, 47(6):4779-4785, 1993.

[54] P. D. Lett, W. D. Phillips, S. L. Rolston, C. E. Tanner, R. N. Watts, and C. I. Westbrook. Optical molasses. J. Opt. Soc. Am. B, 6(11):2084-2107, 1989.

[55] Paul D. Lett, Richard N. Watts, Christoph I. Westbrook, and William D. Phillips. Observation of atoms laser cooled below the Doppler limit. Phys. Rev. Lett., 61(2):169-172, 1988.

[56] K. Lindquist, M. Stephens, and C. Wieman. Experimental and theoretical study of the vapor-cell Zeeman optical trap. Phys. Rev. A, 46(7):4082-4090, 1992.

[57] R. Loudon. The Quantum Theory of Light. Oxford University Press, Oxford, 1983.

[58] L. Marcassa, V. Bagnato, Y. Wang, C. Tsao, J. Wiener, O. Dulieu, Y. Band, and P. Julienne. Collisional loss rate in a magneto-optical trap for sodium atoms: light-intensity dependence. Phys. Rev. A, 47(6):R4563-R4566, 1993. 
[59] H. S. W. Massey. Electronic and Ionic Impact Phenomena. Oxford University Press, London, 1971.

[60] Melles Griot. Optics Guide 5.

[61] B. Mollow. Power spectrum of light scattered by two-level systems. Phys. Rev., 188(5):1969-1975, 1969.

[62] K. Mølmer. Private communication.

[63] K. Mølmer. Friction and diffusion coefficents for cooling of atoms in laser fields with multidimensional periodicity. Phys. Rev. A, 44(9):5820-5832, 1991.

[64] Klaus Mølmer, Yvan Castin, and Jean Dalibard. Monte Carlo wave-function method in quantum optics. J. Opt. Soc. Am. B, 10(3):524-539, 1993.

[65] C. Monroe, E. A. Cornell, C. A. Sackett, C. J. Myatt, and C. E. Wieman. Measurement of Cs-Cs elastic scattering at $T=30 \mu \mathrm{K}$. Phys. Rev. Lett., 70(4):414-417, 1993.

[66] C. Monroe, W. Swann, H. Robinson, and C. Wieman. Very cold trapped atoms in a vapor cell. Phys. Rev. Lett., 65(13):1571-1574, 1990.

[67] M. G. Peters, D. Hoffmann, J. D. Tobiason, and T. Walker. Laser-induced ultracold $\mathrm{Rb}\left(5 S_{1 / 2}\right)+\mathrm{Rb}\left(5 P_{1 / 2}\right)$ collisions. Phys. Rev. A, 50(2):906-909, 1994.

[68] W. Petrich, M. H. Anderson, J. R. Ensher, and E. A. Cornell. Behavior of atoms in a compressed magneto-optical trap. J. Opt. Soc. Am. B, 11(8):1332-1335, 1994.

[69] Wolfgang Petrich, Michael H. Anderson, Jason R. Ensher, and Eric A. Cornell. Stable, tightly confining magnetic trap for evaporative cooling of neutral atoms. Phys. Rev. Lett., 74(17):3352-3355, 1995.

[70] M. Prentiss, A. Cable, J. E. Bjorkholm, Steven Chu, E. L. Raab, and D. E. Pritchard. Atomic density-dependent losses in an optical trap. Opt. Lett., 13(6):452-454, 1988.

[71] E. L. Raab, M. Prentiss, Alex Cable, Steven Chu, and D. Pritchard. Trapping of neutral sodium atoms with radiation pressure. Phys. Rev. Lett., 59(23):2631-2634, 1987.

[72] R. J. Rafac, C. E. Tanner, A. E. Livingston, K. W. Kukla, H. G. Berry, and C. A. Kurtz. Precision lifetime measurments of the $6 p^{2} P_{1 / 2,3 / 2}$ states in atomic cesium. Phys. Rev. A, 50(3):R1976-R1979, 1994.

[73] C. Salomon, J. Dalibard, W. D. Phillips, A. Clairon, and S. Guellati. Laser cooling of cesium atoms below $3 \mu \mathrm{k}$. Europhys. Lett., 12(8):683-688, 1990.

[74] D. Sesko, T. Walker, C. Monroe, A. Gallagher, and C. Wieman. Collisional losses from a light-force atom trap. Phys. Rev. Lett., 69(6):961-964, 1989. 
[75] D. W. Sesko, T. G. Walker, and C. E. Wieman. Behaviour of neutral atoms in a spontaneous force trap. J. Opt. Soc. Am. B, 8(5):946-958, 1991.

[76] A. M. Steane. Laser cooling of atoms. D.Phil. thesis, Oxford University, 1991. Unpublished.

[77] A. M. Steane, M. Chowdhury, and C. J. Foot. Radiation force in the magnetooptical trap. J. Opt. Soc. Am. B, 9(12):2142-2158, 1992.

[78] A. M. Steane and C. J. Foot. Laser cooling below the Doppler limit in a magnetooptical trap. Europhys. Lett., 14(3):231-236, 1991.

[79] A. M. Steane, G. Hillenbrand, and C. J. Foot. Polarization gradient cooling in a one-dimensional $\sigma^{+}-\sigma^{-}$configuration for any atomic transition. J. Phys. B, 25:4721-4743, 1992 .

[80] H. T. C. Stoof. Atomic Bose gas with a negative scattering length. Phys. Rev. A, 49(5):3824-3830, 1994.

[81] K. A. Suominen, M. J. Holland, K. Burnett, and P. S. Julienne. Excited-state survival probabilities for cold collisions in a weak laser field. Phys. Rev. A, 49(5):38973902, 1994.

[82] E. Tiesinga, B. Verhaar, H. Stoof, and D. van Bragt. Spin-exchange frequency shift in a cesium atomic fountain. Phys. Rev. A, 45(5):R2671-R2673, 1992.

[83] C. G. Townsend, C. J. Cooper, N. H. Edwards, C. J. Foot, K. P. Zetie, A. M. Steane, P. Szriftgiser, H. Perrin, and J. Dalibard. Phase-space density in the magneto-optical trap. Phys. Rev. A, 52(2):1423-1440, 1995.

[84] P. Ungar, D. Weiss, E. Riis, and S. Chu. Optical molasses and multilevel atoms: theory. J. Opt. Soc. Am. B, 6(11):2058-2071, 1989.

[85] B. J. Verhaar, K. Gibble, and S. Chu. Cold-collision properties derived from frequency shifts in a cesium fountain. Phys. Rev. A, 48(5):R3429-R3432, 1993.

[86] T. Walker, D. Sesko, and C. Wieman. Collective behavior of optically trapped neutral atoms. Phys. Rev. Lett., 64(4):408-411, 1990.

[87] C. D. Wallace, T. P. Dinneen, K. Y. N. Tan, T. T. Grove, and P. L. Gould. Isotopic difference in trap loss collisions of laser cooled rubidium atoms. Phys. Rev. Lett., 69(6):897-890, 1992.

[88] C. D. Wallace, T. P. Dinneen, K. Y. N. Tan, A. Kumarakrishnan, P. L. Gould, and J. Javanainen. Measurements of temperature and spring constant in a magnetooptical trap. J. Opt. Soc. Am. B, 11(5):703-711, 1994.

[89] H. Wallis, J. Werner, and W. Ertmer. Magneto-optical trapping of atoms. Com. At. Mol. Phys., 28(5):275-300, 1993. 
[90] J. Werner, H. Wallis, G. Hillenbrand, and A. Steane. Laser cooling by $\sigma^{+}-\sigma^{-}$ circularly polarized beams of unequal intensities. J. Phys. B, 26:3063-3080, 1993.

[91] D. Wineland and H. Dehmelt. Proposed $10^{14} \Delta \nu<\nu$ laser fluorescence spectroscopy on $\mathrm{Tl}^{+}$mono-ion oscillator III. Bull. Am. Phys. Soc., 20:637, 1975. 
